\title{
WestVirginiaUniversity
}

THE RESEARCH REPOSITORY @ WVU

Graduate Theses, Dissertations, and Problem Reports

2019

\section{Quantifying Human Biological Age: A Machine Learning Approach}

Syed Ashiqur Rahman

West Virginia University, srahman2@mix.wvu.edu

Follow this and additional works at: https://researchrepository.wvu.edu/etd

Part of the Applied Statistics Commons, Artificial Intelligence and Robotics Commons, Statistical Methodology Commons, Statistical Models Commons, Survival Analysis Commons, and the Theory and Algorithms Commons

\section{Recommended Citation}

Rahman, Syed Ashiqur, "Quantifying Human Biological Age: A Machine Learning Approach" (2019). Graduate Theses, Dissertations, and Problem Reports. 7376.

https://researchrepository.wvu.edu/etd/7376

This Dissertation is protected by copyright and/or related rights. It has been brought to you by the The Research Repository @ WVU with permission from the rights-holder(s). You are free to use this Dissertation in any way that is permitted by the copyright and related rights legislation that applies to your use. For other uses you must obtain permission from the rights-holder(s) directly, unless additional rights are indicated by a Creative Commons license in the record and/ or on the work itself. This Dissertation has been accepted for inclusion in WVU Graduate Theses, Dissertations, and Problem Reports collection by an authorized administrator of The Research Repository @ WVU.

For more information, please contact researchrepository@mail.wvu.edu. 


\title{
Quantifying Human Biological Age : A Machine Learning Approach
}

\author{
Syed Ashiqur Rahman \\ Dissertation submitted to the \\ Benjamin M. Statler College of Engineering and Mineral Resources \\ at West Virginia University \\ in partial fulfillment of the requirements for the degree of \\ Doctor of Philosophy \\ in \\ Computer Science
}
Donald Adjeroh, Ph.D., Chair
Xin Li, Ph.D., Gianfranco Doretto, Ph.D.
Yanfang Ye, Ph.D.
Peter Giacobbi, Ph.D.

Lane Department of Computer Science and Electrical Engineering

\author{
Morgantown, West Virginia \\ 2019
}

Keywords: Aging, Deep Learning, Machine Learing, Biological Age, Obesity, Mortality Modeling BMI, Physical Activity, Body-shape, SBSI, Neighborhood Contexts

(C) 2019 Syed Ashiqur Rahman 


\section{ABSTRACT \\ Quantifying Human Biological Age : A Machine Learning Approach Syed Ashiqur Rahman}

Quantifying human biological age is an important and difficult challenge. Different biomarkers and numerous approaches have been studied for biological age prediction, each with its advantages and limitations. In this work, we first introduce a new anthropometric measure (called Surface-based Body Shape Index, SBSI) that accounts for both body shape and body size, and evaluate its performance as a predictor of all-cause mortality. We analyzed data from the National Health and Human Nutrition Examination Survey (NHANES). Based on the analysis, we introduce a new body shape index constructed from four important anthropometric determinants of body shape and body size: body surface area (BSA), vertical trunk circumference (VTC), height (H) and waist circumference (WC). SBSI is generally linear with age, and increases with increasing mortality, when compared with other popular anthropometric indices of body shape. We then investigate whether human body shape can be exploited for reliable age estimation for adult humans. We introduce a new multi-stage approach, based on human body measurements. Specifically, we develop an eigen body shape model, and use this to perform body shape clustering. Each cluster contains individuals with similar body shapes as captured by the eigen body shape model. First, we perform initial age estimation based on the body shape model. This initial estimate is then used to assign the subject into a probable age group. The second stage of estimation is then performed by using a specific estimation model as determined by the age group and body shape model. We then apply information from the neighborhood context to further improve estimation accuracy and stability. Experimental results show that, with appropriate modeling, human body shape can be used in human age estimation. We obtain a mean absolute error (MAE) of 5.90 years on the NHANES dataset, using 10-fold cross-validation. We then study the question of whether blood biomarkers can be used for reliable biological age estimation. We propose a new biological age estimation method, and investigate the performance of the new method against popular biological age estimation methods. We introduce a centroid based approach, using the notion of age neighborhoods. Specifically, we develop a model, based on which we compute biological age using blood biomarkers. Compared with current popular methods for biological age prediction, our results show that, the proposed age neighborhood model is robust, and results in improved performance in human biological age prediction. Furthermore, we investigate whether human locomotor activity can be exploited for biological age estimation for adult humans. We introduce an approach based on deep convolutional long short term memory (ConvLSTM) to predict biological age, using human physical activity as recorded by a wearable device. We consider five deep biological age estimation models including the proposed approach and compare their performance on the NHANES physical activity dataset. Results on mortality hazard prediction using both the Cox proportionality hazard model and Kaplan-Meier curves each show that the proposed method for estimating biological age outperforms other state-of-the-art approaches. This work has significant implications in various fields, such as health assessment, forensic science, biometrics, security, and in vaccination and immunization when the true age of the subject is unknown. Our work also has implications in combining wearable sensors and deep learning techniques for improved health monitoring, for instance, in a mobile health environment. 
To my wonderful parents, sister, and wife 


\section{Acknowledgements}

My graduate journey at West Virginia University has been wonderful due to kind support and encouragement provided towards me by many different people. It is my pleasure to thank them.

I would like to express deepest gratitude to my supervisor, Dr. Donald A. Adjeroh without his relentless support this dissertation would not be possible. I am grateful for the seed of excitement and passion for research he sowed in me. I thank him for his guidance and support and I appreciate his knowledge and wit. Whenever I was stuck with an obstacle, he was available to help me out. I found him very caring, generous, and patient with me which made my graduate school experience a pleasant one. He has been a source of inspiration to me and will remain so. I will forever cherish that I had the opportunity to work with him.

I thank my dissertation committee members, specially Prof. Xin Li, I took three courses under him, he is a wonderful teacher and I learned a lot. I audited Machine Learning course under Dr. Gianfranco Doretto, and very grateful to him. Under Dr. Yanfang Ye, I had two major courses, and in one of our course final project work we published a conference paper. She always encouraged me about my research. I thank Dr. Peter Giacobbi for agreeing to be in my committee, I believe his inputs are very helpful for this dissertation.

Besides those who contributed to my dissertation, I acknowledge the people who influenced my life within and outside graduate school. I am indebted to my lab-mates for their encouragements. I have been fortunate enough to have excellent friends over the years who have inspired me for pursuing my graduate study. There are too many to list here, but few deserve special mention: Dr. Mahmood Hossain, Nausheen Hossain, Dr. Mahfuzur Rahman, Tania Sultana, Jahangir Alam, Mamun Rashid, Dr. Prokash Paul, Dr. Osman Gani, Dr. Daud Isa, Dr. Rakibul Khan, Musfique Ahmed, Rifat Anwar, S M Ashikur Rahman, Umma Halima Jolly, and my best friend Dr. Tozammel Hossain.

I thank my parents and my sister Mahbuba Rahman for their patience and continuous support. They were always confident in my abilities and encouraged me to pursue a $\mathrm{PhD}$ in the United States of America. Finally, my wife Tazin Afrin is an amazing individual whose love, patience and sacrifices kept me on the track during trying times. It would be impossible without her support to complete this dissertation. 


\section{Contents}

\begin{tabular}{|ll}
\hline Acknowledgements & iv
\end{tabular}

\begin{tabular}{|l|l}
\hline List of Figures & ix
\end{tabular}

List of Tables $\quad$ xi

\begin{tabular}{lll}
\hline 1 & Introduction & 1
\end{tabular}

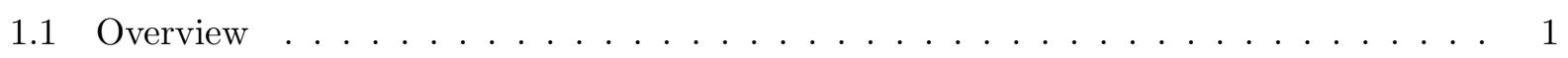

1.2 The Problem . . . . . . . . . . . . . . . . . . . . . . . . 2

$1.2 .1 \quad$ Obesity and Health Risk . . . . . . . . . . . . . . . . . . . . . . . 2

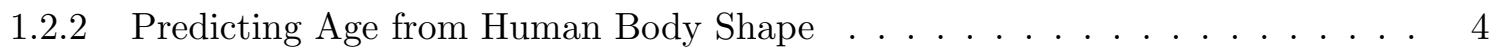

1.2.3 Quantifying Biological Age Using Biomarkers . . . . . . . . . . . . . . . . . 5

1.2.4 Quantifying Biological Age Using Physical Activity . . . . . . . . . . . . . 6

1.3 Thesis Contribution $\ldots \ldots \ldots \ldots \ldots \ldots$

1.3 .1 Obesity and Health Risk. . . . . . . . . . . . . . . . . . 6

1.3.2 Chronological Age from Body Shape. . . . . . . . . . . . . . . . . . 7

1.3 .3 Biological Age via Age Neighborhoods. . . . . . . . . . . . . . . . . 8

1.3.4 Deep Learning for Biological Age Analysis Using Locomotor Physical Activity 8

1.3 .5 Publications from $\mathrm{PhD}$ Work $\ldots \ldots \ldots \ldots$. . . . . . . . . . . . 9

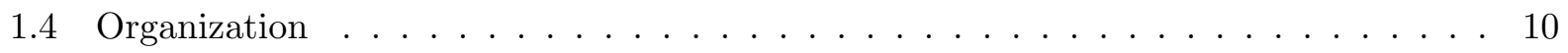

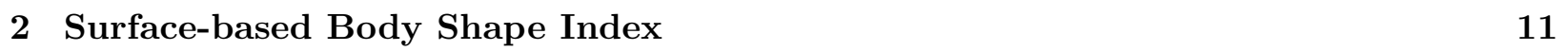

2.1 Materials and Methods . . . . . . . . . . . . . . . . . . . . . . . 13

2.1 .1 Datasets. . . . . . . . . . . . . . . . . . . 13 
$2.1 .2 \quad$ Study Variables. . . . . . . . . . . . . . . . . . . . . . . . . . 14

$2.1 .3 \quad$ Surface-based Body Shape Index (SBSI) . . . . . . . . . . . . . . . 15

$2.1 .4 \quad$ SBSI Construction. . . . . . . . . . . . . . . . . . . . 18

2.1 .5 Statistical Analysis. . . . . . . . . . . . . . . . . . . . . . 19

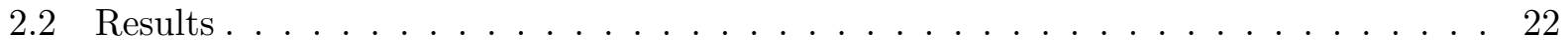

$2.2 .1 \quad$ Higher SBSI with Increasing Age. . . . . . . . . . . . . . . . . 22

2.2 .2 Higher Mortality Hazard for Increasing SBSI. . . . . . . . . . . . . . . . 23

$2.2 .3 \quad$ Improved Modeling for All-Cause Mortality using SBSI. . . . . . . . . . . . . 24

2.2 .4 Mortality Hazard using SBSI on BMI Categories. . . . . . . . . . . . . . 26

2.3 Discussion . . . . . . . . . . . . . . . . . . . . . . . . . 30

2.4 Limitations of the approach. . . . . . . . . . . . . . . . . . 34

\begin{tabular}{|lll|}
\hline 3 & Estimating Adult Age from Human Body Shape & 37
\end{tabular}

3.1 Introduction . . . . . . . . . . . . . . . . . . . . . . . 37

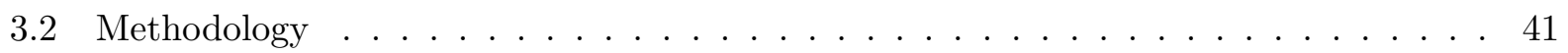

3.2 .1 Dataset . . . . . . . . . . . . . . . . . . . . . . 4 41

$3.2 .2 \quad$ Data Availability $\ldots \ldots \ldots \ldots \ldots \ldots \ldots$. . . . . . . . . . . . . . . 42

3.2 .3 Key Observations and Motivation $\ldots \ldots \ldots \ldots$. . . . . . . . . 42

3.2 .4 Simple Prediction $\ldots \ldots \ldots \ldots$

3.2 .5 Multi-stage Age Estimation $\ldots \ldots \ldots \ldots \ldots$. . . . . . . . . 47

3.2 .6 Incorporating Neighborhood Contexts . . . . . . . . . . . . . . . . . . . . 49

3.3 Results . . . . . . . . . . . . . . . . . . . . . . . . . 53

$3.3 .1 \quad$ Baseline Results $\ldots \ldots \ldots \ldots \ldots \ldots$. . . . . . . . . . . . . . . 53

3.3.2 Eigen Body Shape Clustering Improves Age Estimation Accuracy . . . . . . . 55

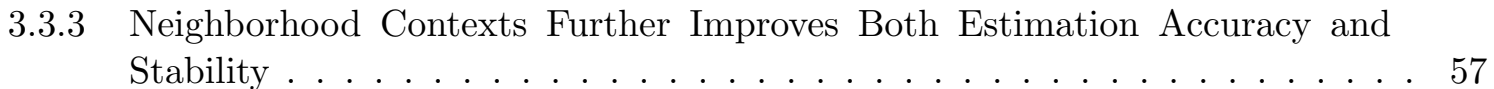

3.3 .4 Impact of Gender. . . . . . . . . . . . . . . . . . . . . 59

3.4 Discussion . . . . . . . . . . . . . . . . . . . . . . . . . . . . 61

3.4 .1 Cross Database Evaluation $\ldots \ldots \ldots \ldots \ldots$. . . . . . . . . . . 61

3.4 .2 Comparative Results $\ldots \ldots \ldots \ldots \ldots \ldots$ 
3.4 .3 Impact of Age Grouping and Feature Interaction . . . . . . . . . . . . . . 64

4 Centroid of Age Neighborhoods for Biological Age Estimation 66

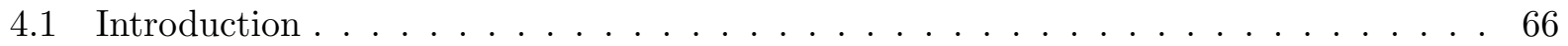

4.2 Methodology . . . . . . . . . . . . . . . . . . . . 69

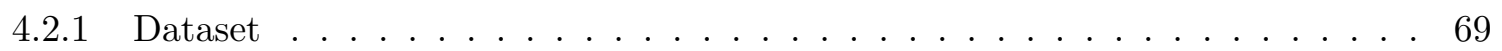

$4.2 .2 \quad$ Characteristics of the Dataset or Sample . . . . . . . . . . . . . . . . . 69

$4.2 .3 \quad$ Symbols/Notations Used . . . . . . . . . . . . . . . . . 71

$4.2 .4 \quad$ Centroid BA: New Approach to Estimating BA $\ldots \ldots \ldots \ldots \ldots$

4.2 .5 Medoid BA: Estimating BA using Medoid of Age Neighborhoods . . . . . . . 75

4.3 Results . . . . . . . . . . . . . . . . . . . . . . . . . . . . . . . . 77

$4.3 .1 \quad$ Cox PH model. . . . . . . . . . . . . . . . . . . . . . . 77

$4.3 .2 \quad$ KM Curves and Log-rank test . . . . . . . . . . . . . . . . . . . 78

4.3 .3 ROC of AUC $\ldots \ldots \ldots \ldots \ldots \ldots$

4.3 .4 Results for Approach $2 \ldots \ldots \ldots \ldots \ldots$. . . . . . . . . . . . . . . . .

4.3 .5 Comparative Results . . . . . . . . . . . . . . . . . . . . 84

4.4 Discussion . . . . . . . . . . . . . . . . . . . . . . . . . . 85

$4.4 .1 \quad$ Impact of Number of Neighbors. $\ldots \ldots \ldots \ldots$. . . . . . . . . 85

4.4 .2 Impact of Gender. . . . . . . . . . . . . . . . . . . . . . 87

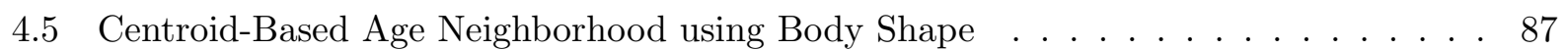

$4.5 .1 \quad$ KM Curves and Log-rank test . . . . . . . . . . . . . . . . . . . . . . . 88

4.5 .2 Human Anthropometry can predict BA $\ldots \ldots \ldots \ldots$. . . . . . . . 91

$4.5 .3 \quad$ BA Estimation using Combined Features $\ldots \ldots \ldots$. . . . . . . . . . . . . . . 91

5 Deep Learning for Analyzing Biological Age Using Physical Activity 94

5.1 Introduction . . . . . . . . . . . . . . . . . . . . 94

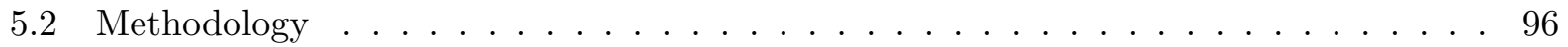

5.2 .1 Datasets . . . . . . . . . . . . . . . . . . 96

$5.2 .2 \quad$ Data Transformation \& Data Representation . . . . . . . . . . . . . . . 97

$5.2 .3 \quad$ LSTM estimation of Biological Age . . . . . . . . . . . . . . . . . . . . . 98 
$5.2 .4 \quad$ Statistical Analysis $\ldots \ldots \ldots$. . . . . . . . . . . . . . . . 102

5.3 Results . . . . . . . . . . . . . . . . . . . . . . . 103

5.3 .1 Human Locomotor Activity is associated with chronological age. . . . . . . . 103

5.3.2 ConvLSTM* estimated BA acceleration using physical activity data is associated with BA from biomarkers . . . . . . . . . . . . . . . . . . 104

5.3.3 Deep learning biological age using ConvLSTM* ${ }^{*}$ on physical activity data leads to improved modeling of all-cause mortality. . . . . . . . . . . . . 108

5.4 Discussion . . . . . . . . . . . . . . . . . . . . . . 110

5.4 .1 Parameter Choices . . . . . . . . . . . . . . . . . . . . . . 112

5.4 .2 Impact of Gender $\ldots \ldots \ldots \ldots \ldots \ldots$

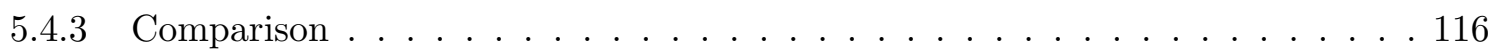

5.4 .4 Limitation . . . . . . . . . . . . . . . . . . . . 118

6 Biological Age from 3D body shape $\quad 119$

6.1 BA Modeling and Estimation using 3D Body Shape . . . . . . . . . . . . . . . 119

6.2 Methodology $\ldots \ldots \ldots \ldots \ldots \ldots$

6.2 .1 Dataset . . . . . . . . . . . . . . . . . . . 120

6.2 .2 NHANES to MakeHuman . . . . . . . . . . . . . . . . . . . . . 121

6.2 .3 Multi-Input Deep Learning Models . . . . . . . . . . . . . . . . . . . 124

6.3 Results . . . . . . . . . . . . . . . . . . . . . . . . . . . 125

6.3 .1 CA Performance . . . . . . . . . . . . . . . . . . 125

6.3.2 Cox Proportional Hazard Model and Kaplan Meier Estimation . . . . . . . . 126

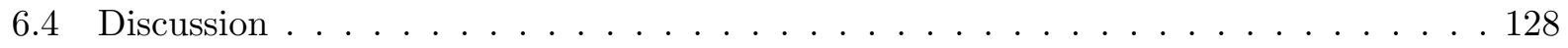

\begin{tabular}{llr}
\hline 7 & Future Work & 129
\end{tabular}

7.1 Geometric deep learning . . . . . . . . . . . . . . . . . . . . . 129

7.2 Aging Biomarkers Discovery using Deep Learning . . . . . . . . . . . . . . . . . . . 129

7.3 Biological Age from DNA methylation . . . . . . . . . . . . . . . . . 130

7.4 Cardiorespiratory Fitness $\ldots \ldots \ldots$. . . . . . . . . . . . . . . . . . . . .

7.5 Variation of BA with ethnic group $\ldots \ldots \ldots \ldots$. . . . . . . . . 130

\begin{tabular}{ll}
\hline Bibliography & 133
\end{tabular} 


\section{List of Figures}

2.1 Relationship between BSA, VTC, height and WC for given BMI categories. The BSA and height, and VTC and height can predict the BMI categories (a, b). BSA and WC (and VTC and WC) show a non-linear relationship for a given BMI category

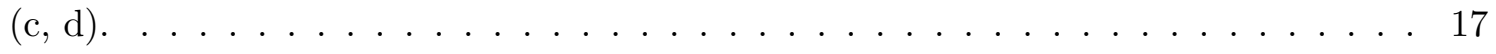

$2.2 \quad$ Variation of different body shape indices with age (in years). . . . . . . . . . . . 23

2.3 Variation of relative death rate with increasing values of SBSI z-score. (a) Female;

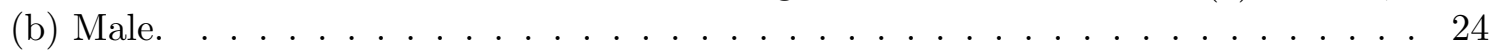

2.4 The Kaplan Meier curves for four body shape indices using all subjects. The SBSI shows a better prediction performance than other body shape measures (with more separation between the curves, and less crossovers). 1st Q, 2nd Q, etc. denote respectively 1st quartile, 2 nd quartile, etc. . . . . . . . . . . . . . . . . 26

2.5 The KM curves using ABSI and SBSI on subjects in the BMI category overweight. a: ABSI (male); b: ABSI (female); c: SBSI (male); d: SBSI (female). As expected, both measures indicate that female subjects have better survival rates when compared with male subjects. SBSI shows an overall better prediction performance than ABSI. 1st Q, 2nd Q, etc. denote respectively 1st quartile, 2nd quartile, etc. . . . . . . . . . 30

2.6 The KM curves using ABSI and SBSI on subjects in the BMI category obese I. a: ABSI (male); b: ABSI (female); c: SBSI (male); d: SBSI (female). . . . . . . . . . . 31

3.1 Variation of different body shape indices with age. $\ldots \ldots \ldots \ldots$

3.2 Proposed framework for multi-stage age estimation using human body measurements. 47

3.3 Impact of body shape clustering on age estimation performance: (a) effect of the number of shape clusters $k$; (b) performance variation with shape clusters $\left(C_{1}, C_{2}, C_{3}, C_{4}\right)$ and age groups $\left(G_{1}: \leq 25, G_{2}: 26-35, G_{3}: 36-45, G_{4}: 46-55, G_{5}: 56-70, G_{6}: 70+\right)$ without neighborhood-based refinement; (c) performance variation with neighborhoodbased refinement; (d) density of estimation error for the final estimated age using gender-specific models (male, female) and non-gender specific model (labeled "all data"). Reported results are based on all test subjects using 10-fold cross validation. 56

$3.4 \quad$ Age group distribution for different body shape clusters. . . . . . . . . . . . . . . . 57 
4.1 Variation of biomarkers with age. Features plotted for average measurements for

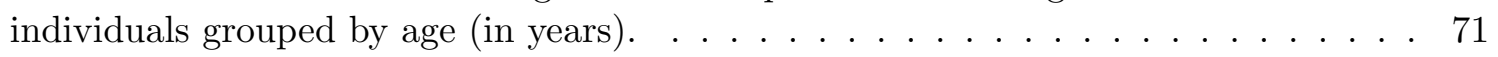

4.2 Proposed framework for centroid based biological age prediction. . . . . . . . . . . 72

4.3 The Kaplan Meier curves for three BA estimation algorithms using biomarker features. Results are reported for (a, c, e) chronological age, and (b, d, f) predicted BA. Q1, Q2, etc. denote 1st quartile, 2nd quartile, etc. . . . . . . . . . . . . 80

4.4 The Kaplan Meier curves for Medoid using biomarker features (a) chronological age, and (b) predicted BA. Q1, Q2, etc. denote 1st quartile, 2nd quartile, etc. . . . . . . 81

4.5 ROC curves for MLR, KD, DNN, Centroid, and Medoid algorithms using biomarker features. Results are reported for applying $\Delta=C A-B A$ for the respective estima-

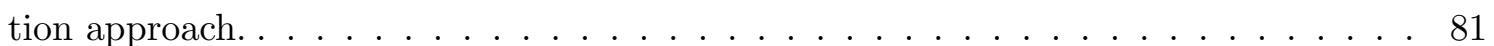

4.6 Impact of parameter $\alpha$ and $\tau$ in Approach2 (a) using Centroid BA, and (b) using Medoid BA. . . . . . . . . . . . . . . . . . . 83

4.7 ROC curves for 4 biological age estimation algorithms using a)Female, and b)Male models separately on biomarker features. . . . . . . . . . . . . . . 86

4.8 Variation of different anthropometric and biomarkers attributes with age. Features plotted for average measurements for individuals grouped by age (in years). . . . . . 88

4.9 The Kaplan Meier curves for using proposed centroid-based biological age (a, c, e), and chronological age $(\mathrm{b}, \mathrm{d}, \mathrm{f})$. Results are reported for applying $\Delta=C A-B A$ on biomarkers, anthropometric features, and combined features. Q1, Q2, etc. denote 1st quartile, 2nd quartile, etc. . . . . . . . . . . . . . . . . 90

4.10 Pairwise uncorrelated attributes which are correlated with age. . . . . . . . . . . . 92

5.1 (a) Basic LSTM cell. П denotes multiplication, while + denotes addition, $\sigma$ is sigmoid function, tanh calculates hyperbolic tangent, (b) Transformation and inner structure of ConvLSTM* . . . . . . . . . . . . . . . . . . 100

$5.2 \quad$ Architecture of proposed ConvLSTM* deep learning method for biological age estimation using human locomotor activity data . . . . . . . . . . . . . . . . 101

5.3 Variation of average physical activity with age. Plotted for individuals grouped by

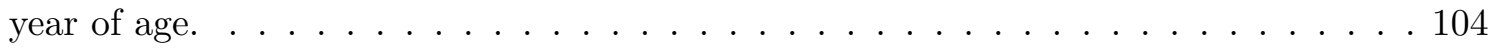

5.4 Variation of two biomarkers (albumin, and hemoglobin) with age. Features plotted in average measurements for individuals grouped by year of age. . . . . . . . . 106

5.5 Similarity between average biological estimated age using biomarkers using KD method and physical activity using ConvLSTM* over the age range 18-84. (a) Estimated biological ages, (b) density of estimated biological ages (These results are for common individuals in both the biomarker and physical activity datasets). . . . . . . 107 
5.6 The Kaplan Meier curves for estimated biological ages (BA) based on the physical activity applying $\eta=\frac{C A-B A}{C A}$ for both chronological and estimated biological ages. (a, c, e) refer to estimated biological ages, and (b, d, f) chronological age respectively. Q1, Q2, Q3, and Q4 denote 1st, 2nd, 3rd, and 4th quartiles, respectively. The Eta prefix in the plot legend is followed by the method used to estimate BA and calculate

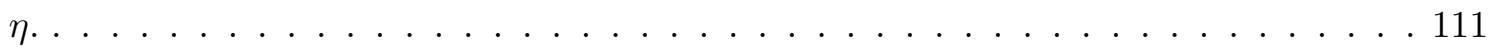

5.7 The Kaplan Meier curves for applying $\eta=\frac{C A-B A}{C A}$ on the physical activity (a) female, and (b) male. Q1, Q2, Q3, and Q4 denote 1st, 2nd, 3rd, and 4th quartiles, respectively. The Eta prefix in the plot legend is followed by the method used to estimate BA and calculate $\eta \ldots \ldots \ldots \ldots \ldots \ldots$

6.1 Generated 3D meshes from sample subjects of NHANES for each BMI categories (a) female subjects Underweight (18.37), Normal (19.95), Overweight (29.73), Obese I (32.97), Obese II (38.57), Obese III (43.42) (from left to right respectively), (b) male subjects Underweight (16.96), Normal (23.65), Overweight (28.84), Obese I (32.49), Obese II (37.08), Obese III (45.34) (from left to right respectively). . . . . . . . . . 120

6.2 Generated 2D images from NHANES from different viewing angles for a female subject (a)front, (b) back, (c) left, (d) right, (e) top, and (f) bottom. . . . . . . . . . 122

6.3 Generated 2D images from NHANES from different viewing angles for a male subject (a)front, (b) back, (c) left, (d) right, (e) top, and (f) bottom. . . . . . . . . . . . 123

6.4 Variations of multi-input model to estimate biological age. . . . . . . . . . . . . 124

6.5 The Kaplan Meier curves for estimated biological ages (BA) based on the physical activity applying $\eta=\frac{C A-B A}{C A}$ for (a) ConvLSTM on the physical activity, (b) 2D CNN on the Makehuman images (front), (c) function architecture of 2D CNN, and ConvLSTM on the combined features (front), (d) 2D CNN on the Makehuman images (3 images), (e) function architecture of 2D CNN, and ConvLSTM on the combined features (3 images). Q1, Q2, Q3, and Q4 denote 1st, 2nd, 3rd, and 4th quartiles,

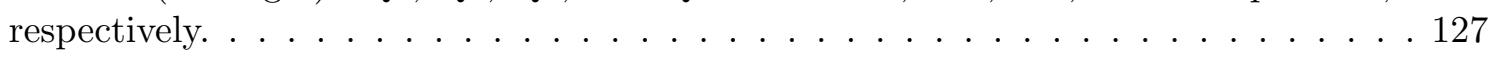




\section{List of Tables}

2.1 Key anthropometric attributes for study participants. . . . . . . . . . . 20

2.2 Correlation coefficient between anthropometric measures. . . . . . . . . . . . . 20

2.3 Summary of mortality hazard ratio for four key anthropometric measures. . . . . . 25

2.4 Summary of $\chi^{2}$-distances for KM survival curves for four key anthropometric measures. 25

2.5 Results of Cox proportionality hazard modeling using different anthropometric mea-

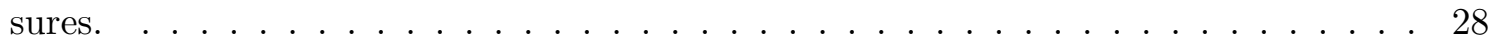

2.6 Results using the log-rank test on the KM curves for different anthropometric measures. 29

2.7 Key anthropometric attributes for study participants (using the CAESAR dataset). 34

2.8 Results for Cox Proportional Hazard applied to different age categories. . . . . . . . 35

2.9 Log-rank test results for different age category. $\ldots \ldots \ldots \ldots$

3.1 Anthropometric attributes for study participants using the NHANES dataset. . . . . 43

3.2 Correlation coefficient between anthropometric measurements: Pearson's $\rho$ (upper half), and Kendall's $\tau$ (lower half). . . . . . . . . . . . . . . . . . 44

3.3 Simple Age Prediction. Results are shown for all test subjects, using three classifi-

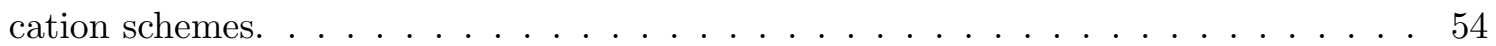

3.4 Improving estimation accuracy and stability using neighborhood contexts. Results (mean absolute error (MAE)) are shown for all subjects, using 10-fold cross validation, where $I D_{1}$ corresponds to using $\left(\alpha_{1}, \alpha_{2}, \alpha_{3}\right)=(0,0.5,0.5), I D_{25}(0.5,0.25$, $0.25), I D_{35}(1,0,0)$. Results are grouped by the probable age groups (based on first level of age estimation). $\ldots \ldots \ldots \ldots \ldots \ldots$

3.5 Weights for adaptive neighborhood-based refinement. . . . . . . . . . . . . 60

3.6 Summary results showing the impact of gender on the performance of the proposed neighborhood-based refinement for age estimation. Results (mean absolute error (MAE)) are shown for female-only and male-only subjects, using 10-fold cross validation, where $I D_{35}$ corresponds to using $\left(\alpha_{1}, \alpha_{2}, \alpha_{3}\right)=(1,0,0) . \ldots \ldots \ldots$ 
3.7 Comparative results of Age Prediction. . . . . . . . . . . . . . . . . 63

4.1 Key biomarker attributes for study participants of NHANES dataset. . . . . . . . . . 70

4.2 Correlation coefficient (Pearson's $\rho$ ) between biomarkers and between biomarkers and age. Pearson $\rho$ (upper half), and Kendall $\tau$ (lower half). . . . . . . . . . . 70

4.3 Cox PH results for biological age estimation methods. . . . . . . . . . . . . . 78

4.4 Log Rank results $\left(\chi^{2}\right.$-distance) for mortality modeling using four biological age prediction methods applied to the blood biomarkers. . . . . . . . . . . . 79

4.5 Area under the curve (AUC) of receiver operating characteristics (ROC) curves. . . 79

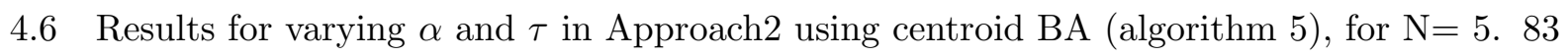

\begin{tabular}{|lll|l|}
\hline 4.7 & Results for varying $\alpha$ and $\tau$ in Approach2 using medoid BA (algorithm & 6 ), for $\mathrm{N}=5$. & 84
\end{tabular}

4.8 Overall comparative results on biological age prediction. . . . . . . . . . . . 84

4.9 Impact of number of neighbors on mean absolute error (MAE), log-rank tests on estimated biological age, delta (CA-BA). . . . . . . . . . . 86

4.10 Area under curve (AUC) of receiver operating characteristics (ROC) curves for separate male, female models. $\ldots \ldots \ldots \ldots \ldots$. . . . . . . . . . . . . .

4.11 Cox PH results for biological age estimation methods. . . . . . . . . . . . . 89

4.12 Log Rank results for biological age estimation methods. . . . . . . . . . . . . . . 89

$5.1 \quad$ Key attributes for study participants using the NHANES (2003-2006) dataset. . . . 102

5.2 Correlation between average physical activity, chronological age and the biomarkers. 106

5.3 Results of the Cox proportionality hazard (Cox PH) models applied on the normalized biological age acceleration $\eta=(C A-B A) / C A$ for estimated biological ages. . . 108

5.4 Results of the log-rank test applied on the normalized biological age acceleration $\eta=(C A-B A) / C A$ using the estimated biological ages. . . . . . . . . . . . 110

5.5 Results of the Cox Proportionality Hazard model (CoxPH) applied on the normalized biological age acceleration $\eta=(C A-B A) / C A$ for separate female, male subjects. .113

5.6 Results of Log-rank tests applied on the normalized biological age acceleration $\eta=$ $(C A-B A) / C A$ for separate female, male subjects. $\ldots \ldots \ldots$. . . . . . . 114

5.7 Log rank results applying $\left(\eta=\frac{C A-B A}{C A}\right)$ for different SBSI categories. Results are shown for model with all subjects, female model and male models separately. Q1, Q2, etc. denote 1st quartile, 2nd quartile, etc. . . . . . . . . . . . . 115

5.8 Log rank results applying normalized biological age acceleration $\left(\eta=\frac{C A-B A}{C A}\right)$ for different WHtR quartiles. Results are shown for model with all subjects, female model and male models separately. Q1, Q2, etc. denote 1st quartile, 2nd quartile, etc.116 
5.9 Results of the Deep learning Age Prediction methods. . . . . . . . . . . . . . 117

6.1 Results of the deep neural network age prediction methods. . . . . . . . . . . . 125

6.2 Results of the Cox proportional hazard (Cox PH) model and Log-rank test applied on the normalized biological age acceleration $\eta=(C A-B A) / C A$ for estimated

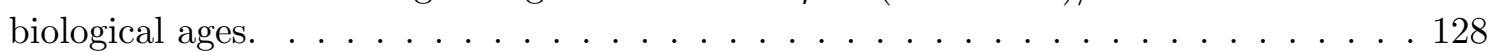

7.1 Ethnic variation results of the Cox proportional hazard (Cox PH) model and Log-

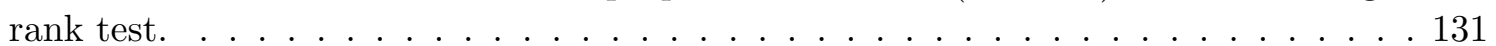

S1 Variations of window size for computing the moving averages after log-transformation.132

S2 Choosing $\lambda$ parameters for the Box-Cox transformation. . . . . . . . . . . . . 132 


\section{Chapter 1}

\section{Introduction}

\subsection{Overview}

Age estimation is an important problem that has witnessed an increased attention, given its role in various daily activities, from health assessment [94], to social interaction [103], to forensic science [64, 145], to security and identity profiling [57]. Although age estimation has been practiced for centuries, accurate age estimation is known to be a very difficult problem. Doing this automatically by a machine is an even much more onerous task [57,65]. The major

challenge is that most measures used to characterize age, for instance, visual appearance, and biological/physiological markers vary significantly from person to person, even for people with the same chronological age. The reason is that many unknowns (e.g., genetics 48, 145], nutrition [125], body shape [118, , health condition [125], social conditions [71], life style [36], weather [13], and even cultural considerations [13]), all contribute to influence the perceived age of an individual. There are various scenarios or applications when the chronological age is required, but the true birth date is unknown, and a genuine birth certificate may not be available. Examples here include in forensic and crime investigations 64, 121, 145, biometrics [53, 56, 65] for border control and security, competitive sports and age-based competitions, and in immunization and vaccination, even for adults [7], for instance, in a refugee camp. 
Age has a deep connection with health and mortality 21,89,97]. Aging is a gradual process that results in increased health risk, and mortality over time. In general, a younger person is expected to have a better health condition and his/her mortality hazard should be low in comparison with a relatively older person. But two different people of the same age may have very different health conditions and mortality hazards. This brings up the debate on "chronological" versus "biological" age.

Chronological age is typically what we know and is based on the date of birth. However, biological age is based on the interesting, yet confounded, idea that a person's true age can be different from his/her chronological age. Biological age (sometimes called functional age [129]) lacks a precise definition, but it is often viewed as the true age of an individual in the gerontology and aging research community [83]. The common idea is that, biological age provides a better estimator of the true life expectancy of the individual than his or her chronological age. Quantification of biological age is a difficult challenge, since there is no well defined criteria.

\subsection{The Problem}

\subsubsection{Obesity and Health Risk}

Obesity, with its dual complications of diabetes mellitus and cardiovascular disease (CVD), has emerged as a major public health challenge [50,98, 105]. Obesity is identified by the World Health Organization (WHO) as a global epidemic [141]. In the US, obesity prevalence remains high at $35.7 \%$ of the adult population [104, while $68 \%$ are classified as obese or overweight [50], with the highest rates being found among the populations that are poor, have lower education, and are minority groups [50]. The picture for childhood and adolescent obesity is no better, with $16.9 \%$ obesity prevalence, and $31.8 \%$ classified as obese or overweight [104, and thus at the risk of developing insulin resistance, dyslipidemia, or hypertension at an early age [117. This trend is mirrored by the high incidence of dia- 
betes, which has shown a similarly high prevalence rates [5]. The problem of obesity is attributed to the issue of imbalance between energy intake and energy expenditure in the body [116]. The problem is directly connected to the quantity of adipose depots (body fat). Adiposity is associated with increased risk of many chronic diseases in the general population 27, 34, 37, 47, 81. Obesity is known to be associated with diabetes, and various forms of cardiovascular disease (CVD). Other associated complications include depression, mobility issues, some forms of cancer [55], sleep apnea [107], osteoarthritis, among others.

Many different anthropometric measures have been used to assess adiposity. The body mass index (BMI) is one of the best known indices of relative adiposity or excess body weight in the association of body composition with mortality. Individuals are often grouped into BMI categories 52,141 from underweight to obese III. Risk of CVD and diabetes tends to increase with increasing BMI. The association of BMI with mortality in the general population is usually found to exhibit a U-shaped [15,51] or J-shaped [90, 95] curve. Using BMI-defined categories Flegal et al [51] showed, that obese and underweight individuals had a higher death rate, while normal weight and overweight individuals had a relatively similar mortality risk. Some have hypothesized that the non-linear relationship observed between BMI and mortality may be a consequence of BMI being a composite of both fat and fat-free mass 14,22, not simply a surrogate for overall adiposity. These observations point to the core limitation of BMI as a measure of adiposity. Several studies have shown that adjustment for waist circumference, a surrogate for abdominal adiposity 66, 75, 131, eliminates or attenuates BMI's nonlinear relationship with mortality 23, 79]. The ABSI (A Body Shape Index) which places more emphasis on waist circumference was proposed by Krakauer and Krakauer [85] as an alternative to the BMI, resulting in a better prediction of mortality hazard. Various other one dimensional (1D) anthropometric measures (e.g., waist circumference, hip circumference, skin folds), and their relation to obesity have also been studied. Example, the waist-to-hip ratio is a better indicator of ischemic heart disease mortality [99], while the waist-to-height ratio (WHtR) provides a better predictor for death, 
heart attack and stroke [126]. Thus there is still need to investigate novel and easy to use methods for rapid profiling of health status and for modeling mortality for a given population.

\subsubsection{Predicting Age from Human Body Shape}

The human body grows and matures with age, especially in children and adolescents. Therefore, the basic approach to age prediction is to compare measurements of physical maturity of the body with age. However, due to individual variations in the timing of skeletal and dental development, the results of any method will be subject to uncertainty when applied to a single individual. Moreover, age prediction is difficult because people in different subgroups age at different rates. The study of aging differences across subgroups necessarily involves the characteristics of people along with their chronological age. Bones are known to mature at fairly standard rates, while growing through childhood [86]. Like normal human growth, bones stop growing around ages 17 to 25 . A young person who is still growing have special growth plates in his/her bones, but at about age 20, the bones stop growing [100]. Clinical growth charts showing the expected growth at a given age are available for both boys and girls (http://www.cdc.gov/growthcharts/clinical_charts.htm). These charts are easily found in pediatric clinics and hospitals, and are often used in routine growth monitoring, and in assessment of healthy body development in children and adolescents. For a child having a normal growth, the charts suggest that we can predict his/her age using height and weight. A popular approach for estimating the age from todlers to adolescent is the use of dental features [142], e.g., by checking the types of teeth visible above the gum line. Age for children and adolescents can also be estimated via microscopic examination of the enamel [147], or by the use of dental radiographs [93]. For adults however, the accuracy of these methods is significantly reduced [121]. Given their dependence on growth, most of these methods used to predict chronological age in children and adolescents do not work for adults. For instance, adult height remains fairly constant until around the seventies, when a decrease with age may be observed. Not surprisingly, various other approaches have been 
proposed for age estimation for adults, from semi-automated to fully automated methods. The methods range from image-based techniques [38, 53, 57, 65] to those that use biomarkers 114, 127, to those that exploit genetic information [67, 137. Age estimation from face is perhaps the most popular [65] of the image-based approaches. We note that while other methods such as body measurements are relatively successful in predicting chronological age for children and adolescents, these age categories pose the most difficult challenge for facebased methods [119]. Thus we identified that age estimation is still an important challenge and more extensive studies require for modeling aging in terms of human body parameters.

\subsubsection{Quantifying Biological Age Using Biomarkers}

To estimate biological age, some age-dependent variables are used [35, 74, 127], and chronological age may or may not be a required attribute/variable depending on the application. The primary approach for BA estimation involves the use of blood biomarkers, such as: C-reactive protein, glycated hemoglobin, albumin, total cholesterol, urea nitrogen, alkaline phosphatase, systolic blood pressure, diastolic blood pressure, pulse, high density lipoprotein, hemoglobin, lymphocyte percent, while blood cell count, hematocrit, red blood cell count, platelet count.

Klemera and Doubal's approach [83] is the most popular, and perhaps, the most effective biological age estimation method $[21,89,97]$. The biological age (BA) estimates are derived based on minimizing the distance between biomarker points and regression lines, and the estimation is performed in a dimensional space of blood biomarkers. Other approaches include multiple linear regression (MLR) [89, principal component analysis (PCA) 87], and combination of MLR with PCA features [89]. Levine [89] compared the performance of five BA algorithms in terms of their ability to predict mortality. Klemera and Doubal's (KD) method was found to be the most reliable predictor for mortality. Overall, the performance of biological age (BA) in mortality prediction was significantly better than using chronological age (CA). Cho et al 35] studied various BA estimation methods to examine the relation with 
work ability index (WAI). WAI is a measure that reflects present health condition rather than how it changes with age and their analysis showed that the KD method on PCA features produced the most reliable results. Mitnitski et al 97 compared the performance of the frailty index (FI) with biomarker-based measures of BA. They employed the KD algorithm in predicting mortality. The problems of estimating biological is still exists and it is two fold: first how to estimate efficiently, and then how to quantify whether the estimated biological age is better or worse than the existing methods.

\subsubsection{Quantifying Biological Age Using Physical Activity}

A recent popular study [63 shows that more than $27.5 \%$ of adults had insufficient physical activity worldwide. The study included 358 population-based surveys in 168 countries with a total of 1.9 million participants [11]. Numerous health risks such as hypertension, diabetes, mental health, and weight-gain are related directly to physical activity 63]. With aging, intensity of physical activity decreases/reduces for older people 32,134, and this is more evident for females [42]. Various organizations [8,11] have recommended levels of physical activity for different age groups. However, the exact relationship between physical activity and aging is still unclear $[115]$. For instance, there is still the question of whether physical activity can predict age 43 .

\subsection{Thesis Contribution}

Below we describe the key contributions of this thesis in addressing the problems discussed above.

\subsubsection{Obesity and Health Risk.}

We argue that the distribution of body weight, rather than the absolute weight, is a key factor in predicting health risk. A person with much of the body weight around the midsec- 
tion is at a much greater risk of disease and early mortality, when compared with another person that has weight better distributed peripherally (especially in lower body) [19]. This observation relates to the so-called 'apple-shaped vs. pear-shaped' phenomena, whereby the waist-to-hip ratio (WHR) is used to determine whether a person is apple-shaped, or pearshaped. While indices such as BMI, ABSI, waist-to-height ratio (WHtR) measure body shape, others such as BSA, WC, H, and VTC provide some indication of body size. The body surface area (BSA) provides a measure of the body size, while the VTC measures both the body size, and body shape. In this work, we consider both body shape and body size simultaneously, and thus combine the BSA and VTC with height and WC to develop a new surface-based body shape index. we define the Surface-based Body Shape Index (SBSI) as

follows: $S B S I \simeq \frac{\left(H^{7 / 4)}\left(W C^{5 / 6}\right)\right.}{B S A V T C}$. We then showed the performance of the SBSI: in terms of the SBSI increases generally linearly with age, that the relative death rate increases almost exponentially with increasing values of SBSI, and that SBSI shows substantial improvements in mortality modeling, when compared with popular body shape indices.

\subsubsection{Chronological Age from Body Shape.}

Using anthropometric measurements does not directly lead to accurate age prediction. We propose a multi-stage age estimation framework, whereby we first estimate the age group, and then for each age group, we construct learning models suited for the body shape types found at that age group. The goal is to investigate whether anthropometric measurements or combination of measurements can lead to improved age estimation. In the first step we perform eigen body shape modeling based on principal component analysis (PCA) and graph Laplacian. We use the spectral features obtained from the transformations. In the next step we group the subjects based on their shape features using $k$-means clustering. Once we have the shape clusters, for each cluster we compute an initial estimate of a person's age and perform age grouping based on this initial estimate. In the final stage now we perform (a) second-level age estimation, and (b) refinement of age estimates using neighborhood 
contexts. We then perform a cross database evaluation by training on a dataset and testing on a different dataset.

\subsubsection{Biological Age via Age Neighborhoods.}

We propose a neighborhood-based method to estimate biological age using biomarkers. We consider each subject as a point in the multidimensional feature space defined by the individual features. We compute centroid for each age based on the training dataset. Then we calculate the mean and standard deviation of each feature for all the age groups. At the testing stage, we first determine the age neighborhoods for an individual based on his features and the precomputed age centroids. Then we estimate the biological age using the age neighborhoods. We show two approaches based on the centroid and standard deviation. Similar to the centroid of mean, we also consider median based biological age estimation along with inter quartile range. For comparison of the proposed BA algorithms, we then apply three statistical analysis methods, namely, Cox proportionality hazard model, Kaplan Meier curves along with log-rank test, and survival area under the curve of receiver oper-

ating characteristics. Results from these methods suggest that the proposed centroid-based and median-based are competitive biological age predictor, and both the method showed improvement over all the other existing methods.

\subsubsection{Deep Learning for Biological Age Analysis Using Locomotor Physical Activity}

We used physical activity data from the National Health and Human Nutrition Examination Surveys (NHANES) 2003 - 2004 and 2005 - 2006. NHANES provides physical (locomotor) activity for a 7-day continuous tracking of "activity counts" that is sampled every minute and recorded using a physical activity monitor (ActiGraph AM-7164 piezoelectric accelerometer). Given the nature of the time series human locomotor data, we applied moving averages for smoothing the dataset (e.g., exponentially weighted moving average). We also applied the 
Box-Cox transformation [25]. The Box-Cox transformation is a point transformation defined as $y_{\lambda}(x)=\left(x^{\lambda}-1\right) / \lambda$, if $\lambda \neq 0$ or $y_{\lambda}(x)=\log (x)$ if $\lambda=0$.

Our proposed method for estimating biological age is to apply the combination of LSTM with CNN to the human physical locomotor activity data. We take advantage of the structure in the sequence of $2 \mathrm{D}$ representations of the daily activities to learn valuable patterns from the activity data (which is very difficult using 1D CNN, LSTM, or DNN). We consider the data as a temporal 7(D) day information. Where each day has 24 hours and an hour is 60

minutes. So to break it down, we represent it as a two dimensional information of $24 \times 60$ (HxM) minutes with a temporal information of 7 days. The three dimensional information is therefore, $7 \times 24 \times 60(\mathrm{DxHxM})$ minutes of data. This representation makes it easier to identify repeated temporal patterns in the data, which might provide cues to the functional or health status of the individual and thus to their biological age. We also demonstrate five deep biological age estimation models including the proposed approach and compare their performances on the NHANES physical activity data.

\subsubsection{Publications from PhD Work}

- SA Rahman, P Giacobbi, L Pyles, C Mullett, G Doretto and D Adjeroh, "Deep Learning for Biological Age Estimation", Briefings in Bioinformatics, 2019 (in review).

- SA Rahman, D Adjeroh. "Estimating Adult Age from Human Body Shape", PLoS ONE, 2019 (in review).

- SA Rahman, D Adjeroh. "Deep Learning using Convolutional LSTM estimates Biological Age from Locomotor Physical Activity", Nature Scientific Reports, 2019. ([link])

- SA Rahman, D Adjeroh. Centroid of Age Neighborhoods: A New Approach to Estimate Biological Age", IEEE Journal of Biomedical and Health Informatics, 2019. ([link])

- SA Rahman, D Adjeroh. "Surface-Based Body Shape Index and Its Relationship with All-Cause Mortaltiy", PloS One 10 (12), 2015 [link] 
- SA Rahman, D Adjeroh, "Estimating Biological Age from Physical Activity using Deep Learning with 3D CNN", IEEE International Conference on Bioinformatics and Biomedicine, 2019.

- SA Rahman, D Adjeroh, "Centroid of Age Neighborhoods: A Generalized Approach to Estimate Biological Age", 2019. The IEEE-EMBS International Conference on Biomedical and Health Informatics [link].

- AHM Rubaiyat, TT Toma, M Kalantari, SA Rahman, Yanfang Ye, "Automatic Detection of Helmet Uses for Construction Safety", Proceedings of the IEEE/WIC/ACM WI Workshop on Advanced Methods in Optimization and Machine Learning (WIBIH)[link], 2016.

- SA Rahman, T Afrin, D Adjeroh, "Determinants of User Ratings in Online Business Rating Services", International Conference on Social Computing, Behavioral-Cultural Modeling, $2015[$ link] ,

\subsection{Organization}

The dissertation is organized as follows. In Chapter 2, we introduce a new anthropometric measure called Surface-based Body Shape Index (SBSI) that accounts for both body shape and body size, and evaluate its performance as a predictor of all-cause mortality. Chapter 3 presents a new multi-stage approach, for chronological age prediction based on human body measurements. We investigate whether human body shape can be exploited for reliable age estimation for adult humans. Chapter 4 proposes a new centroid-based biological age estimation method using blood biomarkers. In Chapter 5 we introduce a deep learning approach for biological age estimation. In particular, we propose a method to predict biological age using

human physical activity based on deep convolutional long short term memory. Chapter 7 describes possible directions for future work. 


\section{Chapter 2}

\section{Surface-based Body Shape Index}

Obesity, with its dual complications of diabetes mellitus and cardiovascular disease (CVD), has emerged as a major public health challenge [50,98, 105]. Obesity is identified by the World Health Organization (WHO) as a global epidemic [141. In the US, obesity prevalence remains high at $35.7 \%$ of the adult population [104, while $68 \%$ are classified as obese or overweight [50], with the highest rates being found among the populations that are poor, have lower education, and are minority groups [50]. The picture for childhood and adolescent obesity is no better, with $16.9 \%$ obesity prevalence, and $31.8 \%$ classified as obese or overweight [104], and thus at the risk of developing insulin resistance, dyslipidemia, or hypertension at an early age 117 . This trend is mirrored by the high incidence of diabetes, which has shown a similarly high prevalence rates [5]. The problem of obesity is attributed to the issue of imbalance between energy intake and energy expenditure in the body [116]. The problem is directly connected to the quantity of adipose depots (body fat). Adiposity is associated with increased risk of many chronic diseases in the general population 27, 34, 37, 47, 81. Obesity is known to be associated with diabetes, and various forms of cardiovascular disease (CVD). Other associated complications include depression, mobility issues, some forms of cancer [55], sleep apnea [107], osteoarthritis, among others (see [116] for a review). 
Many different anthropometric measures have been used to assess adiposity. The body mass index (BMI) is one of the best known indices of relative adiposity or excess body weight in the association of body composition with mortality. Individuals are often grouped into BMI categories [52, 141] (underweight $(B M I<18.5)$, normal weight $(18.5 \leq B M I<25)$, overweight $(25 \leq B M I<30)$, obese I $(30 \leq B M I<35)$, obese II $(35 \leq B M I<40)$, and obese III $(B M I \geq 40))$. Risk of CVD and diabetes tends to increase with increasing BMI. The association of BMI with mortality in the general population is usually found to exhibit a U-shaped [15,51] or J-shaped [90,95] curve. Using BMI-defined categories Flegal et al [51] showed, that obese and underweight individuals had a higher death rate, while normal weight and overweight individuals had a similar relative mortality risk. Some have hypothesized that the non-linear relationship observed between BMI and mortality may be a consequence of BMI being a composite of both fat and fat-free mass [14, 22, not simply a surrogate for overall adiposity. These observations point to the core limitation of BMI as a measure of adiposity. Several studies have shown that adjustment for waist circumference, a surrogate for abdominal adiposity [66, 75, 131], eliminates or attenuates BMI's nonlinear relationship with mortality [23, 79]. The ABSI (A Body Shape Index, defined as $A B S I=W C /\left(B M I^{2 / 3} * H^{1 / 2}\right)$ which places more emphasis on waist circumference was proposed by Krakauer and Krakauer [85] as an alternative to the BMI, resulting in a better prediction of mortality hazard. Various other one dimensional (1D) anthropometric measures (e.g., waist circumference (WC), hip circumference (HC), skin folds (SFs)), and their relation to obesity have also been studied. Example, the waist-to-hip ratio (WHR) is a better indicator of ischemic heart disease mortality [99], while the waist-to-height ratio (WHtR) provides a better predictor for death, heart attack and stroke [126]. Ohrvall et al [106] and Pouliot et al [112], showed sagittal abdominal diameter to be a better measure of the accumulation of visceral adipose tissue and cardiovascular risk. Beyond 1D measures, there are also studies linking obesity-related diseases with 2D measures (e.g., body surface area (BSA) [146]), and 3D measures 135, 138, 140], (e.g., body volume index (BVI) 222,123]). 
Results in 85 showed that ABSI produced better results than both BMI and WC in terms of all-cause-mortality hazard prediction. More recent studies, however, show that ABSI does not perform better than WC for diabetes mellitus (DM) prediction [68]. He et al [68], showed that for the Chinese population, the three measures WC, BMI, and ABSI showed similar predictive abilities. Zhang et al [148], showed ABSI to be a weak predictor for the risk of cardiovascular diseases (CVD), or the problem of Metabolic Syndrome (MetS). Clearly, no single measure can capture all aspects of the general problem of obesity and its related diseases. In this work, we first introduce a new anthropometric measure (called Surfacebased Body Shape Index, SBSI) that accounts for both body shape and body size. Then, we evaluate the proposed measure as a predictor of all-cause-mortality, and compare its performance with other popular body shape indices, namely BMI, WC, and ABSI.

\subsection{Materials and Methods}

\subsubsection{Datasets.}

We used mortality data combined with anthropometric data from the National Health and Human Nutrition Examination Surveys (NHANES) 1999-2004 [3,4,6]. NHANES employs a complex cluster design to sample members of the civilian USA population who are not institutionalized. NHANES uses stratified multistage probability to sample the data. Mortality information from public-use mortality files is linked to the National Death Index (NDI). Since not all the data contained mortality information we excluded those individuals that do not have data on mortality. Ethnicity included white, black, Mexican and others. Anthropometric measurements included BMI, height, weight, and waist circumference. We used the NHANES mobile examination center sample. The mobile examination center used trained examiners who used standardized protocols to measure the anthropometric parameters. Mortality data based on NDI were available in 2006. After refining, we obtained 11,808 
individuals with 701 deaths during the 2-8 years of follow-up (1999-2006).

We also used data from the Civilian American and European Surface Anthropometry Resource (CAESAR) 122. CAESAR project was a survey of the civilian populations from four countries namely the United States of America (USA), Canada, the Netherlands, and Italy. The survey was carried out by the U.S. Air Force, resulting in complete 3-D models of each civilian subject. The 3-D surface anthropometry was performed using three scanned poses using the cyberware 3D whole-body scanner [1]. The CAESAR dataset also includes manual hand measurements of the various anthropometric attributes, recorded as 1D information. For our purpose, we used the 1D datasets from the CEASAR survey, which contains 2400 US and Canadian civilians, ages 18-65 (http://store.sae.org/caesar/). We selected 45 key human body measurements, as reported by Adjeroh et al 12 and Cao et al [30]. From our analysis, the key measurements shared by both datasets tend to have similar general statistics. For example, the mean and standard deviation were observed as follows: height (NHANES $167.7 \pm 10.1$; CAESAR $170.46 \pm 10.2$ ), waist circumference (NHANES $92.2 \pm 13.2$; CAESAR $84.8 \pm 14.4$ ), weight (NHANES $74 \pm 15.8$; CAESAR 77 \pm 19.8 ), BMI (NHANES $26.2 \pm 4.7$; CAESAR $26.3 \pm 5.7$ ). Apart from the prediction of VTC for NHANES samples based on learned parameters from CAESAR, all other analyses are based on the NHANES dataset.

\subsubsection{Study Variables.}

In the CAESAR study [122], data collection was a three-step process: in-processing/demographics; traditional hand measurements with tape and calipers; and 3D whole-body scanning stations. All measurements were taken with participants wearing light clothes and without shoes. For height, subject stands fully erect with weight distributed equally on both feet, with both arms hanging freely downwards. Thigh circumference was measured on a seated subject. Triceps skinfold is the thickness of the skinfold overlaying the triceps muscle. This was measured on the back of the upper arm, between the tip of the shoulder and the elbow while 
the subject's arm is bent $90^{\circ}$. VTC was measured using a tape from the shoulder, through the crotch, and back to the shoulder while the subject stands fully erect with the weight distributed equally on both feet and the arms hanging freely downwards. Waist circumference is the maximum circumference of the waist that can be measured using a tape measure, which starts at the top of subject's hip bone, then all the way around level with his/her belly button. Height, circumferences, and length measurements were made to the nearest $0.1 \mathrm{~cm}$, while weight was measured to the nearest kilogram.

In the NHANES study [3, 4, 6], anthropometric measurements were taken by trained personnel. Height was obtained using a digital meter. Subjects wore a light examination gown before measuring their weight on a digital scale. Waist circumference was measured just above the uppermost lateral border of the ilium. A key component of the proposed SBSI is the vertical trunk circumference (VTC). Given that NHANES does not contain information on the VTC for its subjects, we first learned the regression parameters for predicting the VTC using the CAESAR dataset. Then, we applied the learned parameters on the samples from NHANES to predict their VTC. Since the two datasets have similar overall statistics, we can rely on the results of the prediction for subjects in NHANES. Based on the measures, we computed the BMI using the standard formula: $B M I=W / H^{2}$ (unit $k g / m^{2}$ ), where $\mathrm{W}$ is weight $(\mathrm{kg})$, and $\mathrm{H}$ is height $(\mathrm{m})$. The body surface area (BSA) is assessed following Shuter and Aslani [130]: $B S A=0.00949 \times W^{0.441} \times H^{0.655}$. BMI obesity categories were computed following WHO definitions $[56,62]$ : underweight $(B M I<18.5)$, normal $(18.5 \leq B M I<25)$, overweight $(25 \leq B M I<30)$, obese I $(30 \leq B M I<35)$, obese II $(35 \leq B M I<40)$, and obese III $(B M I \geq 40)$.

\subsubsection{Surface-based Body Shape Index (SBSI).}

The BMI provides a simple coarse measure of the body shape. Two people in the same BMI category could have very different body shapes, and different body sizes. The distribution 
of body weight, rather than the absolute weight, is a key factor in predicting health risk. A person with much of the body weight around the midsection is at a much greater risk of disease and early mortality, when compared with another person that has weight better distributed peripherally (especially in lower body) [19]. This observation relates to the socalled 'apple-shaped vs. pear-shaped' phenomena, whereby the waist-to-hip ratio (WHR) is used to determine whether a person is apple-shaped ( $W H R<0.8$ for women, $W H R<0.9$ for men), or pear-shaped ( $W H R \geq 0.8$ for women, $W H R \geq 0.9$ for men). See [19]. The waist circumference (WC) is often combined with the BMI for an improved assessment of body shape [85]. Other studies used waist-to-height ratio (WHtR) as a shape index [109]. In addition to body shape, the body size is also another important factor. While indices such as BMI, ABSI, waist-to-height ratio (WHtR) measure body shape, others such as BSA, WC, $\mathrm{H}$, and VTC provide some indication of body size. The body surface area (BSA) provides a measure of the body size, while the VTC measures both the body size, and body shape. In this work, we consider both body shape and body size simultaneously, and thus combine the BSA and VTC with height and WC to develop a new surface-based body shape index.

To investigate the significance of the BSA and VTC, we analyzed their relationship with height and waist circumference, for a given BMI category, using the NHANES dataset. The results are shown in Fig. 2.1. It can be observed that, at a given height, obese individuals tend to have higher BSA, while those that are underweight tend to have a lower BSA. At a given height, the BSA tends to increase steadily with BMI (Fig. 2.1 ) . VTC and height show a similar behavior at given BMI categories (Fig. 2.1b). The relationship between BSA and WC (or VTC and WC) is not as clear. Unlike the clear linear association between BSA (or VTC) and height, for a given BMI category, BSA (VTC) has a non-linear relationship with WC, for a given BMI. Yet, the different BMI categories are evident from the graphs (Fig. 2.1c, d). The underweight group clustered in mainly the bottom left quadrant, while the obese III category clustered around the top right quadrant. 


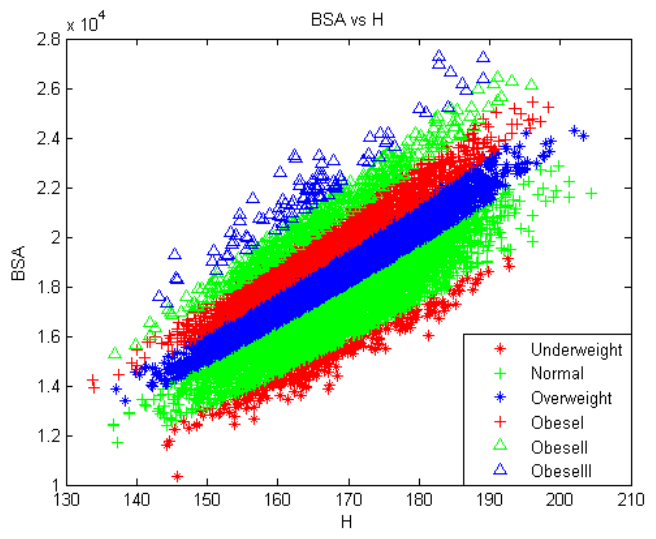

(a)

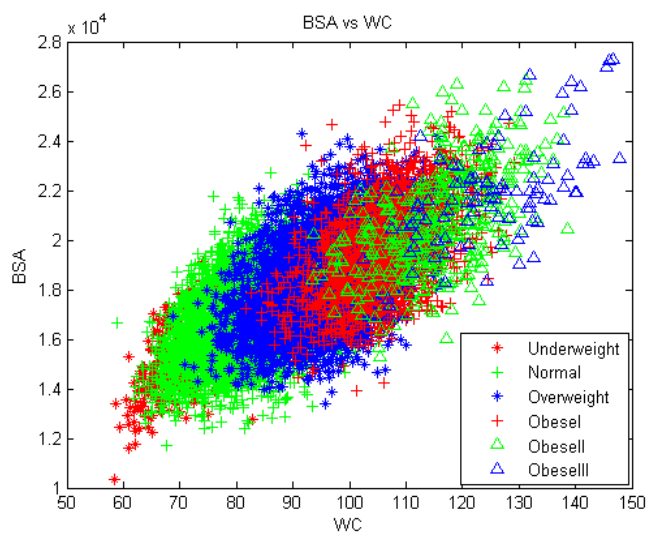

(c)

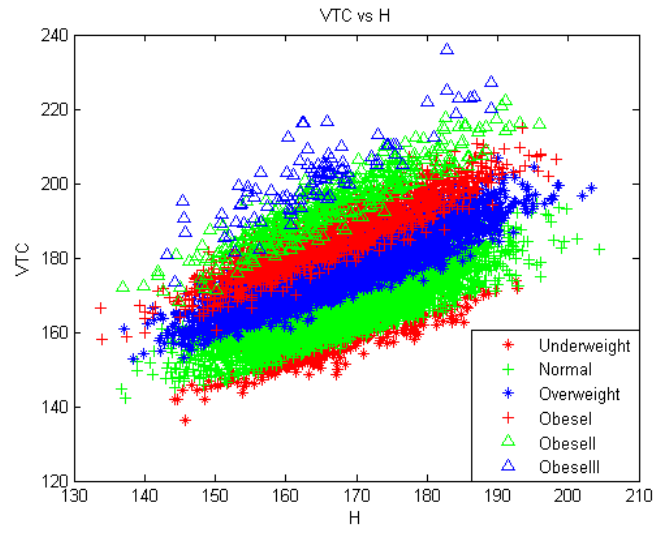

(b)

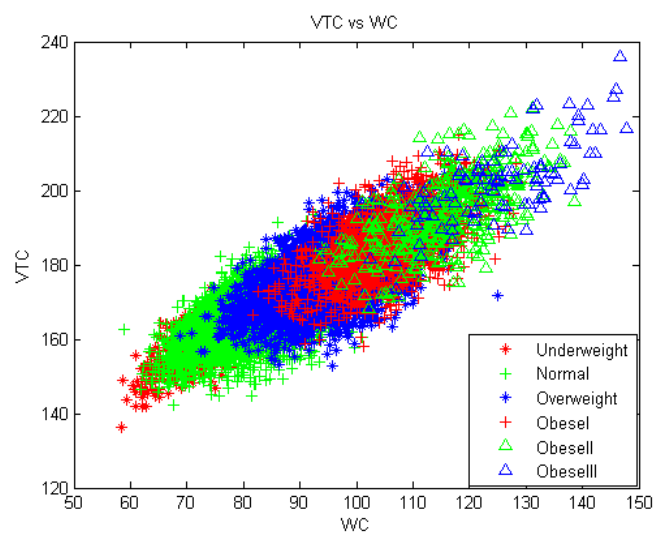

(d)

Figure 2.1: Relationship between BSA, VTC, height and WC for given BMI categories. The BSA and height, and VTC and height can predict the BMI categories (a, b). BSA and WC (and VTC and WC) show a non-linear relationship for a given BMI category (c, d). 


\subsubsection{SBSI Construction.}

Different formulae have been proposed for estimating the body surface area (BSA), based mainly on the weight and height. In a survey of different BSA predictors [40], the Shuter and Aslani method [130] was shown to provide an overall best performance. Thus, in this work, we adopt this method to predict BSA as follows:

$$
B S A=0.00949 \times W^{0.441} \times H^{0.655},
$$

where $\mathrm{W}=$ weight in kilograms, and $\mathrm{H}=$ height in meters. For VTC, we first identified common anthropometric measurements between the CAESAR and NHANES datasets. Then we performed simple linear regression using the samples in CAESAR and subsequently applied regression learning to predict the VTC for the samples in the NHANES database. The VTC (in $\mathrm{cm}$ ) is predicted using the formula:

$$
\begin{aligned}
V T C= & (61.2 \pm 2.81)+A C(0.315 \pm 0.03)+H(0.409 \pm 0.01)+S S(0.237 \pm 0.13)- \\
& T C(0.089 \pm 0.02)-T S(0.12 \pm 0.13)-U A L(0.453 \pm 0.05)+W C(0.137 \pm 0.01)+ \\
& W(0.37 \pm 0.02)
\end{aligned}
$$

where $\mathrm{AC}=\operatorname{arm}$ circumference $(\mathrm{cm}), \mathrm{H}=$ height $(\mathrm{cm}), \mathrm{SS}=$ subscapular skin fold $(\mathrm{cm})$, $\mathrm{TC}=$ thigh circumference $(\mathrm{cm}), \mathrm{TS}=$ triceps skinfold $(\mathrm{cm}), \mathrm{UAL}=$ upper arm length $(\mathrm{cm})$,

$\mathrm{WC}=$ waist circumference $(\mathrm{cm}), \mathrm{W}=$ weight $(\mathrm{kg})$. This prediction resulted in error measure $\left(R^{2}=0.9217\right.$, p-value $\left.(\mathrm{P})=2.2 \times 10^{-16}\right)$ using the CAESAR dataset.

We then combine the BSA and VTC with the height and waist circumference by using linear regression on $\log (B S A \times V T C)$ as a function of $\log (H)$ and $\log (W C)$ for the entire database:

$$
\log (B S A \times V T C)=(0.922 \pm 0.02)+(1.766 \pm 0.009) \log (H)+(0.838 \pm 0.003) \log (W C)
$$


The error measure for this prediction was $\left(R^{2}=0.9, P=2.2 \times 10^{-16}\right)$. From the above, we can infer the relationship:

$$
(B S A \times V T C) \propto\left(H^{7 / 4}\right)\left(W C^{5 / 6}\right)
$$

Taking ratios of the two sides, we define the Surface-based Body Shape Index (SBSI) as follows:

$$
S B S I \triangleq \frac{\left(H^{7 / 4)}\left(W C^{5 / 6}\right)\right.}{B S A V T C}
$$

\subsubsection{Statistical Analysis.}

We analyzed the data separately for male and female subjects, and for their combination. Table 3.1 shows the characteristics of the study participants for NHANES dataset. The corresponding data for the CAESAR dataset is provided as Supplementary Material in Table 2.7. The sample mean for SBSI (using NHANES) is 0.10718 \pm 0.00627 , with a minimum of 0.08218 , and a maximum of 0.14228. For CAESAR dataset, we observed mean SBSI of $0.10644 \pm 0.006092$, a minimum of 0.07437 , and a maximum of 0.1386 .

Table 3.2 shows the correlation between the SBSI and other anthropometric body indices. The table shows the correlation using direct measurements for both Pearson's $\rho$ (upper half), and Kendall's $\tau$ (lower half). For a given measurement value $x$, its $z$-score is computed as $z(x)=(x-\mu) / \sigma$, where $\mu$ and $\sigma$ are the mean and standard deviation for the measurement. SBSI has high correlation with ABSI, low correlation with WC and height, and negative correlation with BMI and weight. The reason $A B S I=\frac{W C}{\left(B M I^{2 / 3} \times H^{1 / 2}\right)}$ has a high correlation with SBSI might be because of the fact that both ABSI and SBSI use WC, and H in similar roles in their respective formulae.

We used Cox proportionality mortality hazard modeling [39, 84 to quantify the association 
Table 2.1: Key anthropometric attributes for study participants.

\begin{tabular}{|c|c|c|c|c|c|c|}
\hline & \multicolumn{2}{|c|}{ All $(\mathrm{N}=11808)$} & \multicolumn{2}{|c|}{ Female $(\mathrm{N}=5840)$} & \multicolumn{2}{|c|}{ Male $(\mathrm{N}=5968)$} \\
\hline & Average & SD & Average & SD & Average & SD \\
\hline Age(years) & 45.692 & 20.726 & 45.418 & 20.920 & 45.959 & 20.532 \\
\hline Weight $(\mathrm{W})(\mathrm{kg})$ & 74.015 & 15.801 & 67.905 & 14.065 & 79.994 & 15.105 \\
\hline Height $(\mathrm{H})(\mathrm{cm})$ & 167.726 & 10.102 & 160.936 & 7.136 & 174.371 & 7.925 \\
\hline $\operatorname{BMI}\left(k g / m^{2}\right)$ & 26.227 & 4.696 & 26.201 & 5.065 & 26.253 & 4.306 \\
\hline Leg Length $(\mathrm{cm})$ & 40.561 & 3.790 & 38.638 & 3.217 & 42.443 & 3.337 \\
\hline Arm Length $(\mathrm{cm})$ & 37.088 & 2.731 & 35.489 & 2.137 & 38.654 & 2.309 \\
\hline Arm Circumference $(\mathrm{cm})$ & 31.262 & 4.072 & 30.084 & 4.102 & 32.415 & 3.694 \\
\hline Waist Circumference $(\mathrm{cm})$ & 92.190 & 13.229 & 89.573 & 13.035 & 94.752 & 12.914 \\
\hline Thigh Circumference $(\mathrm{cm})$ & 51.362 & 5.984 & 50.921 & 6.346 & 51.793 & 5.573 \\
\hline Triceps Skinfold $(\mathrm{cm})$ & 17.675 & 8.066 & 22.387 & 7.235 & 13.064 & 5.875 \\
\hline Subscapular skinfold(cm) & 19.072 & 7.566 & 20.049 & 7.848 & 18.116 & 7.152 \\
\hline Vertical Trunk Circumference(VTC)(cm) & 174.924 & 11.908 & 170.349 & 10.845 & 179.402 & 11.177 \\
\hline A Body Shape Index(ABSI $)\left(m^{11 / 6} k^{-2 / 3}\right)$ & 0.081 & 0.005 & 0.080 & 0.006 & 0.081 & 0.005 \\
\hline Body Surface Area(BSA) $\left(\mathrm{cm}^{2}\right)$ & 18074 & 2168 & 16927 & 1759 & 19196 & 1930 \\
\hline WHtR & 0.551 & 0.080 & 0.557 & 0.084 & 0.544 & 0.075 \\
\hline Surface Based Body Shape(SBSI) & 0.107 & 0.006 & 0.107 & 0.007 & 0.108 & 0.006 \\
\hline
\end{tabular}

Table 2.2: Correlation coefficient between anthropometric measures.

\begin{tabular}{|l|l|l|l|l|l|l|l|l|}
\hline & $\mathrm{H}$ & $\mathrm{W}$ & $\mathrm{BMI}$ & $\mathrm{WC}$ & $\mathrm{VTC}$ & $\mathrm{BSA}$ & ABSI & SBSI \\
\hline $\mathrm{H}$ & 1 & 0.548 & -0.023 & 0.194 & 0.568 & 0.761 & -0.007 & -0.025 \\
\hline $\mathrm{W}$ & 0.385 & 1 & 0.817 & 0.826 & 0.975 & 0.958 & 0.079 & -0.406 \\
\hline BMI & -0.005 & 0.612 & 1 & 0.855 & 0.778 & 0.626 & 0.091 & -0.475 \\
\hline WC & 0.129 & 0.620 & 0.668 & 1 & 0.838 & 0.708 & 0.542 & -0.023 \\
\hline VTC & 0.390 & 0.864 & 0.576 & 0.634 & 1 & 0.948 & 0.163 & -0.357 \\
\hline BSA & 0.561 & 0.824 & 0.435 & 0.499 & 0.791 & 1 & 0.062 & -0.323 \\
\hline ABSI & 0.006 & 0.072 & 0.093 & 0.391 & 0.125 & 0.055 & 1 & 0.801 \\
\hline SBSI & -0.011 & -0.251 & -0.293 & 0.003 & -0.218 & -0.197 & 0.583 & 1 \\
\hline
\end{tabular}

Pearson's $\rho$ (upper half), and Kendall's $\tau$ (lower half). 
of the proposed SBSI and other anthropometric measures (ABSI, BMI, and WC) with allcause mortality. Under the Cox model, the relationship between hazard and the covariates is described by considering the logarithm of the hazard as a linear function of the variables. Following the Poisson model, this can be expressed by using exponentiation on the covariate terms 39:

$$
h(t, x)=\exp \left(\beta_{0}+\beta_{1} x\right)=h_{0} \exp \left(\beta_{1} x\right)>0
$$

where, $h_{0}$ is the baseline hazard, $\beta_{0}$ and $\beta_{1}$ are coefficients influencing the covariates $x$. This is often generalized as follows:

$$
h(t, x)=h_{0}(t, \alpha) \exp \left(\beta^{T}, x\right)
$$

where $\alpha$ are the parameters influencing the baseline hazard. In our approach we modeled the log death rate as a nonparametric function of time (months of follow-up from the interview) and coefficients are fitted which multiply the value of the predictor variables. Although predictors can be entered as either continuous or discrete, we used predictor's $z$-score as continuous variables for generalization. Previous studies suggest that using $z$-score in the hazard model produce better results 85. We calculated mortality risk associated with each anthropometric measurement separately for male and female subjects, and later for all subjects in the dataset. Then we divided the dataset using BMI categories to test the range of applicability of our proposed SBSI and also how it compares with other existing body shape indices. We used the $R^{2}$ statistic to measure how successful the model is in explaining the variation of the data.

To further study the predictive capabilities of SBSI and to compare with other body shape indices, we constructed and analyzed the Kaplan-Meier (KM) curves [80] using each measure. The Kaplan-Meier estimate of the survival function is a non-parametric method of estimating survival from data. It is very popular because it makes only very weak assumptions about the data. In medical research, it is used to measure the fraction of patients surviving for a certain amount of time after treatment. Let $S(t)$ be the probability that a member from a 
given population will have a lifetime exceeding t. For a sample of size $N$ from this population, let the observed times until death of the $N$ sample members be $t_{1} \leq t_{2} \leq t_{3} \leq \ldots \leq t_{N}$. Corresponding to each $t_{i}$ is $n_{i}$, the number "at risk" just prior to time $t_{i}$, and $d_{i}$, the number of deaths at time $t_{i}$. The Kaplan-Meier estimator is the nonparametric maximum likelihood estimate of $\hat{S}(t)$, where $\hat{S}(t)$ is a product of the form

$$
\hat{S}(t)=\prod_{t_{i} \leq t} \frac{n_{i}-d_{i}}{n_{i}},
$$

We performed analysis using KM survival curve estimates for all the data, and separately for all female, and all male. Then we did more rigorous study based on BMI categories. We used the log-rank test to compare the survival distributions obtained using different shape indices. The log-rank test tries to distinguish between Kaplan-Meier curves to see if they are statistically equivalent. The output of the test is a $\chi^{2}$-distance, and the Pvalue associated with the distance. Higher $\chi^{2}$-distances and low P-values indicate a better separation between the curves, and hence a better performance in mortality modeling. All statistical analyses were performed using the $\mathrm{R}$ Language (ver. 3.0.3, The $\mathrm{R}$ Foundation for Statistical Computing, Vienna, Austria). We considered $P \leq 0.05$ to be statistically significant.

\subsection{Results}

\subsubsection{Higher SBSI with Increasing Age.}

The SBSI increases generally with increasing age. Fig. 2.2 shows how the SBSI and three other anthropometric measures vary with age for both male and female. Subjects in our NHANES dataset had age ranges between 18-85 years. Mean SBSI increases consistently (generally linearly) for male subjects. However, for female subjects, the mean goes up from about ages 18 to 24 then goes down till about age 30; between ages 30 and 40, the mean SBSI did not have a definite pattern for females, going up and then down. After this point, the SBSI generally increased with age. For WC, ABSI and SBSI, the mean values for males were 
clearly separated from those for females, with the value for males being generally greater. For the BMI, this distinction was not as clear. Unlike ABSI and SBSI that had a generally linear relationship with age, the BMI and WC had an inverted U-like shape, with the turning points around age 75 for WC, and age 70 for BMI.



(a) $\mathrm{WC}$

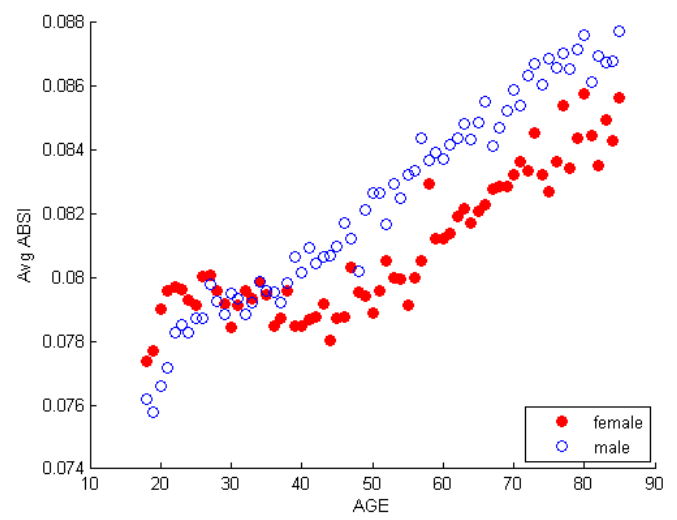

(c) ABSI

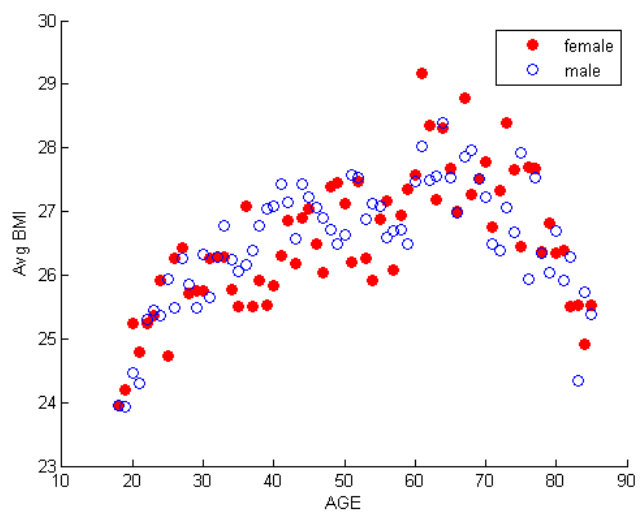

(b) BMI

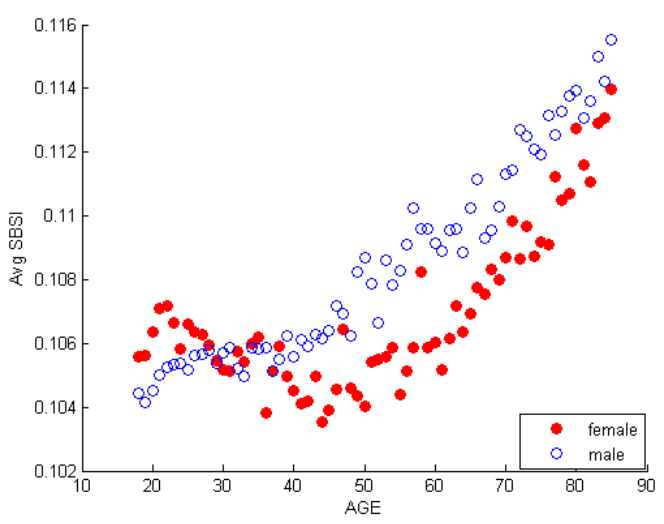

(d) SBSI

Figure 2.2: Variation of different body shape indices with age (in years).

\subsubsection{Higher Mortality Hazard for Increasing SBSI.}

Fig. 2.3 shows the variation of the relative death rate with SBSI using their $z$-scores. The figure shows that the relative death rate increases almost exponentially with increasing values of SBSI. The variability in the mortality hazard prediction also seems to increase with 
increasing SBSI values. The results in this figure are consistent with known results that relative death rate is generally higher for male than female subjects. In Fig. 2.3(a) the relative death rate for female was almost similar until about the 50th percentile (average 1.3) then it went up (from 4 to 14). For male (Fig. 2.3b) average death rate was 1.02 until about the 35th percentile, after that it grew exponentially (from 3 to 28).

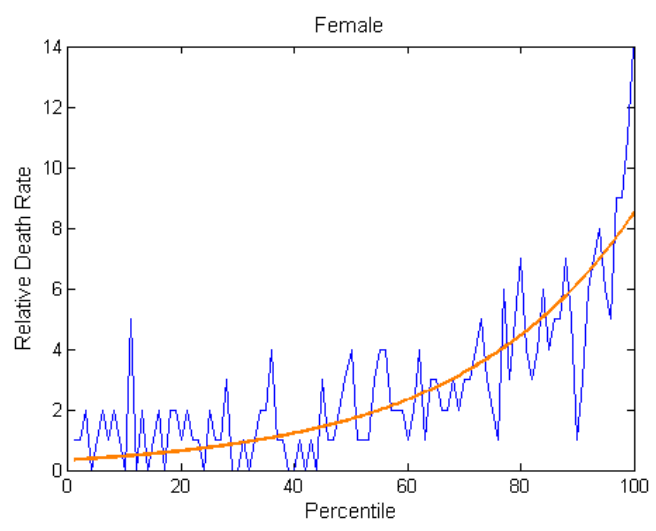

(a)

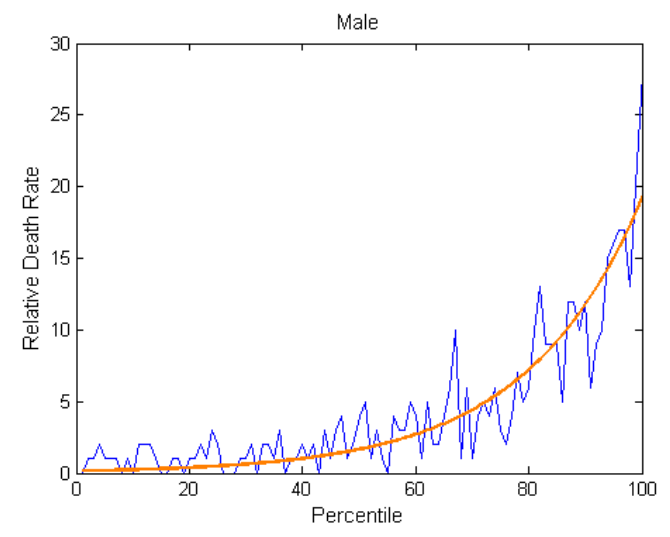

(b)

Figure 2.3: Variation of relative death rate with increasing values of SBSI z-score. (a) Female; (b) Male.

\subsubsection{Improved Modeling for All-Cause Mortality using SBSI.}

The proposed surface-based body shape index shows substantial improvements in mortality modeling, when compared with popular body shape indices. Table 3.3 shows the summary performance in mortality hazard modeling for SBSI, ABSI, BMI, and WC. The hazard ratio (HR) for SBSI was 2.287 for all, 2.019 for female and 2.456 for male. For all measures, the results are based on using their $z$-scores as a continuous variable, rather than the original value.

Table 2.4 shows the corresponding results in terms of the $\chi^{2}$-distance when using the logrank test to analyze the KM survival curves for each body shape index. The $\chi^{2}$-distance for SBSI was 570 for all, 147.68 for female and 434.372 for male. Here the analyses was done on the quartiles labeled as 1st Q, 2nd Q, etc. in Fig 2.4. From the table, SBSI performs significantly 
better than waist circumference and ABSI. Clearly, the BMI was unable to show a distinction in the survival rates for the quartiles, given its non-linear relationship with mortality-hazard.

Table 2.3: Summary of mortality hazard ratio for four key anthropometric measures.

\begin{tabular}{|l|l|l|l|}
\hline & ALL & Female & Male \\
\hline$B M I$ & $0.915(0.849-0.987)$ & $1.019(0.9148-1.136)$ & $0.823(0.738-0.9172)$ \\
\hline$W C$ & $1.327(1.237-1.425)$ & $1.338(1.195-1.498)$ & $1.254(1.14-1.378)$ \\
\hline$A B S I$ & $2.328(2.173-2.495)$ & $1.999(1.8-2.22)$ & $2.682(2.439-2.951)$ \\
\hline$S B S I$ & $2.287(2.142-2.443)$ & $2.019(1.809-2.253)$ & $2.456(2.269-2.658)$ \\
\hline
\end{tabular}

Results are reported as HR (95\% CI).

Table 2.4: Summary of $\chi^{2}$-distances for KM survival curves for four key anthropometric measures.

\begin{tabular}{|l|l|l|l|}
\hline & ALL & Female & Male \\
\hline$B M I$ & $2.20(0.531)$ & $1.0191 .345(0.718)$ & $6.064(0.108))$ \\
\hline$W C$ & $59.464\left(7.6510^{-13}\right)$ & $22.506\left(5.1210^{-5}\right)$ & $25.961\left(9.7210^{-6}\right)$ \\
\hline$A B S I$ & $551.126(0)$ & $141.697(0)$ & $415.643(0)$ \\
\hline$S B S I$ & $570.044(0)$ & $147.688(0)$ & $434.372(0)$ \\
\hline
\end{tabular}

Results are reported as $\chi^{2}$-distance (P-value).

Fig. 2.4 shows the detailed Kaplan-Meier curves for SBSI and three other key anthropometric body shape indices. A given variable is a good mortality predictor if the Kaplan-Meier curves are easily distinguishable (more distance between them), and the variable gives a reasonable performance from low to high levels, with less crossing between curves. SBSI performs very well in distinguishing the proportion of survivors over time (months) since examination. From the figure, it is clear that ABSI and SBSI are much better than WC and BMI in predicting survival, with the SBSI being slightly better than ABSI. The difference between ABSI and SBSI is more evident using quantitative measures, e.g., the $\chi^{2}$-distance between their respective KM curves, as captured by the logrank test (Table 2.4). More detailed results using the hazard ratio with BMI categories are described below (see Table 2.5). The corresponding results for using the log-rank test to analyze the KM plots are given in Table 2.6, Fig. 2.5 and Fig. 2.6. 


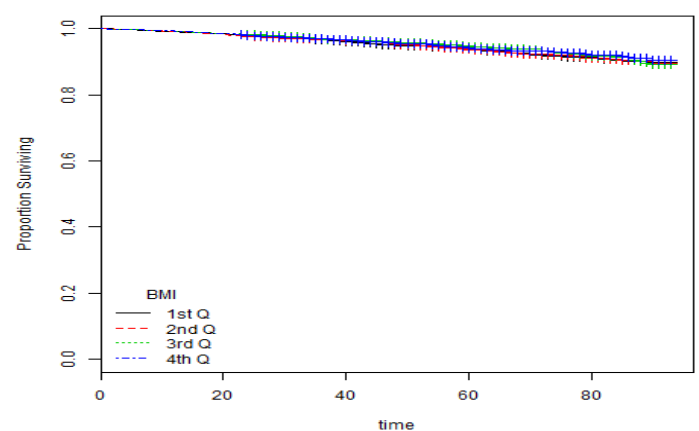

(a) BMI

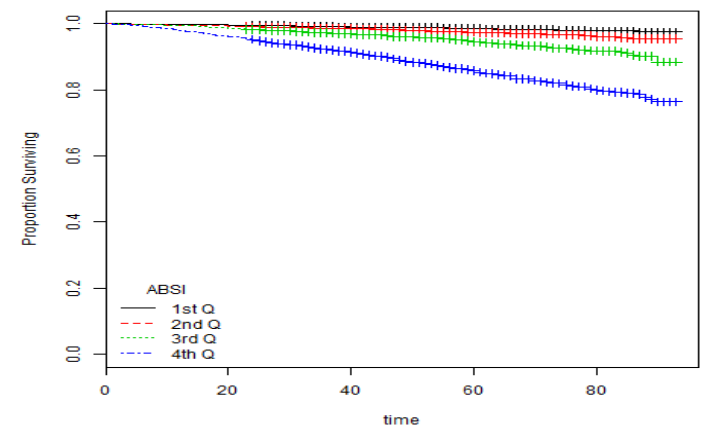

(c) ABSI

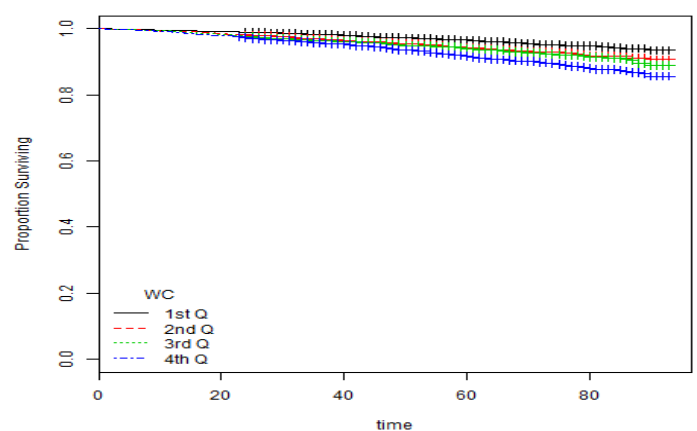

(b) WC

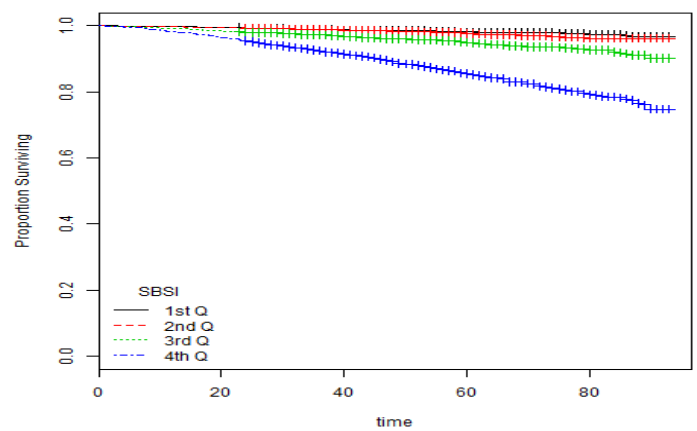

(d) SBSI

Figure 2.4: The Kaplan Meier curves for four body shape indices using all subjects. The SBSI shows a better prediction performance than other body shape measures (with more separation between the curves, and less crossovers). 1st Q, 2nd Q, etc. denote respectively 1st quartile, 2nd quartile, etc.

\subsubsection{Mortality Hazard using SBSI on BMI Categories.}

To further investigate the performance of SBSI in mortality modeling, we considered the mortality hazard ratio (HR) using the SBSI for each BMI category. Table 2.5 shows the results. In general, the hazard ratios using SBSI does not necessarily increase monotonically with increasing BMI values (BMI increases from underweight category to obese III). For instance, considering all subjects, mortality hazard ratio increased from $2.352(P<0.0001)$ for the underweight category, to $2.799(P<0.0001)$ for normal weight, and then decreased to $2.601(P<0.0001)$ for overweight, increasing again to $2.952(P<0.0001)$ for obese 
I. A similar trend is observed for female-only, and for male-only subjects. The table also shows the corresponding mortality hazard ratios using various other anthropometric shape indices. From the table, ABSI and the proposed SBSI tend to provide the best performance in most cases, followed by WC. Though ABSI provided the overall best result using all subjects (ABSI: HR 2.328, $P<0.0001$; SBSI: HR 2.287, $P<0.0001$ ), when split into BMI categories, SBSI provided a better performance over ABSI for all the BMI categories. SBSI was the overall best on each BMI category, except for underweight and normal weight categories (which had WC as the best performer). Using all subjects, ABSI performed better than SBSI on male subjects (ABSI: HR 2.682, $P<0.0001$; SBSI: HR 2.456, $P<0.0001$ ), while SBSI performed better on female subjects (ABSI: HR 1.999, $P<0.0001$; SBSI: HR 2.019, $P<0.0001)$. However, again when split into BMI categories, for male subjects, ABSI outperformed SBSI only in one category - obese II (ABSI: HR 4.900, $P=0.004$; SBSI: HR 4.080, $P<0.006)$. SBSI was the overall best in two categories, while $\mathrm{WC}$ was the overall best in two other categories. For female subjects, with BMI categories, SBSI provided the best results for 5 of the 7 categories, while WC reported best results for the other two categories. To further study the performance of SBSI and other anthropometric measures using BMI categories, we analyzed the Kaplan-Meier survival curves obtained using each measure, when applied separately to subjects in each BMI category. Table 2.6 shows the results of this analysis. Similar to the mortality hazard, SBSI logrank result ( $\chi^{2}$-distance) does not increase monotonically with increasing BMI. For example, considering all subjects, the $\chi^{2}$-distance increased from $11.947(\mathrm{P}=0.008)$ for the underweight category, to $296.451(P<0.0001)$ for normal weight and then decreased to $202.795(P<0.0001)$ for overweight, decreasing further to $130.434(P<0.0001)$ for obese I. We observe a similar trend for female-only, and for male-only subjects as well. The table also shows the results of the logrank test on other anthropometric shape indices. From the table, SBSI tends to provide the best performance in most cases followed by ABSI, WC. In general SBSI performed better than all the other anthropometric measures tested. Performing the logrank test for all subjects 
we get $\chi^{2}$-distance $570(P<0.0001)$ whereas for other measures only ABSI $\left(\chi^{2}\right.$-distance $551(P<0.0001))$ was close. For all-female $\left(\chi^{2}\right.$-distance $\left.147.688, P<0.0001\right)$ and all-male $\left(\chi^{2}\right.$-distance 434.372, $\left.P<0.0001\right)$ SBSI provided overall best result. Also after splitting into BMI categories, SBSI provided the best performance as well. For all-male, all-female, and all subjects SBSI outperformed ABSI in all BMI categories except underweight (See Table 2.6).

These results suggest that SBSI is the best anthropometric measure and distinguishes the Kaplan-Meier curves better than the other existing body measures tested.

Table 2.5: Results of Cox proportionality hazard modeling using different anthropometric measures.

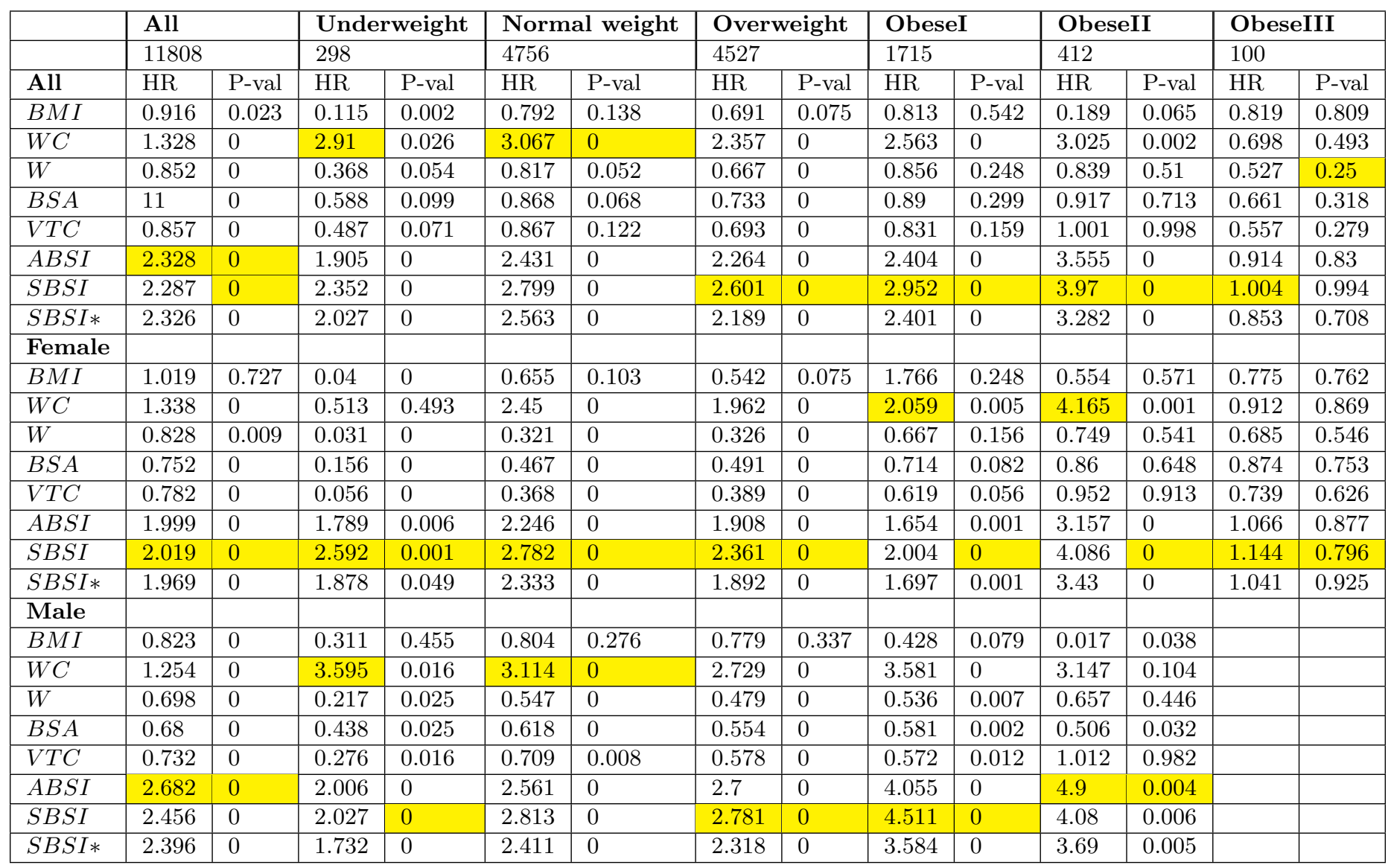

Results are shown for all subjects, females only, males only, and for different BMI categories. Results for the obese III category are not very reliable, as the number of data points were relatively low $0.85 \%, \mathrm{~N}=100$ (female 73 , male 27). Notice that the P-values for this group is generally more than 0.05 for all cases. We included the results for completeness. Highlighted cells indicate those with the best values. $S B S I^{*} \triangleq \frac{H^{2} W C}{B S A V T C}$, see section on Discussion.

From the results, ABSI and SBSI produced the overall best results using the KM curves on 
Table 2.6: Results using the log-rank test on the KM curves for different anthropometric measures.

\begin{tabular}{|c|c|c|c|c|c|c|c|c|c|c|c|c|c|c|}
\hline & \multicolumn{2}{|l|}{ All } & \multicolumn{2}{|c|}{ Underweight } & \multicolumn{2}{|c|}{ NormalWeight } & \multicolumn{2}{|c|}{ Overweight } & \multicolumn{2}{|l|}{ ObeseI } & \multicolumn{2}{|c|}{ ObeseII } & \multicolumn{2}{|c|}{ ObeseIII } \\
\hline & \multicolumn{2}{|l|}{11808} & \multicolumn{2}{|l|}{298} & \multicolumn{2}{|l|}{4756} & \multicolumn{2}{|l|}{4527} & \multicolumn{2}{|l|}{1715} & \multicolumn{2}{|l|}{412} & \multicolumn{2}{|l|}{100} \\
\hline All & $\chi^{2}$-di & $\mathrm{P}$-val & $\chi^{2}-\mathrm{di}$ & P-val & $\chi^{2}$-di & $\mathrm{P}$-val & $\chi^{2}$-di & P-val & $\chi^{2}-\mathrm{di}$ & P-val & $\chi^{2}$-di & P-val & $\chi^{2}-\mathrm{di}$ & $\mathrm{P}-\mathrm{I}$ \\
\hline$W C$ & 59.465 & 0 & 0.305 & 0.959 & 153.059 & 0 & 58.597 & 0 & 11.813 & 0.008 & 1.288 & 0.732 & 0.06 & 0.9 \\
\hline$W T$ & 12.571 & 0.006 & 0.823 & 0.844 & 5.065 & 0.167 & 21.63 & 0 & 0.499 & 0.919 & 0.618 & 0.892 & 0.03 & 0.9 \\
\hline$B S A$ & 18.531 & 0 & 0.904 & 0.825 & 1.862 & 0.602 & 19.303 & 0 & 1.206 & 0.752 & 1.273 & 0.736 & 0.61 & 0.8 \\
\hline$V T C$ & 16.994 & 0.001 & 0.229 & 0.973 & 1.814 & 0.612 & 26.066 & 0 & 1.745 & 0.627 & 0.894 & 0.827 & 3.78 & 0.2 \\
\hline$A B S I$ & 551.126 & 0 & 34.098 & 0 & 356.806 & 0 & 147.489 & 0 & 53.747 & 0 & 18.375 & 0 & 0.09 & 0.9 \\
\hline$S B S I$ & 570.045 & 0 & 11.947 & 0.008 & 296.451 & 0 & 202.795 & 0 & 130.434 & 0 & 43.603 & 0 & 1.08 & 0.7 \\
\hline$S B S I *$ & 509.009 & 0 & 22.948 & 0 & 293.144 & 0 & 128.251 & 0 & 130.434 & 0 & 27.759 & 0 & 1.35 & 0.7 \\
\hline \multicolumn{15}{|l|}{ Fem } \\
\hline$W C$ & 22.507 & 0 & 0.123 & 0.989 & 16.834 & 0.001 & 14.021 & 0.003 & 5.947 & 0.114 & 0.665 & 0.881 & 0.08 & 0.9 \\
\hline$W T$ & 6.926 & 0.074 & 0.123 & 0.989 & 10.973 & 0.012 & 29.139 & 0 & 0.545 & 0.909 & 0.2 & 0.978 & 0.08 & 0.9 \\
\hline$B S A$ & 14.943 & 0.002 & 0.42 & 0.936 & 14.599 & 0.002 & 22.985 & 0 & 2.838 & 0.417 & 0.704 & 0.872 & 0.13 & 0.9 \\
\hline$V T C$ & 11.18 & 0.011 & 0.036 & 0.998 & 16.017 & 0.001 & 20.318 & 0 & 8.485 & 0.037 & 0.162 & 0.983 & 0.04 & 0.9 \\
\hline$A B S I$ & 141.697 & 0 & 4.554 & 0.208 & 90.793 & 0 & 36.866 & 0 & 13.307 & 0.004 & 11.657 & 0.009 & 0.09 & 0.9 \\
\hline$S B S I$ & 147.688 & 0 & 4.398 & 0.222 & 105.964 & 0 & 53.344 & 0 & 22.202 & 0 & 18.08 & 0 & 0.36 & 0.9 \\
\hline$S B S I *$ & 135.758 & 0 & 5.847 & 0.119 & 81.397 & 0 & 38.768 & 0 & 14.715 & 0.002 & 18.864 & 0 & 4.25 & 0.2 \\
\hline \multicolumn{15}{|l|}{ Male } \\
\hline$W C$ & 25.961 & 0 & 0.199 & 0.978 & 108.953 & 0 & 48.633 & 0 & 10.759 & 0.013 & 0.099 & 0.992 & & \\
\hline$W T$ & 41.747 & 0 & 0.199 & 0.978 & 15.622 & 0.001 & 32.002 & 0 & 6.752 & 0.08 & 0.099 & 0.992 & & \\
\hline$B S A$ & 50.449 & 0 & 0.404 & 0.939 & 19.212 & 0 & 30.106 & 0 & 8.382 & 0.039 & 0.292 & 0.962 & & \\
\hline$V T C$ & 30.91 & 0 & 0.492 & 0.921 & 6.043 & 0.11 & 19.114 & 0 & 14.401 & 0.002 & 0.192 & 0.979 & & \\
\hline$A B S I$ & 415.644 & 0 & 23.068 & 0 & 251.83 & 0 & 130.799 & 0 & 47.054 & 0 & 9.609 & 0.022 & & \\
\hline$S B S I$ & 434.372 & 0 & 9.419 & 0.024 & 190.348 & 0 & 156.804 & 0 & 124.224 & 0 & 15.347 & 0.002 & & \\
\hline$S B S I *$ & 420.395 & 0 & 22.986 & 0 & 210.401 & 0 & 117.32 & 0 & 67.773 & 0 & 13.571 & 0.004 & & \\
\hline
\end{tabular}

Results are shown for all subjects, females only, males only, and for different BMI categories. Results for the obese III category are not very reliable, as the number of data points were relatively low $0.85 \%, \mathrm{~N}=100$ (female 73 , male 27). We can notice that the P-values for this group is generally more than 0.05 for all the cases. We included the results for completeness. Highlighted cells indicate those with the best values. $S B S I^{*} \triangleq \frac{H^{2} W C}{B S A V T C}$, see section on Discussion.

BMI categories. To further analyze the differences between ABSI and SBSI, we considered two BMI categories. Fig. 2.5 and Fig. 2.6 show the KM plots for two BMI categories (overweight, and obese I), for both ABSI and SBSI. As expected, both measures indicate that female subjects generally have better survival rates when compared with male subjects. SBSI produced an overall better performance in modeling survival over time, when compared with ABSI. 


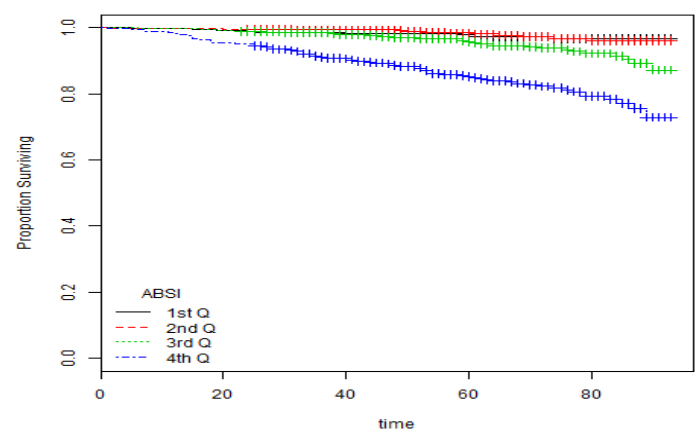

(a) male

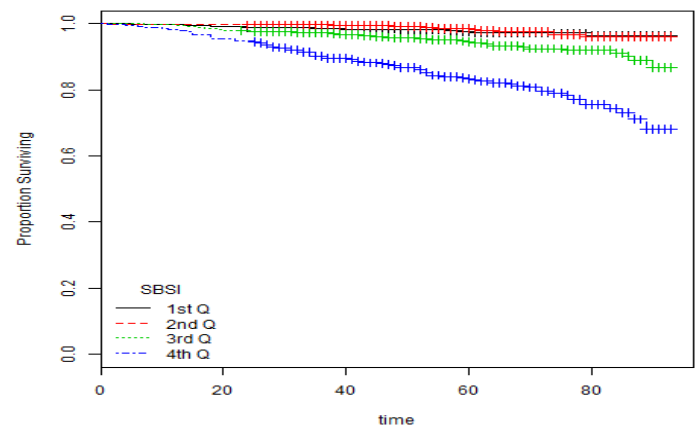

(c) male

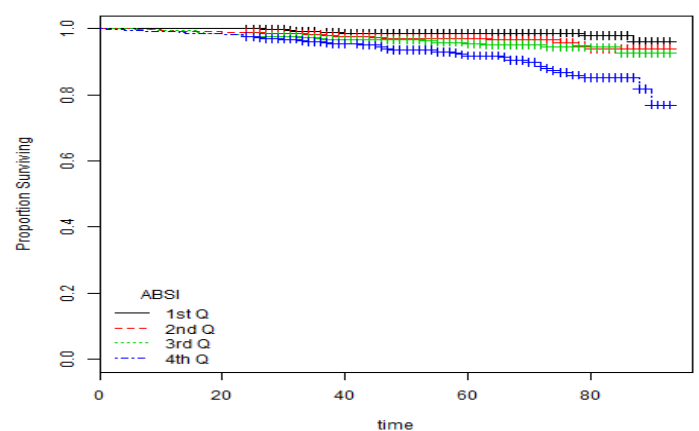

(b) female

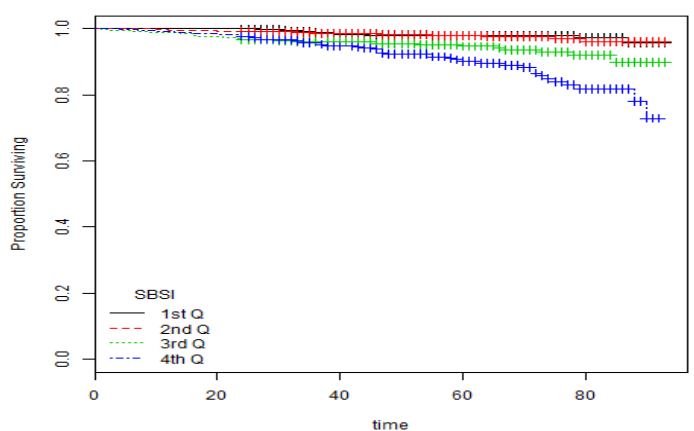

(d) female

Figure 2.5: The KM curves using ABSI and SBSI on subjects in the BMI category overweight. a: ABSI (male); b: ABSI (female); c: SBSI (male); d: SBSI (female). As expected, both measures indicate that female subjects have better survival rates when compared with male subjects. SBSI shows an overall better prediction performance than ABSI. 1st Q, 2nd Q, etc. denote respectively 1st quartile, 2nd quartile, etc.

\subsection{Discussion}

Our proposed surface-based body shape index (SBSI) is constructed based on four key anthropometric determinants of body shape and body size: body surface area (BSA), vertical trunk circumference (VTC), height (H) and waist circumference (WC). Considered at a given height, SBSI depends on WC divided by BSA and VTC. While the BSA measures the whole body, WC and VTC measure the trunk region, with WC measured horizontally, while VTC is measured vertically. More importantly, both WC and VTC are strongly associated with 


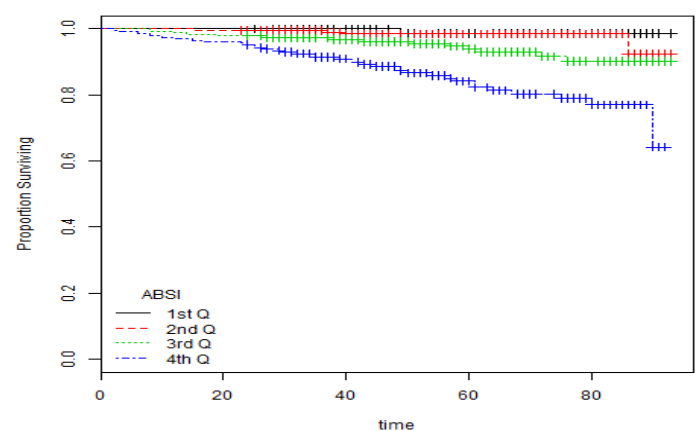

(a) male

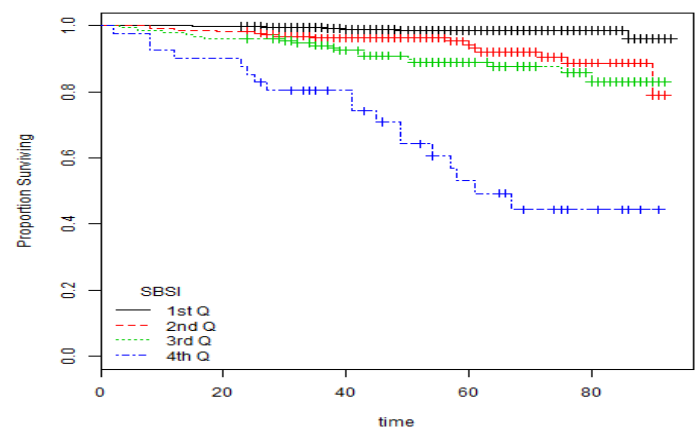

(c) male

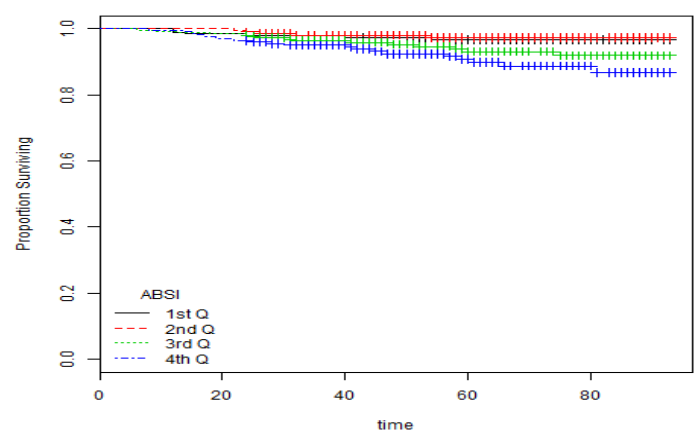

(b) female

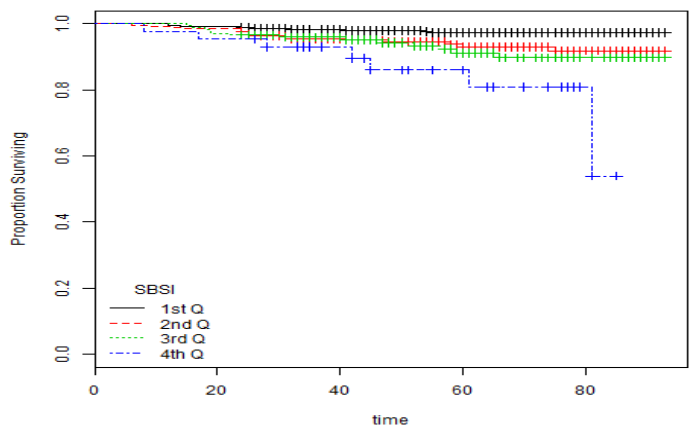

(d) female

Figure 2.6: The KM curves using ABSI and SBSI on subjects in the BMI category obese I. a: ABSI (male); b: ABSI (female); c: SBSI (male); d: SBSI (female).

abdominal fat. Previous studies [18, 26, 72, 91 show that mortality hazard is highly related to abdominal fat. Given that SBSI has a strong association with mortality hazard, and given its definition based on WC and VTC, we suspect that SBSI will also have a significant association with abdominal fat or body volume around the waist or the trunk.

Applying SBSI initially gives reasonable performance when compared with existing body shape measures. In particular, it produced a performance that is similar to that of ABSI on most cases, and better for some BMI categories. However, given the SBSI formula, it is natural to consider some variations on the definition of SBSI. For instance, one simplification would be to remove the fractional exponents on the variables, approximating them with integral exponents. Using this, we get a simpler formula (denoted SBSI*), whose values 
have no units:

$$
S B S I \triangleq \frac{H^{7 / 4} W C^{5 / 6}}{B S A V T C} \cong \frac{H^{2} W C}{B S A V T C}=S B S I^{*}
$$

Not surprisingly, this simplified formula shows very competitive performance, producing results that are generally close to the original SBSI. See the rows denoted SBSI* in the tables (Table 2.5 and Table 2.6). This competitive performance implies that SBSI* could be used in place of SBSI, depending on available computational resources, since SBSI* is just a simple unitless ratio, and easy to compute.

The measurement protocol can influence the relationship among measures for two different studies [26]. And differences between the populations involved in the study could be significant. For example, conclusions from a study based on anthropometric measurements on a Chinese population may not completely hold when applied to, say, a US population; In our work, we used two different datasets, (CAESAR and NHANES), with some differences in the way the measurements were acquired. Since we used regression parameters learned from CAESAR to apply to NHANES data, we first verified that the two data sets had similar general statistics. Participants in both studies were similar (mostly, North American, and Caucasian). The mean and standard deviation for some key attributes are as follows: height (NHANES 167.72 $\pm 10.1 \mathrm{~cm}$, CAESAR 170 $\pm 10.25 \mathrm{~cm}$ ), weight (NHANES $74 \pm 15.8 \mathrm{~kg}$, CAESAR $77 \pm 19.79 \mathrm{~kg}$ ), waist circumference (NHANES $92 \pm 13.23 \mathrm{~cm}$, CAESAR $84.77 \pm 14.43 \mathrm{~cm}$ ). Thus, at least, for these key measurements, the values from the two datasets are within one standard deviation of each other.

Age is an important factor in analyzing the mortality hazard in a population. Although the SBSI generally increased with increasing age, it was still not clear exactly how age will impact the mortality hazard modeling. To further investigate this potential connection, we categorized the study population into different age ranges: $<20$ (1638 people with mortality count 10), 20-35 (2937 people with mortality count 14), 36-50 (2501 people with 
mortality count 51), 51-70 (2678 people with mortality count 178), and > 70 (2054 people with mortality count 448). Tables 2.8 and 2.9 (Supplementary Materials) respectively, show the results for using the Cox proportionality hazard model and log-rank test on the KM curves, for mortality studies using the age categories. From the results, when using all subjects, the SBSI provided a more accurate model of the mortality hazard for people older than 35. (We ignore results for those $<20$ and $20-35$ group, since these groups do not contain enough mortality information). For cases of all, female-only, and male-only, SBSI performed very well on both hazard ratio and log-rank test, for the age categories 36 - 50, $51-70$, and $>70$. For the $51-70$ group, for all people, the hazard ratio was 1.626 . The log-rank test resulted in a $\chi^{2}$-distance of $34.058(P<0.0001)$, almost twice as the second best ABSI (17.620, $P<0.0001)$. Thus, the KM-curves for this case is expected to be more easily distinguishable for SBSI.

In this study, we have reported results on the prediction of all-cause mortality using the proposed surface-based body shape index. We applied the index on samples from the NHANES dataset (age range 18-85), using standard BMI categories. The 11808 people in our dataset were grouped as follows: 298 underweight, 4756 normal weight, 4527 overweight, 1715 obese I, 412 obese II, 100 obese III. Our results showed that the mortality hazard as measured by SBSI (and also ABSI) does not necessarily increase monotonically with BMI. For instance, the overweight category (HR 2.264, $P<0.0001$ ) showed a lower hazard ratio than the normal weight category (HR 2.799, $P<0.0001$ ). Similarly, the underweight group had a lower all-cause mortality hazard (HR 2.352, $P<0.0001)$ than the normal weight group. The hazard ratio increased for obese I category (HR 2.952, $P<0.0001)$ and obese II category (HR 3.970, $P<0.0001)$. This result is consistent with previous studies on mortality hazard and BMI categories $[76,96$. We did not observe a consistent increase in mortality for increasing BMI categories. Our results using the surface-based body shape index are also consistent with previous observations of lower mortality among slightly obese and overweight groups of people [31,88] when compared with the normal weight category. Doehner et al, [44] discussed 
this phenomenon, in terms of the "obesity paradox".

Table 2.7: Key anthropometric attributes for study participants (using the CAESAR dataset).

\begin{tabular}{|l|l|l|l|l|l|l|}
\hline & All & \multicolumn{2}{l|}{ Female } & \multicolumn{2}{l|}{ Male } \\
\hline & Average & SD & Average & SD & Average & SD \\
\hline Age $(\mathrm{y})$ & 39.621 & 12.073 & 39.821 & 12.122 & 39.396 & 12.02 \\
\hline Weight $(\mathrm{W})(\mathrm{kg})$ & 77.041 & 19.793 & 68.836 & 17.596 & 86.243 & 17.997 \\
\hline Height $(\mathrm{H})(\mathrm{cm})$ & 170.464 & 10.26 & 163.966 & 7.323 & 177.753 & 7.919 \\
\hline BMI $\left(\mathrm{kg} / \mathrm{m}^{2}\right)$ & 26.351 & 5.689 & 25.582 & 6.192 & 27.214 & 4.929 \\
\hline Leg Length $(\mathrm{cm})$ & 53.418 & 3.989 & 50.906 & 2.828 & 56.235 & 3.127 \\
\hline Arm Length $(\mathrm{cm})$ & 32.591 & 2.448 & 31.381 & 2.013 & 33.949 & 2.165 \\
\hline Arm Circumference $(\mathrm{cm})$ & 41.723 & 5.236 & 38.782 & 4.394 & 45.021 & 3.992 \\
\hline Waist Circumference $(\mathrm{cm})$ & 84.801 & 14.437 & 78.896 & 13.489 & 91.419 & 12.463 \\
\hline Thigh Circumference $(\mathrm{cm})$ & 60.731 & 6.992 & 60.628 & 7.617 & 60.846 & 6.217 \\
\hline Triceps Skinfold(cm) & 1.864 & 0.982 & 2.356 & 0.958 & 1.311 & 0.663 \\
\hline Subscapular skinfold $(\mathrm{cm})$ & 2.073 & 1.111 & 2.128 & 1.169 & 2.01 & 1.039 \\
\hline VTC $(\mathrm{cm})$ & 164.52 & 12.976 & 157.442 & 10.484 & 172.465 & 10.71 \\
\hline ABSI $\left(\mathrm{m}^{11 / 6} \mathrm{~kg}^{-2 / 3}\right)$ & 0.074 & 0.005 & 0.071 & 0.005 & 0.076 & 0.004 \\
\hline Body Surface Area(BSA)(cm2) & 18563 & 2524 & 17210 & 2025 & 20081 & 2137 \\
\hline WHtR & 0.497 & 0.077 & 0.482 & 0.082 & 0.514 & 0.067 \\
\hline SBSI & 0.106 & 0.006 & 0.105 & 0.006 & 0.108 & 0.005 \\
\hline
\end{tabular}

\subsection{Limitations of the approach.}

We identify some limitations in our study. One potential problem is the lack of control for certain demographics, for instance, smoking and non-smoking status, pregnancy, socioeconomic status, ancestry, etc. While these may be valid topics for future work, previous studies showed that adjusting for smoking as a variable does not significantly affect the results [108]. Similarly, pregnancy was not found to significantly impact the results.

The formulation of SBSI consists of VTC which is not available in the NHANES dataset. Thus, prediction parameters for VTC were estimated using the CAESAR dataset, and applied on subjects in the NHANES dataset. NHANES study consists of only US citizens, but CAESAR has subjects from the US, Canada, and Europe. Variability in the data collection protocols, and the general make-up of the subjects could be important sources of error. 
Table 2.8: Results for Cox Proportional Hazard applied to different age categories.

\begin{tabular}{|c|c|c|c|c|c|c|c|c|c|c|}
\hline & \multicolumn{2}{|l|}{$<20$} & \multicolumn{2}{|c|}{$20-35$} & \multicolumn{2}{|c|}{$36-50$} & \multicolumn{2}{|c|}{$51-70$} & \multicolumn{2}{|l|}{$70+$} \\
\hline All & HR & $\mathrm{P}$-value & HR & $\mathrm{P}$-value & HR & $\mathrm{P}$-value & HR & $\mathrm{P}$-value & HR & $\mathrm{P}$-value \\
\hline$\overline{\mathrm{BMI}}$ & 0.621 & 0.838 & 0.215 & 0.689 & 0.263 & 0.844 & 0.001 & 0.765 & 0 & 0.811 \\
\hline WC & 0.789 & 0.906 & 0.742 & 0.911 & 0.844 & 0.971 & 0.38 & 0.931 & 0.785 & 1.014 \\
\hline WT & 0.592 & 1.172 & 0.826 & 1.058 & 0.359 & 0.876 & 0.018 & 0.825 & 0.012 & 0.878 \\
\hline $\mathrm{BSA}$ & 1.321 & 0.344 & 1.298 & 0.297 & 0.91 & 0.505 & 0.867 & 0.071 & 0.927 & 0.125 \\
\hline VTC & 0.804 & 1.081 & 0.88 & 1.041 & 0.255 & 0.846 & 0.019 & 0.826 & 0.009 & 0.879 \\
\hline ABSI & 0.541 & 0.784 & 0.585 & 1.167 & 0.047 & 1.406 & 0 & 1.544 & 0 & 1.504 \\
\hline SBSI & 0.967 & 1.015 & 0.14 & 1.476 & 0.003 & 1.609 & 0 & 1.626 & 0 & 1.584 \\
\hline \multicolumn{11}{|c|}{ Female } \\
\hline BMI & 0.584 & 0.608 & 0.582 & 0.714 & 0.613 & 1.111 & 0.316 & 0.891 & 0.191 & 0.908 \\
\hline $\mathrm{WC}$ & 0.851 & 0.852 & 0.819 & 0.871 & 0.25 & 1.304 & 0.928 & 1.012 & 0.511 & 1.053 \\
\hline WT & 0.571 & 0.553 & 0.721 & 0.779 & 0.761 & 1.08 & 0.293 & 0.86 & 0.058 & 0.841 \\
\hline $\mathrm{BSA}$ & 0.653 & 0.593 & 0.881 & 0.832 & 1.042 & 0.861 & 0.867 & 0.261 & 0.845 & 0.03 \\
\hline VTC & 0.599 & 0.608 & 0.581 & 0.681 & 0.697 & 1.104 & 0.14 & 0.808 & 0.018 & 0.815 \\
\hline ABSI & 0.501 & 1.606 & 0.643 & 1.275 & 0.068 & 1.611 & 0.006 & 1.415 & 0 & 1.408 \\
\hline SBSI & 0.393 & 1.884 & 0.358 & 1.63 & 0.252 & 1.355 & 0 & 1.558 & 0 & 1.48 \\
\hline \multicolumn{11}{|l|}{ Male } \\
\hline BMI & 0.817 & 0.911 & 0.236 & 0.646 & 0.036 & 0.632 & 0 & 0.659 & 0 & 0.7 \\
\hline $\mathrm{WC}$ & 0.751 & 0.875 & 0.566 & 0.82 & 0.069 & 0.673 & 0.027 & 0.773 & 0.014 & 0.827 \\
\hline WT & 0.846 & 1.071 & 0.573 & 0.834 & 0.016 & 0.594 & 0 & 0.66 & 0 & 0.682 \\
\hline $\mathrm{BSA}$ & 1.117 & 0.737 & 0.968 & 0.91 & 0.621 & 0.014 & 0.687 & 0 & 0 & 0.719 \\
\hline VTC & 0.897 & 0.952 & 0.588 & 0.839 & 0.005 & 0.547 & 0.001 & 0.69 & 0 & 0.669 \\
\hline ABSI & 0.427 & 0.673 & 0.503 & 1.333 & 0.54 & 1.164 & 0 & 1.634 & 0 & 1.528 \\
\hline SBSI & 0.802 & 0.896 & 0.098 & 1.871 & 0.005 & 1.72 & 0 & 1.611 & 0 & 1.641 \\
\hline
\end{tabular}

Given that both datasets are collected by trained professionals [40-43] and not self-reported, differences due to the collection protocol can be assumed to be minimal. In terms of content, both the datasets are statistically similar. For the same anthropometric attribute the average measurements from the two datasets were generally within one standard deviation of each other. See Table 3.1 and Table 2.7 (Supplementary Materials). 
Table 2.9: Log-rank test results for different age category.

\begin{tabular}{|c|c|c|c|c|c|c|c|c|c|c|}
\hline & \multicolumn{2}{|l|}{$<20$} & \multicolumn{2}{|l|}{$20-35$} & \multicolumn{2}{|l|}{$36-50$} & \multicolumn{2}{|l|}{$51-70$} & \multicolumn{2}{|l|}{$70+$} \\
\hline All & $\chi^{2}$-dist & $\mathrm{P}$-value & $\chi^{2}$-dist & $\mathrm{P}$-value & $\chi^{2}$-dist & $\mathrm{P}$-value & $\chi^{2}$-dist & $\mathrm{P}$-value & $\chi^{2}$-dist & $\mathrm{P}$-value \\
\hline$W C$ & 3.633 & 0.304 & 3.742 & 0.291 & 2.229 & 0.526 & 9.099 & 0.028 & 1.215 & 0.749 \\
\hline$W T$ & 0.333 & 0.954 & 6.729 & 0.081 & 1.241 & 0.743 & 10.108 & 0.018 & 5.466 & 0.141 \\
\hline$B S A$ & 1.558 & 0.669 & 4.152 & 0.246 & 1.2 & 0.753 & 4.618 & 0.202 & 1.817 & 0.611 \\
\hline$V T C$ & 0.67 & 0.88 & 7.522 & 0.057 & 1.36 & 0.715 & 11.625 & 0.009 & 8.555 & 0.036 \\
\hline$A B S I$ & 2.23 & 0.526 & 0.473 & 0.925 & 9.701 & 0.021 & 17.62 & 0.001 & 43.016 & 0 \\
\hline$S B S I$ & 0.419 & 0.936 & 2.371 & 0.499 & 7.957 & 0.047 & 34.058 & 0 & 72.415 & 0 \\
\hline \multicolumn{11}{|l|}{ Female } \\
\hline$W C$ & 2.608 & 0.456 & 3.882 & 0.275 & 2.155 & 0.541 & 4.156 & 0.245 & 2.443 & 0.486 \\
\hline$W T$ & 1.087 & 0.78 & 1.029 & 0.794 & 1.249 & 0.741 & 1.537 & 0.674 & 5.169 & 0.16 \\
\hline$B S A$ & 2.308 & 0.511 & 1.01 & 0.799 & 3.869 & 0.276 & 1.93 & 0.587 & 5.574 & 0.134 \\
\hline$V T C$ & 1.002 & 0.801 & 3.36 & 0.339 & 0.951 & 0.813 & 6.921 & 0.074 & 5.524 & 0.137 \\
\hline$A B S I$ & 5.074 & 0.166 & 0.981 & 0.806 & 4.353 & 0.226 & 7.948 & 0.047 & 15.671 & 0.001 \\
\hline$S B S I$ & 2.973 & 0.396 & 3.778 & 0.286 & 0.753 & 0.861 & 13.878 & 0.003 & 25.31 & 0 \\
\hline \multicolumn{11}{|l|}{ Male } \\
\hline$W C$ & 5.564 & 0.135 & 3.47 & 0.325 & 6.139 & 0.105 & 29.56 & 0 & 4.62 & 0.202 \\
\hline$W T$ & 3.019 & 0.389 & 1.69 & 0.639 & 10.413 & 0.015 & 26.433 & 0 & 22.36 & 0 \\
\hline$B S A$ & 2.672 & 0.445 & 3.002 & 0.391 & 5.759 & 0.124 & 14.895 & 0.002 & 15.869 & 0.001 \\
\hline$V T C$ & 1.952 & 0.582 & 1.831 & 0.608 & 12.047 & 0.007 & 16.346 & 0.001 & 21.869 & 0 \\
\hline$A B S I$ & 0.868 & 0.833 & 0.646 & 0.886 & 7.371 & 0.061 & 8.086 & 0.044 & 18.652 & 0 \\
\hline$S B S I$ & 2.924 & 0.404 & 3.005 & 0.391 & 9.53 & 0.023 & 18.186 & 0 & 34.137 & 0 \\
\hline
\end{tabular}




\section{Chapter 3}

\section{Estimating Adult Age from Human Body Shape}

\subsection{Introduction}

Age estimation is an important problem that has witnessed an increased attention, given its role in various daily activities, from health assessment [94, to social interaction [103], to forensic science [64,145], to security and identity profiling [57]. Although age estimation has been practiced for centuries, accurate age estimation is known to be a very difficult problem. Doing this automatically by a machine is an even much more onerous task [57,65]. The major challenge is that most measures used to characterize age, for instance, visual appearance, and biological/physiological markers vary significantly from person to person, even for people with the same chronological age. The reason is that many unknowns (e.g., genetics 48, 145, nutrition [125], body shape [118], health condition [125], social conditions [71], life style [36], weather [13], and even cultural considerations [13]), all contribute to influence the perceived age of an individual.

The process of aging is complex and affects all biological systems. Age has a deep connection with health and mortality [118]. In general, a younger person is expected to have a better health condition and his/her mortality hazard should be low in comparison with a relatively older person. But two different people of the same chronological age may have very different 
health conditions and mortality hazards. This brings up an important classification of age namely, chronological age versus biological age. Chronological age is based on the date of birth. However, biological age is a conceptual idea that a person's true age can be different from his/her chronological age. Although biological age is a loosely used concept and lacks precise definition, it is often viewed as the true age of an individual [83]. Thus, biological age provides a better measure of the life expectancy of an individual than his or her chronological age. The common idea is to calculate biological age based on some age-dependent variables [17, 35, 74, 127], where chronological age may or may not be a required variable depending on the application. Chronological age, with its dependence on the true birth date, is supposed to be simple, especially when the genuine birth certificate is available. There are, however, various scenarios or applications when the chronological age is required, but the true birth date is unknown, and a genuine birth certificate may not be available. Examples here include in forensic and crime investigations [64, 121, 145], biometrics [53, 56, 65] for border control and security, competitive sports and age-based competitions, and in immunization and vaccination (even for adults [7], for instance, in a refugee camp). In this work, our focus is on chronological age (more detailed treatment on biological age can be found in various related publications 21, 49, 127]). In particular, we investigate the question of whether human anthropometric measurements can be used for reliable estimation of chronological age in adults.

The human body grows and matures with age, especially in children and adolescents. Therefore, the basic approach to age prediction is to compare measurements of physical maturity of the body with age. However, due to individual variations in the timing of skeletal and dental development, the results of any method will be subject to uncertainty when applied to a single individual. Moreover, age prediction is difficult because people in different subgroups age at different rates. The study of aging differences across subgroups necessarily involves the characteristics of people along with their chronological age. Bones are known to mature at fairly standard rates, while growing through childhood [86]. Like normal human 
growth, bones stop growing around ages 17 to 25 . A young person who is still growing have special growth plates in his/her bones, but at about age 20, the bones stop growing [100]. Clinical growth charts showing the expected growth at a given age are available for both boys and girls (http://www.cdc.gov/growthcharts/clinical_charts.htm). These charts are easily found in pediatric clinics and hospitals, and are often used in routine growth monitoring, and in assessment of healthy body development in children and adolescents. For a child having a normal growth, the charts suggest that we can predict his/her age using height and weight. A popular approach for estimating the age from todlers to adolescent is the use of dental features [142], e.g., by checking the types of teeth visible above the gum line. Age for children and adolescents can also be estimated via microscopic examination of the enamel [147], or by the use of dental radiographs [93].

For adults however, the accuracy of these methods is significantly reduced [121]. Given their dependence on growth, most of these methods used to predict chronological age in children and adolescents do not work for adults. For instance, adult height remains fairly constant until around the seventies, when a decrease with age may be observed. Not surprisingly, various other approaches have been proposed for age estimation for adults, from semi-automated to fully automated methods. The methods range from image-based techniques [38, 53, 57, 65 to those that use biomarkers [114, 127], to those that exploit genetic information [67, 137]. Age estimation from face is perhaps the most popular [65] of the image-based approaches. These have generally used biometric features (both hard and soft biometrics) based on holistic or local face features (face-shape, skin texture, areas of eye, nose, forehead, etc.). It remains a challenging problem, because face aging is a complex process and involves many factors, e.g., genetic, expression, food habit, lifestyle, psychological disposition, location or environment where a person lives in, etc. Different approaches have been employed, for example, active appearance based methods (mean absolute error (MAE) 3.29 years for 4 age classes, see Xia et al [143]), appearance based approaches that consider texture, shape, and biologicallyinspired features (MAE 2.6, Guo et al [61]), approaches based on aging pattern subspace 
extracted from a sequence of images that represents chronological advancement or pattern of aging (MAE 6.77 years [57]), automated age kernel partial least square regression (KPLS) using discriminant features (MAE $=4.18$ using KPLS, MAE=5.09 using support vector regression) [60]. Detailed surveys of methods for face-based age estimation can be found in $\mathrm{Fu}$ et al [53], and Han et al [65]. We note that while other methods such as body measurements are relatively successful in predicting chronological age for children and adolescents, these age categories pose the most difficult challenge for face-based methods [119].

Putin et al [114] studied the use of biomarkers in a deep learning framework for chronological age prediction. In this work they utilized an ensemble of multiple deep neural networks (DNN) and trained on blood biochemistry samples. They employed a variation of the implementation of permutation feature importance (PFI) 16 technique to evaluate the relative importance of each blood biochemistry marker to ensemble accuracy. The best performance by a DNN was MAE of 6.07 years in predicting chronological age and the ensemble learning produced MAE of 5.55 years. They identfied the 5 most important biomarkers for predicting human chronological age: albumin, glucose, alkaline phosphatase, urea and erythrocytes. Also age prediction from DNA [137] is of interest. Major approaches to DNA-based age estimation include the free radical [67], mitochondrial [120], and telomere 28,29] based methods. Eipel et al [48] used DNA methylation changes to estimate chronological age. They trained an age predictor based on three age-associated CpG island sites (PDE4C, ASPA, and ITGA2B) for swab samples and found mean absolute deviation of 7.03 years in validation set. Bocklandt et al [24] showed that methylation of the genes EDARADD, TOM1L1, and NPTX2 is associated with age, and by applying regression they achieved a prediction accuracy of 5.2 years.

Each existing method has its advantages and weaknesses. Bone and dental based approaches are not very accurate for ages above 25. Similarly for methods based on body height and weight, although use of growth charts is a good starting point, this approach may not work for a child whose weight and height deviates from the average values. Despite the intensive 
work on face-based age estimation, various factors based on imaging make age estimation from face still very difficult. Also a major consideration is the range of age that is being estimated. Estimating adult age for humans still remains a difficult challenge.

In this work, we consider the use of human body shape (as described by whole-body anthropometric measurements) for reliable estimation of adult human age. We propose a multistage framework to estimate adult age by first performing body shape modeling, and then clustering of body shapes based on their shape model. We then construct models to learn the appropriate estimation parameters for a specified body shape cluster and a predicted probable age group. The last stage uses the constructed model to make a final estimate of the age.

\subsection{Methodology}

\subsubsection{Dataset}

We used anthropometric data from the National Health and Human Nutrition Examination Surveys (NHANES) 1999 - 2004 [2]. NHANES uses a complex cluster design to sample members of the civilian USA population who are not institutionalized. NHANES uses stratified multistage probability to sample the data. Ethnicity included white, black, Mexican and others. Anthropometric measurements included BMI, height, weight, and waist circumference. We used the NHANES mobile examination center sample. The mobile examination center used trained examiners who followed standardized protocols to measure the anthropometric parameters. We started with 31126 individuals. After refining, we obtained 11,808 individuals aged 18 to 85 years, with 701 deaths during the $2-8$ years of follow-up (19992006). Fig. S1 shows the refinement steps. Table 3.1 shows the key anthropometric attributes used in this study. ABSI (A Body Shape Index) was computed as defined by Krakauer and Krakauer [85], while SBSI (Surface-based Body Shape Index) was computed following Rahman and Adjeroh [118]. Vertical Trunk Circumference (VTC) for the subjects was estimated 
for NHANES dataset using the approach described by Rahman and Adjeroh [118].

We also used data from the Civilian American and European Surface Anthropometry Resource (CAESAR) 122] dataset (http://store.sae.org/caesar/) which includes manual hand measurements of the various anthropometric attributes, recorded as both 3D and 1D data. For our purpose, we used the 1D datasets from the CAESAR survey, which contains 2400 US and Canadian civilians, aged 18-65. We selected key human body measurements, as reported by Adjeroh et al [12] and Cao et al [30]. From our analysis, the key measurements shared by both datasets tend to have similar general statistics. For example, the mean and standard deviation were observed as follows: height (NHANES $167.7 \pm 10.1$; CAESAR $170.5 \pm 10.2$ ), waist circumference (NHANES $92.2 \pm 13.2$; CAESAR $84.8 \pm 14.4$ ), weight (NHANES $74 \pm$ 15.8; CAESAR $77 \pm$ 19.8), BMI (NHANES $26.2 \pm 4.7$; CAESAR $26.3 \pm$ 5.7). The age distributions for both CAESAR and NHANES datasets are shown in Fig. S2. Table 3.1 shows the key anthropometric measurements used in this study.

\subsubsection{Data Availability}

The datasets generated during and/or analysed during the current study are available from the corresponding author upon request.

\subsubsection{Key Observations and Motivation}

Although body measurements can predict age for children and adolescents to a reasonable accuracy, using these to estimate age for adults is still a very challenging task. The question of whether anthropometric measurements can indeed predict age for adult humans has not been well studied []. Motivated by new observations based on some recently proposed human body shape indices, this paper investigates the question of reliability of human anthropometric measures in estimating chronological age in adults. Table 3.2 shows the correlation between age and some popular anthropometric body indices. The table shows the correlation using 
Table 3.1: Anthropometric attributes for study participants using the NHANES dataset.

\begin{tabular}{|c|c|c|c|}
\hline & All $(\mathrm{N}=11808)$ & Female $(\mathrm{N}=5840)$ & Male $(\mathrm{N}=5968)$ \\
\hline Attribute & Average \pm SD & Average \pm SD & Average \pm SD \\
\hline Age(years) & $45.69 \pm 20.73$ & $45.42 \pm 20.92$ & $45.96 \pm 20.53$ \\
\hline Weight(W)(kg) & $74.02 \pm 15.80$ & $67.91 \pm 14.07$ & $79.99 \pm 15.11$ \\
\hline $\operatorname{Height}(\mathrm{H})(\mathrm{cm})$ & $167.73 \pm 10.10$ & $160.94 \pm 7.14$ & $174.37 \pm 7.93$ \\
\hline $\operatorname{BMI}\left(k g / m^{2}\right)$ & $26.23 \pm 4.7$ & $26.20 \pm 5.07$ & $26.25 \pm 4.31$ \\
\hline Leg Length(LEG) $(\mathrm{cm})$ & $40.56 \pm 3.79$ & $38.64 \pm 3.22$ & $42.44 \pm 3.34$ \\
\hline Arm Length $(\mathrm{ARM})(\mathrm{cm})$ & $37.09 \pm 2.73$ & $35.49 \pm 2.14$ & $38.65 \pm 2.31$ \\
\hline Arm Circumference(ARMC) $(\mathrm{cm})$ & $31.26 \pm 4.07$ & $30.08 \pm 4.10$ & $32.42 \pm 3.69$ \\
\hline Waist Circumference(WC) $(\mathrm{cm})$ & $92.19 \pm 13.23$ & $89.57 \pm 13.04$ & $94.75 \pm 12.91$ \\
\hline Thigh Circumference(THIC) $(\mathrm{cm})$ & $51.36 \pm 5.98$ & $50.92 \pm 6.35$ & $51.79 \pm 5.57$ \\
\hline Triceps Skinfold(TRI)(cm) & $17.68 \pm 8.07$ & $22.39 \pm 7.24$ & $13.06 \pm 5.88$ \\
\hline Subscapular skinfold(SUB) $(\mathrm{cm})$ & $19.07 \pm 7.57$ & $20.05 \pm 7.85$ & $18.12 \pm 7.15$ \\
\hline Vertical Trunk Circumference(VTC)(cm) & $174.92 \pm 11.91$ & $170.35 \pm 10.85$ & $179.40 \pm 11.18$ \\
\hline A Body Shape Index(ABSI $)\left(m^{11 / 6} k^{-2 / 3}\right)$ & $0.08 \pm 0.01$ & $0.08 \pm 0.01$ & $0.08 \pm 0.01$ \\
\hline Body Surface Area(BSA) $\left(\mathrm{cm}^{2}\right)$ & $18074 \pm 2168$ & $16927 \pm 1759$ & $19196 \pm 1930$ \\
\hline Waist to Height Ratio (WHtR) & $0.55 \pm 0.08$ & $0.56 \pm 0.08$ & $0.54 \pm 0.08$ \\
\hline Surface Based Body Shape(SBSI) & $0.11 \pm 0.01$ & $0.11 \pm 0.01$ & $0.11 \pm 0.01$ \\
\hline VTC to WC Ratio (VTWR) & $1.92 \pm 0.18$ & $1.93 \pm 0.19$ & $1.92 \pm 0.18$ \\
\hline Neck Circumference $(\mathrm{NC})(\mathrm{cm})$ & $34.63 \pm 3.68$ & $32.54 \pm 3.29$ & $36.68 \pm 2.77$ \\
\hline BSA to VTC to WC Ratio (BSVTR) & $103.01 \pm 6.13$ & $99.15 \pm 4.74$ & $106.78 \pm 4.85$ \\
\hline VTC to NC Ratio (VTNR) & $5.12 \pm 0.77$ & $5.31 \pm 0.84$ & $4.93 \pm 0.65$ \\
\hline VTC to Height Ratio (VTHR) & $1.04 \pm 0.06$ & $1.06 \pm 0.06$ & $1.03 \pm 0.05$ \\
\hline
\end{tabular}


direct body measurements for both Pearson's $\rho$ (upper half), and Kendall's $\tau$ (lower half). We can observe from Table 3.2 that age has a good positive correlation with ABSI and SBSI and reasonable correlation with Waist Circumference (WC), and a reasonable negative correlation with Leg Length, and Thigh Circumference (THIC). Age has relatively low correlation with BMI and weight. However, there exists better correlation between some other body measures with age. Thus, it might be possible to estimate age based on certain anthropometric measurements. In particular, we note that, the three measures that are most correlated with age are ratio-based measures, with two of these being recently proposed body shape indices, namely ABSI (Krakauer \& Krakauer, 2012 [85]) and SBSI (Rahman \& Adjeroh, 2015 [118]).

Table 3.2: Correlation coefficient between anthropometric measurements: Pearson's $\rho$ (upper half), and Kendall's $\tau$ (lower half).

\begin{tabular}{|c|c|c|c|c|c|c|c|c|c|c|c|c|c|c|c|c|}
\hline & $\mathbf{W}$ & $\mathbf{H}$ & BMI & LEG & ARM & ARMC & WC & THIC & TRI & SUB & VTC & ABSI & BSA & WHtR & SBSI & AGE \\
\hline Weight(W) & 1 & 0.55 & 0.82 & 0.4 & 0.63 & 0.87 & 0.83 & 0.79 & 0.25 & 0.54 & 0.98 & 0.08 & 0.96 & 0.59 & -0.41 & 0.05 \\
\hline Height $(\mathrm{H})$ & 0.38 & 1 & -0.02 & 0.77 & 0.78 & 0.31 & 0.19 & 0.27 & -0.34 & -0.09 & 0.57 & -0.01 & 0.76 & -0.23 & -0.03 & -0.15 \\
\hline BMI & 0.61 & 0.00 & 1 & -0.04 & 0.22 & 0.83 & 0.86 & 0.77 & 0.54 & 0.7 & 0.78 & 0.09 & 0.63 & 0.86 & -0.48 & 0.16 \\
\hline LEG & 0.28 & 0.57 & -0.01 & 1 & 0.63 & 0.22 & 0.06 & 0.34 & -0.24 & -0.07 & 0.36 & -0.16 & 0.57 & -0.27 & -0.08 & -0.28 \\
\hline ARM & 0.46 & 0.59 & 0.16 & 0.45 & 1 & 0.46 & 0.39 & 0.34 & -0.18 & 0.09 & 0.62 & 0.07 & 0.75 & 0.06 & -0.06 & 0.06 \\
\hline ARMC & 0.68 & 0.21 & 0.64 & 0.16 & 0.32 & 1 & 0.73 & 0.77 & 0.36 & 0.58 & 0.85 & -0.03 & 0.78 & 0.6 & -0.53 & 0.05 \\
\hline WC & 0.62 & 0.13 & 0.67 & 0.05 & 0.26 & 0.53 & 1 & 0.56 & 0.36 & 0.62 & 0.84 & 0.54 & 0.71 & 0.91 & -0.02 & 0.34 \\
\hline THIC & 0.58 & 0.19 & 0.55 & 0.24 & 0.24 & 0.56 & 0.37 & 1 & 0.43 & 0.5 & 0.73 & -0.27 & 0.7 & 0.44 & -0.66 & -0.23 \\
\hline TRI & 0.14 & -0.25 & 0.36 & -0.18 & -0.14 & 0.21 & 0.24 & 0.27 & 1 & 0.63 & 0.3 & -0.01 & 0.08 & 0.5 & -0.38 & 0.05 \\
\hline SUB & 0.37 & -0.06 & 0.53 & -0.04 & 0.06 & 0.41 & 0.45 & 0.34 & 0.44 & 1 & 0.63 & 0.14 & 0.39 & 0.65 & -0.4 & 0.09 \\
\hline VTC & 0.86 & 0.39 & 0.58 & 0.25 & 0.44 & 0.66 & 0.63 & 0.52 & 0.18 & 0.44 & 1 & 0.16 & 0.95 & 0.59 & -0.36 & 0.07 \\
\hline ABSI & 0.07 & 0.01 & 0.09 & -0.10 & 0.06 & 0.00 & 0.39 & -0.17 & 0.01 & 0.11 & 0.13 & 1 & 0.06 & 0.54 & 0.8 & 0.52 \\
\hline BSA & 0.82 & 0.56 & 0.43 & 0.40 & 0.56 & 0.58 & 0.50 & 0.50 & 0.03 & 0.26 & 0.79 & 0.06 & 1 & 0.38 & -0.32 & -0.01 \\
\hline WHtR & 0.40 & -0.14 & 0.67 & -0.16 & 0.05 & 0.42 & 0.74 & 0.28 & 0.34 & 0.48 & 0.41 & 0.39 & 0.25 & 1 & -0.01 & 0.4 \\
\hline SBSI & -0.25 & -0.01 & -0.29 & -0.06 & -0.03 & -0.34 & 0.00 & -0.46 & -0.23 & -0.25 & -0.22 & 0.58 & -0.20 & 0.01 & 1 & 0.37 \\
\hline AGE & 0.06 & -0.09 & 0.13 & -0.18 & 0.04 & 0.05 & 0.25 & -0.14 & 0.04 & 0.08 & 0.07 & 0.37 & 0.01 & 0.29 & 0.25 & 1 \\
\hline
\end{tabular}

Fig. 3.1 shows how four anthropometric measurements vary with age, for both male and female. Subjects in our NHANES dataset had ages in the range 18-85. Mean SBSI increases consistently for male subjects but the rate of increase varies for different segments of the age range. However, for female subjects, the mean goes up from about ages 18 to 24 then goes down till about age 30; between ages 30 and 40, the mean SBSI did not have a definite pattern for females, going up and then down. After this point, the SBSI generally increased with age. Similar observations can be made for ABSI; for both male and female. For WC, ABSI and SBSI, the mean values for males were clearly separated from those for females, with the value for males being generally greater. For BMI, this distinction was not as clear. Unlike ABSI and SBSI that had a generally linear relationship with age, BMI and 


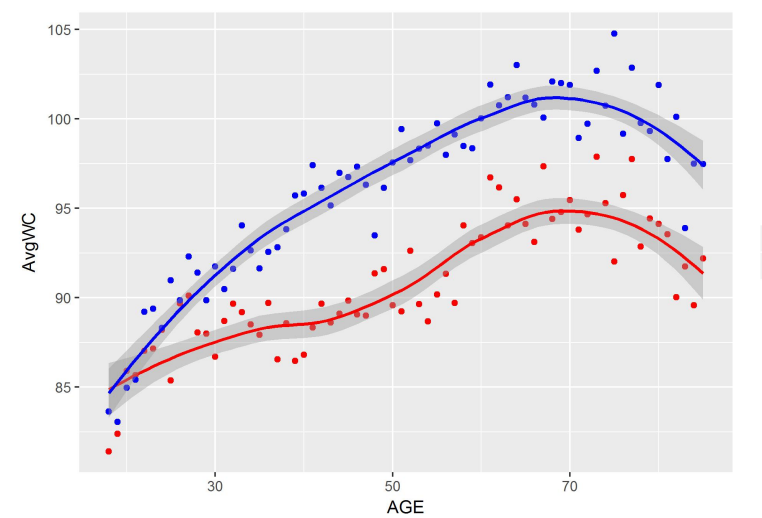

(a) WC

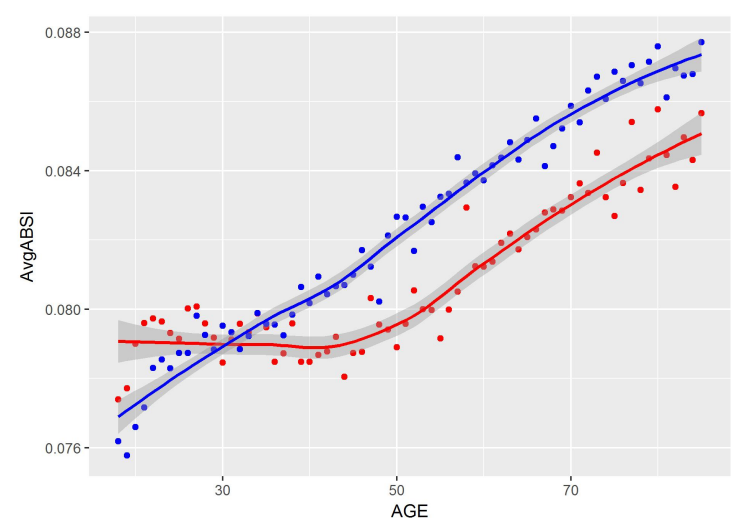

(c) ABSI

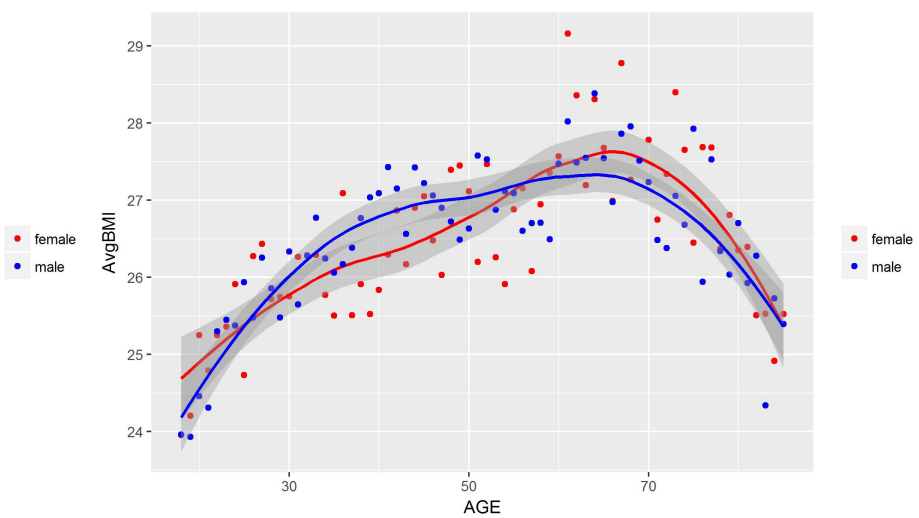

(b) BMI

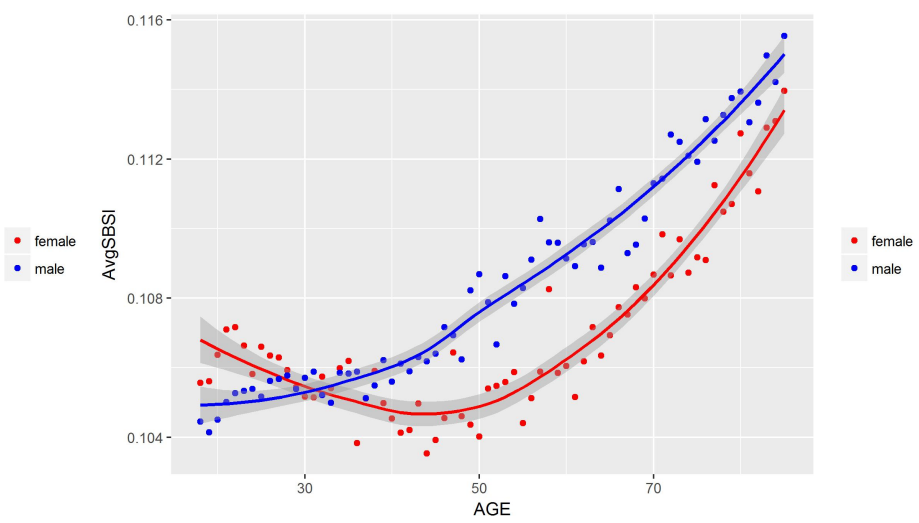

(d) SBSI

Figure 3.1: Variation of different body shape indices with age.

WC had an inverted U-like shape, with the turning points around age 75 for WC, and age 70 for BMI. These observations suggest the following: (1) Age prediction using attributes such as ABSI or SBSI will be most challenging for those in the age range between 20 and 40. (2) An appropriate age prediction method should treat males and females differently, if anthropometric attributes are being used. (3) Even within a given gender, a segmental approach using a different prediction model (or different model parameters) for different age ranges, may be required for accurate prediction. 


\subsubsection{Simple Prediction}

To appreciate the challenge of adult age estimation using body measurements, we attempted a simple experiment to estimate the ages for people in the NHANES dataset based on the anthropometric features. Initially, we considered all subjects (11808) in the dataset, and estimated their age using different regression schemes, namely Linear Regression (LR), Support Vector Machine (SVM), and Random Forest Regression (RF) applying 10-fold cross validation.

We obtained best results using SVM, with correlation coefficient $(\rho)$ of 0.72 , and mean absolute error (MAE) of 11.31. The very high MAE between the predicted and the true ages, independent of the regression method documents the high level of difficulty in this problem. Given the observations in Fig. 3.1, especially on the segmented nature of the relationship between the new bodyshape indices, (such as ABSI and SBSI) and age, a possible approach could be to perform the predictions differently for different groups, for instance, based on age group, or gender.

Thus we divided the dataset based on gender (female and male) and applied the regression algorithms. Expectedly, for male the performance improved ( $\rho=0.79, M A E=9.67$ using SVM), but females were more difficult $(\rho=0.63, M A E=13.09)$. To further test the intuition on a segmental approach to age estimation, we suppose that we already knew the age group for the subjects under consideration, and then performed the prediction separately for each age group. Hence, we divide the dataset into 6 age groups as : $G_{1}(\leq 25), G_{2}(26-$ $35), G_{3}(36-45), G_{4}(36-45), G_{5}(56-70), G_{6}(71-85)$, and then built age estimation models for each group. The results showed a significant improvement in estimation performance for each age group, with a reduced average MAE of 3.0 years over the groups. (Detailed results are presented in Supplementary Material Table S1). 


\subsubsection{Multi-stage Age Estimation}

The reduced MAE when using known age groups suggests an idea to improve the estimation

process. In practice, however, we may neither know the age (our original question to start with), nor the age group of the individual. Yet, the foregoing discussion shows that some knowledge of the age group or approximate age could help to improve the estimation results. This motivates a multi-stage age estimation framework, whereby we first estimate the age group, and then for each age group, we construct learning models suited for the body shape types found at that age group. The goal is to investigate whether anthropometric measures or combination of measures can lead to improved age estimation. While the above simple age estimation is a good first step, the estimation error is still very high. In this paper, we present a framework for automatic age estimation using anthropometric body measurements. Fig. 3.2 shows the general structure of the proposed framework. Below we describe each component of the multi-stage framework.

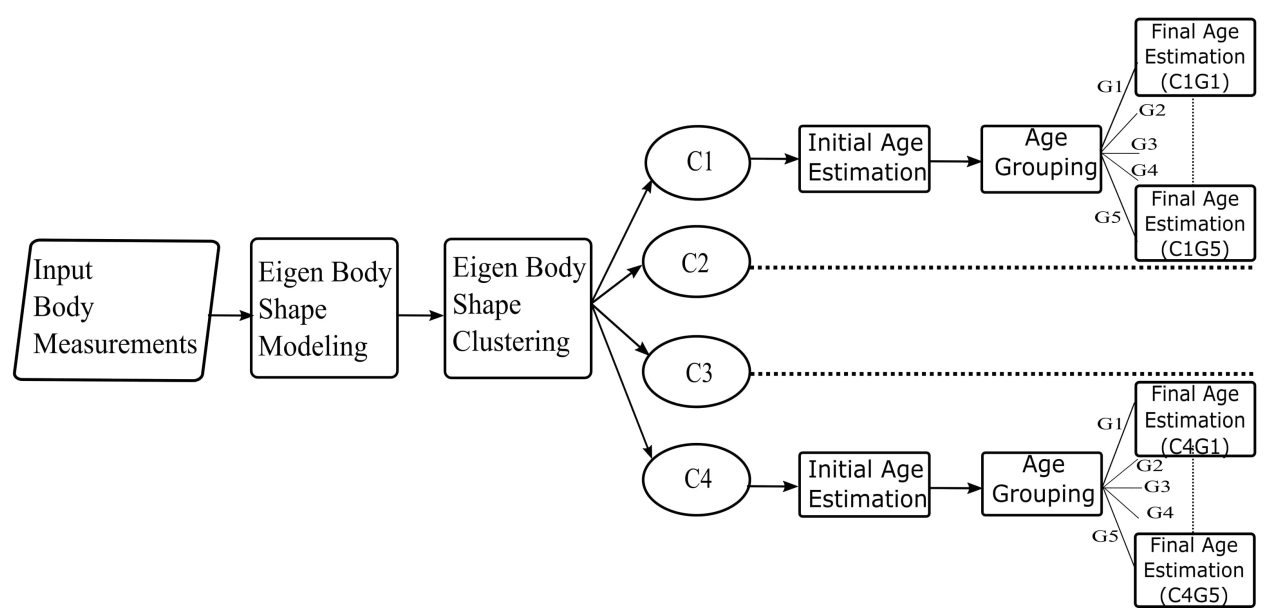

Figure 3.2: Proposed framework for multi-stage age estimation using human body measurements. 


\section{Eigen Body Shape Modeling}

The first step in the proposed approach is eigen body shape modeling. As described above, using the anthropometric measurements does not directly lead to accurate age prediction. Given that the newly introduced body attributes that are best correlated with age (ABSI and SBSI) are body shape indices, to improve the prediction ability, we first model the body shape for each individual under consideration. For this modeling, we use spectral features obtained after spectral transformation of the original measurements. We call this eigen body shape modeling, since the body shape for each subject is now described in terms of the eigen features obtained from the spectral transformation. In this work, we consider eigen body shape modeling using Principal Component Analysis (PCA) [77]. We describe this briefly under Supplementary Material.

\section{Body-Shape Clustering}

Now that we have extracted the eigen body shape features from the body measurements, the next step is to group the subjects based on their shape features. The goal is to cluster the subjects in our dataset such that each cluster contains individuals with similar body shapes as captured by the eigen body shape model. For this work, we apply simple partition clustering rather than hierarchical clustering (this is intuitive because a person belongs to a cluster based on his/her body shape features and also the clusters are non-overlapping). The $k$-means algorithm is a simple and elegant clustering method for partitioning a data set into $k$ distinct groups. It is an unsupervised learning method and performs reasonably well with large data sets. More importantly, the $k$-means algorithm tries to minimize the within-cluster variation while maximizing the between cluster variance. Thus, for body shape clustering, we applied the $k$-means algorithm on the eigen body shape features. For this work, we

empirically set $k$ to be 4 based on the results of different clustering performance indices(see Figure 3.3). 


\section{Probable Age Groups and Age Estimation}

Once we have the body shape clusters, we are now in a position to perform the first level of age estimation. For each individual in a given cluster, we use $Y$ to denote the actual/original age. Then we compute $Y^{\prime}$, our initial guess on the person's age. For this first level estimation, we use support vector regression with model parameters for the specific eigen body shape cluster. Based on the estimated age we divide each body shape cluster into 6 probable age groups, namely, $G_{1}(\leq 25), G_{2}(26-35), G_{3}(36-45), G_{4}(46-55), G_{5}(56-70), G_{6}(70+)$. We call these probable age groups since the grouping is based on the first age estimate, which may not be very accurate. Our stage of final age estimation is performed in two steps, namely, (1) second-level age estimation, and (2) refinement of age estimates using neighborhood contexts. For the first step we apply the earlier mentioned regression schemes (LR, SVM, and RF) for each age group to estimate age for each individual. We use $\tilde{Y}$ to denote the outcome at this second level of age prediction. Algorithm 1 shows the pseudo-code summarizing the proposed approach.

\subsubsection{Incorporating Neighborhood Contexts}

To further improve the estimation results, we consider the neighboring context around a given subject. Given the age grouping and the body shape clustering, each subject will be placed into one of the $|C| \times|G|$ non overlapping groups based on their predicted age and body shape model. Here $|G|$ is the number of age groups and $|C|$ is the number of body shape clusters, essentially, $|C|=k$. Because the groups are non-overlapping, the results may be unstable, especially for subjects that are close to the boundary of their group. For instance, using the age grouping scheme above, a person that is say 35 is in the same age group with some one that is 26 , while a subject that is 36 will be placed in a different group. This is a general problem with discrete grouping. For such boundary cases, second level age estimation for two people with very close ages (as estimated by the first level predictor) 





might be performed with very different models, even if they belong to the same body shape cluster. This could lead to potential instability in the results, with the final outcome being heavily dependent on possible errors during the preliminary first level estimation. To reduce this problem, the final age is computed as a weighted combination of the age predictions for the current position and its neighboring contexts.

To estimate the age for an individual in a given shape cluster, we consider this person as a point in a $|C| \times|G|$ table, based on the subject's probable age group, and shape cluster. Consider person $P$ belonging to shape cluster $i$ and age group $j$, where $i=1,2, \ldots|C|$, and $j=1,2, \ldots|G|$. We define this person's neighborhood context as the current shape cluster and age group, and the shape clusters in the nearby age group(s). That is, the neighborhood context is defined based on information from three age groups: groups $j-1, j$, and $j+1$. For person $P$, we denote the age estimated from Algorithm 1 as $\tilde{Y}_{P}$. First, we obtain $\bar{G}_{P}$, the centroid of person $P$ 's current age group, given as $\bar{G}_{P}=\frac{1}{\left|S_{P}\right|} \sum_{u \in S_{P}} \tilde{Y}_{u}$, with $S_{P}=\left\{q \mid q \in G_{j} ; P \in G_{j} ; q \neq P\right\}$, based on the probable age groups estimated using the first level age estimation $Y_{P}^{\prime}$. Next, using Algorithm 1 , we compute $\tilde{Y}_{P}^{i, j-1}$ and $\tilde{Y}_{P}^{i, j+1}$, the respective estimated ages for person $P$ using the prediction models for the left and right age groups surrounding person $P$ 's age group, $j$. Now we compute the distances: $\Delta_{i, j-1}=$ $\left|\bar{G}_{P}-\tilde{Y}_{P}^{i, j-1}\right|$ and $\Delta_{i, j+1}=\left|\bar{G}_{P}-\tilde{Y}_{P}^{i, j+1}\right|$. Using the distances, we obtain the normalized weights: $w_{i, j-1}=\frac{\exp \left(-\Delta_{i, j-1}\right)}{\sum_{j \in 1, . .|G|}\left(\exp \left(-\Delta_{i, j-1}\right)+\exp \left(-\Delta_{i, j+1}\right)\right)}$, and $w_{i, j+1}=\frac{\exp \left(-\Delta_{i, j+1}\right)}{\sum_{j \in 1, . .|G|}\left(\exp \left(-\Delta_{i, j-1}\right)+\exp \left(-\Delta_{i, j+1}\right)\right)}$. For a given individual, say $P$, we consider the neighboring contexts by applying further weighting factors $\alpha_{1}, \alpha_{2}, \alpha_{3}$, (with $\alpha_{1}+\alpha_{2}+\alpha_{3}=1$ ), to control the impact of the three components of the neighboring contexts, where $\alpha_{1}$ is the weight on the estimated age $\tilde{Y}_{P}$ from second level estimation, $\alpha_{2}$ is the weight on the average predicted age for all individuals in the same probable age group as $P$, and $\alpha_{3}$ is the weight on the neighboring age groups of $P$. Then, we compute the final estimated age: $\hat{Y}=\alpha_{1} \tilde{Y}_{P}+\alpha_{2} \bar{G}_{P}+\alpha_{3} \bar{N}_{P}$, where $\bar{G}_{P}$ is the centroid of subject $P$ 's probable age group, and $\bar{N}_{P}$ captures the estimated ages $\left(\tilde{Y}_{P}^{i, j-1}\right.$, and $\tilde{Y}_{P}{ }^{i, j+1}$ ) from the left and right neighboring age groups, respectively, using Algorithm 1 . 
Here, $\bar{N}_{P}=\sum_{i=1}^{|C|}\left(w_{i, j-1} * \tilde{Y}_{P}^{i, j-1}+w_{i, j+1} * \tilde{Y}_{P}^{i, j+1}\right)$, where $j-1 \geq 1, j+1 \leq|G|$.

To find the best combination of $\alpha_{1}, \alpha_{2}, \alpha_{3}$ parameters that provides the lowest MAE on average, we selected these adaptively depending on the age group and body shape cluster of the subject under consideration. Thus, during training, we varied each of the $\alpha$ weights from 0 to 1 , and thus, for each given body shape cluster and age group, we compute the best set of $\left(\alpha_{1}, \alpha_{2}, \alpha_{3}\right)$ parameters that produced the best results. We record these in a look up table for later use. Algorithm 2 shows the pseudo code for the final stage of our multi-stage age estimation. Notice that the above formulation handles both cases with one neighbor, e.g., age groups $G_{1}$ or $G_{6}$, or those with two neighbors (e.g., age groups $G_{2}, G_{3}, G_{4}, G_{5}$ ).

For performance measurement, we use the notations, $Y=$ correct age, $\hat{Y}=$ final estimated age, and we compute the mean absolute error $\left(M A E=\frac{1}{n} \sum_{t=1}^{n}\left|Y_{t}-\hat{Y}_{t}\right|\right)$, root mean-square error $\left(R M S E=\sqrt{\frac{1}{n} \sum_{t=1}^{n}\left(Y_{t}-\hat{Y}_{t}\right)^{2}}\right)$, and correlation $\left(C O R R=\rho(Y, \hat{Y})=\frac{\operatorname{Cov}\left(Y_{t}, \hat{Y}_{t}\right)}{\sqrt{\operatorname{Var}\left(Y_{t}\right) \operatorname{Var}\left(\hat{Y}_{t}\right)}}\right)$ between the predicted age $\left(\hat{Y}_{t}\right)$ and the actual age $Y$, where $\operatorname{Cov}\left(Y_{t}, \hat{Y}_{t}\right)$ computes the covariance between $Y_{t}$ and $\hat{Y}_{t}$, and $\operatorname{Var}()$ computes the variance.

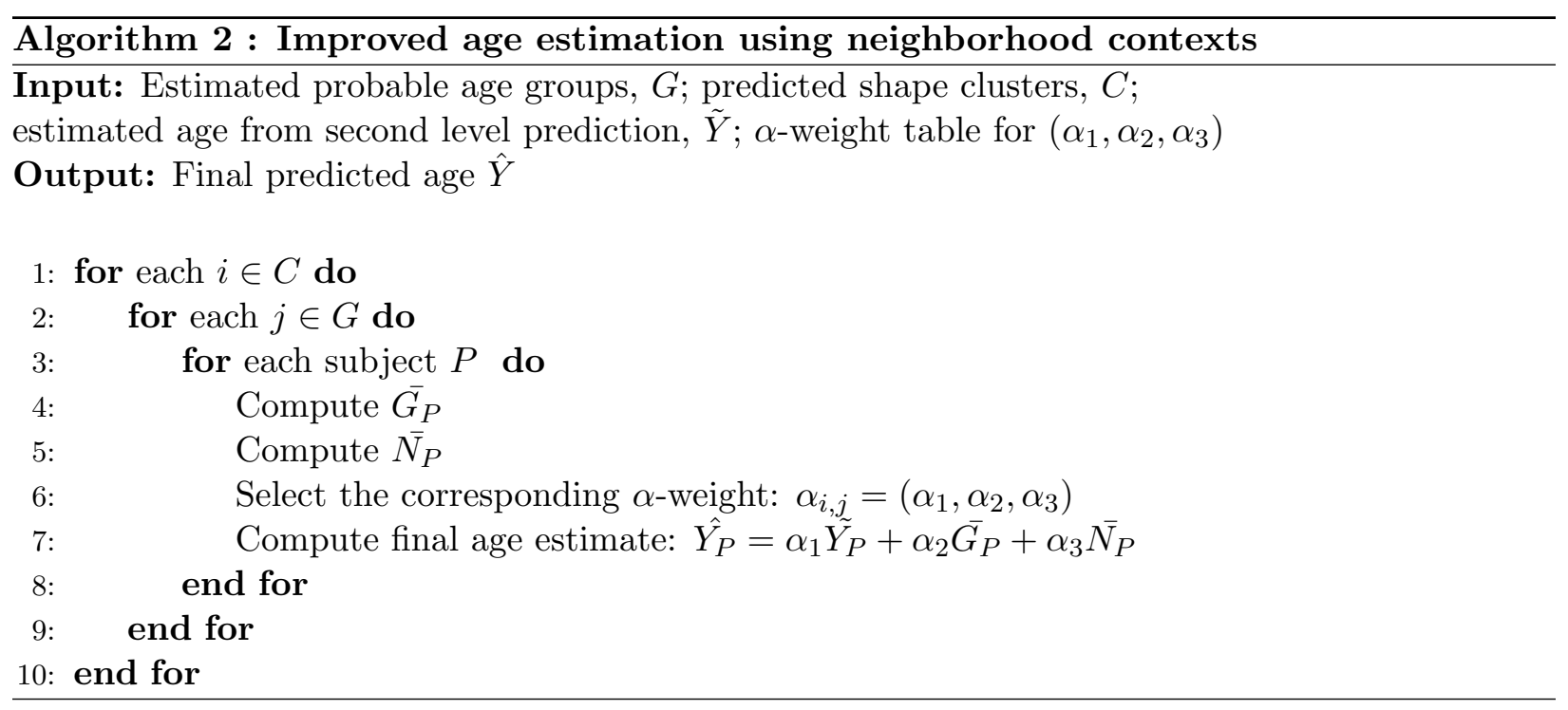




\subsection{Results}

We tested the performance of the proposed approach using the two datasets (NHANES, and CAESAR), as identified earlier. For the feature space, we used all the anthropometric measurements as indicated in Table 3.1. We applied four feature selection algorithms (forward, backward, and exhaustive, Lasso) 133,136 in an attempt to select the best feature subsets. We also applied a feature engineering approach by expanding the feature space using $z$-scores for certain measures, based on the results from the feature selection methods.

We tested the approach using $K$-fold cross valiation, where we set $K=10$. For $K$-fold cross-validation, the initial data is randomly partitioned into $K$ mutually exclusive subsets. Training and testing is performed $K$ times, whereby for each iteration $i$, the $i$ th partition is considered for testing, while the rest of the partitions are used for training. The reported results are then the averages from the $K$ iterations (folds). All statistical analyses were performed using the R Language, Ver. 3.3.4 (The R Foundation for Statistical Computing, Vienna, Austria). We tested our approach on three regression schemes, namely, SVM, RF, and LR. For SVM, we used the R package "e1071", with a radial basis function (RBF) kernel and a cost parameter $C=10$ ( $C$ was tuned using values: $0.001,0.01,0.1,1,5,10,100)$. For, $\gamma$, we have used default value which is $1 /$ (data dimension). For RF, we used the $\mathrm{R}$ package "randomForest". Based on empirical observations, we set the number of random attributes to be selected at each decision node to 13, and the number of trees to 100 . The initial results are based on the NHANES data set. We use the CAESAR dataset for cross-dataset evaluation of our method, and for comparative analysis.

\subsubsection{Baseline Results:}

Simple Prediction The original question was whether anthropometric measurements can predict age for adult humans. Thus the natural starting point is to apply simple linear prediction using the measurements. Table 3.3 shows the results for simple age prediction 
using the NHANES dataset. First, we use the original anthropometric measurements (for all 11808 people) in the dataset to predict age, using the 10-fold cross validation approach. We obtained a correlation coefficient of 0.72 and MAE of 11.31 using SVM for prediction. Then we apply PCA, selecting the first few principal components (PCs) with $97.96 \%$ variance explained. This resulted in 10 PCs for our analysis. MAE was reduced to 11.27 for SVM, and 11.57 using RF. See Table 3.3 .

We also attempted feature selection methods to improve the results. We considered four feature selection methods, namely, backward, forward, exhaustive, and Lasso feature selection 133,136]. Table S2 shows the feature rankings from each of the feature selection methods. Table S3 shows performance of the top 10 selected features for each of the four approaches. We observe that the results obtained from the four different feature selection algorithms are quite similar although the selected features are very different. For instance, using SVM we have (backward: MAE=11.56, $\rho=0.71$; forward: MAE=11.46, $\rho=0.71$; exhaustive: $\mathrm{MAE}=11.42, \rho=0.71$; and Lasso: $\mathrm{MAE}=12.00, \rho=0.67)$. The results are also not too different from the simple prediction results, using all the measurements. This again demonstrates the difficulty of the problem, showing that simply applying feature selection may not lead us to improved age estimation results.

Table 3.3: Simple Age Prediction. Results are shown for all test subjects, using three classification schemes.

\begin{tabular}{|l|l|l|l|l|l|l|l|l|l|}
\hline & \multicolumn{3}{|c|}{ Original Features } & \multicolumn{3}{c|}{ PCA } & \multicolumn{3}{c|}{ Original + PCA } \\
\hline ALL & CORR & MAE & RMSE & CORR & MAE & RMSE & CORR & MAE & RMSE \\
\hline LR & 0.67 & 12.37 & 15.33 & 0.67 & 12.48 & 15.42 & 0.71 & 11.71 & 14.65 \\
\hline SVM & 0.72 & 11.31 & 14.61 & 0.72 & 11.27 & 14.66 & 0.75 & 10.65 & 13.81 \\
\hline RF & 0.70 & 12.04 & 14.92 & 0.72 & 11.57 & 14.33 & 0.74 & 11.19 & 13.92 \\
\hline Average & 0.69 & 11.91 & 14.96 & 0.70 & 11.78 & 14.80 & 0.73 & 11.18 & 14.12 \\
\hline
\end{tabular}

Feature Engineering. To improve the prediction accuracy, we now consider feature engineering. New features are obtained by combining the original features with the principal components and the top 12 selected z-score features based on the average ranking of the first three feature selection methods. See Table S3. We then perform a similar experiment 
as described for baseline results. The new results are shown in Table 3.3 (last 3 columns). Feature engineering combining the original anthropometric features with PCA resulted in a slight improvement in performance, with MAE of 10.65 and $\rho=0.75$ using Support Vector Machine (SVM).

\subsubsection{Eigen Body Shape Clustering Improves Age Estimation Accuracy}

To test the performance of the proposed multi-level prediction, first we performed age grouping using an initial estimate of the age (using SVM regression). The initial age estimate is obtained from the first level age prediction, essentially using the feature engineering results with PCA. Based on the initial age estimate, we classified a given individual into one of the six age groups: $G_{1}(\leq 25), G_{2}(26-35), G_{3}(36-45), G_{4}(46-55), G_{5}(56-70), G_{6}(70+)$.

Number of body shape clusters. We applied body shape clustering based on the eigen body shape model and then performed estimation separately for each body shape cluster within a given age group, using RF. Fig. 3.3(a) shows the impact of the number of body shape clusters using the Davies-Bouldin (DB) index [41] and Dunn index [46], two different measures for evaluating the performance of clustering algorithms. These essentially consider how close items in the same cluster are (within-cluster distance), and how separated the items in different clusters are (between cluster distance). For a given clustering algorithm, a higher DB or Dunn index indicates better clustering performance. At $k=4$ we have DB index of 1.86 which is highest among $k=2$ to $k=6$. For Dunn Index, $k=2$ has the maximum value and $k=3$ to $k=5$ have similar values, around 1.16 . Given the above, we selected $k=4$ as our number of clusters (since $k=2$ generally corresponds to female and male grouping). Fig. 3.3(b) shows the estimation results (MAE) for all the age groups and each cluster. These accuracy results are computed based on the estimated age $\tilde{Y}_{P}$ from Algorithm 1 .

Age distribution for body shape clusters. Fig. 3.4 shows the distribution of age groups for the eigen bodyshape clusters, when PCA is used. Here, for each bodyshape cluster, the 




(a)

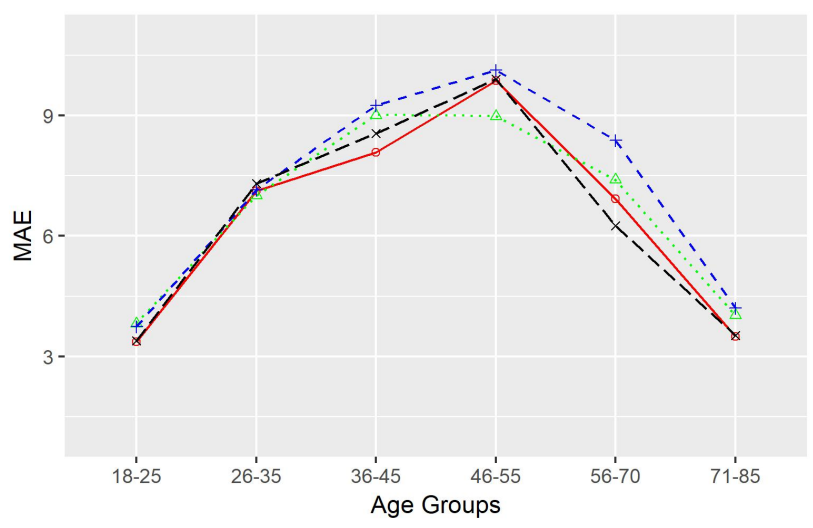

(c)



(b)
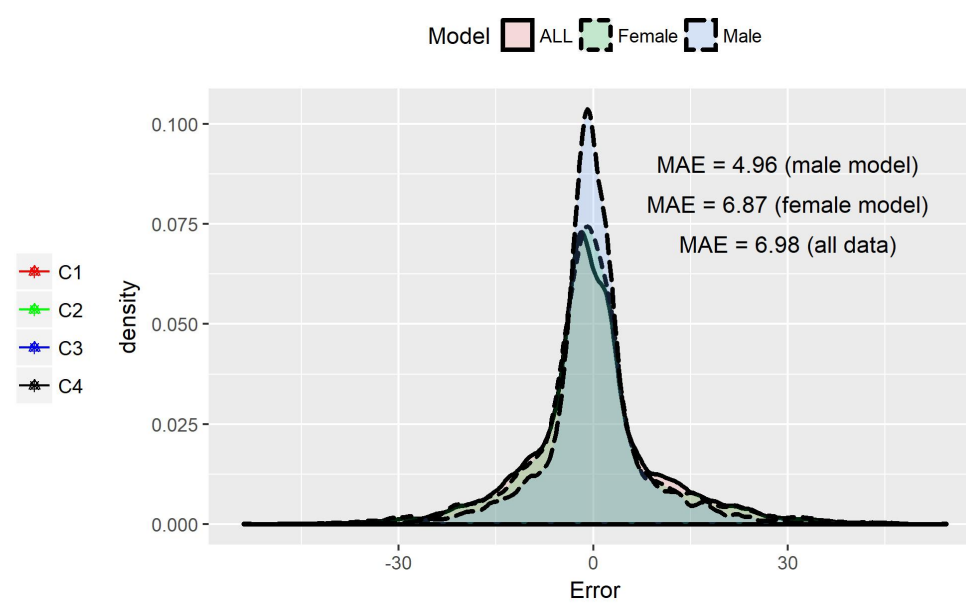

(d)

Figure 3.3: Impact of body shape clustering on age estimation performance: (a) effect of the number of shape clusters $k$; (b) performance variation with shape clusters $\left(C_{1}, C_{2}, C_{3}, C_{4}\right)$ and age groups $\left(G_{1}: \leq 25, G_{2}: 26-35, G_{3}: 36-45, G_{4}: 46-55, G_{5}: 56-70, G_{6}: 70+\right)$ without neighborhoodbased refinement; (c) performance variation with neighborhood-based refinement; (d) density of estimation error for the final estimated age using gender-specific models (male, female) and nongender specific model (labeled "all data"). Reported results are based on all test subjects using 10-fold cross validation. 
number of people in different age groups are plotted in the bar chart. Age group $G_{1}$ ( $\leq 25 y \mathrm{yr}$ ) had most number of people among the clusters. Groups $G_{2}, G_{3}, G_{4}, G_{5}$, and $G_{6}$ have about a similar number of people. From the figure we can see that these clusters have generally similar age distributions. We also observe that each shape cluster contains subjects at all age groups. This is expected since people of different ages could have similar body shapes. Thus, for improved age estimation, we build an estimation model for each age group within a given body shape cluster. Fig. 3.3 (b) shows the performance of the approach. The overall average results, across all age groups and shape clusters were $\mathrm{MAE}=6.96$; $\operatorname{Correlation}(\rho)=0.35$; $\mathrm{RMSE}=8.31$. Best results (minimum MAE) were obtained for age groups $G_{1}(\leq 25)$ and $G_{6}($ $>70$ ). Worst results (maximum MAE) were obtained for subjects in age groups $G_{3}(36-45)$ and $G_{4}(46-45)$. Given our earlier discussion under key observations and motivation, this is not surprising. For body shape clusters, the most challenging were clusters $C_{2}$ and $C_{3}$, while clusters $C_{1}$ and $C_{4}$ were relatively easier. These results are summarized in Table 3.4, under $\left(I D_{35}(1,0,0)\right)$.

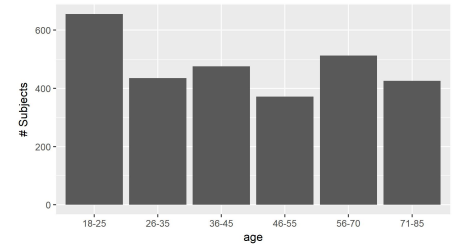

(a) Cluster 1

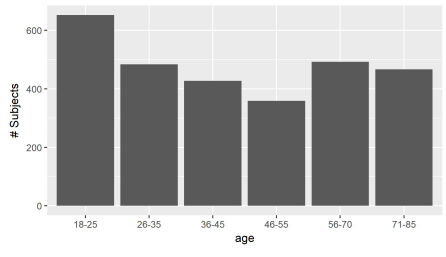

(b) Cluster2

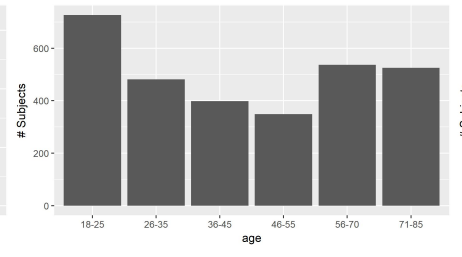

(c) Cluster3

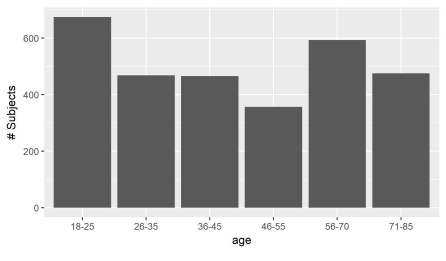

(d) Cluster4

Figure 3.4: Age group distribution for different body shape clusters.

\subsubsection{Neighborhood Contexts Further Improves Both Estimation Accuracy and Stability}

To test the performance of our proposed context-based refinement of the age estimates (Algorithm 2), we used different values of $\alpha_{1}(0,0.1,0.25,0.5,0.75,0.9,1)$, and $\alpha_{2}(0.5,0.45$, $0.375,0.25,0.125,0.05,0)$, and $\alpha_{3}$ was calculated from the relation $\left(\alpha_{3}=1-\left(\alpha_{1}+\alpha_{2}\right)\right)$. We have considered 35 possible $\alpha$ variations for these sets of values. We notice that with variation 
of $\alpha_{1}, \alpha_{2}$ and $\alpha_{3}$ using the neighborhood approach we are able to estimate age with better accuracy (lower MAE) than the initial predicted age from Algorithm 1. Fig. 3.3 (c) shows the variation of estimation errors (MAE) for different age groups and body shape clusters, using neighborhood-based refinement. Compared with Fig. 3.3(b), we observe that on average, after the neighborhood approach is applied, the prediction error (MAE) is reduced for each age group and each body shape cluster. Fig. 3.3(d) shows the density of the estimation errors using male and female and models separately, and for using non-gender specific model (labeled "all data").

Table 3.4 shows the detailed final estimation results from the proposed multi-stage approach, using 10-fold cross validation, for some of the 35 variations of $\alpha_{1}$ combinations, where $I D_{1}$ has value of $\left(\alpha_{1}, \alpha_{2}, \alpha_{3}\right)=(0,0.5,0.5), I D_{25}(0.5,0.25,0.25), I D_{35}(1,0,0)$. For the table, we notice that $I D_{35}\left(\alpha_{1}=1, \alpha_{2}=0, \alpha_{3}=0\right)$ corresponds to the case where neighborbood contexts were not considered (no refinement). Case $I D_{1}\left(\alpha_{1}=0, \alpha_{2}=0.5, \alpha_{3}=0.5\right)$ corresponds to the other extreme where the original 2nd level prediction $(\tilde{Y})$ is ignored, and final results are based on the neighboring contexts. Case with $I D_{25}\left(\alpha_{1}=0.5, \alpha_{2}=0.25, \alpha_{3}=0.25\right)$ corresponds to a middle ground approach involving both $\tilde{Y}$ and the neighboring contexts. In each of the above, the $\alpha$-values are applied to all neighbors, independent of their cluster or age group. In our proposed adaptive approach (last column), we empirically choose the $\alpha$-parameters adaptively, based on the body shape clusters and age groups. Table 3.5 shows the corresponding $\alpha$-parameters used in the adaptive approach.

Another key issue in age estimation is stability of the results. Table 3.4 includes the standard deviation (SD) along with the MAE for each age group and each cluster. We observe that, on average using Random Forest at second-level age estimation (without neighborhood refinement), i.e., $\left.I D_{35}(1,0,0)\right)$ resulted in an average group MAE of $\left.6.96 \pm 6.69\right)$. Applying the proposed neighborhood approach with adaptive weights led to a lower average group MAE of $6.70 \pm 5.87$ (last column in Table 3.4). Thus the proposed approach achieves more stable results (lower standard deviation) and also more accurate estimation (lower MAE). 
We observe that, although for some cases the second-level estimate $\left(I D_{35}(1,0,0)\right)$ has lower MAE, for most cases different weight combinations result in improved results, over second-level prediction performance.

Fig. S3 (in Supplementary Material) shows the distribution of estimation errors for each age group and body shape cluster using gender-specific models (separate models for male and female, respectively). Age groups $G_{1}, G_{5}$, and $G_{6}$ showed generally similar error distributions for male and female subjects. Error distribution is also generally similar among the shape clusters. However, we observe that the distribution tended to vary between male and female subjects for age groups $G_{2}, G_{3}$, and $G_{4}$. This is expected as these three groups have higher group MAEs for female in comparison with similar male groups. The shape clusters have different distribution for these groups.

\subsubsection{Impact of Gender.}

As observed in the introduction, gender is expected to have some influence on the performance of an age estimation scheme. Thus, most studies have applied different models for female and male. As discussed earlier, simple age prediction resulted in an MAE of 13.09 for female subjects, and 9.67 for males, using SVM. Also at second-level estimation, we observe that female age estimation is more difficult than the corresponding male group. Table 3.6 shows the results of our multi-stage approach, for the cases of using different models for male and female for each age group and body shape model (labelled "Male" and "Female", respectively). Among all the age groups, $G_{6}$ (>70yrs) had the lowest variation of results (estimation error) between female and male groups, followed by $G_{1}$. Expectedly, $G_{3}$ and $G_{4}$ had the largest variation. On average, we obtain average MAE of 6.40 for female (average of group average MAEs), and average MAE of 4.86 for male, using our proposed adaptive neighborhood contexts (second to last row). The last row in Table 3.6 shows the overall

performance of our proposed approach, using 10-fold cross validation. Here, the MAEs are based on the overall data, rather than average of group averages. We obtain an overall 
Table 3.4: Improving estimation accuracy and stability using neighborhood contexts. Results (mean absolute error (MAE)) are shown for all subjects, using 10-fold cross validation, where $I D_{1}$ corresponds to using $\left(\alpha_{1}, \alpha_{2}, \alpha_{3}\right)=(0,0.5,0.5), I D_{25}(0.5,0.25,0.25), I D_{35}(1,0,0)$. Results are grouped by the probable age groups (based on first level of age estimation).

\begin{tabular}{|c|c|c|c|c|}
\hline ALPHA & $I D_{1}(0,0.5,0.5)$ & $I D_{25}(0.5,0.25,0.25)$ & $I D_{35}(1,0,0)$ & Adaptive (Proposed Approach) \\
\hline \multicolumn{5}{|l|}{$\leq 25$} \\
\hline$C_{1}$ & $5.53 \pm 3.78$ & $4.28 \pm 4.07$ & $3.37 \pm 4.57$ & $3.37 \pm 3.75$ \\
\hline$C_{2}$ & $5.53 \pm 3.64$ & $4.58 \pm 4.06$ & $3.86 \pm 4.57$ & $3.82 \pm 3.39$ \\
\hline$C_{3}$ & $5.63 \pm 3.82$ & $4.59 \pm 4.28$ & $3.82 \pm 4.93$ & $3.74 \pm 3.70$ \\
\hline$C_{4}$ & $5.59 \pm 3.54$ & $4.39 \pm 3.80$ & $3.39 \pm 4.43$ & $3.39 \pm 3.52$ \\
\hline \multicolumn{5}{|l|}{$26-35$} \\
\hline$C_{1}$ & $7.28 \pm 6.27$ & $7.17 \pm 6.42$ & $7.26 \pm 6.63$ & $7.13 \pm 6.27$ \\
\hline$C_{2}$ & $7.03 \pm 6.48$ & $7.06 \pm 6.56$ & $7.25 \pm 6.79$ & $7.01 \pm 6.47$ \\
\hline$C_{3}$ & $7.18 \pm 6.37$ & $7.20 \pm 6.47$ & $7.35 \pm 6.65$ & $7.15 \pm 6.37$ \\
\hline$C_{4}$ & $7.34 \pm 6.48$ & $7.32 \pm 6.65$ & $7.45 \pm 6.91$ & $7.30 \pm 6.48$ \\
\hline \multicolumn{5}{|l|}{$36-45$} \\
\hline$C_{1}$ & $8.10 \pm 7.92$ & $8.23 \pm 8.03$ & $8.63 \pm 8.14$ & $8.08 \pm 7.91$ \\
\hline$C_{2}$ & $9.04 \pm 8.20$ & $9.10 \pm 8.07$ & $9.36 \pm 8.07$ & $9.01 \pm 8.04$ \\
\hline$C_{3}$ & $9.39 \pm 8.47$ & $9.33 \pm 8.36$ & $9.59 \pm 8.38$ & $9.24 \pm 8.34$ \\
\hline$C_{4}$ & $8.75 \pm 8.26$ & $8.64 \pm 8.18$ & $8.90 \pm 8.15$ & $8.55 \pm 8.14$ \\
\hline \multicolumn{5}{|l|}{$46-55$} \\
\hline$C_{1}$ & $9.97 \pm 9.12$ & $9.94 \pm 9.07$ & $10.24 \pm 9.10$ & $9.86 \pm 9.06$ \\
\hline$C_{2}$ & $9.10 \pm 8.46$ & $9.13 \pm 8.47$ & $9.54 \pm 8.51$ & $8.98 \pm 8.44$ \\
\hline$C_{3}$ & $10.28 \pm 9.39$ & $10.21 \pm 9.29$ & $10.55 \pm 9.21$ & $10.12 \pm 9.21$ \\
\hline$C_{4}$ & $9.95 \pm 8.57$ & $10.08 \pm 8.73$ & $10.44 \pm 8.93$ & $9.91 \pm 8.56$ \\
\hline \multicolumn{5}{|l|}{$56-70$} \\
\hline$C_{1}$ & $7.31 \pm 7.47$ & $7.05 \pm 7.41$ & $7.25 \pm 7.33$ & $6.92 \pm 7.32$ \\
\hline$C_{2}$ & $7.65 \pm 7.37$ & $7.58 \pm 7.40$ & $7.82 \pm 7.48$ & $7.40 \pm 7.36$ \\
\hline$C_{3}$ & $8.73 \pm 8.41$ & $8.51 \pm 8.40$ & $8.78 \pm 8.53$ & $8.38 \pm 8.25$ \\
\hline$C_{4}$ & $6.73 \pm 6.80$ & $6.41 \pm 6.80$ & $6.44 \pm 6.89$ & $6.25 \pm 6.78$ \\
\hline \multicolumn{5}{|l|}{$70+$} \\
\hline$C_{1}$ & $6.28 \pm 3.72$ & $4.61 \pm 3.20$ & $3.56 \pm 3.40$ & $3.50 \pm 3.18$ \\
\hline$C_{2}$ & $6.64 \pm 4.45$ & $5.19 \pm 4.33$ & $4.14 \pm 4.70$ & $4.02 \pm 4.29$ \\
\hline$C_{3}$ & $6.61 \pm 4.32$ & $5.21 \pm 4.30$ & $4.43 \pm 4.54$ & $4.21 \pm 4.24$ \\
\hline$C_{4}$ & $5.81 \pm 3.98$ & $4.53 \pm 3.64$ & $3.71 \pm 3.83$ & $3.52 \pm 3.64$ \\
\hline Group average & $7.56 \pm 6.47$ & $7.10 \pm 6.02$ & $6.96 \pm \mathbf{6 . 6 9}$ & $\mathbf{6 . 7 0} \pm \mathbf{5 . 8 7}$ \\
\hline Non Group average & $7.67 \pm 7.10$ & $7.31 \pm 7.22$ & $7.25 \pm 7.48$ & $6.98 \pm 7.33$ \\
\hline
\end{tabular}

Table 3.5: Weights for adaptive neighborhood-based refinement.

\begin{tabular}{|l|l|l|l|l|l|l|}
\hline$\alpha_{1}, \alpha_{2}, \alpha_{3}$ & $G_{1}(\leq \mathbf{2 5})$ & $G_{2} \mathbf{( 2 6 - 3 5 )}$ & $G_{3} \mathbf{( 3 6 - 4 5 )}$ & $G_{4}(\mathbf{4 6 - 5 5 )}$ & $G_{5}(\mathbf{5 6 - 7 0})$ & $G_{6}(\mathbf{7 1 - 8 5 )}$ \\
\hline$C_{1}$ & $1,0,0$ & $0.5,0.5,0$ & $0.1,0.5,0.4$ & $0.25,0.5,0.25$ & $0.5,0.5,0$ & $0.75,0.25,0$ \\
\hline$C_{2}$ & $0.5,0.5,0$ & $0.25,0.5,0.25$ & $0.25,0.5,0.25$ & $0.25,0.5,0.25$ & $0.5,0.5,0$ & $0.5,0.5,0$ \\
\hline$C_{3}$ & $0.5,0.5,0$ & $0.25,0.5,0.25$ & $0.25,0.5,0.25$ & $0.25,0.5,0.25$ & $0.5,0.5,0$ & $0.5,0.5,0$ \\
\hline$C_{4}$ & $1,0,0$ & $0.25,0.45,0.30$ & $0.5,0.5,0$ & $0.25,0.5,0.25$ & $0.5,0.5,0$ & $0.5,0.5,0$ \\
\hline
\end{tabular}


MAE of 6.87 for females, and 4.96 for males, or an overall MAE of 5.90 over the entire dataset (with 5840 females, 5968 males). The results are consistent with other existing work that show that estimating age for female subjects is generally more difficult than for male subjects $54,62,65]$.

\subsection{Discussion}

\subsubsection{Cross Database Evaluation}

To test the generality of the method we propose, we applied our approach to measure the performance on the CAESAR dataset. As shown in Supplementary Material (Fig. S2), the age range for the CAESAR dataset differs from those from NHANES. Thus for our analysis we removed the two boundary age groups $G_{1}$ and $G_{6}$ (Although in $G_{1}$ CAESAR have some people, those are only in cluster3 and cluster4, we did not apply our proposed method for the sake of generality). Hence, for our neighborhood-based age prediction model, the boundary groups will be $G_{2}$ and $G_{5}$ while $G_{3}, G_{4}$ works as before. We have employed two different approaches for performance measurement on CAESAR dataset. First, we trained the age group and body shape cluster models using the NHANES dataset, and then tested the models using the CAESAR dataset. Second, we applied proposed approach directly on the CAESAR dataset, using 10-fold cross validation. Using the first approach resulted in an average group MAE of 4.38 using RF for regression. The second approach resulted in an average group MAE of 2.71 which is reduced to 2.58 after neighborhood-base refinement. The MAEs over the entire dataset (without group averages) were 4.62 for the first method, and 2.33 for the second method. These results show the generality of the approach, and the potential for improved results with intelligent stratification. In this work, we did not consider potential impact of other factors such as race or ethnicity. The data sets we used are predominantly white, but we believe the approach can easily be applied independent of race/ethnicity with enough training data. 
Table 3.6: Summary results showing the impact of gender on the performance of the proposed neighborhood-based refinement for age estimation. Results (mean absolute error (MAE)) are shown for female-only and male-only subjects, using 10-fold cross validation, where $I D_{35}$ corresponds to using $\left(\alpha_{1}, \alpha_{2}, \alpha_{3}\right)=(1,0,0)$.

\begin{tabular}{|c|c|c|c|c|}
\hline ALPHA & \multicolumn{2}{|c|}{ Female } & \multicolumn{2}{|c|}{ Male } \\
\hline$\leq 25$ & $I D_{35}(1,0,0)$ & Adaptive & $I D_{35}(1,0,0)$ & Adaptive \\
\hline$C_{1}$ & $4.18 \pm 5.16$ & $4.18 \pm 3.86$ & $2.99 \pm 2.93$ & $2.99 \pm 3.12$ \\
\hline$C_{2}$ & $2.93 \pm 4.25$ & $2.93 \pm 3.10$ & $2.09 \pm 1.98$ & $2.09 \pm 2.24$ \\
\hline$C_{3}$ & $1.41 \pm 1.53$ & $1.41 \pm 1.38$ & $1.88 \pm 1.95$ & $1.88 \pm 2.26$ \\
\hline$C_{4}$ & $3.65 \pm 5.27$ & $3.65 \pm 4.08$ & $2.17 \pm 2.40$ & $2.17 \pm 2.60$ \\
\hline \multicolumn{5}{|l|}{$26-35$} \\
\hline$C_{1}$ & $7.12 \pm 7.14$ & $6.83 \pm 6.63$ & $6.80 \pm 6.47$ & $6.28 \pm 6.55$ \\
\hline$C_{2}$ & $7.53 \pm 7.52$ & $7.34 \pm 6.80$ & $4.04 \pm 3.40$ & $3.94 \pm 3.72$ \\
\hline$C_{3}$ & $5.36 \pm 6.60$ & $5.24 \pm 6.08$ & $5.63 \pm 4.95$ & $5.52 \pm 5.12$ \\
\hline$C_{4}$ & $7.60 \pm 7.23$ & $7.40 \pm 6.69$ & $5.60 \pm 5.67$ & $5.52 \pm 5.83$ \\
\hline \multicolumn{5}{|l|}{$36-45$} \\
\hline$C_{1}$ & $8.85 \pm 8.08$ & $8.48 \pm 7.91$ & $6.64 \pm 6.48$ & $6.09 \pm 6.56$ \\
\hline$C_{2}$ & $11.19 \pm 9.93$ & $9.84 \pm 9.36$ & $5.90 \pm 6.64$ & $5.75 \pm 6.84$ \\
\hline$C_{3}$ & $7.92 \pm 8.32$ & $7.66 \pm 8.00$ & $5.77 \pm 5.87$ & $5.56 \pm 5.92$ \\
\hline$C_{4}$ & $10.33 \pm 9.27$ & $9.56 \pm 8.96$ & $7.52 \pm 7.43$ & $7.00 \pm 7.49$ \\
\hline \multicolumn{5}{|l|}{$46-55$} \\
\hline$C_{1}$ & $10.33 \pm 9.23$ & $9.85 \pm 9.23$ & $7.31 \pm 6.76$ & $6.96 \pm 7.24$ \\
\hline$C_{2}$ & $10.30 \pm 9.61$ & $9.97 \pm 9.48$ & $7.53 \pm 7.74$ & $7.22 \pm 7.82$ \\
\hline$C_{3}$ & $9.62 \pm 8.67$ & $9.08 \pm 8.32$ & $6.53 \pm 5.92$ & $6.42 \pm 5.96$ \\
\hline$C_{4}$ & $11.18 \pm 9.06$ & $10.67 \pm 8.84$ & $8.33 \pm 7.34$ & $6.92 \pm 7.55$ \\
\hline \multicolumn{5}{|l|}{$56-70$} \\
\hline$C_{1}$ & $6.83 \pm 7.69$ & $6.49 \pm 7.62$ & $6.81 \pm 7.05$ & $6.35 \pm 7.16$ \\
\hline$C_{2}$ & $7.06 \pm 7.90$ & $6.88 \pm 7.60$ & $4.46 \pm 5.16$ & $4.18 \pm 5.33$ \\
\hline$C_{3}$ & $5.40 \pm 5.62$ & $5.06 \pm 5.59$ & $6.01 \pm 6.28$ & $5.67 \pm 6.58$ \\
\hline$C_{4}$ & $7.61 \pm 8.52$ & $7.32 \pm 8.52$ & $5.85 \pm 5.74$ & $5.51 \pm 6.00$ \\
\hline \multicolumn{5}{|l|}{$70+$} \\
\hline$C_{1}$ & $4.08 \pm 5.87$ & $3.98 \pm 5.63$ & $3.53 \pm 3.92$ & $3.44 \pm 4.12$ \\
\hline$C_{2}$ & $3.03 \pm 3.05$ & $3.03 \pm 2.79$ & $3.28 \pm 3.19$ & $3.17 \pm 3.58$ \\
\hline$C_{3}$ & $3.72 \pm 2.96$ & $3.31 \pm 2.65$ & $3.00 \pm 2.54$ & $3.00 \pm 3.22$ \\
\hline$C_{4}$ & $3.41 \pm 3.56$ & $3.41 \pm 3.26$ & $3.09 \pm 2.93$ & $3.09 \pm 3.54$ \\
\hline Group average & $6.69 \pm 6.75$ & $6.40 \pm 6.35$ & $5.12 \pm 5.03$ & $4.86 \pm 5.26$ \\
\hline Non Group average & $7.18 \pm 7.89$ & $6.87 \pm 7.71$ & $5.20 \pm 5.85$ & $4.96 \pm 5.78$ \\
\hline
\end{tabular}




\subsubsection{Comparative Results}

Our proposed method has a comparable accuracy with other methods that have used different approaches. Table 3.7 shows the comparative performance of different popular methods along with the various features used, dataset used, and the year the method was introduced. The results show that our proposed approach using 2-level age estimation with neighborhood refinement (with an overall MAE of 5.90) is very competitive when compared with other previous methods. Also we have included baseline results using direct measurements (MAE $=11.31$ with SVM), and using PCA on the measurements (MAE = 11.27, using SVM).

Table 3.7: Comparative results of Age Prediction.

\begin{tabular}{|c|c|c|c|c|c|}
\hline Publication/Work & Features & Dataset & \# Subjects & Year & Accuracy(MAE) \\
\hline Guo et al $\overline{59}$ & Face (Regression based) & YGA & 8000 & 2008 & $6(\mathrm{M}), 5.5(\mathrm{~F})$ \\
\hline $\mathrm{Ni}$ et al $1 \overline{2}{ }^{3}$ & Face(Appearance Model) & Web, FG-NET & 78711 & 2009 & 7.42 \\
\hline Lu et al $\overline{92}$ & Gait Sequence & GEI & 1870 & 2010 & 3.02 \\
\hline Shamir et al 128 & Hand Photo & BLSA & 212 & 2011 & $88 \%$ \\
\hline Guo and Wang 60 & Face(Kernel PlS) & MORPHII & 55000 & 2012 & 4.2 \\
\hline Geng et al 56 & Face(Appearance \& Bio Inspired) & MORPHII & 55132 & 2013 & 4.8 \\
\hline Horvath $70^{\circ}$ & Epigenetic(DNA methylation) & Illumina & 82 & 2013 & $4.2(\mathrm{MnAE})$ \\
\hline Wang et al 139 & Face (Deep Learned Aging Pattern) & FGNET, MORPH & 515,82 & 2015 & FG-4.26, MO-4.77 \\
\hline Putin et al 114 & Biomarkers(Deep Learning) & Invitro Lab, Ltd & 62419 & 2016 & 5.55 \\
\hline Eipel et al 48 & Epigenetic(DNA methylation) & Own Collection & 55 & 2016 & 7.03 \\
\hline Cole et al 38 & Brain Image & LBC1936 & 1091 & 2017 & 7.08 \\
\hline BaseLine (direct features) & Anthropometric & NHANES & $11808(5840 \mathrm{f}, 5968 \mathrm{~m})$ & & 11.31 \\
\hline BaseLine (PCA) & Anthropometric & NHANES & $11808(5840 f, 5968 \mathrm{~m})$ & & 11.27 \\
\hline Proposed (10-fold cross validation) & Anthropometric & NHANES & 11808 & & 5.90 \\
\hline Proposed(10-fold cross validation) & Anthropometric & CAESAR & 2390 & & 2.33 \\
\hline Proposed (Train, Test ${ }^{*}$ ) & Anthropometric & CAESAR & 2390 & & 4.62 \\
\hline
\end{tabular}

* Train on NHANES, test on CAESAR (see text), FG, MO denote FGNET and MORPH data, respectively; MnAE $=$ Median Absolute Error; Accuracy is measured in percentile in Shamir et al 128.

The comparative results show that the performance of our proposed multi-stage approach to age estimation using anthropometric features is quite competitive. At an MAE of 5.90, the results are comparable with those of recent face/image-based methods (MAE 4.2 to 7.42), and those with biomarkers (MAE $=5.55$ ), or epigenetics (MAE 7.03 on a small dataset). We acknowledge the limitations of comparing several methods based on results on different datasets with potentially different structure and statistics. Yet, even only considering the average performance of all these methods (MAE $=5.23)$ against our results shows the merit of the proposed multi-stage approach to age estimation.

From the foregoing, the original question of whether human anthropometric measurements 
can reliably predict adult age can be answered in the affirmative. Further, since anthropometric features tend to perform best in the age ranges that are most challenging for image/face-based methods (e.g., children and adolescents, and the elderly above 70), a combination of these two approaches may be beneficial when the required measurements are available.

\subsubsection{Impact of Age Grouping and Feature Interaction}

As described earlier, we have tried different feature selection methods, including backward feature selection, forward feature selection, and exhaustive feature selection algorithms. We also tried Lasso regression, which performs variable selection, much like subset selection. Table S2 shows the feature ranking by each of these feature selection algorithms, while Table S3 shows the age estimation results when we use the top 10 features according the the given ranking. We observe that these algorithms generally have similar performance for age prediction, although the top ranked features could vary significantly. The reason is the significant correlation and higher-order interactions between these measurements, which are difficult to distangle using direct feature selection approaches.

Also, as expected, the initial age grouping can have some impact on the results. For instance, we have tried two different age grouping. The results shown thus far is based on the groups: $G_{1}(\leq 25), G_{2}(26-35), G_{3}(36-45), G_{4}(36-45), G_{5}(56-70), G_{6}(71-85)$. We have also applied the age grouping described by Rahman and Adjeroh [118], which was given as follows: $G_{1}(\leq 20), G_{2}(21-35), G_{3}(36-50), G_{4}(51-70), G_{5}(71-85)$. Using this age grouping, with some change in the $z$-score fetures used (here, using $z$-scores for weight, height, BMI, WC, WHR, VTC, ABSI, SBSI), we obained an average group MAE of 5.40 without a genderspecific model. Using gender-specific models for male and female subjects, we obtained a group average MAE of 4.48, and an overall MAE of 5.68 (without group averages). This is slightly better than the results with the proposed age grouping. A potential future work could be to study in more detail, how to tune different parameters in the algorithm for 
improved performance. 


\section{Chapter 4}

\section{Centroid of Age Neighborhoods for Biological Age Estimation}

\subsection{Introduction}

Human age estimation is an important problem that has witnessed an increased attention, given its role in various daily activities, from health assessment [94], to social interaction [103], to security and identity profiling [57]. Although age estimation has been practiced for centuries, accurate age estimation is known to be a difficult problem. Doing this automatically by a machine is an even more onerous task [57,65]. The major challenge is that most of the various measures used to characterize age, for instance, visual appearance, and biological/physiological markers vary significantly from person to person, even for people of the same chronological age. The reason is that there are many unknowns (e.g., genetics [48, 145], nutrition [125], body shape [118], health condition [125], social conditions [71], life style [36], weather [13], and even cultural considerations [13]), contribute to influence the perceived age of an individual.

Age has a deep connection with health and mortality 21,89, 97]. Aging is a gradual process that results in increased health risk, and mortality over time. In general, a younger person is expected to have a better health condition and his/her mortality hazard should be low in comparison with a relatively older person. But two different people of the same age may 
have very different health conditions and mortality hazards. This brings up the debate on "chronological" versus "biological" age.

Chronological age is typically what we know and is based on the date of birth. However, biological age is based on the interesting, yet confounded, idea that a person's true age can be different from his/her chronological age. Biological age (sometimes called functional age [129]) lacks a precise definition, but it is often viewed as the true age of an individual in the gerontology and aging research community [83]. The common idea is that, biological age provides a better estimator of the true life expectancy of the individual than his or her chronological age. Quantification of biological age is a difficult challenge, since there is no well defined criteria. To estimate biological age, some age-dependent variables are used [35, 74, 127], and chronological age may or may not be a required attribute/variable depending on the application.

Klemera and Doubal's approach [83] is the most popular, and perhaps, the most effective biological age estimation method $21,89,97]$. The biological age (BA) estimates are derived based on minimizing the distance between biomarker points and regression lines, and the estimation is performed in a dimensional space of blood biomarkers. Other approaches include multiple linear regression (MLR) [89], principal component analysis (PCA) 87], and combination of MLR with PCA features [89]. Levine [89] compared the performance of five BA algorithms in terms of their ability to predict mortality. Klemera and Doubal's (KD) method was found to be the most reliable predictor for mortality. Overall, the performance of biological age (BA) in mortality prediction was significantly better than using chronological age $(\mathrm{CA})$. Cho et al 35 studied various BA estimation methods to examine the relation with work ability index (WAI). WAI is a measure that reflects present health condition rather than how it changes with age and their analysis showed that the KD method on PCA features produced the most reliable results. Mitnitski et al [97] compared the performance of the frailty index (FI) with biomarker-based measures of BA. They employed the KD algorithm in predicting mortality. Belsky et al [21] described biological age as a reflection 
of ongoing longitudinal change within a person. They estimated the BA for subjects at age 38 using the Klemera-Doubal equation with parameters estimated from the NHANESIII dataset. The study also tested the hypothesis that young adults with older biological age at age 38 years were actually aging faster than those with a younger biological age. They analyzed within-individual longitudinal change in 18 biomarkers from the Dunedin Study 113 across chronological ages 26 y, 32 y, 38 y to quantify each study member's personal rate of physiological deterioration. Cole et al [38] studied the use of structural neuro-imaging such as MRI under a Gaussian process regression framework to estimate biological age. The predicted age was identified as "brain-predicted age" or "brain age" for short. They combined DNA-methylation with brain age and showed that the combination improved mortality risk prediction. However, combining brain age with grey matter and cerebrospinal fluid volumes did not improve mortality risk prediction.

Other methods that have been used to assess biological age or speed of aging includes handgrip strength [124], locomotor activity [115], and deep learning on biomarker data [114. There is no clear consensus on which method is best for BA estimation, nor on how best to quantify the BA itself. Although KD method is the most popular, it is limited to biomarker features. Thus, biological age estimation in humans remains a significant challenge and evaluating the estimated $\mathrm{BA}$ is still a difficult problem. In this work, we introduce a novel centroid based method to estimate biological age.

Mitnitski et al 97 defined biological age acceleration as $\Delta=C A-B A$, where CA denotes chronological age and BA denotes biological age. However, we introduce the normalized form which we call normalized biological age acceleration (NBAA), denoted $\eta=\frac{\Delta}{C A}=\frac{C A-B A}{C A}$. This normalization is performed to reduce the effect of large $\Delta \mathrm{s}$. For example, a $\Delta$ value of 5 could have different biological or health implications for an 18 year old $(\approx 28 \%$ difference) and for a 70 year old ( $\approx 7 \%$ difference). Our observation is that, overall, using $\eta$ values provided more improvements in the results when compared to using $\Delta$ values. 
Another important observation is that although we estimate biological age based on minimizing the error between chronological age, the difference between the estimated age and the chronological age is our focus when considering the biological age.

\subsection{Methodology}

\subsubsection{Dataset}

We used biomarkers from the National Health and Human Nutrition Examination Surveys (NHANES) 1999-2010 [9]. NHANES employs a complex cluster design to sample members of the civilian USA population who are not institutionalized. NHANES uses stratified multistage probability to sample the data. Ethnicity included white, black, Mexican and others. For biomarkers of aging, we considered 16 of the biomarkers available in NHANES namely, C-reactive protein, glycated hemoglobin, albumin, total cholesterol, urea nitrogen, alkaline phosphatase, systolic blood pressure, diastolic blood pressure, pulse, high density lipoprotein, hemoglobin, lymphocyte percent, while blood cell count, hematocrit, red blood cell count, platelet count. Subsets of these have been used in earlier work as key biomarkers of biological age $21,89,127$. To begin with, we had 62160 individuals from year 1999 to 2010 dataset. We merged the datasets of different years and then performed matching with the mortality follow-up data that was updated in 2015. Thus, we obtained 21451 individuals with 1664 deaths during the 4-16 years of follow-up (1999-2015). Table 5.1 shows some information on the key biomarkers used in this study.

\subsubsection{Characteristics of the Dataset or Sample}

Table 4.2 shows the correlation between age and biomarkers. The table shows the correlation using direct measurements for both Pearson's $\rho$ (upper half), and Kendall's $\tau$ (lower half).

Table 5.1 also shows the correlation between chronological age and biomarkers. The table shows the correlation using direct measurements for both Pearson's $\rho$, and Kendall's $\tau$. Age 
Table 4.1: Key biomarker attributes for study participants of NHANES dataset.

\begin{tabular}{|l|l|c|c|}
\hline & & \multicolumn{2}{|c|}{ Correlation with Age } \\
\hline Biomarkers (N=21451) & Average \pm SD & $\rho$ & $\tau$ \\
\hline C-reactive protein & $0.38 \pm 0.78$ & 0.09 & 0.13 \\
\hline Glycated hemoglobin & $5.50 \pm 0.91$ & 0.33 & 0.35 \\
\hline Serum Albumin & $4.26 \pm 0.37$ & -0.17 & -0.15 \\
\hline Total Cholesterol & $197.77 \pm 42.27$ & 0.22 & 0.19 \\
\hline Serum Urea Nitrogen & $13.32 \pm 5.55$ & 0.44 & 0.31 \\
\hline Serum Alkaline Phosphatase & $74.75 \pm 28.00$ & 0.06 & 0.06 \\
\hline Systolic blood pressure & $123.88 \pm 20.02$ & 0.54 & 0.38 \\
\hline Diastolic blood pressure & $70.09 \pm 13.05$ & 0.07 & 0.11 \\
\hline Pulse & $72.12 \pm 12.21$ & -0.14 & -0.08 \\
\hline High density lipoprotein & $53.36 \pm 15.91$ & 0.05 & 0.02 \\
\hline Hemoglobin & $14.26 \pm 1.53$ & -0.07 & -0.04 \\
\hline Lymphocyte percent & $30.60 \pm 8.71$ & -0.10 & -0.07 \\
\hline White blood cell count & $7.09 \pm 2.42$ & -0.06 & -0.04 \\
\hline Hematocrit & $42.00 \pm 4.39$ & -0.06 & -0.03 \\
\hline Red blood cell count & $4.68 \pm 0.51$ & -0.17 & -0.1 \\
\hline Platelet count & $261.62 \pm 67.85$ & -0.12 & -0.08 \\
\hline Age(years) & $46.37 \pm 19.74$ & & \\
\hline
\end{tabular}

Table 4.2: Correlation coefficient (Pearson's $\rho$ ) between biomarkers and between biomarkers and age. Pearson $\rho$ (upper half), and Kendall $\tau$ (lower half).

\begin{tabular}{|c|c|c|c|c|c|c|c|c|c|c|c|c|c|c|c|c|c|}
\hline & CRP & GH & SAL & TC & SBU & SAP & SY1 & DI & PLS & HDL & HGB & LYP & WBC & HCT & RBC & $\overline{\text { PLT }}$ & AGE \\
\hline CRP & 1 & 0.09 & -0.25 & 0.02 & 0.01 & 0.15 & 0.05 & -0.04 & 0.15 & -0.04 & -0.12 & -0.16 & 0.18 & -0.11 & -0.08 & 0.10 & 0.09 \\
\hline GH & 0.12 & 1 & -0.11 & 0.10 & 0.18 & 0.13 & 0.22 & 0.03 & 0.03 & -0.12 & -0.01 & 0.03 & 0.04 & 0.00 & 0.04 & 0.00 & 0.33 \\
\hline SAL & -0.27 & -0.11 & 1 & -0.05 & 0.05 & -0.08 & -0.02 & 0.12 & -0.12 & -0.09 & 0.43 & 0.11 & -0.09 & 0.43 & 0.39 & -0.04 & -0.17 \\
\hline TC & 0.11 & 0.11 & -0.03 & 1 & 0.02 & 0.07 & 0.17 & 0.16 & 0.07 & 0.18 & 0.06 & 0.00 & 0.04 & 0.05 & 0.02 & 0.10 & 0.22 \\
\hline SBU & 0.02 & 0.17 & 0.03 & 0.07 & 1 & 0.04 & 0.25 & -0.02 & -0.16 & -0.08 & -0.01 & -0.09 & -0.03 & 0.00 & -0.05 & -0.12 & 0.44 \\
\hline SAP & 0.18 & 0.09 & -0.04 & 0.06 & 0.05 & 1 & 0.12 & 0.02 & 0.07 & -0.10 & 0.06 & -0.06 & 0.09 & 0.08 & 0.09 & 0.05 & 0.06 \\
\hline SY1 & 0.09 & 0.24 & -0.02 & 0.14 & 0.18 & 0.11 & 1 & 0.29 & -0.11 & 0.00 & 0.04 & -0.02 & -0.02 & 0.05 & 0.01 & -0.05 & 0.54 \\
\hline DI & 0.04 & 0.07 & 0.03 & 0.14 & 0.05 & 0.06 & 0.30 & 1 & 0.04 & -0.04 & 0.21 & 0.09 & -0.07 & 0.21 & 0.20 & 0.03 & 0.07 \\
\hline PLS & 0.12 & -0.02 & -0.06 & 0.05 & -0.12 & 0.04 & -0.06 & 0.05 & 1 & 0.03 & -0.07 & -0.14 & 0.20 & -0.09 & -0.05 & 0.17 & -0.14 \\
\hline HDL & -0.08 & -0.09 & -0.04 & 0.10 & -0.06 & -0.11 & -0.03 & -0.05 & 0.00 & 1 & -0.24 & -0.03 & -0.11 & -0.24 & -0.29 & 0.02 & 0.05 \\
\hline HGB & -0.11 & 0.00 & 0.30 & 0.04 & 0.06 & 0.08 & 0.07 & 0.15 & -0.05 & -0.18 & 1 & -0.01 & 0.03 & 0.97 & 0.78 & -0.14 & -0.07 \\
\hline LYP & -0.10 & 0.02 & 0.03 & 0.00 & -0.03 & -0.02 & -0.03 & 0.06 & -0.08 & -0.01 & -0.02 & 1 & -0.25 & 0.01 & 0.03 & -0.02 & -0.10 \\
\hline WBC & 0.15 & 0.04 & -0.02 & 0.03 & -0.04 & 0.07 & 0.00 & -0.04 & 0.14 & -0.10 & 0.06 & -0.26 & 1 & 0.02 & 0.02 & 0.23 & -0.06 \\
\hline HCT & -0.10 & 0.01 & 0.28 & 0.03 & 0.07 & 0.09 & 0.08 & 0.15 & -0.06 & -0.17 & 0.86 & -0.01 & 0.05 & 1 & 0.84 & -0.12 & -0.06 \\
\hline RBC & -0.07 & 0.05 & 0.25 & 0.02 & 0.04 & 0.11 & 0.05 & 0.14 & -0.03 & -0.20 & 0.63 & 0.01 & 0.05 & 0.68 & 1 & -0.03 & -0.17 \\
\hline PLT & 0.10 & 0.00 & -0.03 & 0.07 & -0.09 & 0.05 & -0.04 & 0.02 & 0.12 & 0.01 & -0.08 & 0.00 & 0.17 & -0.07 & -0.02 & 1 & -0.12 \\
\hline AGE & 0.13 & 0.35 & -0.15 & 0.19 & 0.31 & 0.06 & 0.38 & 0.11 & -0.08 & 0.02 & -0.04 & -0.07 & -0.04 & -0.03 & -0.10 & -0.08 & 1 \\
\hline
\end{tabular}


has higher correlation with some of the biomarker features (Systolic blood pressure, blood urea nitrogen, glycated hemoglobin) and low correlation with C-reactive protein, seram alkaline phosphatase, diastolic blood pressure, etc. Some biomarkers (e.g., pulse, red blood cell count and albumin) have negative correlation with age. Fig 4.8 (a) shows how two positively correlated biomarkers (systolic blood pressure $(\rho=0.54)$, blood urea nitrogen $(\rho=0.44)$ vary with age on average. Subjects in the NHANES dataset had ages in the range 18-85. Both mean systolic blood pressure and mean blood urea nitrogen increase consistently with age. Conversely, Fig 4.8 (b) shows how two negatively correlated biomarkers (albumin $(\rho=-0.17)$, red blood cell count $(\rho=-0.17)$, vary with age on average. Both mean albumin and red blood cell count decrease consistently with age. However, the variation of the decrease is not similar.


Figure 4.1: Variation of biomarkers with age. Features plotted for average measurements for individuals grouped by age (in years).

\subsubsection{Symbols/Notations Used}

The following notations are used in this paper: $T_{R}$ - training subjects, $T_{E}-$ test subjects, $C_{\mu}-$ trained centroids, $C_{m e^{-}}$trained medoids, $C_{\sigma^{-}}$trained standard deviation centroids, $C_{I Q R^{-}}$trained inter-quartile range medoids, $C$ - Centroid, $P_{i}-$ ith person, $B A_{i}-$ biological 
age of person $i, N$-number of neighbors, $E D$ - Euclidean distance vector, $\bar{C}_{P}$ - average age of selected N neighbors, $A_{C_{k}}$ - chronological age corresponding to the $C_{k}{ }^{\text {th }}$ centroid, $C_{\mu}^{S}$ - selected trained centroids, $\mu_{f}^{C}$ - the $f^{t h}$ feature of the $C^{t h}$ centroid of $C_{\mu}, \sigma_{f}^{C}$ - the $f^{t h}$ feature of the $C^{\text {th }}$ centroid of $C_{\sigma}, T_{R_{A}}$ - training data of the age $A$.

\subsubsection{Centroid BA: New Approach to Estimating BA}

We propose a new neighborhood-based method to predict biological age using biomarkers. Fig 4.2 shows the general structure of the proposed framework. Below we describe each component of the framework.

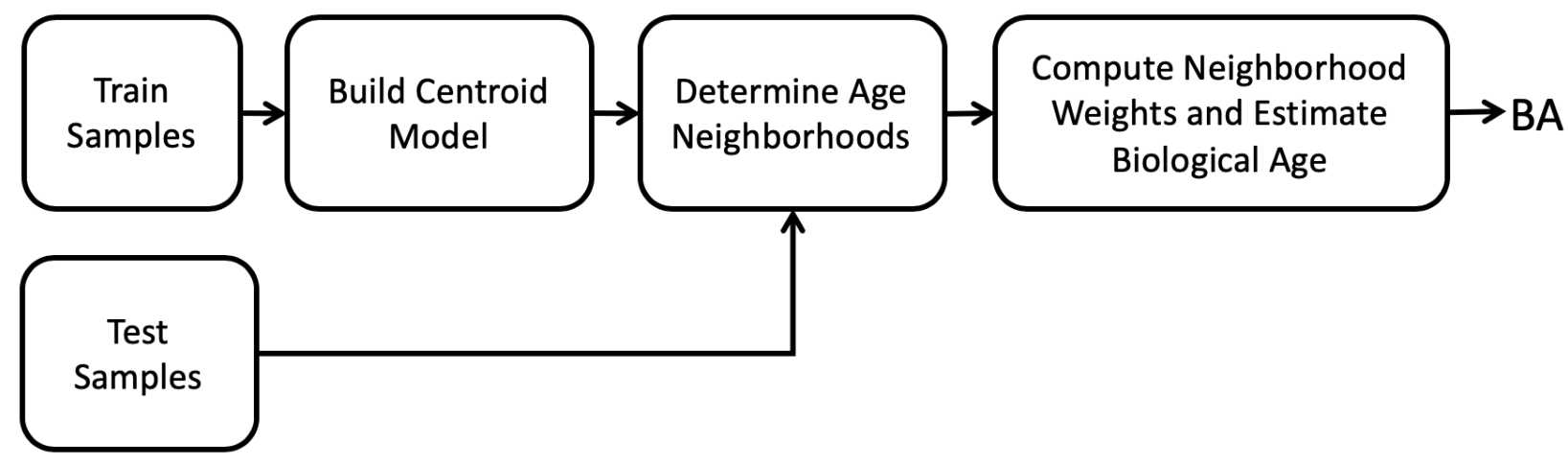

Figure 4.2: Proposed framework for centroid based biological age prediction.

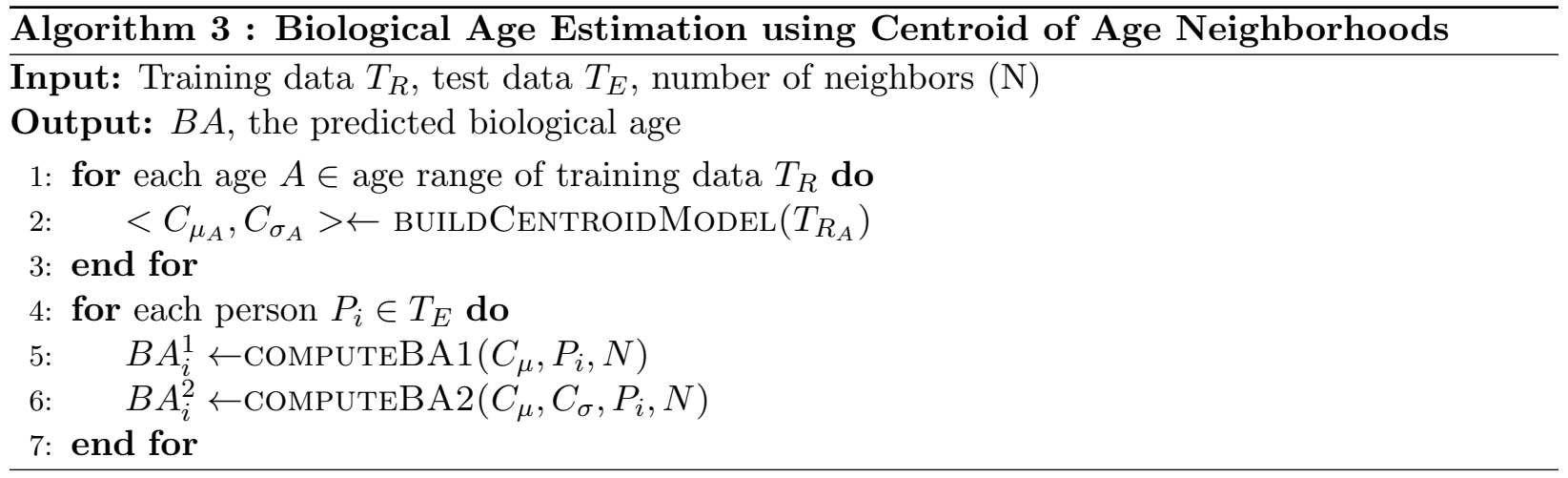

Each subject is represented using the biomarker attributes, forming a multidimensional feature space. Each subject is viewed as a point in the multidimensional feature space defined by the individual biomarker attributes (the features). Subjects in our NHANES dataset had 
ages in the range 18-85. During training, we calculate the centroid for each age. First, we compute the centroid based on the training dataset. We divide the dataset in 68 groups based on the chronological age. We have 68 centroids (age range 18-85 inclusive). Then we calculate the mean and standard deviation of each feature for all the age groups. We denote these as $C_{\mu}$ and $C_{\sigma}$, respectively. At the testing stage, given a person $P$ with an unknown biological age, we first determine the age neighborhoods for this individual $P$ based on person P's biomarker attributes and the precomputed age centroids at training. Then we estimate the BA using the age neighborhoods. Algorithm 3 shows the pseudo-code of the proposed centroid-based approach to biological age estimation. Clearly, how we determine the age neighborhoods is an important element in our proposed approach. Below, we describe two approaches to address this problem.

\section{Approach 1: Using only $C_{\mu}$.}

For a given person $P$, we first calculate and record the Euclidean distances from all the 68 centroids. Now, based on the sorted Euclidean distances, we select the required $N$ number of neighbors added with two centroids (these centroids are used later on for removing outliers). Then we compute $\bar{C}_{P}$, mean age of the selected centroids, and record the distances $\Delta_{j}$ of each selected neighbor from the $\bar{C}_{P}$. We consider two weighting schemes in computing the biological age.

a) Simple Average: This is the simplest approach, where based on the selected centroids we calculate the mean age, $\bar{C}_{P}$. Based on the distance from the mean age, we remove two outliers that are farthest from the mean. Now we calculate the average age of the remaining centroids.

b) Weighted exponential squared distance: First, we calculate the mean age $\left(\bar{C}_{P}\right)$. Now we compute the squared distances $\left(\Delta_{i}=\left(\bar{C}_{P}-C_{i}\right)^{2}\right)$ of each neighbor from the mean; we now calculate weight $w_{j}=\exp ^{-\Delta_{j}}$ and sum of weights $W=\sum_{j=1}^{N} w_{j}$. Finally, we calculate the 


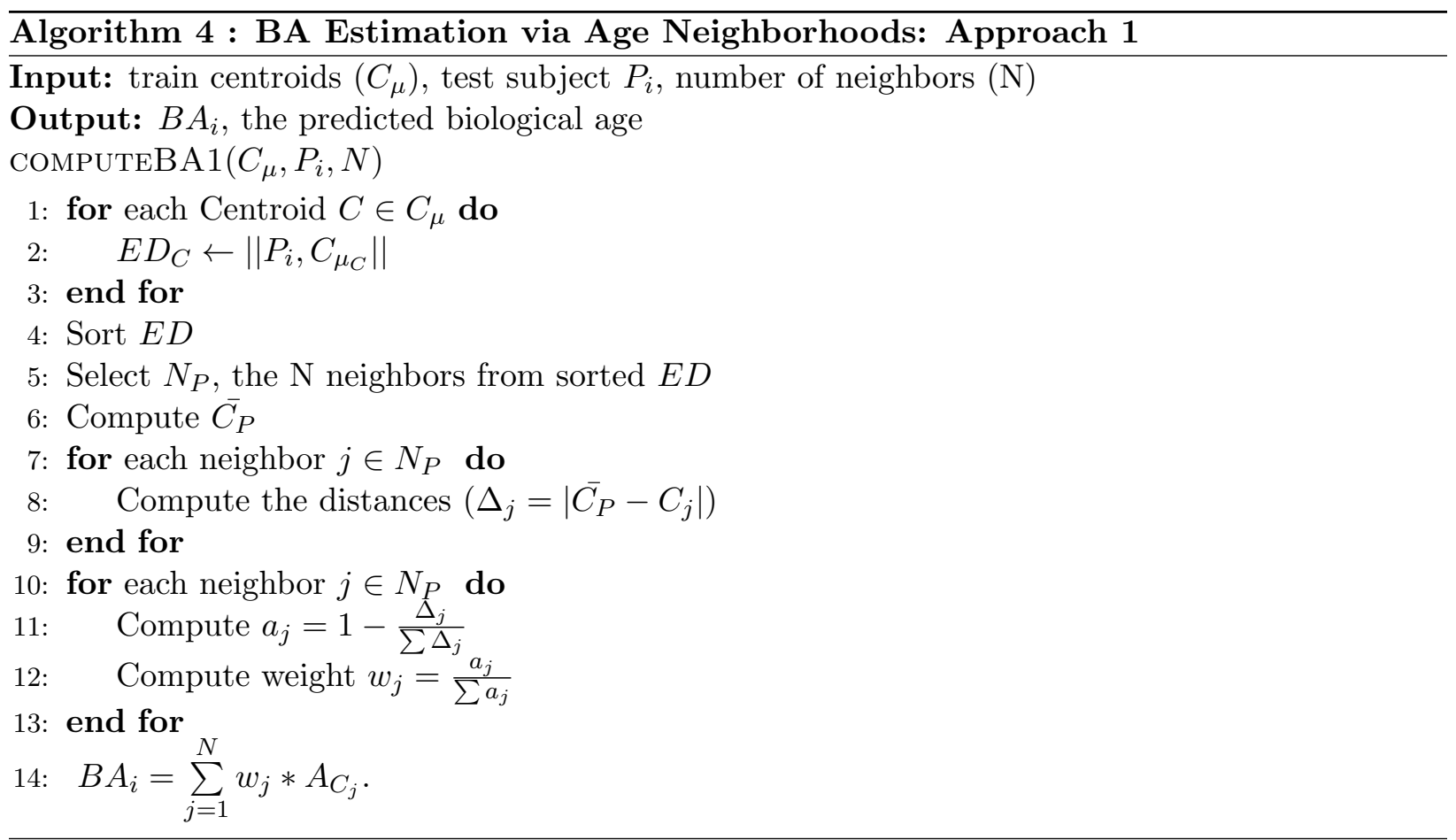

centroid BA. $B A_{i}=\sum_{j=1}^{N}\left(\frac{w_{j}}{W} * A_{C_{j}}\right)$. Algorithm 4 shows the pseudo-code summarizing the proposed approach.

\section{Approach 2: Using Both $C_{\mu}$ and $C_{\sigma}$.}

In the above basic approach, we used the centroid which is calculated based on the mean of the individual features. But the distributions of the features were ignored. To address this issue, in Approach 2, we incorporate the standard deviation (of each individual feature) along with their mean. Both $C_{\mu}$ and $C_{\sigma}$ are calculated based on the training dataset. We have 68 centroids and 68 standard deviations for each individual features, one pair for each age range between 18 to 85 . Given person $P$, we estimate the biological age based on these (feature centroid, dispersion) pairs. For a given person $P$, we first calculate and record the Euclidean distances from all the 68 trained centroids $C_{\mu}$. Now, based on the sorted Euclidean distances, we select a subset of centroid $C_{\mu}$ and the standard deviation $C_{\sigma}$. Then we apply two different parameters ( $\alpha$ and $\tau$-see below) to determine the neighbors that will 
be considered to calculate biological age. Based on the selected number of neighborhood centroids, the algorithm will use the new centroids that pass the thresholds. If not enough neighbors are found, the algorithm defaults to Approach 1. Algorithm 5 shows the pseudocode for this improved approach.

Two key parameters in the proposed approach are $\alpha$ and $\tau$. The first parameter $\alpha$ is a factor that we used to compute the lower range $(\mu-\alpha * \sigma)$ and higher range $(\mu+\alpha * \sigma)$ for evaluating similarity between corresponding features. The ranges are calculated for each feature individually. Essentially, $\alpha$ is used to restrict the allowed distance between the given feature for a subject say $P$, and the corresponding feature from the centroid for a given age category. With increasing values of $\alpha$, more distance is allowed, and hence leading to a less stringent criteria. Conversely, when $\alpha$ is small, only centroids that are very close to $P$, on the given feature will be involved in computing the BA for $P$. The parameter $\tau$ is a threshold count that is used to determine how many similar features ( $\%$ of the matches) are allowed in selecting a centroid. Here, even if one feature is found to be very close between $P$, and a given centroid, say $C$, this centroid may still not be used to estimate the BA for $P$ unless some other features are similar between $P$ and $C$, and the fraction of similar (or matching) features are above $\tau$, the threshold on the number of feature matches. With higher values of $\tau$, we have a more stringent criteria for selecting the thresholds.

Both $\alpha$ and $\tau$ can affect the performance of the proposed method. In our experiments, to find the best combination of $\alpha$ and $\tau$ in estimating the BA, we varied $\alpha$ in the range $\alpha=0.25$, $0.5,1.0,1.5,2.0$ and $\tau$ in the range $\tau=0.5,0.75,0.9$.

\subsubsection{Medoid BA: Estimating BA using Medoid of Age Neighborhoods}

Similar to the mean of each age category (the centroids), we also considered median representation of each age cluster. We call the estimated biological age (BA) based on median, as medoid-based BA. Algorithm 6 shows the pseudo-code of the proposed medoid-based 


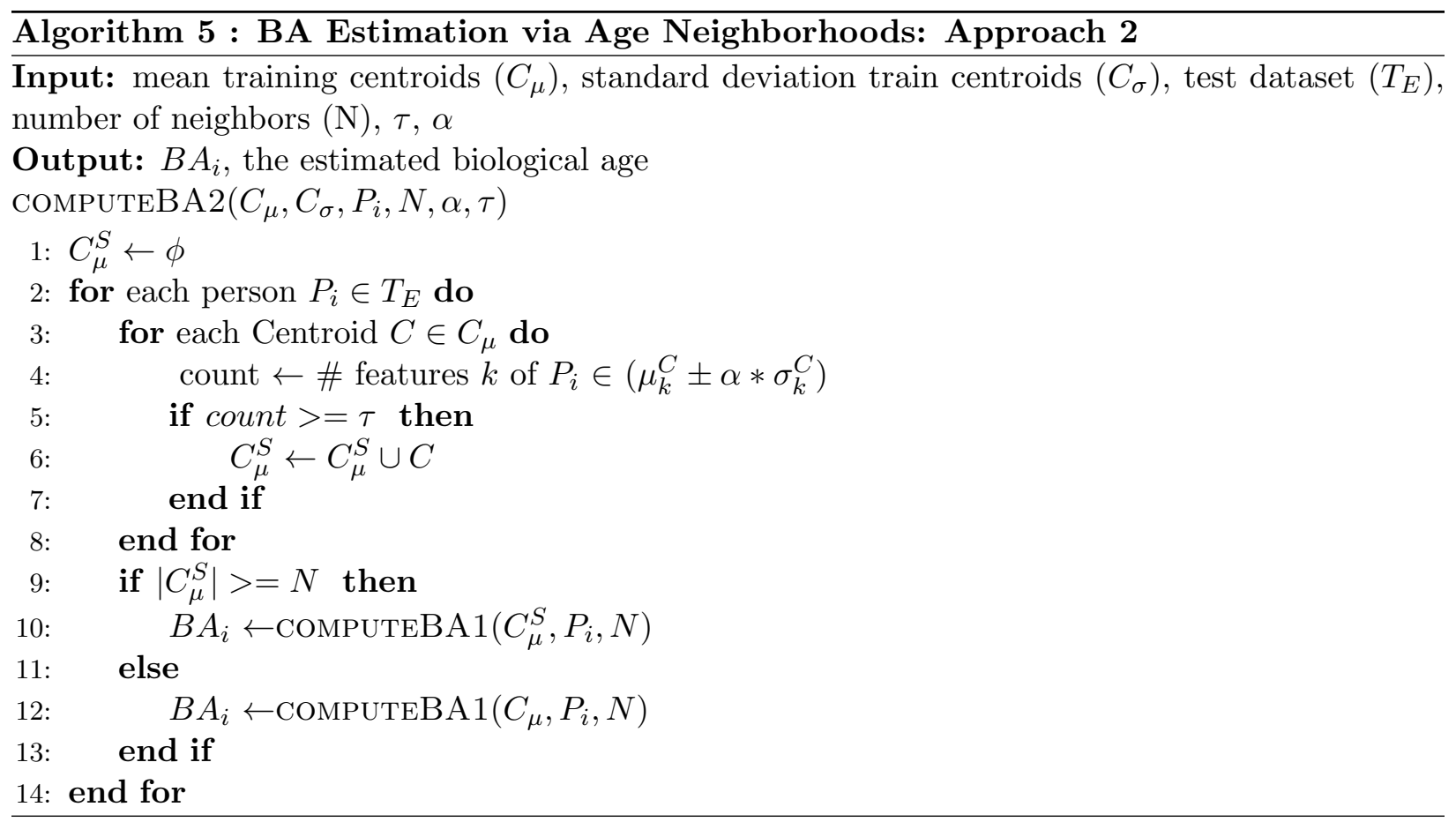

biological age estimation. Similar to Algorithm 3, for every age (in the range 18-85) we calculate a train medoid of the attributes, where the medoid is a vector in the feature space that contains the respective median of each attribute at a given chronological age. First, we compute medoid based on the training dataset. We divide the dataset in 68 groups based on the chronological age. We have 68 medoids (age range 18-85 inclusive). Now we calculate the median and the inter quartile range (IQR) of each feature for each age group. We denote them as $C_{m e}$ and $C_{I Q R}$, respectively. Follwoing the centroid approach, we can now estimate a person's biological age based on the trained medoids and the recorded IQRs. The medoid approach is summarized in Algorithm 6. Algorithm 3 and Algorithm 6 are similar. The differences are, $C_{\mu}$ is replaced by $C_{m e}$, and $C_{\sigma}$ is replaced by $C_{I Q R}$. So, for medoid BA, to compute COMPUtEBA1(), we then use $C_{m e}$ and similarly for COMPUTEBA2(), we use both $C_{m e}$ and $C_{I Q R}$. 


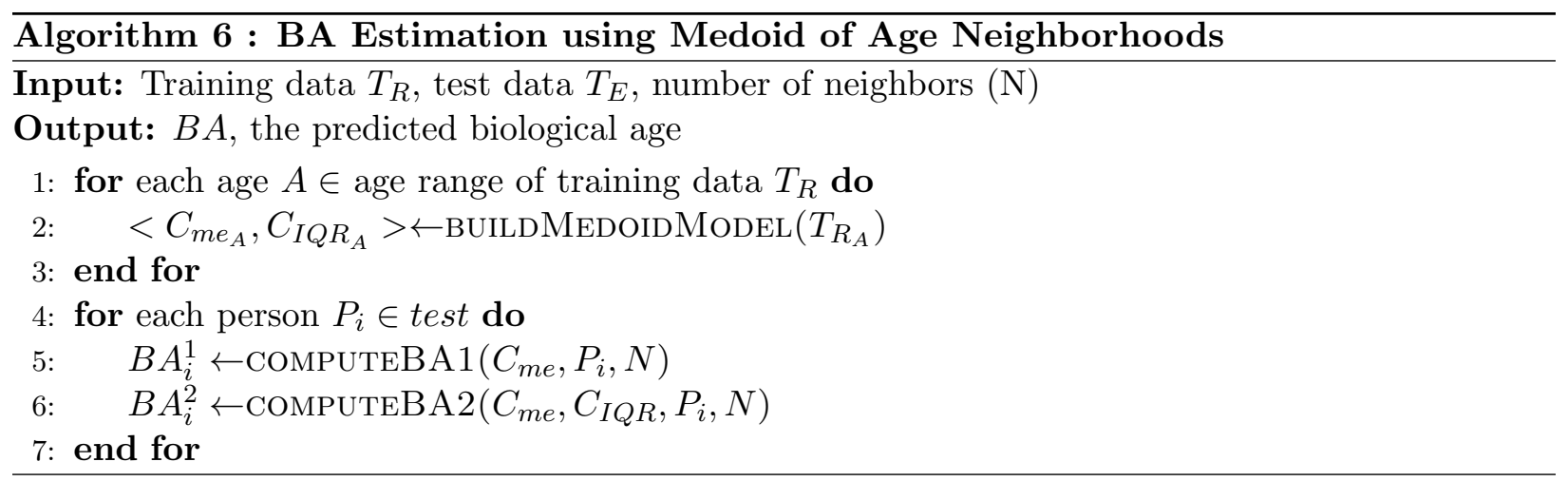

\subsection{Results}

For validation and comparison of the proposed BA algorithms, we have applied three statistical analysis methods, namely, Cox proportionality hazard (Cox PH) model, Kaplan-Meier (KM) curves, and survival area under the curve (AUC) of receiver operating characteristic (ROC). We randomly partitioned the dataset into training set and test set, using $2 / 3$ for training, the remaining $1 / 3$ for testing. All statistical analyses were performed using the $\mathrm{R}$ Language, Ver. 3.3.5 (The R Foundation for Statistical Computing, Vienna, Austria). The following packages were used: survival, gtools, ggplot2, tidyverse, keras, e1071, matrixStats, SurvAUC.

\subsubsection{Cox PH model.}

We used Cox proportionality mortality hazard modeling [39,84 to quantify the association of the proposed centroid BA or medoid BA with all-cause mortality. Under the Cox model, the relationship between hazard and the covariates is described by considering the logarithm of the hazard as a linear function of the variables. We have considered five BA estimation algorithms to calculate the hazard ratio (HR) [39]. First, we estimate BA using MLR method [89], KD method [83], DNN (Deep Neural Networks) [114], and our proposed models. For DNN, we used the network reported by Putin et al had the best performance. Then we calculate $\Delta=C A-B A$ for each BA estimation algorithm. We then use the $\Delta$ quartiles 
to apply the Cox model. Table 4.3 shows the results for the biomarker features. First, we applied chronological age in the Cox model. We observe that for MLR, KD, and DNN, HR value is $0.97,0.99$ and 0.97 respectively. And our proposed centroid-based and medoid-based methods have similar results (0.99 and 0.98 respectively). To further investigate, we use the estimated BA as the parameter for the Cox model. Interestingly, the HR increased for all the methods (Centroid 1.12, Medoid 1.12, MLR 0.99, KD 1.04, and DNN 1.12). From the perspective of Cox PH model, we found that proposed centroid-based and medoid-based BA estimation methods had similar or slightly better performance than the other methods.

Table 4.3: Cox PH results for biological age estimation methods.

\begin{tabular}{|l|c|c|c|c|}
\hline & \multicolumn{2}{|c|}{ Chronological Age } & \multicolumn{2}{c|}{ Biological Age } \\
\hline & HR & p-value & HR & p-value \\
\hline MLR & 0.97 & 0.00 & 0.99 & 0.00 \\
\hline KD & 0.99 & 0.00 & 1.04 & 0.00 \\
\hline DNN & 0.97 & 0.00 & 1.12 & 0.00 \\
\hline Centroid & 0.99 & 0.00 & 1.12 & 0.00 \\
\hline Medoid & 0.98 & 0.00 & 1.12 & 0.00 \\
\hline
\end{tabular}

\subsubsection{KM Curves and Log-rank test}

To further study the performance of centroid BA, we analysed the Kaplan-Meier (KM) survival curves [80] obtained using the quartiles of delta $(\Delta=C A-B A)$. Fig. 4.3 shows the KM plots using the estimated BA from the proposed Centroid approach. Fig. 4.4 shows the results for the proposed Medoid approach. In general, biological age performs well in distinguishing the proportion of survivors for each method. From the figure, it is apparent that when applied in survival model, using the estimated biological age from each method seemed to perform better than using chronological age. Among the methods, the proposed centroid and medoid based approaches have similar survival curves. To further quantify the performance, we used the log-rank test to compare the survival distributions obtained using the different BA algorithms. The log-rank test can be used to compare different KaplanMeier curves to see if they are statistically equivalent. The output of the test is a $\chi^{2}$-distance, 
and the p-value associated with the distance. Higher $\chi^{2}$-distances and low p-values indicate a better separation between the curves, and hence a better performance in mortality modeling. The differences among the biological ages estimated by the four methods are more evident using quantitative measures, e.g., the $\chi^{2}$-distance between their respective KM curves, as captured by the logrank test (Table 4.4). Proposed medoid based approach has the best overall results using either chronological age, or biological age.

Table 4.4: Log Rank results ( $\chi^{2}$-distance) for mortality modeling using four biological age prediction methods applied to the blood biomarkers.

\begin{tabular}{|l|c|c|c|c|}
\hline & \multicolumn{3}{|c|}{ Biomarker } \\
\hline & \multicolumn{2}{|c|}{ Age } & \multicolumn{2}{c|}{ Estimated BA } \\
\hline & Chi-Sq & p-value & Chi-Sq & p-value \\
\hline MLR & 74.24 & 0.00 & 32.75 & 0.00 \\
\hline KD & 22.68 & 0.00 & 157.59 & 0.00 \\
\hline DNN & 74.25 & 0.00 & 439.83 & 0.00 \\
\hline Centroid & 81.51 & 0.00 & 689.37 & 0.00 \\
\hline Medoid & 115.15 & 0.00 & 707.42 & 0.00 \\
\hline
\end{tabular}

Table 4.5: Area under the curve (AUC) of receiver operating characteristics (ROC) curves.

\begin{tabular}{|l|c|c|}
\hline & Delta & Estimated BA \\
\hline MLR & 0.60 & 0.64 \\
\hline KD & 0.57 & 0.60 \\
\hline DNN & 0.65 & 0.51 \\
\hline Centroid & 0.61 & 0.64 \\
\hline Medoid & 0.66 & 0.66 \\
\hline
\end{tabular}

\subsubsection{ROC of $\mathrm{AUC}$}

We have used the receiver operating characteristics (ROC) curves to examine the sensitivity and specificity of chronological age and the predicted biological ages in mortality modeling. We have applied estimators of cumulative and incident/dynamic area under curve (AUC) proposed by Song and Zhou [132]. These estimators are given by the areas under the time dependent ROC curves estimated by sensitivity and specificity. Fig. 4.5 shows the estimated ROC curves for the four biological age prediction methods. Using the ROC curve on $\Delta=$ 


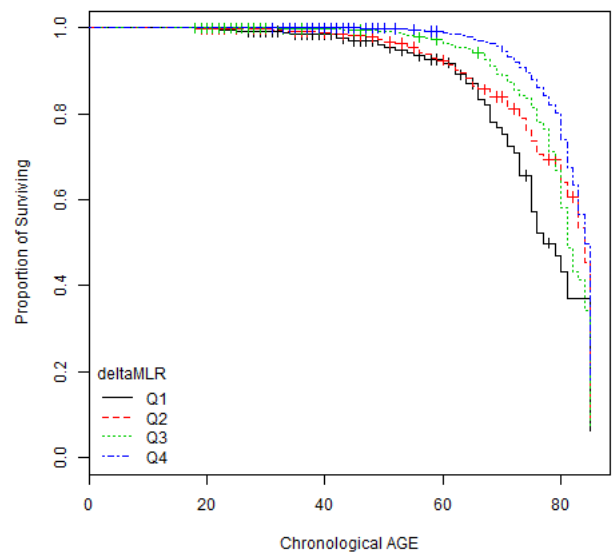

(a) QdeltaMLR

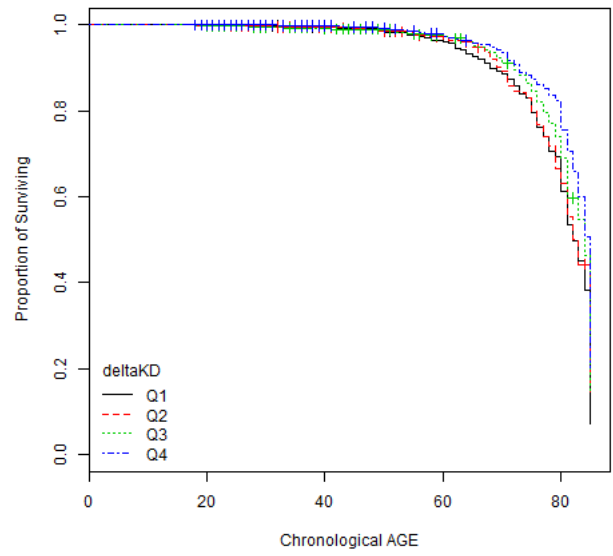

(c) QdeltaKD

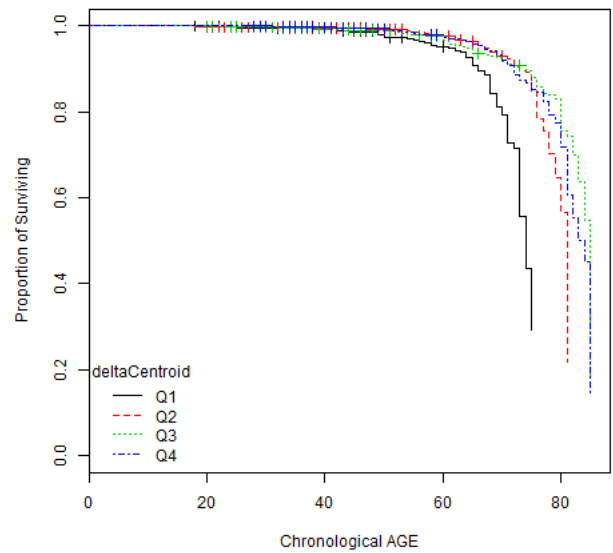

(e) QdeltaCentroid

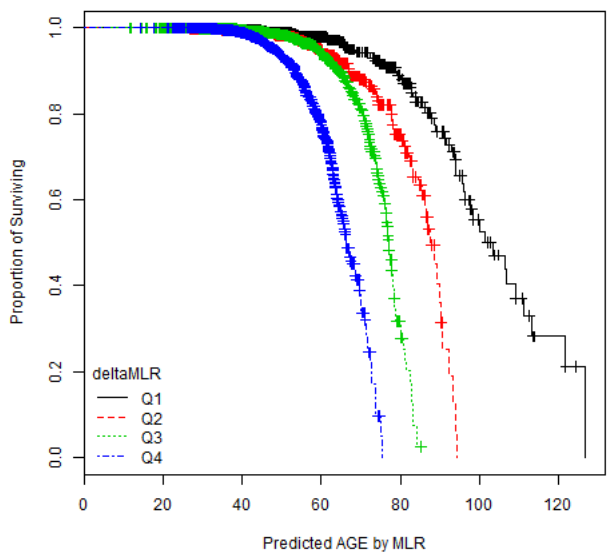

(b) Qdelta $M L R_{B A}$

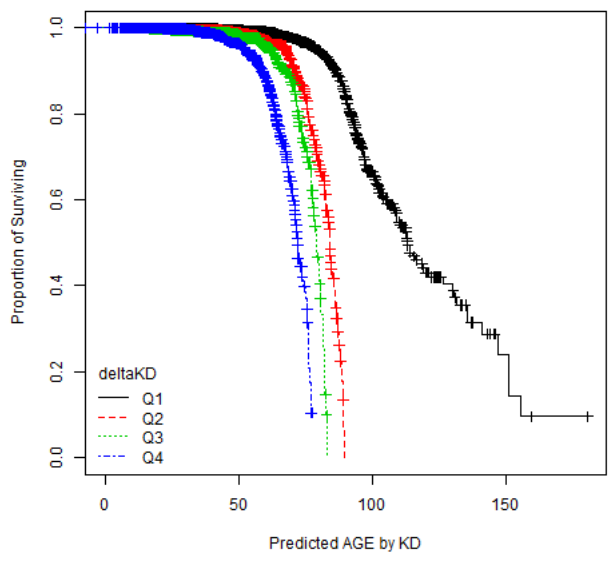

(d) QdeltaK $D_{B A}$

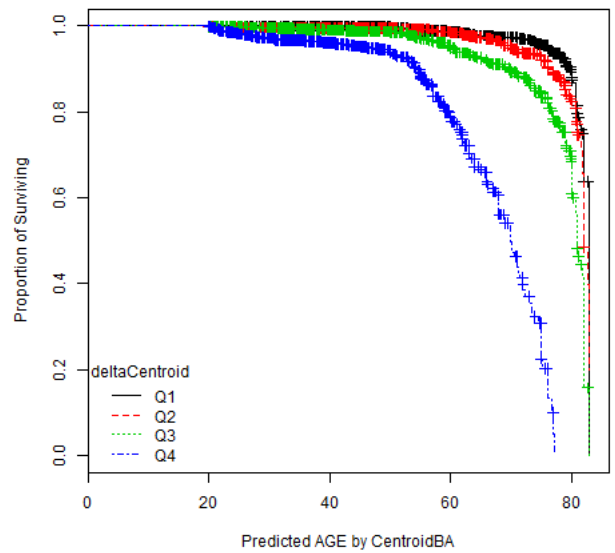

(f) QdeltaCentroid $B A$

Figure 4.3: The Kaplan Meier curves for three BA estimation algorithms using biomarker features. Results are reported for (a, c, e) chronological age, and (b, d, f) predicted BA. Q1, Q2, etc. denote 1st quartile, 2nd quartile, etc. 


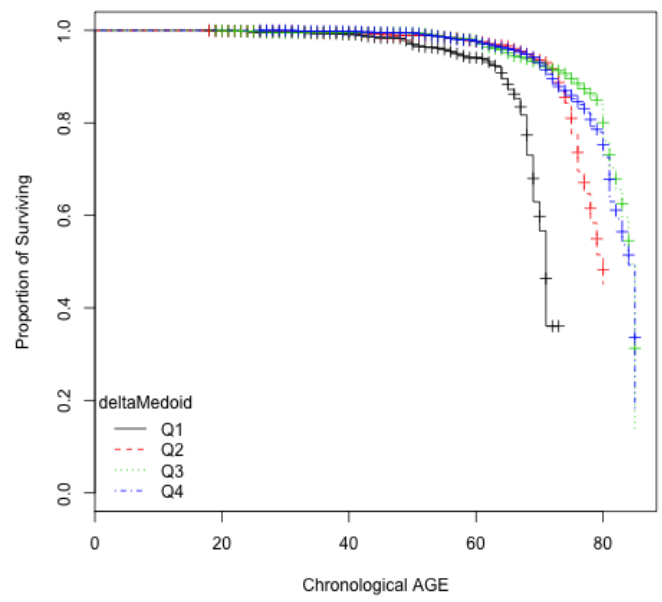

(a) QdeltaMedoid

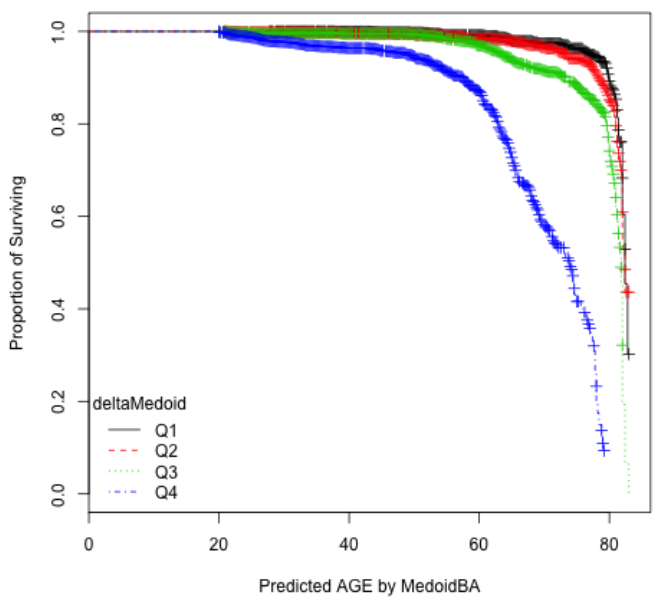

(b) QdeltaMedoid $B A$

Figure 4.4: The Kaplan Meier curves for Medoid using biomarker features (a) chronological age, and (b) predicted BA. Q1, Q2, etc. denote 1st quartile, 2nd quartile, etc.

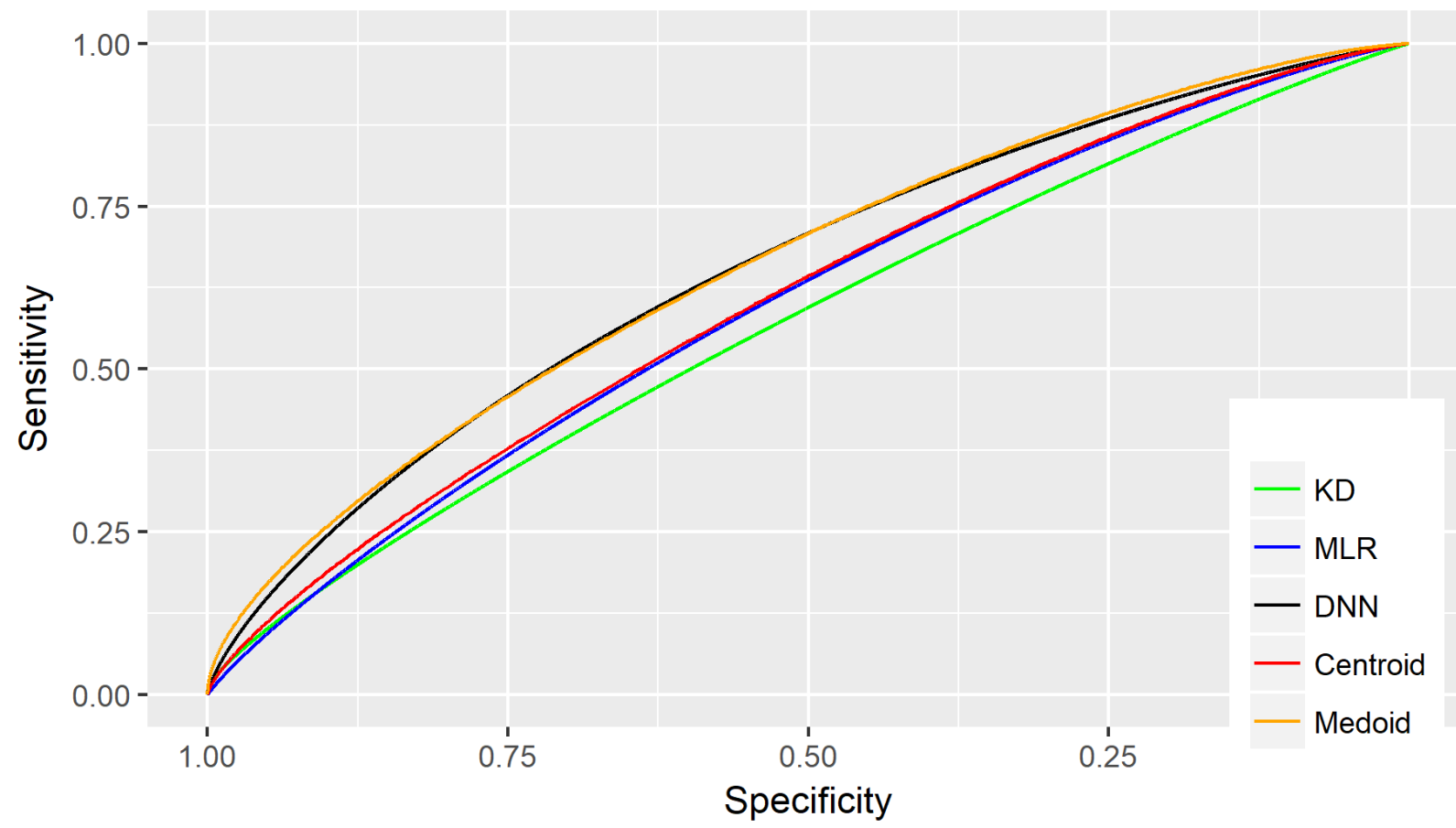

Figure 4.5: ROC curves for MLR, KD, DNN, Centroid, and Medoid algorithms using biomarker features. Results are reported for applying $\Delta=C A-B A$ for the respective estimation approach. 
$C A-B A$, the best performing biological age estimate was the proposed medoid BA (AUC $=0.66)$ followed by centroid $(\mathrm{AUC}=0.61)$. MLR, KD, and DNN have AUC values of 0.60, 0.57 , and 0.65 , respectively. The results improved using the estimated BA rather than $\Delta$. Observing the results on the biomarker features, the proposed medoid and centroid based BA estimation algorithms produced the best results with respect to the area under the curve of ROC. Cox model performance in terms of hazard ratio (HR) is similar in comparison to MLR, $\mathrm{KD}$, or DNN approaches. Using the KM curves and log-rank test on the results from the proposed centroid-based and medoid-based approaches resulted in improved performances over the previous approaches. These results suggest that the centroid based model is a competitive BA predictor. The medoid-based model showed an improvement over all the other methods.

\subsubsection{Results for Approach 2}

Applying the proposed Approach 2 (Algorithm 5) using both the $C_{\mu}, C_{\sigma}$ improved the results. Table 4.6 shows the results for applying log-rank test, and AUC of ROC survival curves for variation of different values of the parameter $\alpha$ and $\tau$. We varied parameter $\alpha$ ( $\alpha=0.25,0.5,1,1.5,2)$ and $\tau(\tau=0.5,0.75,0.9)$ to test the performance of the proposed approach. Parameter $\alpha$ is varied to test the impact of the range allowed, and parameter $\tau$ is varied to check the impact of percentage of feature matches. We have considered 15 possible variations of $\alpha$ and $\tau$ for these sets of values. As mentioned earlier, applying $\alpha$ and $\tau$ is a more robust approach. We notice that the above two mentioned criteria ( $\chi^{2}$-distance using the log-rank test, survival AUC) improved for the centroid method using Approach2. Although for parameter $\alpha=0.25$, and $\tau=0.5$ the $\chi^{2}$-distance is highest, but the range is too small. Thus we chose $\alpha=0.5$, and $\tau=0.5$ as our best combination. We observed that survival AUC improved from 0.61 (Table 4.5) to $0.75, \chi^{2}$ distance of the log-rank test increased from 689.37 to 767.75, and the hazard ratio of Cox PH model also improved from 1.120 (Table 4.3) to 1.128 . 

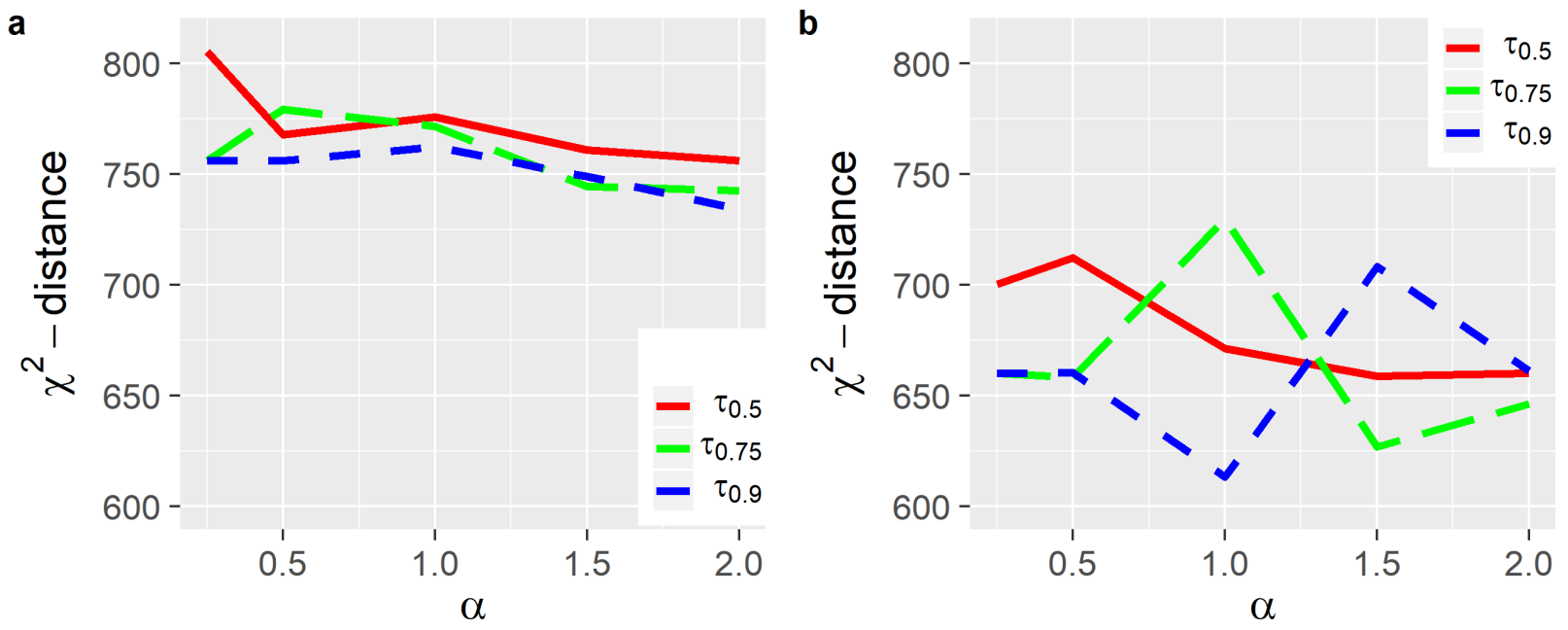

Figure 4.6: Impact of parameter $\alpha$ and $\tau$ in Approach2 (a) using Centroid BA, and (b) using Medoid BA.

Fig. 4.6 shows the impact of $\alpha$, and $\tau$ parameters on $\chi^{2}$-distance of the log-rank test using Approach 2 for both the centroid and medoid methods.

Table 4.6: Results for varying $\alpha$ and $\tau$ in Approach2 using centroid BA (algorithm 5), for $\mathrm{N}=5$.

\begin{tabular}{|c|c|c|c|c|c|c|}
\hline & \multicolumn{2}{|c|}{$\tau=0.5$} & \multicolumn{2}{c|}{$\tau=0.75$} & \multicolumn{2}{c|}{$\tau=0.90$} \\
\hline$\alpha$ & $\chi^{2}$-dist & AUC & $\chi^{2}$-dist & AUC & $\chi^{2}$-dist & AUC \\
\hline 0.25 & $\mathbf{8 0 5 . 2 7}$ & 0.74 & 755.98 & 0.73 & 755.98 & 0.73 \\
\hline 0.5 & 767.75 & $\mathbf{0 . 7 5}$ & 779.3 & 0.74 & 756.01 & 0.73 \\
\hline 1 & 775.69 & 0.74 & 771.6 & 0.74 & 762.19 & 0.74 \\
\hline 1.5 & 760.79 & 0.74 & 744.28 & 0.74 & 748.88 & $\mathbf{0 . 7 5}$ \\
\hline 2 & 755.98 & 0.73 & 742.55 & 0.74 & 733.95 & 0.74 \\
\hline
\end{tabular}

All the corresponding $\mathrm{p}$-values are significant $(p \approx 0)$ for log-rank test.

Similarly, Table 4.7 shows the results of varying $\alpha$ and $\tau$ for medoid BA using Approach 2. We observe that survival AUC value improved from 0.66 (Table 4.5 to $0.75, \chi^{2}$ distance of the log-rank test increased from 707.42 to 712.10, and the hazard ratio of Cox PH model remained similar (1.12) (using $\alpha=0.5$ and $\tau=0.5$ ). For both the centroid approach and the medoid approach, our observation is that parameter settings $(\alpha=0.5, \tau=0.5)$ usually leads to he best result, or close to the best. Thus we have this setting in comparative analysis of the methods. 
Table 4.7: Results for varying $\alpha$ and $\tau$ in Approach2 using medoid BA (algorithm 6), for $\mathrm{N}=5$.

\begin{tabular}{|c|c|c|c|c|c|c|}
\hline & \multicolumn{2}{|c|}{$\tau=0.5$} & \multicolumn{2}{c|}{$\tau=0.75$} & \multicolumn{2}{c|}{$\tau=0.90$} \\
\hline$\alpha$ & $\chi^{2}$-dist & AUC & $\chi^{2}$-dist & AUC & $\chi^{2}$-dist & AUC \\
\hline 0.25 & 700.20 & 0.75 & 660.17 & 0.75 & 660.16 & 0.75 \\
\hline 0.5 & $\mathbf{7 1 2 . 1 0}$ & $\mathbf{0 . 7 6}$ & 658.22 & 0.75 & 660.22 & 0.75 \\
\hline 1 & 671.20 & 0.75 & $\mathbf{7 2 9 . 6 5}$ & 0.75 & 613.19 & 0.75 \\
\hline 1.5 & 658.70 & 0.75 & 626.75 & 0.75 & 708.25 & 0.76 \\
\hline 2 & 660.20 & 0.75 & 646.18 & 0.75 & 661.25 & 0.75 \\
\hline
\end{tabular}

All the corresponding $\mathrm{p}$-values are significant $(p \approx 0)$ for log-rank test.

\subsubsection{Comparative Results}

To place the results of our proposed approaches in perspective, we have compared with current popular approaches to BA estimation using biomarker data, namely KD [83], MLR [89], and DNN [114]. We have shown the corresponding results with these methods while we discuss the results of our proposed approaches (see Tables 4.3, 4.4, and 4.5)

Table 4.8 shows the comparative performance of existing popular methods and the proposed approaches. The results show that our proposed Approach 2 using both Centroid2 $\left(C_{\mu}, C_{\sigma}\right)$ and Medoid2 $\left(C_{m e}, C_{I Q R}\right)$ have the best overall results. The results for Approach 2 are reported for using $\alpha=0.5$ and $\tau=0.5$.

Table 4.8: Overall comparative results on biological age prediction.

\begin{tabular}{|c|c|c|c|c|c|}
\hline & \multicolumn{2}{|c|}{$\mathrm{CoxPH}$} & \multicolumn{2}{|c|}{ Log-rank } & AUC \\
\hline & $\mathrm{HR}$ & p-value & $\chi^{2}$-dist & p-value & \\
\hline MLR 89 & 0.99 & 0 & 32.75 & 0 & 0.60 \\
\hline $\mathrm{KD} 83$ & 1.04 & 0 & 157.59 & 0 & 0.57 \\
\hline DNN $[114$ & 1.12 & 0 & 439.83 & 0 & 0.65 \\
\hline Centroid & 1.12 & 0 & 689.37 & 0 & 0.61 \\
\hline Centroid2 & 1.13 & 0 & 767.75 & 0 & 0.75 \\
\hline Medoid & 1.12 & 0 & 707.42 & 0 & 0.66 \\
\hline Medoid2 & 1.12 & 0 & 712.1 & 0 & 0.76 \\
\hline
\end{tabular}




\subsection{Discussion}

\subsubsection{Impact of Number of Neighbors.}

Two key steps in the proposed method are how to select the neighbors, and how to determine the number of neighbors to be involved. Algorithm 4 and Algorithm 5 have shown how the neighbors are selected, once we know the number of neighbors. Given the significance of neighborhoods in our approach, it becomes important to study how the neighborhood size can influence our results. To check the impact of neighborhood size on our proposed centroid of age neighborhoods for biological age estimation, we experimented with different values for $\mathrm{N}$, the number of neighbors. We have considered 10 values of $N$, namely, $N=1,2,3,5,7$, $10,20,30,40,50$. We show the impact of $N$ based on the KM plots and the corresponding log-rank test. We also studied changes in error density with increasing $\mathrm{N}$.

Table 4.9 shows the results of log-rank test using both estimated BA and delta $(\Delta=C A-$ $B A)$ for different number of neighbors. Based on the $\chi^{2}$ distance, we observe that BA estimated using 10 neighbors has the maximum value followed by 5 neighbors. When using delta, best results were obtained with $N=2$, followed by $N=5$. Table 4.9 also shows how mean absolute error (MAE) vary with increasing number of neighbors in our proposed centroid-based method. The MAE starts high $(N=1)$, then it reduces sharply at $N=2$. Considering just a single neighbor leads to a significant error in the estimation. The sharp decrease at $N=2$ is understandable because it averaged out the error introduced by the single neighbor. MAE is similar for $N=2$ to $N=20$. After that the MAE starts to increase almost linearly with the increasing $N . N=20$ has the overall lowest MAE. The performance

criteria for choosing $N$ was to get lower MAE, and higher $\chi^{2}$-distance using both estimated BA and delta $(\Delta=C A-B A)$. From these results, we selected $N=5$ as the overall best number of neighbors. 
Table 4.9: Impact of number of neighbors on mean absolute error (MAE), log-rank tests on estimated biological age, delta (CA-BA).

\begin{tabular}{|c|c|c|c|c|c|}
\hline & MAE & \multicolumn{2}{|c|}{ Estimated BA } & \multicolumn{2}{c|}{ delta (CA - BA) } \\
\hline $\mathrm{N}$ & & $\chi^{2}$-dist & p-val & $\chi^{2}$-dist & p-val \\
\hline 1 & 26.82 & $\mathbf{5 6 6 . 6 0}$ & 0 & $\mathbf{9 3 5 . 1 4}$ & 0 \\
\hline 2 & 11.51 & 399.8 & 0 & 723.7 & 0 \\
\hline 3 & 11.47 & 376.3 & 0 & 653.8 & 0 \\
\hline 5 & 11.35 & 491.2 & 0 & 703.9 & 0 \\
\hline 7 & 11.17 & 480.9 & 0 & 664.6 & 0 \\
\hline 10 & 10.92 & 506.1 & 0 & 562.1 & 0 \\
\hline 20 & $\mathbf{1 0 . 7 4}$ & 476.5 & 0 & 320.4 & 0 \\
\hline 30 & 11.2 & 494.6 & 0 & 154.6 & 0 \\
\hline 40 & 12.36 & 419.6 & 0 & 108.4 & 0 \\
\hline 50 & 14.02 & 394.1 & 0 & 84.0 & 0 \\
\hline
\end{tabular}

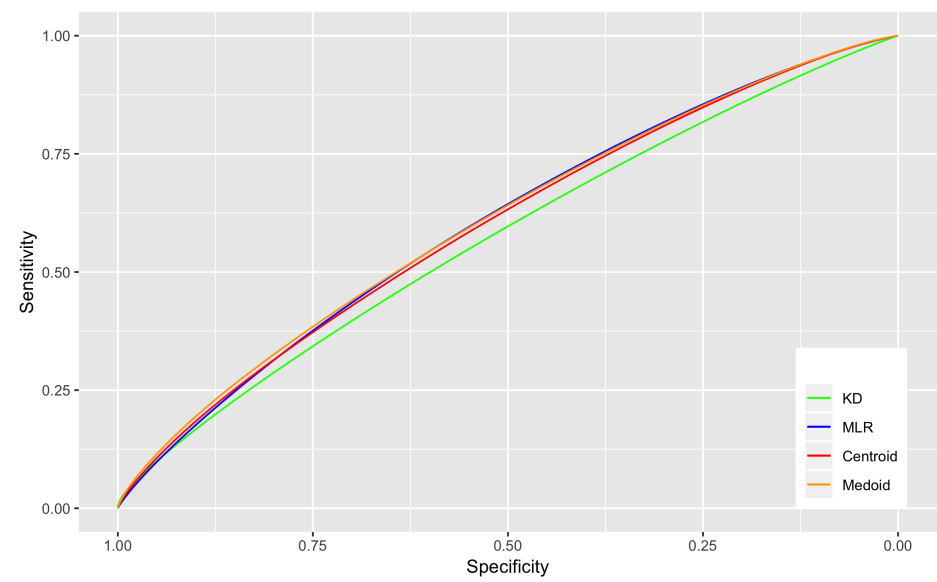

(a) Female

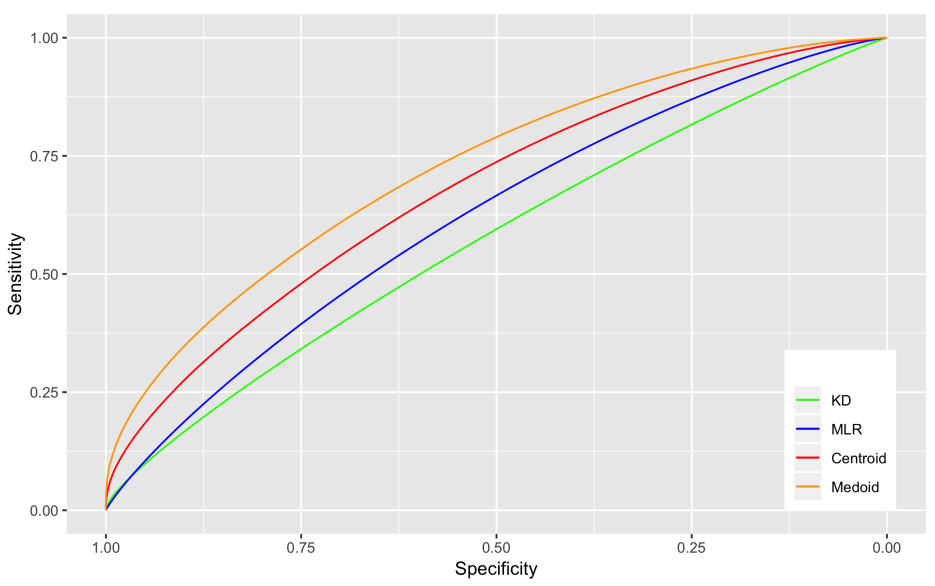

(b) Male

Figure 4.7: ROC curves for 4 biological age estimation algorithms using a)Female, and b)Male models separately on biomarker features. 


\subsubsection{Impact of Gender.}

Gender is expected to have some influence on the performance of an age estimation scheme [65]. Thus, most studies on age estimation have applied different models for female and male [54,62]. We have grouped the dataset into male and female subjects. Then we computed the centroids separately for both male and female. Fig. 4.7 shows the ROC curves using survival models. In Table 4.10 we show the AUC for each BA estimation algorithm for both female and male. The proposed medoid based method has the overall best result. For biomarker features, MLR has better results than both KD and the proposed centroid-based method. We also observe that, in every case independent of the particular method applied, or the specific features used, better results were obtained for male subjects than for female subjects. These results are consistent with other existing work that show that predicting age for female subjects is generally more difficult than for male subjects [54,62,65].

Table 4.10: Area under curve (AUC) of receiver operating characteristics (ROC) curves for separate male, female models.

\begin{tabular}{|l|c|c|c|c|}
\hline & \multicolumn{2}{|c|}{ Delta } & \multicolumn{2}{c|}{ Estimated BA } \\
\hline & Female & Male & Female & Male \\
\hline MLR & 0.60 & 0.62 & 0.64 & 0.63 \\
\hline KD & 0.57 & 0.57 & 0.63 & 0.58 \\
\hline Centroid & 0.60 & 0.68 & 0.64 & 0.62 \\
\hline Medoid & 0.61 & 0.72 & 0.66 & 0.63 \\
\hline
\end{tabular}

\subsection{Centroid-Based Age Neighborhood using Body Shape}

Fig 4.8 (a) shows how a positively correlated biomarker (systolic blood pressure ( $\rho=0.54$ ), and body shape index SBSI $(\rho=0.44)$ vary with age on average. Subjects in the NHANES dataset had ages in the range 18-85. Both mean systolic blood pressure and mean SBSI increase consistently with age. Conversely, Fig 4.8 (b) shows how a negatively correlated biomarker (hemoglobin $(\rho=-0.07$ ), and BMI $(\rho=0.16)$ vary with age on average. Hemoglobin is generally consistent within the age range 18-50 then decrease with age on average whereas 


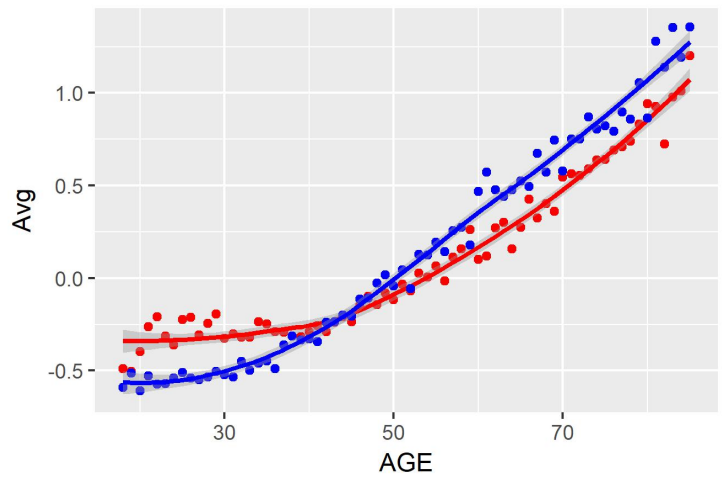

(a) SBSIvsBP

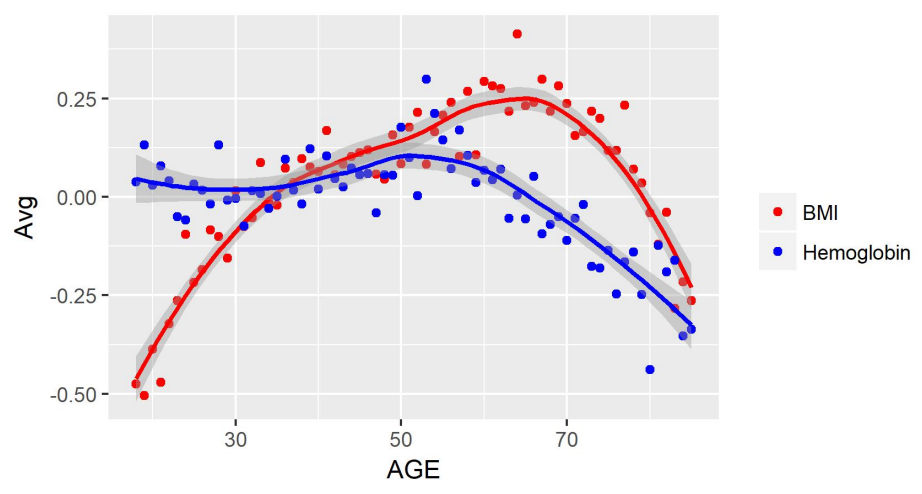

(b) BMIvsHGBN

Figure 4.8: Variation of different anthropometric and biomarkers attributes with age. Features plotted for average measurements for individuals grouped by age (in years).

the BMI goes up on average but after around age 60 goes down consistently.

Centroid-based approaches can be generalized to perform BA estimation on other features different from biomarkers (e.g. anthropometric features). Combination of features could also be used to estimate BA under this generalized framework.

Table 4.11 shows the results (Biomarker features). First, we applied chronological age in the Cox model. We observe that for MLR, KD, and DNN, HR value is 0.97, 0.99 and 0.97 respectively. And our proposed centroid-based method have similar results (0.99). To further investigate, we use the estimated BA as the parameter for the Cox model. Interestingly, the HR increased for all the methods (Centroid 1.12, MLR 0.99, KD 1.04, and DNN 1.12). Proposed centroid-based BA estimation methods had similar or slightly better performance than the other methods.

\subsubsection{KM Curves and Log-rank test}

To further study the performance of centroid BA, we analysed the Kaplan-Meier (KM) survival curves [80] obtained using the quartiles of delta $(\Delta=C A-B A)$. Fig. 4.9 shows the KM plots using the estimated BA from the proposed Centroid approach. To quantify 
Table 4.11: Cox PH results for biological age estimation methods.

\begin{tabular}{|c|c|c|c|c|c|c|}
\hline & \multicolumn{2}{|c|}{ Biomarkers } & \multicolumn{2}{c|}{ Anthropometric } & \multicolumn{2}{c|}{ Combined } \\
\hline & CA & BA & CA & BA & CA & BA \\
\hline MLR 89 & 0.97 & 0.99 & 0.98 & 1.13 & 0.98 & 1.15 \\
\hline KD 83 & 0.99 & 1.04 & 1.00 & 1.11 & 0.99 & 1.16 \\
\hline DNN & 0.97 & 1.12 & 0.73 & 0.90 & 0.53 & 0.69 \\
\hline Centroid1 & 0.99 & 1.12 & 0.99 & 1.06 & 0.98 & 1.12 \\
\hline Centroid2 & 0.99 & 1.13 & 0.99 & 1.06 & 0.99 & 1.13 \\
\hline Medoid1 & 0.98 & 1.12 & 0.99 & 1.12 & 0.98 & 1.12 \\
\hline Medoid2 & 0.98 & 1.12 & 0.99 & 1.13 & 0.99 & 1.13 \\
\hline
\end{tabular}

Corresponding p-values are significant. Results are reported as hazard ratio.

the performance, we used the log-rank test to compare the survival distributions obtained using the different BA algorithms. The log-rank test can be used to compare different Kaplan-Meier curves to see if they are statistically equivalent. The output of the test is a $\chi^{2}$-distance. Higher $\chi^{2}$-distances indicate a better separation between the curves, and hence a better performance. In general, the estimated biological age (BA) produced overall better results when compared with using the direct chronological age. This is in-line with observations by Levine [89] and Mitnitski et al [97]. The differences among the biological ages estimated by the methods are more evident using quantitative measures, e.g., the $\chi^{2}$ distance between their respective KM curves, as captured by the logrank test (Table 4.12). Proposed centroid based approach has the best overall results using either chronological age, or biological age for biomarker features.

Table 4.12: Log Rank results for biological age estimation methods.

\begin{tabular}{|c|c|c|c|c|c|c|}
\hline & \multicolumn{2}{|c|}{ Biomarkers } & \multicolumn{2}{c|}{ Anthropometric } & \multicolumn{2}{c|}{ Combined } \\
\hline & CA & BA & CA & BA & CA & BA \\
\hline MLR & 74.24 & 32.75 & 1.07 & 39.08 & 18.14 & 46.11 \\
\hline KD & 22.68 & 157.59 & 4.77 & 1677.2 & 13.35 & 1006.12 \\
\hline DNN & 74.25 & 439.83 & 132.85 & 56.76 & 228.84 & 180.77 \\
\hline Centroid1 & 81.51 & 689.37 & 86.45 & 1103.5 & 83.68 & 744.16 \\
\hline Centroid2 & 91.23 & 767.75 & 68.01 & 1137.21 & 65.00 & 777.02 \\
\hline Medoid1 & 115.75 & 704.42 & 87.85 & 986.13 & 107.62 & 745.16 \\
\hline Medoid2 & 152.22 & 712.11 & 69.50 & 1047.91 & 89.98 & 771.75 \\
\hline
\end{tabular}

Corresponding p-values are significant. Results are reported as $\chi^{2}$-distance. 


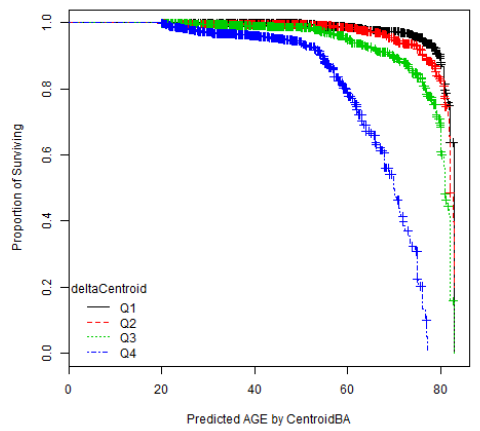

(a) biomarkers(using BA)

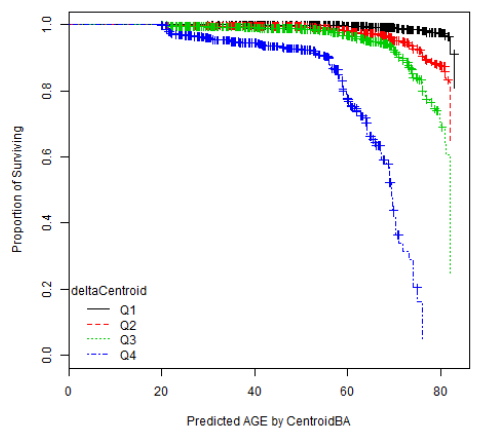

(c) anthropometric(using BA)

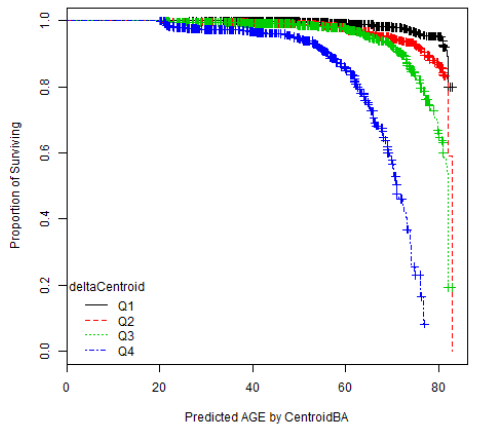

(e) combined(using BA)

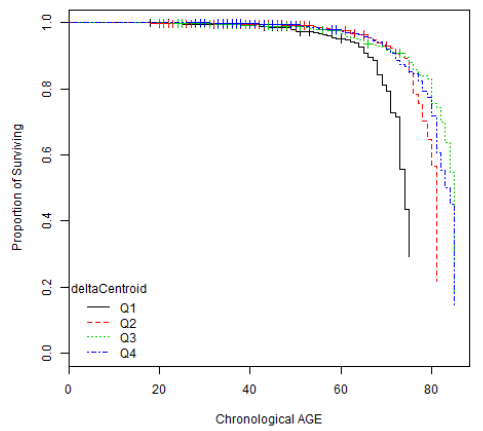

(b) biomarkers(using CA)

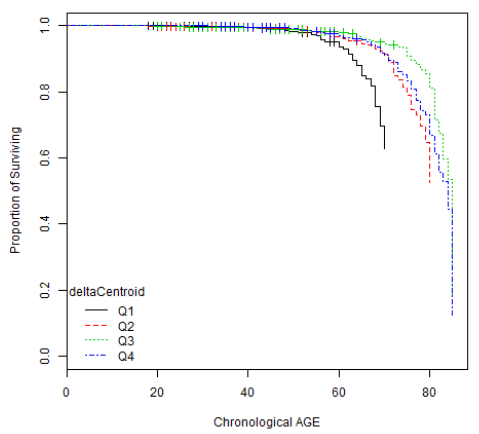

(d) anthropometric(using CA)

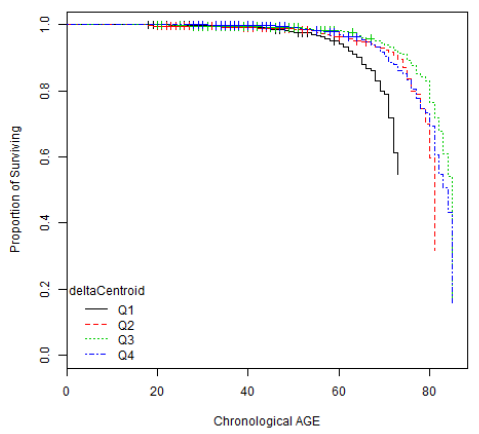

(f) combined(using CA)

Figure 4.9: The Kaplan Meier curves for using proposed centroid-based biological age (a, c, e), and chronological age (b, d, f). Results are reported for applying $\Delta=C A-B A$ on biomarkers, anthropometric features, and combined features. Q1, Q2, etc. denote 1st quartile, 2nd quartile, etc. 


\subsubsection{Human Anthropometry can predict BA}

Rahman and Adjeroh [118 showed relations between anthropometric measurements and chronological age. The next challenge is to investigate if body measurements can predict biological age. Some anthropometric features are correlated with biomarkers e.g., hemoglobin (HGB) has a correlation of 0.4 with height, ABSI has a correlation of 0.28 with systolic blood pressure and they both are correlated (ABSI $\rho=0.5$ and systolic blood pressure $\rho=0.55)$ with age, waist-to-height ratio (WHtR) has a correlation of -0.28 with albumin and WHtR is correlated with chronological age $(\rho=0.36)$. These observations suggest there might be a relation between anthropometric features and biological age. We apply the proposed algorithm on the same subjects in the dataset, but using anthropometric features only. Table 4.11 also shows the results for $\mathrm{Cox} \mathrm{PH}$ when applied on the estimated BA by anthropometric features. Proposed centroid BA has competitive performance with KD and MLR. We observe that for BA estimated BA MLR, delta applied to Cox model with chronological age is not reliable (p-value $=0.18)$. Fig. 4.9(c) and (d) shows KM curves for

estimated BA algorithms using anthropometric features. Performance of anthropometric features resembles the performance of biomarker features for both the chronological and biological age with combination of delta factored into quartiles. Table 4.12 shows results for KM curves using log-rank test. All the methods (except DNN) have lower $\chi^{2}$ distances using $\mathrm{CA}$ than the corresponding BA in the log-rank model.

\subsubsection{BA Estimation using Combined Features}

Fig 4.10 (a) shows how average height and average glycated hemoglobin vary with age. Glycated hemoglobin has correlation coefficient of 0.33 with age, and height has a correlation of -0.14 with age. But the correlation between height and glycated hemoglobin is -0.06 . Although they are not correlated with each other they are reasonably correlated with age. Similarly, Fig 4.10 (b) shows the variation of SBSI and pulse on an average with age. SBSI 

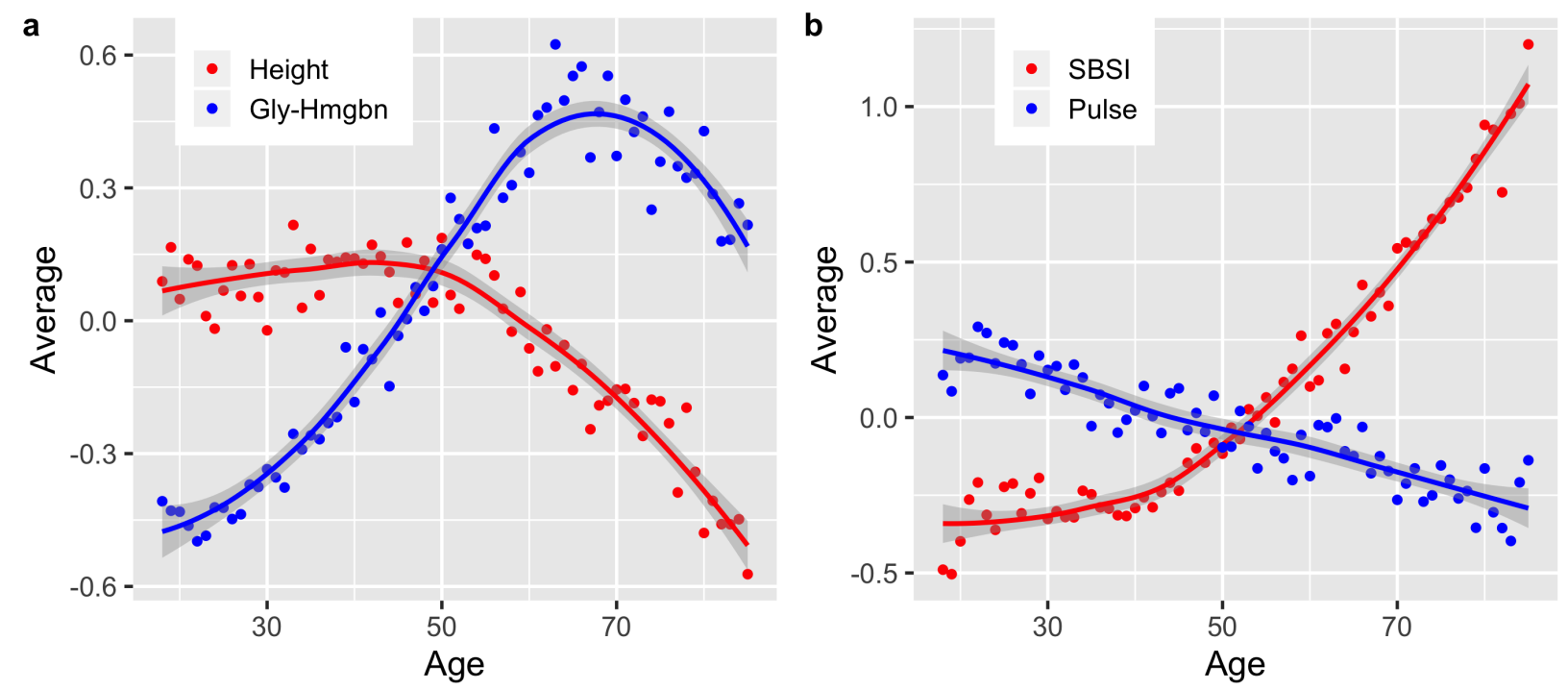

Figure 4.10: Pairwise uncorrelated attributes which are correlated with age.

has a corrleation of 0.39 with age, and pulse has a correlation of -0.14 with age, while SBSI and pulse are not very correlated $(\rho=0.07)$. Cevenini et al [33] proposed that biological aging rate differs for different set of attributes i.e., different tissue or cell can age differently. This is known as 'heterogeneous aging phenotype'. Body measurements and blood biomarkers are not highly correlated in many cases. Since these two types of features capture different aspects of human body, important information might be gleaned by the fusion of the anthropometric and biomarker features. We have combined both sets of features and applied the proposed BA estimation algorithms.

To place the results of our proposed approaches in perspective, we have compared with current popular approaches to BA estimation using biomarker data, namely KD [83], MLR [89], and DNN [114]. We have shown the corresponding results with these methods while we discuss the results of our proposed approaches (see Tables 4.11, 4.12). The results show that our proposed Approach using Centroid $\left(C_{\mu}, C_{\sigma}\right)$ have the best overall results.

In this work, we studied human biological age estimation using blood biomarkers, anthropometric measurements and the combined features. We presented generalized centroid and 
medoid of age neighborhood-based models to estimate biological age. The proposed method utilized selected age neighborhood to perform biological age estimation. Practical results demonstrate the significant improvement in BA estimation using the proposed methods when compared with existing approaches, such as KD [83], MLR [89], and DNN [114]. We established that centroid based method can be used on blood biomarkers, body measurements, or their combination to estimate biological age. 


\section{Chapter 5}

\section{Deep Learning for Analyzing Biological Age Using Physical Activity}

\section{$5.1 \quad$ Introduction}

A recent popular study [63] shows that more than $27.5 \%$ of adults had insufficient physical activity worldwide. The study included 358 population-based surveys in 168 countries with a total of 1.9 million participants 11]. Numerous health risks such as hypertension, diabetes, mental health, and weight-gain are related directly to physical activity [63]. With aging, intensity of physical activity decreases/reduces for older people [32,134, and this is more evident for females [42]. Various organizations [8,11] have recommended levels of physical activity for different age groups. However, the exact relationship between physical activity and aging is still unclear [115]. For instance, there is still the question of whether physical activity can predict age 43 .

The process of aging is complex and affects all biological systems. Age has a deep connection with health and mortality $21,89,97$. In general, a younger person is expected to have a better health condition, to be physically more active, and to have lower mortality hazard in comparison with a relatively older person. But two different people of the same chronolog- 
ical age may have very different health profiles and mortality hazards. This brings up an important classification of age, namely, chronological age versus biological age. Chronological age is based on the date of birth. However, biological age is a conceptual idea that a person's true age can be different from his/her chronological age. Although biological age is a loosely used concept and lacks precise definition, it is often viewed as the true age of an individual [83]. Thus, biological age provides a better measure of the life expectancy of an individual than his or her chronological age. The common idea is to calculate biological age based on some age-dependent variables [17, 35, 74, 127], where chronological age may or may not be a required variable depending on the application. In this work, our focus is on biological age. In particular, we investigate the question of whether human physical locomotor activity as recorded using a wearable device can be used for reliable estimation of biological age in adults.

Putin et al [114 studied the use of biomarkers in a deep learning framework for chronological age prediction. In this work they utilized an ensemble of multiple deep neural networks (DNNs) and trained on blood biochemistry samples. They employed a variation of the implementation of permutation feature importance (PFI) [16] technique to evaluate the relative importance of each blood biochemistry marker to ensemble accuracy. The best performance by a DNN was MAE of 6.07 years in predicting chronological age and the ensemble learning produced MAE of 5.55 years. They identfied the 5 most important biomarkers for predicting human chronological age: albumin, glucose, alkaline phosphatase, urea and erythrocytes. Fischer et al [49] earlier identified four biomarkers: alpha-1-acid glycoprotein, albumin, verylow-density lipoprotein particle size, and citrate for predicting all-cause mortality by applying biomarker profiling via nuclear magnetic resonance spectroscopy. They also showed healthy people may be at short-term risk of dying within 5 years from heart disease, cancer, and other illness. Findings from these studies suggest that particular biomarker can be related to aging and mortality (for example albumin). Cole et al [38] studied the use of structural neuro-imaging MRI under a Gaussian process regression framework. The predicted age was 
identified as 'brain-predicted age' or brain age for short. They combined DNA-methylation with brain age and showed that the combination improves mortality risk prediction. On the contrary, they also combined brain age with grey matter and cerebrospinal fluid volumes, but that did not improve mortality risk prediction. Pyrkov et al [115] applied convolutional neural network (CNN) on a week long physical activity data (NHANES 2003-2006) measured per minute. They applied a four-layer 1 dimensional CNN followed by two dense layers and a single unit layer to build the network.

\subsection{Methodology}

\subsubsection{Datasets}

Activity Dataset \& Preparation. We used physical activity data from the National Health and Human Nutrition Examination Surveys (NHANES) 2003 - 2004 and 2005 2006. NHANES uses a complex cluster design to sample members of the civilian USA population who are not institutionalized. Activity data is provided for a subset of NHANES participants. For these participants, NHANES provides physical (locomotor) activity for a 7-day continuous tracking of "activity counts" that is sampled every minute and recorded using a physical activity monitor (ActiGraph AM-7164 piezoelectric accelerometer (https: //www.actigraphcorp.com//). For this work, we analyzed the intensity of the physical activity, (also called device intensity value) recorded by the physical activity monitor. The devices were worn on the right hip by the individuals using an elastic belt. The combined dataset were of 14,631 study participants (7,176 in 2003-2004, and 7,455 in 2005-2006). Ethnicity included white, black, Mexican and others. Initially, we removed outlier samples with abnormally low (average activity count $<50$ ) or high $(>5000)$ physical activity count. We excluded days with less than 200 minutes corresponding to activity states $>0$, similar to Pyrkov et al [115]. Only participants with 4 or more days that passed this additional filter were retained, yielding a total of 7104 individuals with 586 deaths ( follow up in 2015). 
Anthropometric \& Biomarkers Dataset. We also used NHANES 2003-2006 anthropometric and biomarkers dataset. This dataset was used to study the potential relationship between human physical activity and the biomarkers. Numerous prior studies 21, 127] established connections between biomarkers and biological aging. Also some recent anthropometry based body shape indices such as surface based body shape index (SBSI) [118], and a new body shape index (ABSI) [85] showed better prediction of all cause mortality because of their relation with aging. Anthropometric measurements included BMI, height, weight, and waist circumference. The biomarker dataset included information on systolic and diastolic blood pressure, albumin, HDL, LDL, hemoglobin. After refining and merging the two datasets, we obtained 4268 individuals common to both datasets, aged 18 to 84 years with 329 deaths ( follow up in 2015).

\subsubsection{Data Transformation \& Data Representation}

Data Transformation. Given the nature of the time-series human locomotor data, with noise, and outliers, our first step is to apply some basic data transformation operations, such as smoothing and filtering. Given the data is a sequential time series data of 7 days, we tried moving averages on the data while varying the window sizes. The values of the physical activity intensity ranges over a large magnitude and moreover the intensity values are always positive. Thus we applied log transformation on the data (since there are '0' values in the original data, we added a negligible value (1) before applying log transformation). Another typical filtering operation on time series data is to apply moving averages that helps smoothing out the noise or outliers and also helps to find/extract long-term trends. We applied different types of moving averages (e.g., simple moving average (SMA), weighted moving average (WMA), and exponentially weighted moving average (EMA)). SMA is the unweighted mean of the previous $n$ data. Given we have physical activity of per minute $p a_{i}$, for an $n$-length window simple moving average would be $p a_{s m a}^{(i)}=\frac{1}{n} \sum_{j=0}^{n-1} p a_{i+j}$. EMA is a first order infinite impulse response filter that applies weighting factors which decrease 
exponentially. The EMA for physical activity can be calculated recursively: $p a_{e m a}^{(i)}=p a_{i}$, if $\mathrm{i}=1$ or $p a_{e m a}^{(i)}=\alpha * p a_{i}+(1-\alpha) * p a_{i-1}$, where the coefficient $\alpha$ denotes the weighting factor (ranges between 0 and 1). We have used $\alpha=\frac{2}{N+1}$. EMA has the best overall results. Moving averages were applied after the log transformation of the data. We also applied the Box-Cox transformation [25]. The Box-Cox transformation is a point transformation defined as $y_{\lambda}(x)=\left(x^{\lambda}-1\right) / \lambda$, if $\lambda \neq 0$ or $y_{\lambda}(x)=\log (x)$ if $\lambda=0$.

Data Representations. Pyrkov et al [115] applied the one dimensional data (10080 minutes) to a deep learning model to predict the biological age of a person. The basic idea was to use a one dimensional CNN architecture/model to extract features. This however, does not account for the temporal information in the data or the temporal pattern that a person might exhibit. For an improved representation of the potential temporal signals of biological age in the locomotor activity data, we consider the data as a temporal sequence of daily activity records. Each record is considered as a two dimensional matrix of size $24 \times 60$ capturing the locomotor activity for every hour and for each minute in the hour. The result is thus a three dimensional view of the locomotor activity data. This representation makes it easier to identify repeated temporal patterns in the data, which might provide cues to the functional or health status of the individual and thus to their biological age.

\subsubsection{LSTM estimation of Biological Age}

Given that we have a time series data with physical activity of a person in every minute for a week, it is natural to consider the use of a recurrent neural network to keep track of the time series data, in terms of memories of what the system has observed thus far. This is analogous to biological information/intelligence processing (i.e., a human processes information on incremental basis while keeping track of what he has processed so far). A recurrent neural network (RNN) has a 'state' that stores the information pertaining to what it has observed/processed thus far, and it processes sequential data through a number of iterations. So, an RNN is basically a neural network containing an internal loop and the state 
of the RNN is changed/reset between two sequences. The RNN, however, suffers from the problem of propagating vanishing gradients [69]. The Long Short-Term Memory (LSTM) is one of the most popular recurrent neural networks developed by Hochreiter and Schmidhuber [69] that adds a way to carry information across sequences. This saves information for later, and prevents older signals from vanishing gradually. Fig 5.1 (a) shows a basic LSTM cell. In the figure, $i_{t}$ is the input gate, $f_{t}$ is the forget gate, $c_{t-1}$ is the previous cell output, $o_{t}$ is the ouput gate, and $h_{t}$ is the final state. LSTM updates for timestep $t$ given input $x_{t}$, and the previous state $h_{t-1}$, and previous cell output $c_{t-1}$. The LSTM updates are given as follows 69:

$i_{t}=\sigma\left(W_{x i} x_{t}+W_{h i} h_{t-1}+b_{i}\right)$

$f_{t}=\sigma\left(W_{x f} x_{t}+W_{f} h_{t-1}+b_{f}\right)$

$c_{t}=f_{t} * c_{t-1}+i_{t} * \tanh \left(W_{c} x_{t}+W_{h c} h_{t-1}+b_{c}\right)$

$o_{t}=\sigma\left(W_{x o} x_{t}+W_{h o} h_{t-1}+b_{o}\right)$

$h_{t}=o_{t} * \tanh \left(c_{t}\right)$,

where $W_{x i}$ is the weight matrix for input gate $i$ and input $x$, and $b_{i}$ is the bias for $i$. Whenever a new input comes, the first step is to decide what information is going to be discarded from the cell state. This is performed by the forget gate. Then the next step is to get what information will be in the cell state. The $c_{t}$ first uses a sigmoid layer to decide which value to update and then a tanh layer provides a vector of new candiate values. Finally, the output will be based on the cell state with a filter (where a sigmoid decides the part of the output of cell state, and then finally the cell state is put through another tanh layer (output between -1 and 1) and multiplied by the output of the sigmoid gate).

Our basic approach to analyzing the human activity data is based on the idea of LSTM. We build on the concept of finding local patterns by applying the convolution operation of an image. Two major characteristics of convolutional neural networks (CNN) is that 


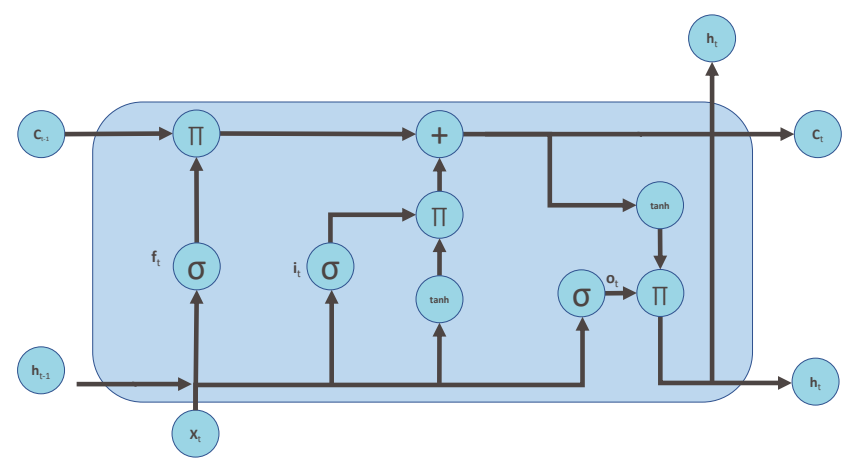

(a) LSTM

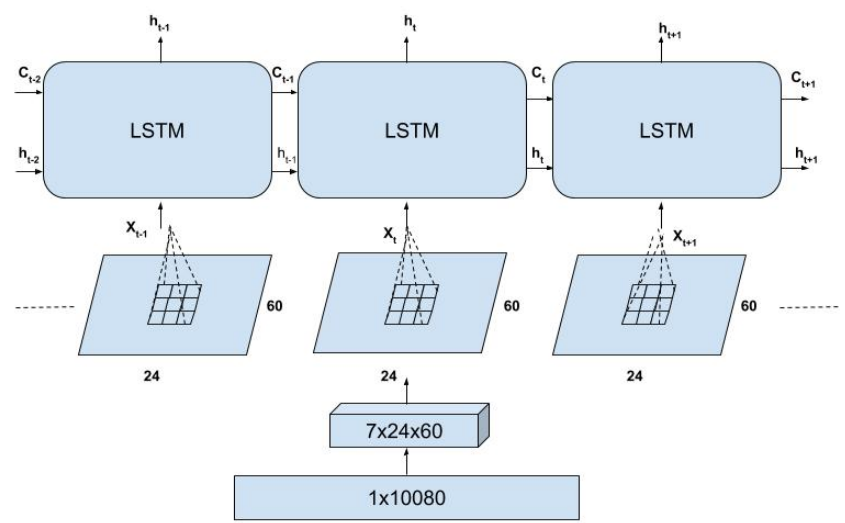

(b) Structure ConvLSTM*

Figure 5.1: (a) Basic LSTM cell. $\prod$ denotes multiplication, while + denotes addition, $\sigma$ is sigmoid function, tanh calculates hyperbolic tangent, (b) Transformation and inner structure of ConvLSTM* $^{*}$.

they can learn patterns in a hierarchical manner and the patterns learned are translation invariant (i.e., a learned pattern will be recognized anywhere). Our proposed method for estimating biological age is to apply the combination of LSTM with CNN to the human physical locomotor activity data. Note that this approach is different from the regular LSTM or CNN problem. Rather we take advantage of the structure in the sequence of $2 \mathrm{D}$ representations of the daily activities to learn valuable patterns from the activity data (which is very difficult using 1D CNN, LSTM, or DNN). We consider the data as a temporal 7(D) day information. Where each day has 24 hours and an hour is 60 minutes. So to break it down, we represent it as a two dimensional information of 24x60 (HxM) minutes with a temporal information of 7 days. The three dimensional information is therefore, 7x24x60 (DxHxM) minutes of data. Fig.5.1 (b) shows the representation described above. The image representation $(\mathrm{HxM})$ of the $1 \mathrm{D}$ physical data for each day introduce different feature dimensions that cannot be learned easily using 1D CNN architecture. In particular, using the $24 \times 60$ matrix representation of physical activity, records at minute 1 and minute 61 are neighbors (when considered as an image or matrix form), while in a one dimensional sequential view they are 60 timesteps apart. Two important factors here are that the spatial 
structure is changed, and the sequence of two dimensional information is very different from sequence of one dimensional information (especially, the information gathered from the 1D CNN and 2D CNN).

Our approach to combining LSTM and CNN builds on the idea of ConvLSTM proposed by Shi et al 144. Under ConvLSTM, the convolution structures are applied at both the input-to-state transition and at the state-to-state transitions. The ConvLSTM differs from simple CNN+LSTM in that, for CNN+LSTM, the convolution structure (CNN) is applied as the first layer and sequentially LSTM layer is applied in the second layer. Our approach also differs from ConvLSTM in that, we do not attempt to predict the next 2D matrix of physical activity for an individual, rather our goal is to estimate the biological age. Thus we concatenate two more fully connected dense layers and finally a single unit of neuron without activation to build up a scalar regression that estimates the biological age. We call our approach ConvLSTM*. Fig 5.2 shows the architecture of the proposed ConvLSTM* model. We also apply 1D CNN [115] and 1D DNN (see supplementary materials) on the 1D time series dataset to predict biological age. We compare the results from these three deep neural network models.

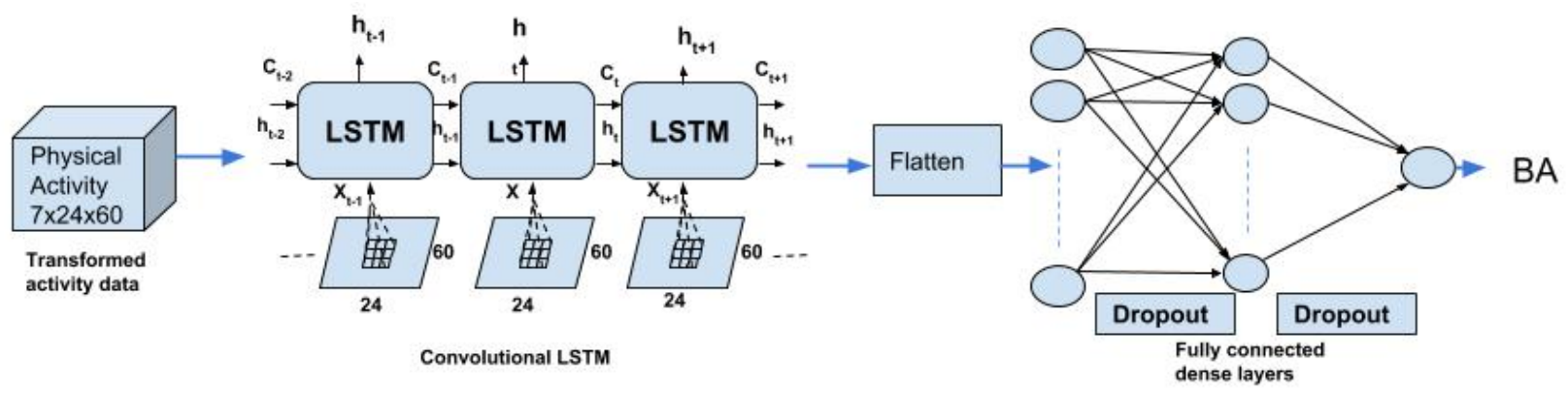

Figure 5.2: Architecture of proposed ConvLSTM* deep learning method for biological age estimation using human locomotor activity data. 


\begin{tabular}{|l|l|l|l|}
\hline & All (N=4268) & Female (N=2094) & Male (N=2174) \\
\hline Attributes & Average \pm SD & Average \pm SD & Average \pm SD \\
\hline C-reactive protein (mg/dL) & $0.37 \pm 0.82$ & $0.41 \pm 0.7$ & $0.33 \pm 0.92$ \\
\hline Glycated hemoglobin (Serum Albumin (ug/mL) & $4.23 \pm 0.4$ & $4.1 \pm 0.42$ & $4.36 \pm 0.33$ \\
\hline Total Cholesterol (mg/dL) & $197.96 \pm 42.56$ & $202.65 \pm 43.76$ & $193.44 \pm 40.88$ \\
\hline Serum Urea Nitrogen (mg/dL) & $12.34 \pm 5.49$ & $11.2 \pm 5.22$ & $13.43 \pm 5.52$ \\
\hline Serum Alkaline Phosphatase (U/L) & $70.69 \pm 27.45$ & $69.09 \pm 28.48$ & $72.24 \pm 26.33$ \\
\hline Systolic blood pressure (mm Hg) & $123.68 \pm 20.49$ & $121.58 \pm 22.84$ & $125.71 \pm 17.7$ \\
\hline Diastolic blood pressure (mm Hg) & $68.33 \pm 13.76$ & $66.74 \pm 14.13$ & $69.86 \pm 13.22$ \\
\hline Pulse (60 sec) & $71.95 \pm 12.55$ & $74.89 \pm 12.49$ & $69.11 \pm 11.95$ \\
\hline High density lipoprotein (mg/dL) & $55.95 \pm 16.59$ & $62.42 \pm 16.99$ & $49.72 \pm 13.54$ \\
\hline Hemoglobin (g/dL) & $14.39 \pm 1.5$ & $13.51 \pm 1.22$ & $15.25 \pm 1.23$ \\
\hline Lymphocyte percent (White blood cell count (SI) & $7.31 \pm 2.87$ & $7.47 \pm 2.72$ & $7.15 \pm 3$ \\
\hline Hematocrit (Red blood cell count (SI) & $4.71 \pm 0.52$ & $4.43 \pm 0.41$ & $4.98 \pm 0.47$ \\
\hline Platelet count (Body mass index (BMI) $\left(\mathrm{kg} / \mathrm{m}^{2}\right)$ & $26.44 \pm 4.61$ & $26.09 \pm 4.89$ & $26.77 \pm 4.3$ \\
\hline Surface based body shape index (SBSI) & $0.12 \pm 0.01$ & $0.12 \pm 0.01$ & $0.12 \pm 0.01$ \\
\hline Waist-to-height ratio (WHtR) & $0.56 \pm 0.08$ & $0.56 \pm 0.08$ & $0.55 \pm 0.08$ \\
\hline Age (years) & $46.3 \pm 20.18$ & $44.91 \pm 20.25$ & $47.65 \pm 20.02$ \\
\hline Average physical activity & $156.78 \pm 119.08$ & $136.88 \pm 111.66$ & $175.94 \pm 122.84$ \\
\hline
\end{tabular}

Table 5.1: Key attributes for study participants using the NHANES (2003-2006) dataset.

\subsubsection{Statistical Analysis}

We analyzed the data separately for male and female subjects, and for their combination. Table 5.1 shows the key attributes used in this study. We have used these 16 biomarkers. Table 5.1 also includes some important anthropometric measurements such body mass index (BMI), surface-based body shape index (SBSI), waist-to-height ratio, and average physical activity. SBSI was computed following Rahman and Adjeroh [118. We have used the following metrics to evaluate accuracy: (1) Pearson Correlation coefficient between $\mathrm{x}$ and $\mathrm{y}: \rho(x, y)=\frac{\sum_{i=1}^{N}\left(x_{i}-\bar{x}\right)\left(y_{i}-\bar{y}\right)}{\sqrt{\sum_{i=1}^{N}\left(x_{i}-\bar{x}\right)^{2}} \sqrt{\sum_{i=1}^{N}\left(y_{i}-\bar{y}\right)^{2}}}$, where $x$ and $y$ are different attributes, $N$ is number of samples. (2) Mean absolute error $M A E=\frac{1}{N} \sum_{i=1}^{N}\left|x_{i}-\hat{x}_{i}\right|$, where $x_{i}$ is the original value and $\hat{x}_{i}$ is the estimated value. In this work MAE shows the average change/error between the chronological age and the estimated age. (3) R-squared distance also known as coefficient of determination, $R^{2}=1-\frac{\sum_{i=1}^{N}\left(y_{i}-\hat{y_{i}}\right)^{2}}{\sum_{i=1}^{N}\left(y_{i}-\bar{y}\right)^{2}}$, where $y_{i}$ is the original value, $\hat{y}_{i}$ is the estimated value and $\bar{y}$ is the mean of $y$. To evaluate the estimated biological ages, we have applied two statistical models for validation and comparisons of BA algorithms, namely, Cox proportional hazard model (Cox PH) and Kaplan-Meier (KM) curves. We calculated the 
hazard ratio and corresponding p-value for Cox $\mathrm{PH}$ model using function "coxph" from $\mathrm{R}$ package "survival". Log-rank test was performed to quantify the KM plots. To perform log-rank test, we used "survdiff" function and calculated the chi-square distance and the corresponding p-value. In all cases, for p-value smaller than 0.05 the result is considered significant. Table 5.2 shows the correlation coefficient between average physical activity and the biomarkers. These results are based on the individuals that have both biomarkers and physical activity data available. The sample mean of age was 46.30 years, mean body mass index (BMI) is $26.44 \mathrm{~kg} / \mathrm{m}^{2}$, and mean surface based body shape index (SBSI) of 0.12.

All statistical analyses were performed using the R Language, Ver. 3.3.5 (The R Foundation for Statistical Computing, Vienna, Austria). The following packages were used: survival, gtools, ggplot2, tidyverse, Tensorflow, keras, reticulate, e1071, randomForest, matrixStats, TTR.

\subsection{Results}

\subsubsection{Human Locomotor Activity is associated with chronological age}

To motivate our study of deep learning methods for biological age estimation using the locomotor physical activity data, we first investigated whether there is any discernible association between physical activity and chronological age (which is easier to access than biological age). On average, physical activity has a correlation of -0.19 (p-value $=0.00)$ with chronological age (see Table 5.2). To further investigate the relation between physical activity and chronological age, we have grouped subjects in the physical activity dataset based on their age ranging from 18 to 84 . For subjects at a given age, we compute their average physical activity. Fig. 5.3(a) shows the results. We also calculate the standard deviation (SD) of physical activity for each person. Similarly, we calculate average of SD of

physical activity at each given age group. Fig. 5.3(b) shows the average SD for each age in our dataset. Average physical activity goes up from 18 to 45. After that we observe a linear 
decline of average physical activity from 46 to 85 years. However, standard deviations do not vary significantly from 18 to 45 although shows a slightly downward trend. Then it goes down linearly till age 85 . We observe a pattern of reducing physical activity on average with age. Standard deviation of physical activity on average also reduces after 45 years. There seems to be a cutoff around age 45 years, after that both average physical activity and SD of physical activity reduces linearly with age.



(a) Average physical activity

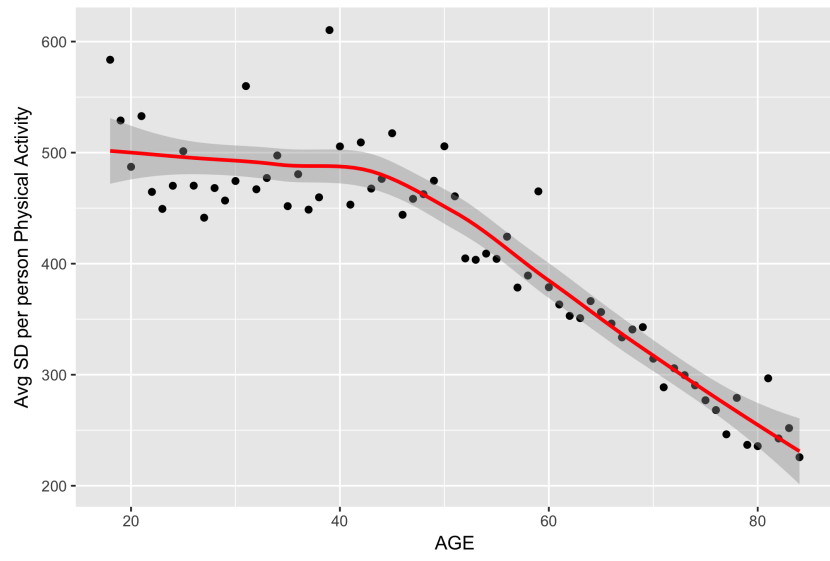

(b) Standard deviation average

Figure 5.3: Variation of average physical activity with age. Plotted for individuals grouped by year of age.

\subsubsection{ConvLSTM* estimated BA acceleration using physical activity data is associated with BA from biomarkers}

Biomarkers have been used frequently to estimate biological age in prior works [21, 127]. We refined and merged the physical activity dataset with biomarkers dataset, and obtained 4268 common individuals.

Association between Locomotor Activity with Biomarkers. To further investigate the potential effectiveness of aging from the locomotor activity data for predicting biological age, we analyzed the correlation of known biomarkers of aging with the average physical activity. For biomarkers of aging, we considered 16 of the biomarkers available in NHANES namely, C-reactive protein, glycated hemoglobin, albumin, total cholesterol, urea nitrogen, 
alkaline phosphatase, systolic blood pressure, diastolic blood pressure, pulse, high density lipoprotein, hemoglobin, lymphocyte percent, while blood cell count, hematocrit, red blood cell count, platelet count. Subsets of these have used in earlier work as key biomarkers of biological age $21,89,127$. Table 5.2 shows the correlation between the average physical activity and the biomarkers used. The table shows the correlation using direct measurements for Pearson's $\rho$ and corresponding p-value of the correlation. Albumin has the best correlation $(\rho=0.17$, p-value $=0.00)$. Red blood cell count, and hemoglobin have correlation of $0.15(\mathrm{p}$-value $=0.00)$. Interestingly, high density lipoprotein (HDL) has a very low correlation $(\rho=-0.024, \mathrm{p}$-value $=0.119)$ with physical activity. This result is similar to the study by Levine et al 89 where they showed HDL has a little correlation $(\rho=0.026)$ with chronological age. Fig. 5.4 shows how the two most correlated biomarkers (Albumin $(\rho=$ 0.17), and Hemoglobin $(\rho=0.15))$ vary with chronological age on average. From Fig. 5.3 and Fig. 5.4 we can observe that in general the average physical activity has a similar trend with the biomarkers (Albumin and Hemoglobin) with respect to age. In Figure 5.4 (a) average albumin goes down from ages 18 to 35 then goes up till about 45; from ages 45 to 84 albumin consistently goes down on average. Hemoglobin, however, goes up on average, from age 18 to 50, and then consistenly goes down till age 84. This is similar to the observation of Belsky et al 21] on the pace of aging. They showed that the pace of aging can be rapid for individuals who are biologically older than the peers i.e. a 38 year old with a biological age of 40 will age faster than a 38 year old with biological age of 38 years. Figure 5.3 also shows a similar property for average physical activity where the cutoff is around age 45 .

Similarity between estimated biological age from Biomarkers and estimated ConvLSTM* biological age from Locomotor Activity. Fig. 5.5(a) shows the estimated biological age using the Klemera and Doubal (KD) 83 method applied on the biomarker data and the corresponding estimated biological age using the proposed ConvLSTM* deep learning method applied on the locomotor activity data, for the 4268 individuals that have both physical activity and biomarker data available. Within the age range from 18 to 84 


\begin{tabular}{|l|c|c|c|c|}
\hline \multirow{2}{*}{} & \multicolumn{2}{|c|}{ Average Physical Activity } & \multicolumn{2}{c|}{ Chronological Age } \\
\cline { 2 - 5 } & Correlation & p-value & Correlation & p-value \\
\hline C-reactive protein & -0.083 & 0.000 & 0.048 & 0.002 \\
\hline Glycated hemoglobin & -0.086 & 0.000 & 0.340 & 0.000 \\
\hline Serum Albumin & 0.167 & 0.000 & -0.090 & 0.000 \\
\hline Total Cholesterol & -0.034 & 0.027 & 0.182 & 0.000 \\
\hline Serum Urea Nitrogen & -0.059 & 0.000 & 0.457 & 0.000 \\
\hline Serum Alkaline Phosphatase & -0.068 & 0.000 & 0.062 & 0.000 \\
\hline Systolic blood pressure & -0.110 & 0.000 & 0.535 & 0.000 \\
\hline Diastolic blood pressure & 0.049 & 0.001 & 0.099 & 0.000 \\
\hline Pulse & -0.084 & 0.000 & -0.211 & 0.000 \\
\hline High density lipoprotein & -0.024 & 0.119 & -0.014 & 0.364 \\
\hline Hemoglobin & 0.150 & 0.000 & -0.024 & 0.113 \\
\hline Lymphocyte percent & 0.059 & 0.000 & -0.066 & 0.000 \\
\hline White blood cell count & -0.067 & 0.000 & -0.053 & 0.001 \\
\hline Hematocrit & 0.147 & 0.000 & -0.011 & 0.486 \\
\hline Red blood cell count & 0.151 & 0.000 & -0.133 & 0.000 \\
\hline Platelet count & -0.038 & 0.013 & -0.116 & 0.000 \\
\hline Chronological age & -0.193 & 0.000 & & \\
\hline
\end{tabular}

Table 5.2: Correlation between average physical activity, chronological age and the biomarkers.

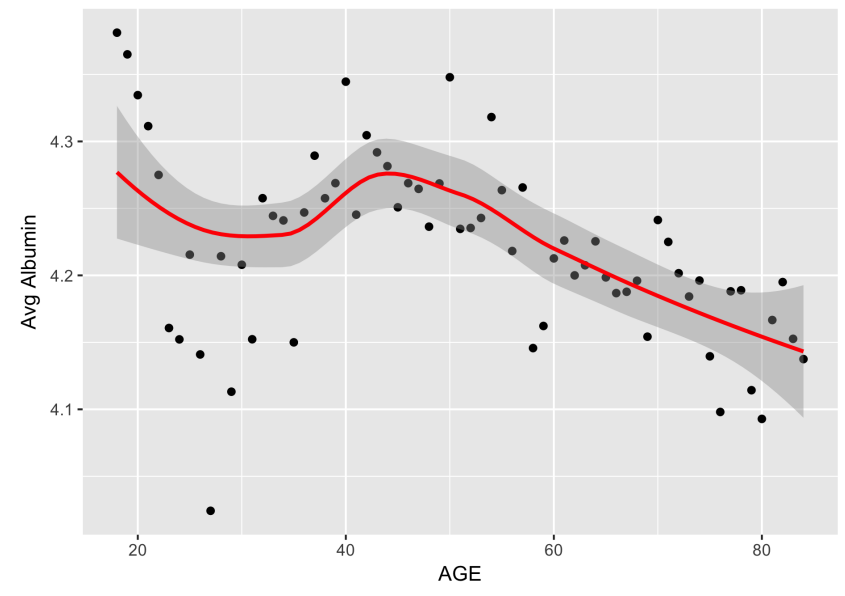

(a) Albumin

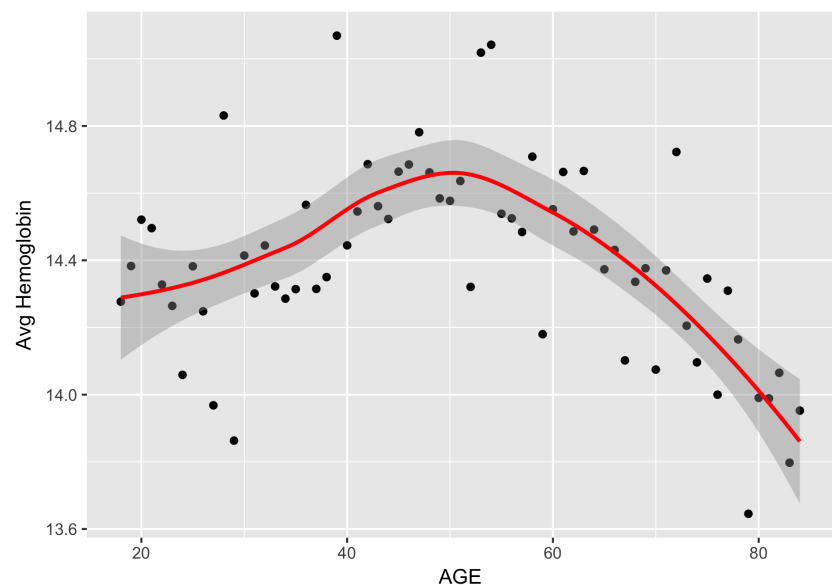

(b) Hemoglobin

Figure 5.4: Variation of two biomarkers (albumin, and hemoglobin) with age. Features plotted in average measurements for individuals grouped by year of age.

we took the individuals for each given age and calculated the mean of the estimated age. Both KD and ConvLSTM have similar results, with each producing BA estimates that increase consistently (generally linearly) with chronological age. At around age 47 years, we 
observe a crossover of the estimated BAs. This is pertinent with both the linear decrease of average physical activity in Fig. 5.3 and linear decrease of albumin and hemoglobin in Fig. 5.4 after age 45 years. Thus, it might be possible to estimate biological age based on physical activity of an adult individual. Fig. 5.5(b) shows the biological age acceleration using the estimated BA from both KD method on biomarker data and the proposed deep learning ConvLSTM* method on the locomotor activity data. Following Mitnitski et al [97] biological age acceleration is defined simply as $\Delta=C A-B A$. However, here we introduce the normalized form which we call normalized biological age acceleration (NBAA), denoted $\eta=\frac{\Delta}{C A}=\frac{C A-B A}{C A}$. This normalization is performed to reduce the effect of of large $\Delta \mathrm{s}$. For example, a $\Delta$ value of 5 could have different biological or health implications for an 18 year old $(\approx 28 \%$ difference) and for a 70 year old ( $\approx 7 \%$ difference). Our observation is that, overall, using $\eta$ values provided more improvements in the results when compared to using $\Delta$ values.

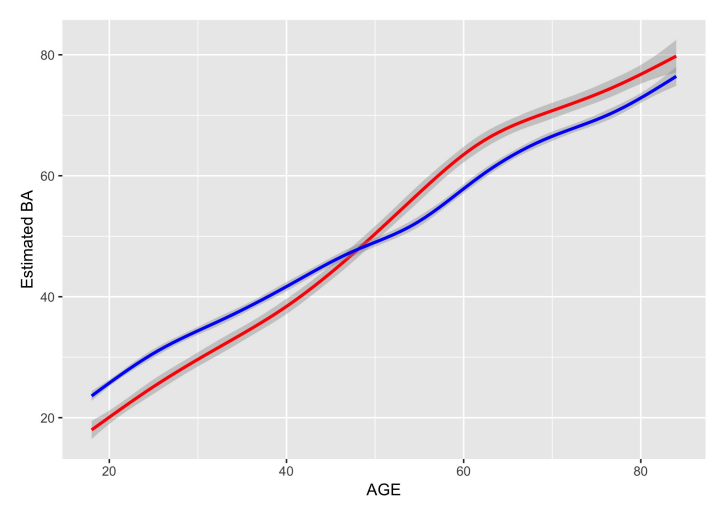

(a) Biological Age

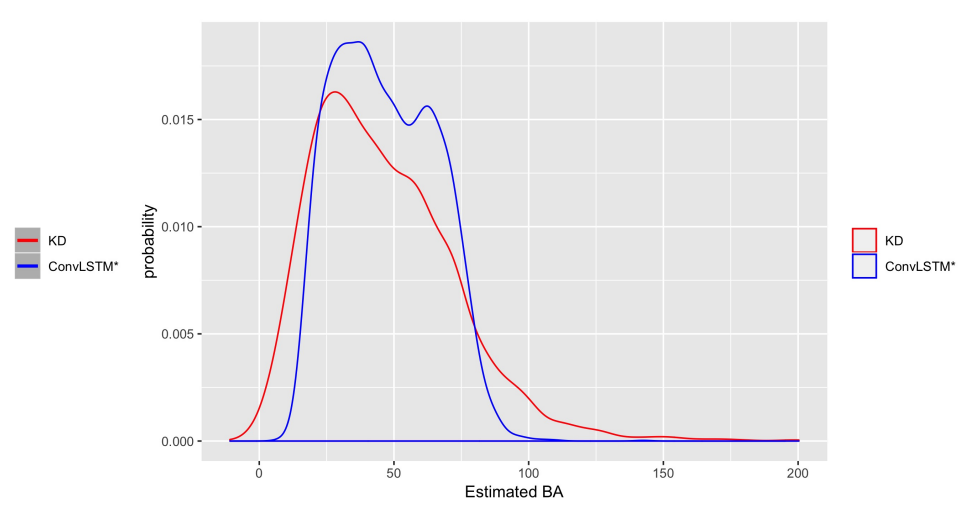

(b) density of estimated BA

Figure 5.5: Similarity between average biological estimated age using biomarkers using KD method and physical activity using ConvLSTM* over the age range 18-84. (a) Estimated biological ages, (b) density of estimated biological ages (These results are for common individuals in both the biomarker and physical activity datasets). 


\subsubsection{Deep learning biological age using ConvLSTM* on physical activity data leads to improved modeling of all-cause mortality.}

Biological age is a quantitative measure based on biological information of a person. Numerous approaches have used the idea of the association of physiological variables (biomarkers, activity) for estimating BA $21,38,70,115]$. However, given a new data modality, such as the human locomotor activity data studied in this work, a different model may be needed. Thus, we built a learning model using deep convolutional long short term memory (ConvLSTM) that is trained to estimate the BA. We applied two-dimensional ConvLSTM on the data that take advantage of both convolution and LSTM at the same time for extracting features rather than using the one-dimensional time series data. The proposed network architecture is shown in Fig. 5.1. To evaluate how well the estimated BA using the proposed deep learning ConvLSTM* approach on locomotor activity data captures the functional status of the subjects, we considered how the estimated BA relates to health risks. In particular, we studied the association of all-cause mortality with the normalized biological age acceleration $(\eta)$ using the estimated BA models.

\begin{tabular}{|c|c|c|c|c|}
\hline & \multicolumn{2}{|c|}{ Chronological Age } & \multicolumn{2}{|c|}{ Biological Age } \\
\hline & $\mathrm{HR}$ & p-value & $\mathrm{HR}$ & p-value \\
\hline 1D CNN 115 & 0.97 & 0.00 & 1.12 & 0 \\
\hline PCA 115 & 0.95 & 0.00 & 1.12 & 0 \\
\hline DNN & 0.78 & 0.00 & 1.06 & 0 \\
\hline ConvLSTM $^{*}(\lambda=1)$ & 0.98 & 0.02 & 1.14 & 0 \\
\hline ConvLSTM $^{*}(\lambda=0)$ & 0.97 & 0.01 & 1.13 & 0 \\
\hline ConvLSTM $^{*}(\lambda=0.9)$ & 0.97 & 0.00 & 1.13 & 0 \\
\hline
\end{tabular}

Table 5.3: Results of the Cox proportionality hazard (Cox PH) models applied on the normalized biological age acceleration $\eta=(C A-B A) / C A$ for estimated biological ages.

Cox PH model. We used Cox proportionality hazard modeling (Cox PH) 39, 84 and Kaplan-Meier (KM) curves [80] to quantify the association of the proposed ConvLSTM* estimated BA with all-cause mortality. Under the Cox model, the relationship between hazard and the covariates is described by considering the logarithm of the hazard as a linear function of the variables. Here we calculate the hazard ratio (HR) for each BA 
estimation algorithm. First we estimate biological age (BA) using 1D CNN [115, PCA 115, DNN, and ConvLSTM* models, and then we calculate $\eta=\frac{C A-B A}{C A}$ for each BA prediction algorithm. The DNN was developed and implemented in this work. The ConvLSTM* model is parameterized using the data transformation parameter, $\lambda$. Now we factorize $\eta$ (eta) to quantiles and use these to apply the Cox model. Table 5.3 shows the results for all the approaches. First, we applied chronological age in the Cox model. We observe that for 1D CNN, DNN, and PCA, the HR value is $0.97,0.78$, and 0.95 respectively. And the proposed ConvLSTM based method has similar results $\left(\operatorname{ConvLSTM}^{*}(\lambda=1), \mathrm{HR}=0.98\right.$, $\operatorname{ConvLSTM}^{*}(\lambda=0), \mathrm{HR}=0.97$, ConvLSTM$\left.{ }^{*}(\lambda=0.9), \mathrm{HR}=0.97\right)$. To further investigate, we use the estimated BA as the parameter for the Cox model rather than the age itself. Interestingly, hazard ratio (HR) is increased for all the methods. This is in line with prior work 21] that have shown that the estimated BA tend to be a better measure of health status compared with using the direct chronological age. From the perspective of Cox PH model, we found that proposed ConvLSTM* based BA prediction method has similar or slightly better performance than the other methods. Best overall results were obtained using ConvLSTM* $(\lambda=1)$, with $\mathrm{HR}=1.14$, p-value $\approx 0.000$ using $\eta$.

KM plots and LogRank. To further study the performance of the estimated BAs, we analysed the Kaplan-Meier (KM) survival curves obtained using the quantile factored eta $\left(\eta=\frac{C A-B A}{C A}\right)$. Fig. 5.6 shows the KM plots for the BA estimation algorithms. A given variable is a good mortality predictor if the Kaplan-Meier curves are easily distinguishable (more distance between them), and the variable gives a lower survival rates from low to high levels, with less crossing between curves. In general, each method of predicting biological age perform relatively well in distinguishing the proportion of survivors. From the figure, it is clear that when applied in survival model, using the estimated biological age performs better than using the chronological age. Among the deep learning BA estimation methods, the Pyrkov et al approach [115 using 1D CNN performed better than using the direct DNN model on the 1D data. However, once again, the proposed ConvLSTM* ap- 
proach (using $\lambda=1$ ) produced an improved result when compared with the Pyrkov et al's method [115]. To further quantify the performance, we used the log-rank test to compare the survival distributions obtained using different BA algorithms. The log-rank test compares the Kaplan-Meier curves to check if they are statistically equivalent. The output of the test is a $\chi^{2}$-distance, and the p-value associated with the distance. Higher $\chi^{2}$-distances and low p-values indicate a better separation between the curves, and hence a better performance in mortality modeling. The difference among the biological age estimation methods is more evident using quantitative measures, e.g., the $\chi^{2}$-distance between their respective KM curves, as captured by the log-rank test (Table 5.4).

\begin{tabular}{|l|c|c|c|c|}
\hline \multirow{2}{*}{} & \multicolumn{2}{|c|}{ AGE } & \multicolumn{2}{c|}{ PredictedAge } \\
\cline { 2 - 5 } & Chi-Sq & p-value & Chi-Sq & p-value \\
\hline 1D CNN & 20.98 & 0.00 & 141.57 & 0.00 \\
\hline DNN & 38.90 & 0.00 & 36.45 & 0.00 \\
\hline PCA & 13.00 & 0.00 & 80.72 & 0.00 \\
\hline ConvLSTM $^{*}(\lambda=1)$ & 4.71 & 0.19 & 123.56 & 0.00 \\
\hline ConvLSTM $^{*}(\lambda=0)$ & 8.95 & 0.03 & 142.09 & 0.00 \\
\hline ConvLSTM $^{*}(\lambda=0.9)$ & 14.78 & 0.00 & 132.59 & 0.00 \\
\hline
\end{tabular}

Table 5.4: Results of the log-rank test applied on the normalized biological age acceleration $\eta=$ $(C A-B A) / C A$ using the estimated biological ages.

\subsection{Discussion}

In this work we have investigated deep learning approaches on the NHANES (2003 - 2006) locomotor physical activity data. We estimate biological age (BA) based on the physical activity and chronological age (CA). To quantify how well the estimated biological age captures the health risk, we apply the Cox proportionality hazard model with all-cause mortality. The deep learning models such as DNN, CNN, ConvLSTM were trained to exploit the dependence of the physiological/activity changes with age. In all cases the deep learning approaches were trained to minimize the mean squared error (MSE) between estimated BA and CA, in every epoch. 


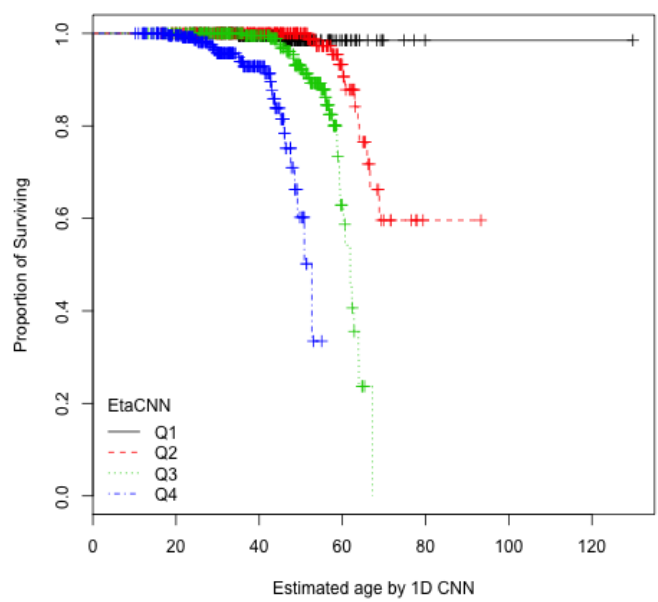

(a) $1 D C N N_{B A}$

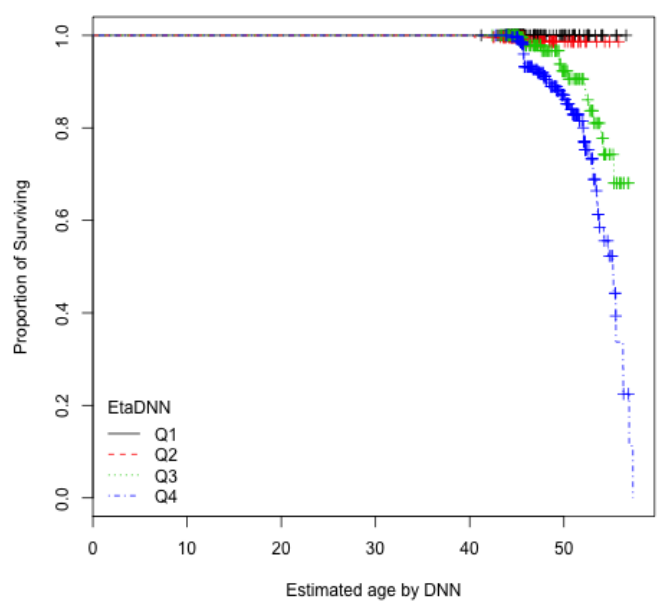

(c) $D N N_{B A}$

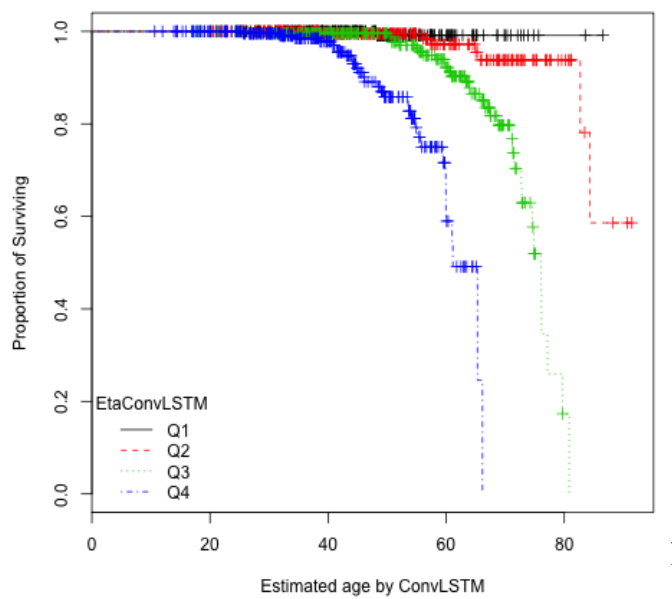

(e) ConvLSTM $M *_{B A}$

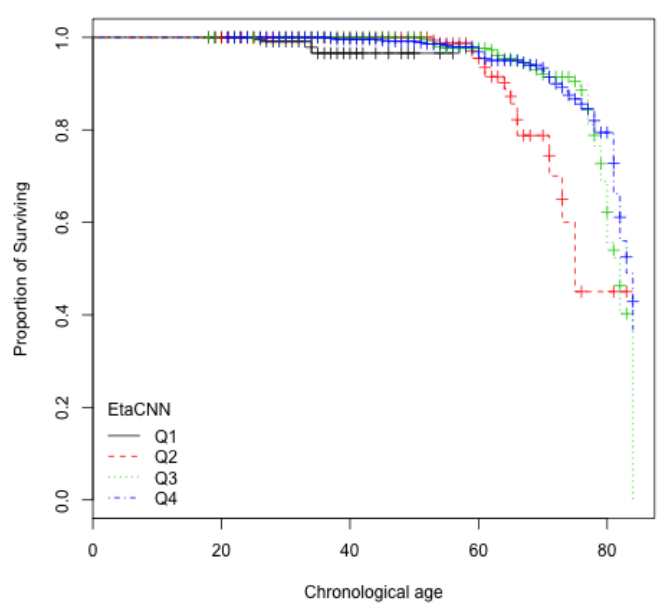

(b) $1 D C N N$

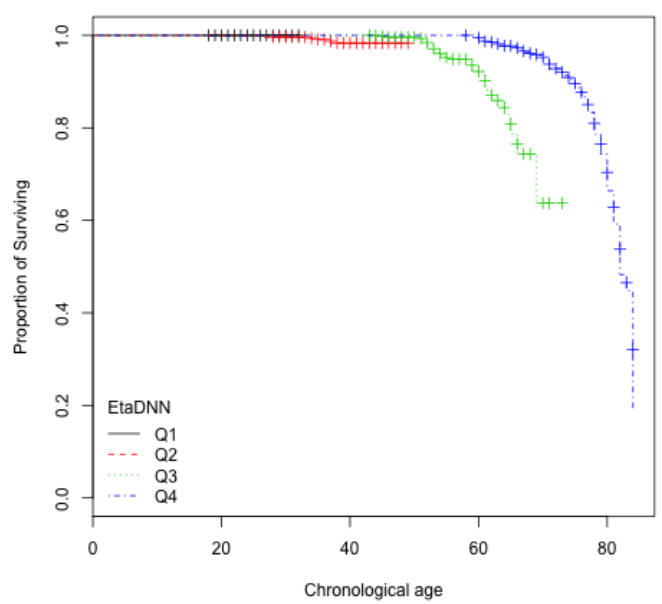

(d) $D N N$

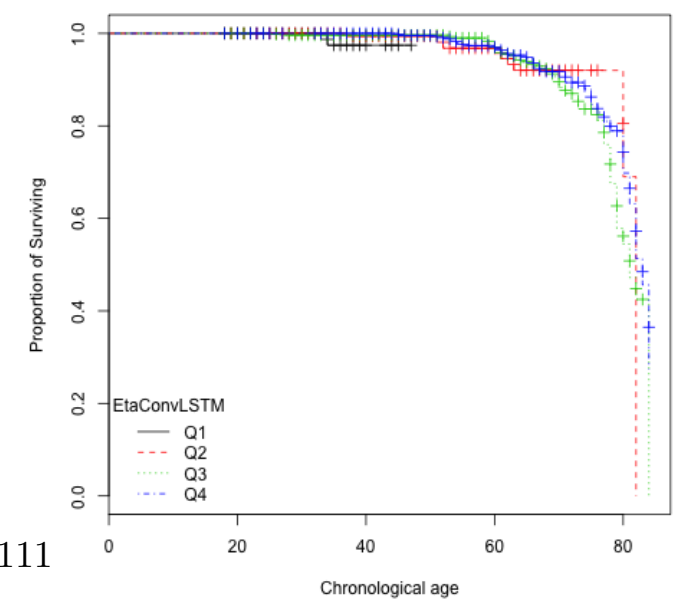

(f) ConvLSTM* 


\subsubsection{Parameter Choices}

We tested the performance of smoothening/filtering the original $1 \mathrm{D}$ activity data using different moving averages (simple moving average (SMA), weighted moving average (WMA), and exponential moving average ( EMA)). We observed that EMA provided the overall best result. To test the performance of window size $(\mathrm{N})$, we performed experiments using different values. We have considered $\mathrm{N}=1,2,4,8,10,16,20,25,30,35,40$. Table S1 shows the impact of the window size used for calculating the moving averages. We show the performance using different window sizes $(\mathrm{N})$ based on two machine learning algorithms, namely, support vector machine (SVM) and random forest (RF). Table S1 shows the variation of window size of exponential moving averages using the Box-Cox transformation. The performance criteria for choosing window size was to get lower MAE, higher R-squared distance, and higher correlation. From these results, we selected $\mathrm{N}=35$ as the best overall window size $\left(R^{2}=0.48\right.$ for SVM). Table 2 shows the impact of $\lambda$ for Box-Cox transformation. Best results were obtained with $\lambda=0.9\left(R^{2}=0.56\right.$ for SVM using $\left.\mathrm{N}=35\right)$. We have reported results for ConvLSTM* using three values of $\lambda ; \lambda=1$ (raw, with no transformation), $\lambda=0$ (log transformation), and $\lambda=0.9$.

For ConvLSTM* we have used 128 filters, a kernel size of $3 \times 3$ with a "ReLU" activation function. The first dense layer has 256 filters and second has 128 filters. Weight initialization was performed by Glorot and Bengio normal initialization [58], 30\% dropout [] was performed after each dense layer. We have tried different optimizer such as rmsprop [10], Adam [82], Nadam [45]. Based on the empirical results, we have selected adam optimizer for this work. Mean square error (mse) was used for loss function. We used Keras (https://keras. io/) library with Tensorflow (https://www.tensorflow.org/) in the backend to build the deep learning models. All experiments were performed using a NVIDIA 1080Ti graphics processing unit (GPU) running on a machine with Intel core-i7 processor and 32GB RAM. 


\subsubsection{Impact of Gender}

Results reported so far are from a single model that does not consider gender differences. That is, the same model is used for both female and male. However, gender is expected to have an influence on the performance of an age estimation scheme [54,62, 65]. Table 5.5 shows the results for separate gender specific models. We observe that for gender specific models applying normalized biological age acceleration $\eta=(C A-B A) / C A$ using estimated BA have higher hazard ratios than using chronological age. Moreover, for $\lambda=0$ and $\lambda=$ 1, using chronological age, the p-values are not significant. Using a separate model for male resulted in higher HR values (for each $\lambda$ ). However, using a separate model for female did not improve the hazard ratios than the single model for all.

\begin{tabular}{|l|c|c|c|c|c|c|c|c|}
\hline & \multicolumn{4}{|c|}{ Female } & \multicolumn{4}{c|}{ Male } \\
\cline { 2 - 9 } & Chronological Age & \multicolumn{1}{|c|}{ Biological Age } & \multicolumn{4}{c|}{ Chronological Age } & Biological Age \\
\cline { 2 - 9 } & HR & p-value & HR & p-value & HR & p-value & HR & p-value \\
\hline ConvLSTM $^{*}(\lambda=1)$ & 0.99 & 0.61 & 1.12 & 0.00 & 0.99 & 0.31 & 1.16 & 0.00 \\
\hline ConvLSTM $^{*}(\lambda=0)$ & 0.97 & 0.11 & 1.10 & 0.00 & 0.99 & 0.15 & 1.17 & 0.00 \\
\hline ConvLSTM $^{*}(\lambda=0.9)$ & 0.96 & 0.04 & 1.09 & 0.00 & 0.96 & 0.01 & 1.15 & 0.00 \\
\hline
\end{tabular}

Table 5.5: Results of the Cox Proportionality Hazard model (CoxPH) applied on the normalized biological age acceleration $\eta=(C A-B A) / C A$ for separate female, male subjects.

Fig. 5.7 shows the KM plots for $\eta=(C A-B A) / C A$ (ConvLSTM estimated BA) factored into quartiles. Separate male model's KM plots are of similar nature in comparison with the combined KM plots in Fig. 5.6(e). However, for separate female model the KM curve is slightly different although all the quartiles are well separated. To quantify these results more we perform log-rank test on the models. Table 5.6 shows the results for log-rank test for separate female and male models. Similar to the results of Cox PH models and KM curves, log-rank test also show better results for male model with higher $\chi^{2}$ values for all the $\lambda$ variations.

Relationship with Known Health Indices. Another way to investigate the performance of the proposed ConvLSTM* capturing health risks is to consider their possible relationship 


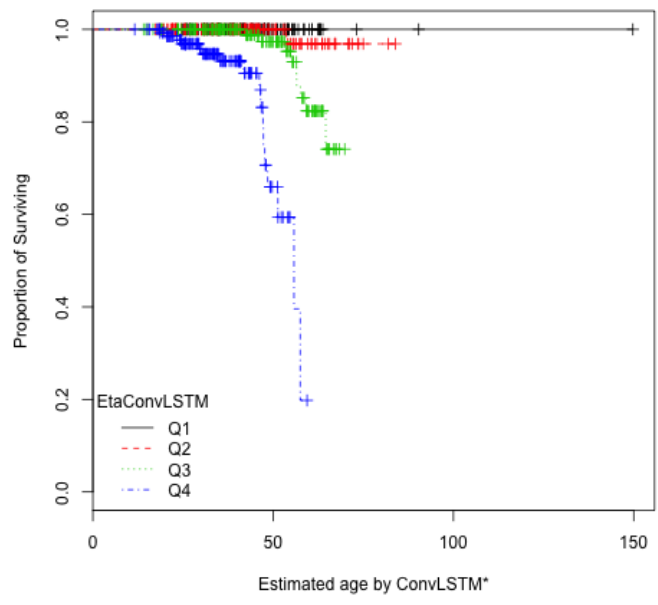

(a) female

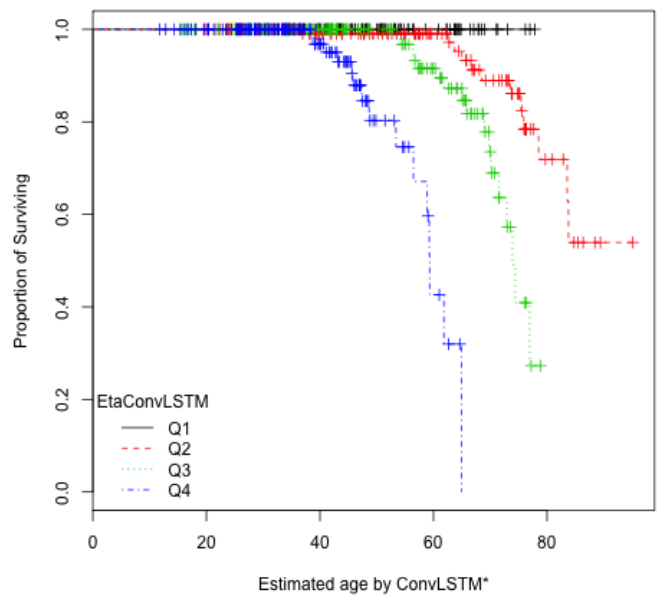

(b) male

Figure 5.7: The Kaplan Meier curves for applying $\eta=\frac{C A-B A}{C A}$ on the physical activity (a) female, and (b) male. Q1, Q2, Q3, and Q4 denote 1st, 2nd, 3rd, and 4th quartiles, respectively. The Eta prefix in the plot legend is followed by the method used to estimate BA and calculate $\eta$.

\begin{tabular}{|l|c|c|c|c|c|c|c|c|}
\hline & \multicolumn{4}{|c|}{ Female } & \multicolumn{4}{c|}{ Male } \\
\cline { 2 - 9 } & Chronological Age & \multicolumn{2}{|c|}{ Biological Age } & \multicolumn{2}{c|}{ Chronological Age } & \multicolumn{2}{c|}{ Biological Age } \\
\cline { 2 - 9 } & Chi-Sq & p-value & Chi-Sq & p-value & Chi-Sq & p-value & Chi-Sq & p-value \\
\hline ConvLSTM $^{*}(\lambda=1)$ & 1.98 & 0.58 & 82.28 & 0.00 & 1.37 & 0.71 & 99.89 & 0.00 \\
\hline ConvLSTM $^{*}(\lambda=0)$ & 1.64 & 0.65 & 30.01 & 0.00 & 4.59 & 0.20 & 143.05 & 0.00 \\
\hline ConvLSTM $^{*}(\lambda=0.9)$ & 6.86 & 0.08 & 54.96 & 0.00 & 19.86 & 0.00 & 92.96 & 0.00 \\
\hline
\end{tabular}

Table 5.6: Results of Log-rank tests applied on the normalized biological age acceleration $\eta=$ $(C A-B A) / C A$ for separate female, male subjects. 


\begin{tabular}{|c|c|c|c|c|c|c|c|c|c|}
\hline & \multirow[b]{2}{*}{ ConvLSTM* } & \multicolumn{2}{|c|}{$S B S I_{Q 1}$} & \multicolumn{2}{|c|}{$S B S I_{Q 2}$} & \multicolumn{2}{|c|}{$S B S I_{Q 3}$} & \multicolumn{2}{|c|}{$S B S I_{Q 4}$} \\
\hline & & Chi-sq & p-value & Chi-sq & p-value & Chi-sq & $\mathrm{p}$-value & Chi-sq & p-value \\
\hline \multirow{3}{*}{ All } & $(\lambda=1)$ & 26.73 & 0 & 14.25 & 0 & 77.72 & 0 & 177.13 & 0 \\
\hline & $(\lambda=0)$ & 28.61 & 0 & 25.25 & 0 & 100.24 & 0 & 287.38 & 0 \\
\hline & $(\lambda=0.9)$ & 44.84 & 0 & 49.85 & 0 & 109.83 & 0 & 290.13 & 0 \\
\hline \multirow{3}{*}{ Female } & $(\lambda=1)$ & 18.91 & 0 & 13.27 & 0 & 39.67 & 0 & 68.17 & 0 \\
\hline & $(\lambda=0)$ & 7.77 & 0.05 & 13.22 & 0 & 29.15 & 0 & 76.54 & 0 \\
\hline & $(\lambda=0.9)$ & 14.93 & 0 & 28.93 & 0 & 38.23 & 0 & 65.19 & 0 \\
\hline \multirow{3}{*}{ Male } & $(\lambda=1)$ & 11.94 & 0.01 & 5.64 & 0.13 & 57.95 & 0 & 143.36 & 0 \\
\hline & $(\lambda=0)$ & 7.58 & 0.06 & 8.21 & 0.04 & 78.62 & 0 & 194.05 & 0 \\
\hline & $(\lambda=0.9)$ & 16.07 & 0 & 12.1 & 0.01 & 43.86 & 0 & 139.56 & 0 \\
\hline
\end{tabular}

Table 5.7: Log rank results applying $\left(\eta=\frac{C A-B A}{C A}\right)$ for different SBSI categories. Results are shown for model with all subjects, female model and male models separately. Q1, Q2, etc. denote 1st quartile, 2nd quartile, etc.

with known indicators of health risk, such as the body mass index (BMI), waist to height ratio (WHtR), or the more recently introduced surface based body shape index (SBSI) 118 or ABSI [85]. In particular, we studied the variation of the proposed normalized biological age acceleration (NBAA, denoted $\eta$ ) computed using the estimated BA from ConvLSTM* with variations in the WHtR, and in SBSI categories. Earlier studies by Morkedal et al 99 have shown that the WHtR is a better measure of health status when compared with the BMI. Rahman and Adjeroh [118] made a similar observation on the superiority of SBSI over BMI. We have also observed the performance of ConvLSTM* with respect to the surface based body shape index (SBSI [118]) quartiles. Table 5.7 shows the log-rank test on the SBSI quartiles. The results are shown using $\eta$, for each SBSI category. We observe that, in general the $\chi^{2}$ values increase from first quartile to fourth quartile. However, the increase is not monotonic for all the variations of $\lambda$. For example, the $\chi^{2}$-distance decreased from $Q_{1}$ (26.73) to $Q_{1}$ (14.25) and then increased for $Q_{3}$ (77.72) and $Q_{4}$ (177.13) for $\lambda=1$ while for $\lambda=0.9, \chi^{2}$-distance increased monotonically from $Q_{1}(44.84)$ to $Q_{4}(290.13)$. We observe a similar trend for female-only and male-only models as well. The performance of ConvLSTM* with respect to the waist-to-height ratio (WHtR) quartiles is of similar nature to the results of BMI (4 group) and SBSI quartiles. Table 5.8 shows the log-rank test on the WHtR 
quartiles. We observe that, chi-square values increase from first quartile to third quartile. However, for the fourth quartile the chi-square values are not greater than third quartile although they are greater than both first and second quartile.

\begin{tabular}{|l|l|c|c|c|c|c|c|c|c|}
\hline & & \multicolumn{2}{|c|}{$W H t R_{Q 1}$} & \multicolumn{2}{c|}{$W H t R_{Q 2}$} & \multicolumn{2}{c|}{$W H t R_{Q 3}$} & \multicolumn{2}{c|}{$W H t R_{Q 4}$} \\
\cline { 2 - 10 } & ConvLTM $^{*}$ & Chi-sq & p-value & Chi-sq & p-value & Chi-sq & p-value & Chi-sq & p-value \\
\hline \hline \multirow{3}{*}{ ALL } & $(\lambda=1)$ & 11.79 & 0.01 & 63.97 & 0.00 & 135.45 & 0 & 102.48 & 0 \\
\cline { 2 - 10 } & $(\lambda=0)$ & 29.93 & 0.00 & 65.69 & 0.00 & 198.60 & 0 & 132.70 & 0 \\
\cline { 2 - 10 } & $(\lambda=0.9)$ & 27.50 & 0.00 & 111.42 & 0.00 & 222.06 & 0 & 196.86 & 0 \\
\hline \hline \multirow{3}{*}{ Female } & $(\lambda=1)$ & 17.33 & 0.00 & 20.41 & 0.00 & 76.16 & 0 & 35.02 & 0 \\
\cline { 2 - 10 } & $(\lambda=0)$ & 6.55 & 0.09 & 22.43 & 0.00 & 47.63 & 0 & 82.13 & 0 \\
\cline { 2 - 10 } & $(\lambda=0.9)$ & 7.17 & 0.07 & 26.87 & 0.00 & 53.97 & 0 & 77.86 & 0 \\
\hline \hline \multirow{3}{*}{ Male } & $(\lambda=1)$ & 4.60 & 0.20 & 46.17 & 0.00 & 100.66 & 0 & 81.80 & 0 \\
\cline { 2 - 10 } & $(\lambda=0)$ & 14.78 & 0.00 & 58.73 & 0.00 & 116.07 & 0 & 89.82 & 0 \\
\cline { 2 - 9 } & $(\lambda=0.9)$ & 4.26 & 0.24 & 38.26 & 0.00 & 108.31 & 0 & 93.79 & 0 \\
\hline
\end{tabular}

Table 5.8: Log rank results applying normalized biological age acceleration $\left(\eta=\frac{C A-B A}{C A}\right)$ for different WHtR quartiles. Results are shown for model with all subjects, female model and male models separately. Q1, Q2, etc. denote 1st quartile, 2nd quartile, etc.

\subsubsection{Comparison}

Pyrkov et al 115 proposed a deep learning architecture for analyzing the physical activity data that is based on a one dimensional convolutional neural network (CNN) architecture. We also implemented a deep neural network (DNN) to estimate biological age (Our own architecture and implementation; motivated from the architecture of Putin et al [114]). These models (DNN and 1D CNN) are used as comparative results. The results on mortality modeling using the Cox model and KM curves have shown the performance of the proposed ConvLSTM* in comparison with DNN and Pyrkov et al's 115 1D CNN. See Tables 5.3, 5.4 and Fig. 5.6. The results showed that the proposed ConvLSTM* method generally outperformed the 1D CNN or the DNN. Another way to compare the methods is by considering the estimated chronological age from the methods. Since the deep learning methods were trained to minimize the mean square error between the estimated and the original chronological age, we can compare the methods based on their performance in CA estimation. 


\begin{tabular}{|c|c|c|c|c|c|c|}
\hline \multirow{2}{*}{} & & \multirow{2}{*}{ 1D CNN 115 } & \multirow{2}{*}{ DNN } & \multicolumn{3}{|c|}{ ConvLSTM* $^{*}$} \\
\cline { 5 - 7 } & & & & $(\lambda=1)$ & $(\lambda=0)$ & $(\lambda=0.9)$ \\
\hline \multirow{3}{*}{ Test } & MAE & 17.3 & 17.1 & 12.6 & 13.21 & 13.4 \\
\cline { 2 - 7 } & RMSE & 22 & 23 & 15.74 & 16.81 & 16.74 \\
\cline { 2 - 7 } & CORR & 0.49 & 0.41 & 0.62 & 0.55 & 0.55 \\
\hline \hline \multirow{3}{*}{ Train } & MAE & 12.88 & 17.08 & 6.51 & 10.17 & 5.63 \\
\cline { 2 - 7 } & RMSE & 16.25 & 21.34 & 8.58 & 12.95 & 7.46 \\
\cline { 2 - 7 } & CORR & 0.52 & 0.79 & 0.92 & 0.79 & 0.94 \\
\hline \hline & epoch & 500 & 100 & 10 & 10 & 10 \\
\hline
\end{tabular}

Table 5.9: Results of the Deep learning Age Prediction methods.

Table 5.9 shows the mean absolute error (MAE), root mean square (RMSE), and correlation (CORR) for all the deep learning methods discussed. Results are reported for both training and test datasets. We observe that ConvLSTM* $(\lambda=1)$ on the original dataset has the lowest MAE (12.6), RMSE (15.74), and best correlation $(\rho=0.62)$. ConvLSTM* with $\lambda=$ 0 and $\lambda=0.9$ had similar performance $(\rho=0.55$ for both, and MAE of 13.21 and 13.4 respectively).

\section{Does improved CA estimation really imply reduced performance in BA estima-}

tion? All the methods described above use supervised learning that learns in the form of minimizing the difference between estimated biological age and the chronological age itself. This difference has been called biological age acceleration [97] in the literature. Pyrkov et al 115 suggested that an improvement in CA estimation can affect the BA acceleration significance for a particular test that may involve health risk. However, our results show that the proposed ConvLSTM* approach results in a better estimation for chronological age (lower MAE, higher correlation, and higher R-squared values) in comparison with the other methods. We have also shown that ConvLSTM* on the transformed data (using $\lambda=0$, 0.9) have better BA acceleration and better performance in modeling all-cause mortality using both the Cox PH model and KM curves than 1D CNN, DNN. We have also shown that, the resulting normalized biological age acceleration $(\eta)$ using the estimated BA from ConvLSTM* on the transformed activity values $(\lambda=0,0.9,1)$ resulted in a better overall 
performance in capturing health risks, for instance, in modeling all-cause mortality, when compared with the other deep learning methods, namely, 1D CNN [115 and DNN. These results seem to go against the assertion by Pyrkov et al 115 that improved CA estimation will typically lead to a deterioration in BA estimation. The issue might be on how the estimated BA is used for further analysis, rather than the accuracy of the initial chronological age estimation. This clearly warrants further investigation.

\subsubsection{Limitation}

We identify some limitations for this study. One potential problem is the lack of control for certain demographics, for instance, socio-economic status, ancestry, etc. The dataset was available for one week. Although this is a time series data and for each individual we have $10080(7 \times 24 \times 60)$ minutes of information, more data may reveal important information. The device (ActiGraph AM-7164 piezoelectric accelerometer) that is used to collect the data was worn on hip by each individual. Sometimes the device was removed from the body (e.g. during shower). We believe, a water resistant smart watch or a wristband type device would be easier to use from a user perspective and hence the activity records would be more accurate. 


\section{Chapter 6}

\section{Biological Age from 3D body shape}

\subsection{BA Modeling and Estimation using 3D Body Shape}

MakeHuman [20] is an opensource graphical user interface software that is designed specially for the prototyping of humans for 3D modeling. MakeHuman takes advantage of the 3D morphing technology. Starting from an average default human mesh, it can be transformed into a different variety of characters (male, female, Caucasian, Asian, adult, kid, etc.). We can generate different characters with very different body shapes.

Piccirilli et al 110,111] showed a virtual reality framework for the analysis of whole human body surface area. They generated a dataset based on virtual subjects. A new virtual environment to facilitate the analysis of human subjects in 3D was developed. They showed how the whole body surface area can be estimated using a single view image.

Building on this setup proposed by Piccirilli et al 110,111], we have built a plugin to generate from NHANES anthropometry measurements to 3D MakeHuman models. 


\subsection{Methodology}

\subsubsection{Dataset}

We identified 4268 individuals with information on their body measurements, and physical activity data generated from an accelerometer. Body measurements include weight, height, BMI, arm length, arm circumference, waist circumference, body surface area etc.

Fig. 6.1 shows example 3D meshes generated from sample subjects of NHANES for different BMI categories using separate models for male and female. The parameters that are used to generate these subjects are: gender, age, height, race, breast size, upper leg height, upper arm length, upper arm circumference, thigh-circumference, and waist-circumference.



(a) female

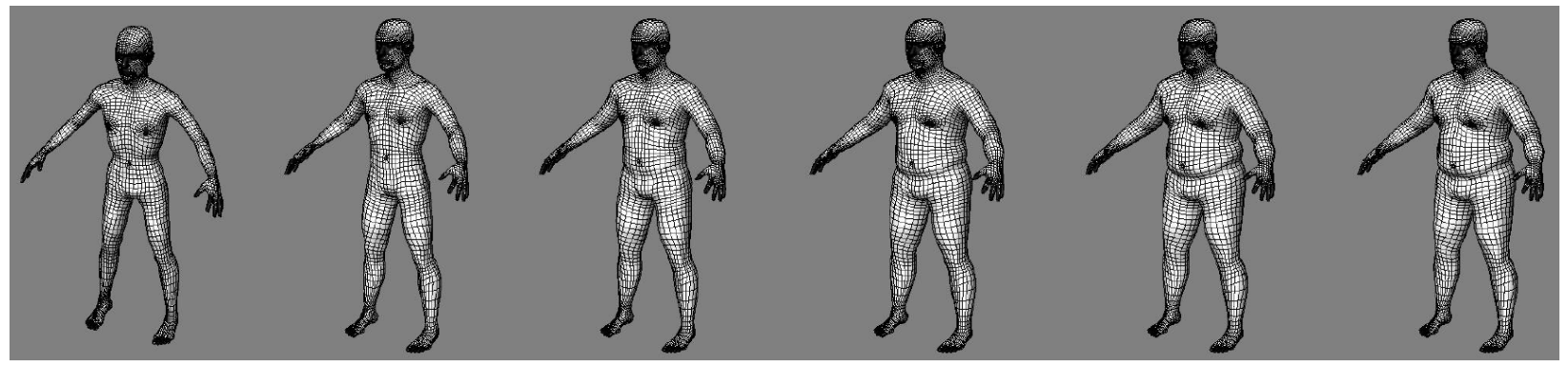

(b) male

Figure 6.1: Generated 3D meshes from sample subjects of NHANES for each BMI categories (a) female subjects Underweight (18.37), Normal (19.95), Overweight (29.73), Obese I (32.97), Obese II (38.57), Obese III (43.42) (from left to right respectively), (b) male subjects Underweight (16.96), Normal (23.65), Overweight (28.84), Obese I (32.49), Obese II (37.08), Obese III (45.34) (from left to right respectively). 


\subsubsection{NHANES to MakeHuman}

The converted 3D mesh dataset supposedly contain more sophisticated information about a person. In this chapter we investigate this dataset in more detail, and study the possibility of using the generated 3D body shape for biological age estimation. However, we understand that given the nature, this type of data can be very different than the original humans. It can be challenging because the plugin algorithm might generate a person based on the information that could be far from a real person.

We generated six 2D images for each person using MakeHuman, namely, front, back, left side, right side, top, and bottom views. These figures can be considered as the 3D representations of the person. Fig. 6.2 shows six figures for a female and Fig. 6.3 shows a male sample from the dataset.

In Chapter 5 we have shown that the proposed convolutional LSTM generally outperforms the one dimensional convolutional neural network (1D CNN) or the dense neural network (DNN). In this era of faster graphics processing units (GPU), more sophisticated deep learning models are being generated rapidly. In this chapter, we apply state-of-the-art deep learning techniques on the physical activity data and also on the 3D human generated using the MakeHuman from the NHANES dataset for human biological age estimation and quantification.

\section{Transfer Learning}

Transfer learning (TL) is a machine learning approach where a model learned from a task is re-purposed or reused on a different but related task [101]. The idea is to improve the learning for the second task based on the knowledge gathered from the first task. Transfer learning tends to work if both the tasks are general in principle. That is, the features are specific to the base task and unrelated to the second task, the transfer learning will probably not work well. Transfer learning can be used as a pre-trained model or as a develop model 


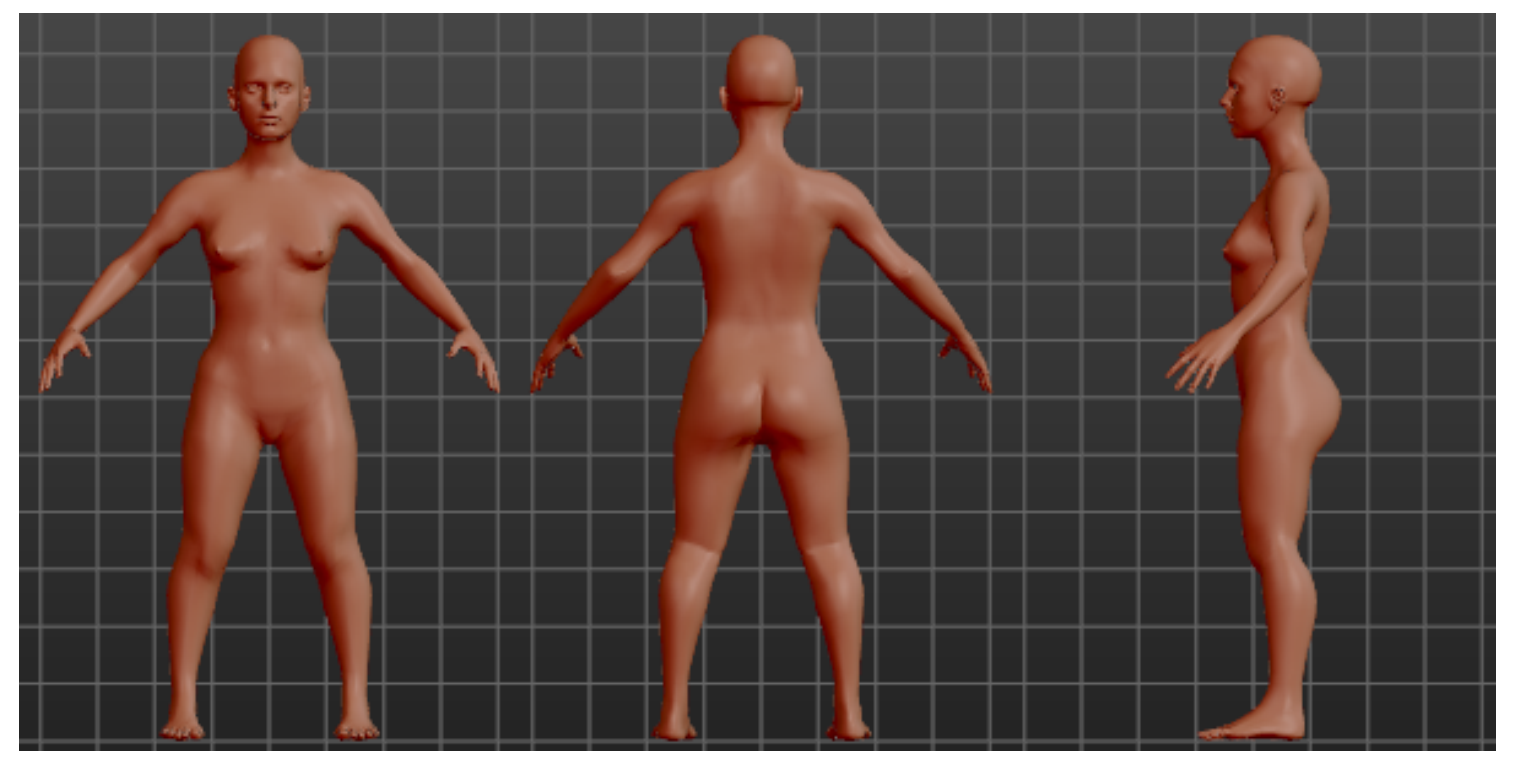

(a) front

(b) back

(c) left

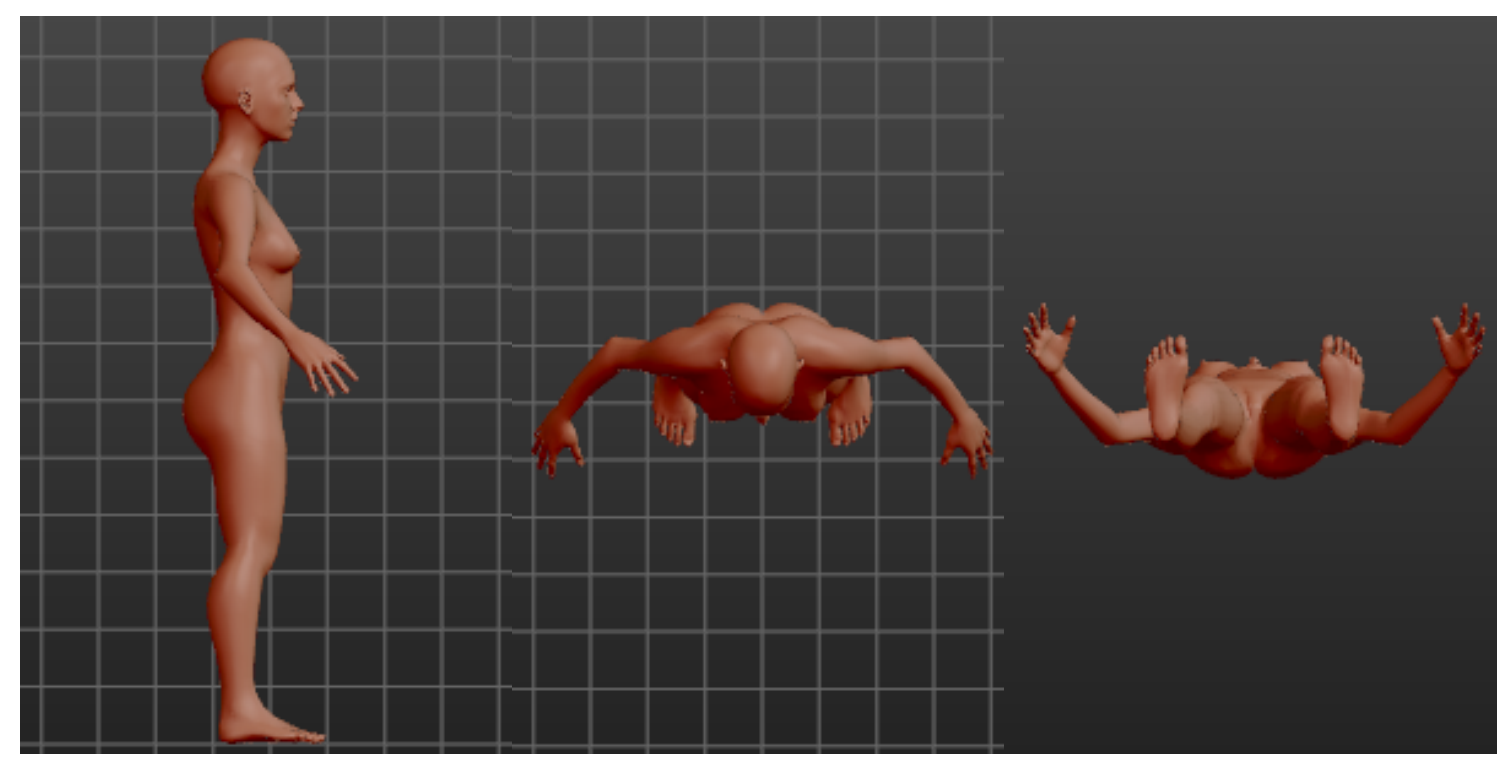

(d) right

(e) top

(f) bottom

Figure 6.2: Generated 2D images from NHANES from different viewing angles for a female subject (a)front, (b) back, (c) left, (d) right, (e) top, and (f) bottom. 


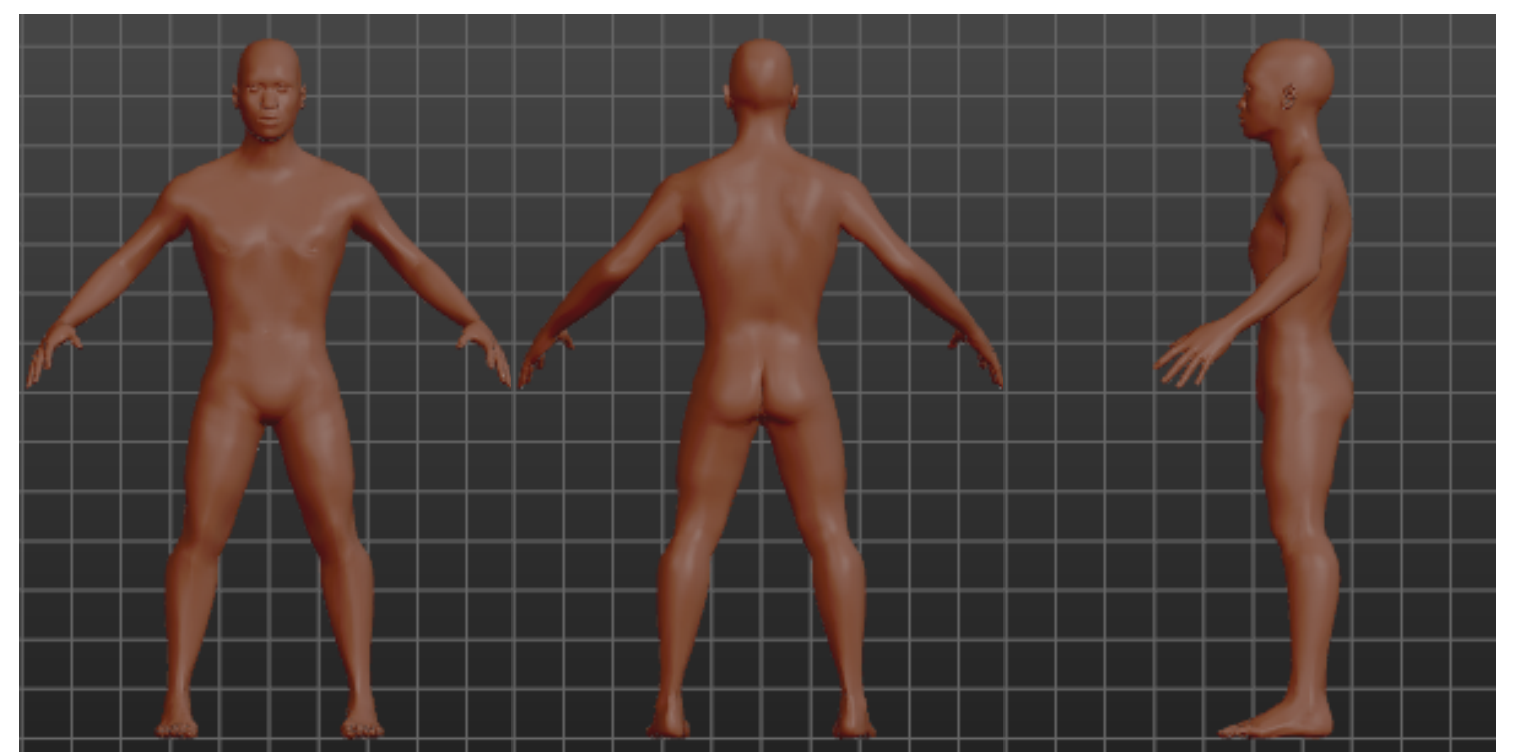

(a) front

(b) back

(c) left



(d) right

(e) top

(f) bottom

Figure 6.3: Generated 2D images from NHANES from different viewing angles for a male subject (a)front, (b) back, (c) left, (d) right, (e) top, and (f) bottom. 
approach. A number of pre-trained models on large and challenging datasets are now made available from different research institutes. We can select them from the pool for suitable cause. We can either reuse the model or tune the model for the specific task. However, when the pre-trained models are not available, we can develop our own custom model for the base task and later can be re-purposed for a model on the second task. We used transfer learning weights from ResNet-50 to compare the performance with the proposed approach.

\subsubsection{Multi-Input Deep Learning Models}

Using physical activities and anthropometric data separately performs reasonably for age estimation; fusing these multimodal information may improve performance of BA estimation. 3D body shapes generate by the MakeHuman present a different feature sets which can be combined with other modalities using fusion via deep learning. We study multi-input on feature and score level. The deep learning architecture that we tried in Chapter 5 are sequential models.

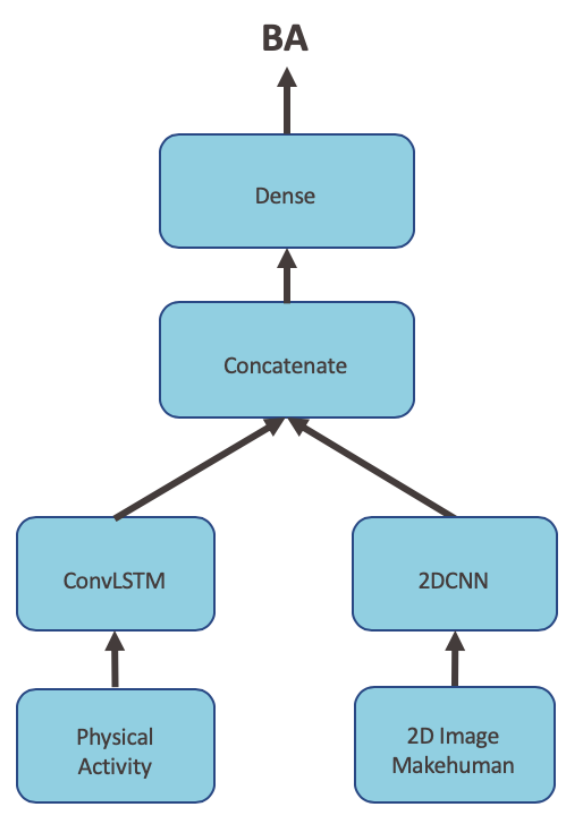

(a) model 1

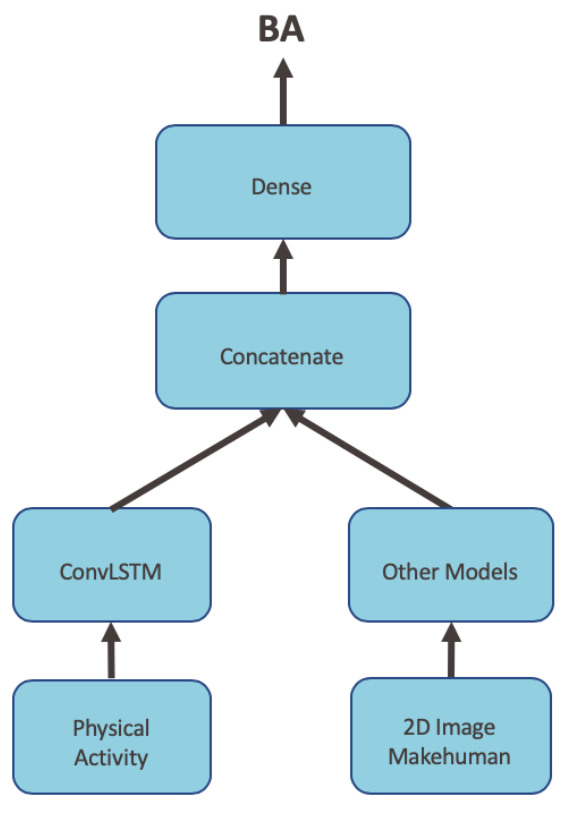

(b) model 2

Figure 6.4: Variations of multi-input model to estimate biological age. 
We apply multi-input models to glean more valuable patterns from different deep learning models. On the physical activity feature set, we apply convolution LSTM architecture as described in chapter 5. At the same time, we apply a 2D convolutional neural network on the MakeHuman generated images of the corresponding subjects. A naive approach would be to train two separate models and then to perform a weighted average of two models' performance. However, this may be suboptimal, since the information extracted by the models may be redundant. Rather we jointly learn a more accurate model of the data by using a model that can see all available input modalities simultaneously. We used transfer learning weights from ResNet-50 to compare the performance with our proposed approaches.

\subsection{Results}

In this chapter, for performance evaluation of biological age estimation algorithms we consider error in chronological age (CA) estimation and mortality modeling with survival analysis (Cox Proportional Hazard model, and Kaplan-Meier estimation).

\subsubsection{CA Performance}

Table 6.1: Results of the deep neural network age prediction methods.

\begin{tabular}{|l|c|c|c|}
\hline & MAE & RMSE & CORR \\
\hline Physical Activity (ConvLSTM) & 17.60 & 21.72 & 0.50 \\
\hline 2D Images (2D CNN) (front) & 39.99 & 44.72 & -0.05 \\
\hline 2D Images (2D CNN) (side) & 43.40 & 47.92 & -0.03 \\
\hline 2D Images (2D CNN) (back) & 42.31 & 46.92 & 0.01 \\
\hline 2D Images (2D CNN) (front, side, back) & 20.09 & 24.68 & 0.03 \\
\hline Combined Features (2D-CNN, ConvLSTM)(front) & 17.27 & 20.25 & 0.19 \\
\hline Combined Features (2D-CNN, ConvLSTM)(side) & 17.86 & 21.95 & 0.51 \\
\hline Combined Features (2D-CNN, ConvLSTM)(back) & 16.77 & 20.68 & $\mathbf{0 . 5 4}$ \\
\hline Combined Features (2D-CNN, ConvLSTM)(front, side, back) & $\mathbf{1 5 . 5 2}$ & $\mathbf{1 8 . 4 5}$ & 0.43 \\
\hline 2D Images (ResNet) (front, side, back) & 19.70 & 24.09 & 0.05 \\
\hline Combined Features (ResNet, ConvLSTM)(front, side, back) & 16.56 & 20.27 & 0.50 \\
\hline
\end{tabular}

Table 6.1 shows the mean absolute error (MAE), root mean square error (RMSE), and correlation (CORR) for the three deep neural networks discussed above. We observe that the combined features applied with two different models in parallel has the lowest MAE of 
17.27 (using front image only), while using three images (front, back, and side) gives the lowest MAE of 15.52 with a correlation of $0.43(\rho)$. However, combined features (ConvLSTM applied on the physical activity data and 2D CNN applied on the back image) provides the best correlation of 0.54. Only front 2D images used as a feature has a very high MAE of 39.99 with a correlation of -0.05 . The combination of three images using $2 \mathrm{D}-\mathrm{CNN}$ have a lower MAE of 17.27 and higher correlation $(\rho=0.19)$ than the single front image. Applying the ResNet-50 pre-trained weights on the 3 combined image has lowest MAE in comparison with other models applied in the 3 combined images. Best MAE was observed using combined features of 3 images (applying 2D-CNN) and physical activity data (applying ConvLSTM) followed by transfer learning adopted from ResNet-50 replacing the 2D-CNN.

\subsubsection{Cox Proportional Hazard Model and Kaplan Meier Estimation}

Table 6.2 shows the results for the three approaches applying Cox proportional hazard model and log-rank test. 2D images (considering 3 images) and the combined features of back image and physical activity gives the highest hazard ratio of 1.14. ConvLSTM model applied on the physical activity data has the hazard ratio of 1.13 followed by the combined features (1.12) (front). However, for log-rank test 2D CNN applied on the 2D images (front) has the highest $\chi^{2}$-distances of 376.33 followed by the $2 \mathrm{D}$ images $(3$ images $)\left(\chi^{2}\right.$-distance $\left.=313.11\right)$. ResNet-50 applied on the 2D images (front, side, and back) also has the highest HR of 1.14 and $\chi^{2}$ distance of 305.63. While the combined features with ResNet-50 and ConvLSTM applied on the physical activity does not improve the results for both CoxPH model and logrank test. This clearly warrants further investigation, for instance, using other pre-trained weights for transfer learning. 


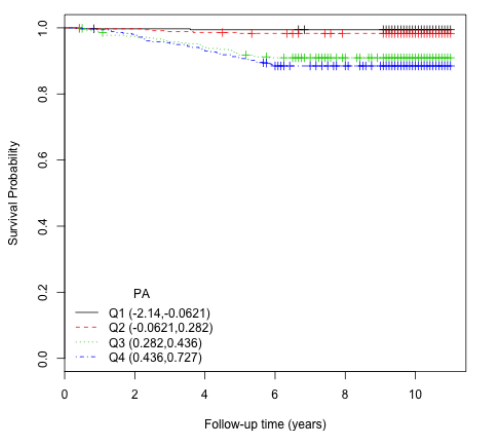

(a) Physical Activity

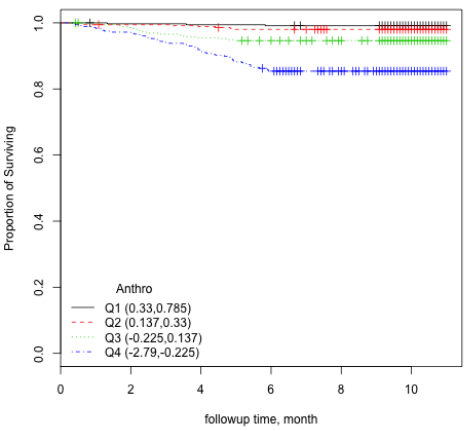

(b) 2D Images(front)

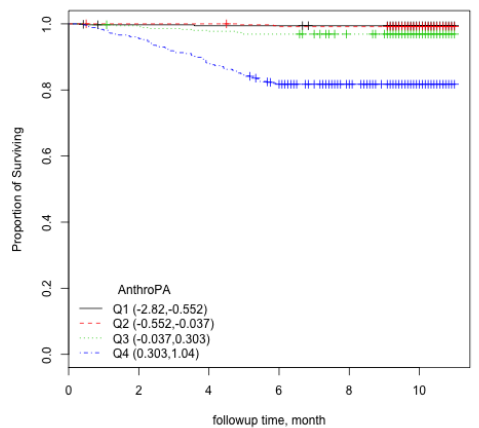

(c) Combined Features(3 Images)

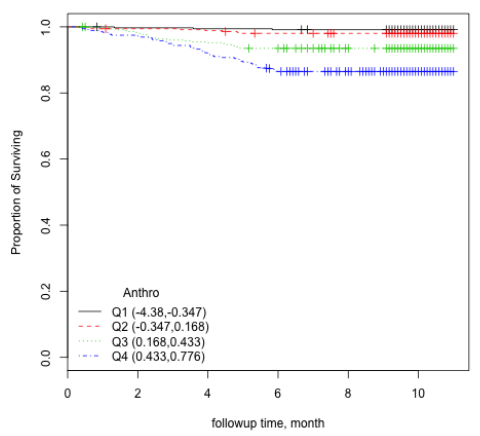

(d) 2D Images(3 Images)

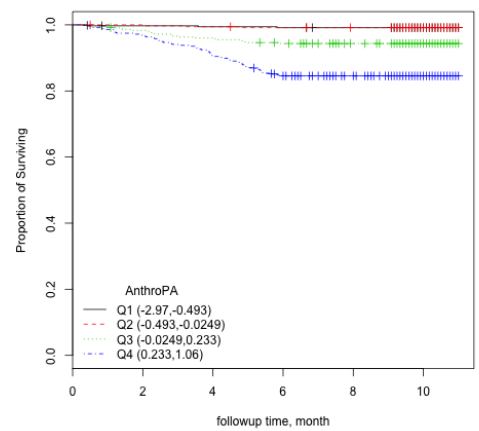

(e) Combined Features(front)

Figure 6.5: The Kaplan Meier curves for estimated biological ages (BA) based on the physical activity applying $\eta=\frac{C A-B A}{C A}$ for (a) ConvLSTM on the physical activity, (b) 2D CNN on the Makehuman images (front), (c) function architecture of 2D CNN, and ConvLSTM on the combined features (front), (d) 2D CNN on the Makehuman images (3 images), (e) function architecture of 2D CNN, and ConvLSTM on the combined features (3 images). Q1, Q2, Q3, and Q4 denote 1st, 2nd, 3rd, and 4th quartiles, respectively. 
Table 6.2: Results of the Cox proportional hazard (Cox PH) model and Log-rank test applied on the normalized biological age acceleration $\eta=(C A-B A) / C A$ for estimated biological ages.

\begin{tabular}{|l|c|c|c|c|}
\hline & \multicolumn{2}{|c|}{ CoxPH } & \multicolumn{3}{c|}{ Log Rank } \\
\hline & HR & p-value & Chi-Sq & p-value \\
\hline Physical Activity (ConvLSTM) & 1.13 & $1 \mathrm{E}-17$ & 177.68 & $1 \mathrm{E}-17$ \\
\hline 2D Images (2D CNN)(front) & 1.10 & $3 \mathrm{E}-20$ & $\mathbf{3 7 6 . 6 3}$ & $2 \mathrm{E}-22$ \\
\hline 2D Images (2D CNN)(side) & 1.09 & $4 \mathrm{E}-20$ & 353.91 & $4 \mathrm{E}-21$ \\
\hline 2D Images (2D CNN)(back) & 1.09 & $1 \mathrm{E}-19$ & 323.14 & $2 \mathrm{E}-10$ \\
\hline 2D Images (2D CNN)(front, side, back) & $\mathbf{1 . 1 4}$ & $2 \mathrm{E}-25$ & 313.11 & $1 \mathrm{E}-26$ \\
\hline Combined Features (2D-CNN, ConvLSTM)(front) & 1.12 & $1 \mathrm{E}-22$ & 213.93 & $2 \mathrm{E}-19$ \\
\hline Combined Features (2D-CNN, ConvLSTM)(side) & 1.13 & $7 \mathrm{E}-18$ & 203.41 & $8 \mathrm{E}-11$ \\
\hline Combined Features (2D-CNN, ConvLTM)(back) & $\mathbf{1 . 1 4}$ & $5 \mathrm{E}-18$ & 189.06 & $1 \mathrm{E}-17$ \\
\hline Combined Features (2D-CNN, ConvLSTM)(front, side, back) & 1.11 & $6 \mathrm{E}-13$ & 130.00 & $3 \mathrm{E}-23$ \\
\hline 2D Images (ResNet)(front, side, back) & $\mathbf{1 . 1 4}$ & $1 \mathrm{E}-26$ & 305.63 & $4 \mathrm{E}-18$ \\
\hline Combined Features (ResNet, ConvLSTM)(front, side, back) & 1.13 & $9 \mathrm{E}-18$ & 169.26 & $2 \mathrm{E}-17$ \\
\hline
\end{tabular}

\subsection{Discussion}

In this chapter, we have investigated different deep learning approaches on different types of data (e.g., locomotor physical activity and our plugin generated images from NHANES to Makehuman 3D models). To quantify how well the estimated biological age captures the health risk, we apply the Cox proportional hazard model and KM curves for analysis of all-cause mortality. The deep learning models such as convolutional LSTM, convolutional neural network were trained to exploit the dependence of the physiological/activity changes with age. In all cases, the deep learning approaches were trained to minimized the mean squared error (MSE) betweeen the chronological age and biological age. 


\section{Chapter 7}

\section{Future Work}

\subsection{Geometric deep learning}

Geometric deep learning is sort of an umbrella term for techniques that attempt to generalize

deep neural network to non-Euclidean domains. Recently, there is a growing interest to apply deep learning on non-Euclidean geometric data which arise in numerous applications. The generated 3D body shape data from MakeHuman is such an instance.

\subsection{Aging Biomarkers Discovery using Deep Learning}

One of the major challenges in human aging research is the lack of biomarkers that we can target and measure to track how anti-aging is affected by them. Typically, standard biomarkers are developed to measure a well-defined physiological process with particular clinical procedures depending on the use of predefined biomarkers. Hence, these may not be adapted for measuring the effects of aging. Therefore, there is a need to develop biomarkers which are objectively quantifiable and easily measurable characteristics of biological aging. Artificial intelligence offer alternatives for the aging biomarkers development than the tedious multi-stage process which requires proof of concept and experimental validation. 


\subsection{Biological Age from DNA methylation}

In a recent review paper [78], DNA methylation was identified as the most promising out of six most popular biological age estimators (telomere length, transcriptomic-based, proteomicbased, metabolomic- based, and composite biomarkers). Age has a deep connection with genome-wide DNA methylation levels. Millions of $\mathrm{CpG}$ dinucleotides in the human genome change with age. The DNA methylation array technique identifies the specific genome locations of the CpGs. Epigenetic age estimators are set of CpGs that are coupled with a mathematical algorithm to estimate the age of cells and tissues.

\subsection{Cardiorespiratory Fitness}

Cardiorespiratory fitness/endurance $(\mathrm{CRF})$ is the ability of a person's respiratory and circulatory systems to supply oxygen to skeletal muscles during sustained physical activity. CRF is correlated to numerous physiological systems, including cardiovascular, respiratory, and musculoskeletal systems [73]. Similar to biological age, CRF is also considered as one other reflection of whole-body health and function, and hence one of the predictors of all-cause mortality [73]. Locomotor physical activity dataset we used in this dissertation is also related to cardiorespiratory fitness. Thus, a potential future work will be to study the relationship between BA and CRF, for instance, using a deep learning framework.

\subsection{Variation of BA with ethnic group}

Ethnicity is expected to have influence on the performance of an age estimation scheme. Ethnicity included Caucasian (white), non-Hispanic black, Mexican American, other Hispanic, and others including multi-racial. We have observed performance for different ethnicity when using a single model for all. Table 7.1 shows the results for ethnic variations using the ConvLSTM model with the physical activity data applying Cox proportional hazard model 
and log-rank test. Non-Hispanic white subjects have the highest hazard ratio $(\mathrm{HR}=1.18)$ while the other Hispanic category has the lowest $(\mathrm{HR}=1.02)$. In term of log-rank test, non-Hispanic white has the highest $\chi^{2}$ distances and is significantly larger than other ethnic categories.

Table 7.1: Ethnic variation results of the Cox proportional hazard (Cox PH) model and Log-rank test.

\begin{tabular}{|l|c|c|c|l|}
\hline & \multicolumn{2}{|c|}{ CoxPH } & \multicolumn{2}{c|}{ Log Rank } \\
\hline & HR & p-value & Chi-Sq & p-value \\
\hline Mexican American & 1.16 & $3.13 \mathrm{E}-16$ & 55.99 & $4.22 \mathrm{E}-12$ \\
\hline Other Hispanic & 1.02 & $6.05 \mathrm{E}-01$ & 1.35 & $7.17 \mathrm{E}-01$ \\
\hline Non-Hispanic White & 1.18 & $1.10 \mathrm{E}-49$ & 251.76 & $0.00 \mathrm{E}+00$ \\
\hline Non-Hispanic Black & 1.15 & $2.94 \mathrm{E}-10$ & 35.24 & $1.09 \mathrm{E}-07$ \\
\hline Others & 1.09 & $1.11 \mathrm{E}-02$ & 9.57 & $2.26 \mathrm{E}-02$ \\
\hline
\end{tabular}




\section{Supplementary Materials}

\begin{tabular}{|c|c|c|c|c|c|c|c|c|}
\hline & \multicolumn{5}{|c|}{$\lambda=0$} & \multicolumn{4}{c|}{$\lambda=0.9$} \\
\hline $\mathbf{N}$ & \multicolumn{2}{|c|}{ SVM } & \multicolumn{2}{|c|}{$\mathbf{R F}$} & \multicolumn{2}{|c|}{ SVM } & \multicolumn{2}{|c|}{$\mathbf{R F}$} \\
\hline & mae & correlation & mae & correlation & mae & correlation & mae & correlation \\
\hline 1 & 13.73 & 0.51 & 14.29 & 0.52 & 13.72 & 0.51 & 14.26 & 0.53 \\
\hline 2 & 13.7 & 0.51 & 14.19 & 0.51 & 13.95 & 0.5 & 13.9 & 0.54 \\
\hline 4 & 13.67 & 0.52 & 14.03 & 0.53 & 14.19 & 0.5 & 13.95 & 0.55 \\
\hline 8 & 13.59 & 0.52 & 13.93 & 0.54 & 13.99 & 0.51 & 13.5 & 0.58 \\
\hline 10 & 13.58 & 0.52 & 13.94 & 0.54 & 13.96 & 0.51 & 13.42 & 0.59 \\
\hline 16 & 13.56 & 0.53 & 13.98 & 0.54 & 13.89 & 0.52 & 13.25 & 0.59 \\
\hline 20 & 13.53 & 0.53 & 13.73 & 0.56 & 13.83 & 0.52 & 13.29 & 0.59 \\
\hline 25 & 13.54 & 0.53 & 13.7 & 0.56 & 13.77 & 0.53 & 13.12 & 0.6 \\
\hline 30 & 13.56 & 0.53 & 13.53 & 0.57 & 13.77 & 0.53 & 13.12 & 0.6 \\
\hline 35 & 13.58 & 0.53 & 13.66 & 0.56 & 13.72 & 0.53 & 12.86 & 0.62 \\
\hline 40 & 13.6 & 0.53 & 13.56 & 0.57 & 13.69 & 0.53 & 12.86 & 0.62 \\
\hline
\end{tabular}

Table S1: Variations of window size for computing the moving averages after log-transformation.

\begin{tabular}{|c|c|c|c|c|}
\hline \multirow{2}{*}{$\lambda$} & \multicolumn{2}{|c|}{ SVM } & \multicolumn{2}{c|}{ RF } \\
\cline { 2 - 5 } & mae & correlation & mae & correlation \\
\hline 0.2 & 13.50 & 0.53 & 13.42 & 0.58 \\
\hline 0.3 & 13.45 & 0.54 & 13.42 & 0.58 \\
\hline 0.4 & 13.46 & 0.54 & 13.33 & 0.59 \\
\hline 0.5 & 13.48 & 0.54 & 13.23 & 0.6 \\
\hline 0.65 & 13.53 & 0.54 & 13.03 & 0.61 \\
\hline 0.75 & 13.57 & 0.54 & 12.99 & 0.61 \\
\hline 0.85 & 13.65 & 0.53 & 13.01 & 0.61 \\
\hline 0.9 & 13.72 & 0.53 & 12.86 & 0.62 \\
\hline 0.95 & 13.74 & 0.53 & 12.98 & 0.61 \\
\hline 1.1 & 13.86 & 0.53 & 12.87 & 0.61 \\
\hline
\end{tabular}

Table S2: Choosing $\lambda$ parameters for the Box-Cox transformation. 


\section{Bibliography}

[1] Cyberware scanner @ONLINE.

[2] National Health and Nutrition Examination Survey (NHANES) Anthropometry Procedures Manual. Technical report, National Center for Health Statistics @ONLINE, December 2002.

[3] Nhanes (2002) national health and nutrition examination survey anthropometry procedures manual. technical report, national center for health statistics @ONLINE, 2002.

[4] Nchs (2006) analytic and reporting guidelines: The national health and nutrition examination survey (nhanes). technical report, national center for health statistics, centers for disease control and prevention, hyattsville, maryland. @ONLINE, 2006.

[5] Ieee conference on decision and control and european control conference @ONLINE, 2011.

[6] Nchs (2011) national health and nutrition examination survey data. @ONLINE, 2011.

[7] Us centers for disease control (CDC), recommended immunization schedule for adults aged 19 years or older, by vaccine and age group, united states @ONLINE, December 2017.

[8] International Society for Physical Activity and Health. @ONLINE, September 2018. 
[9] National Health and Nutrition Examination Survey (NHANES) Anthropometry Procedures Manual. Technical report, National Center for Health Statistics @ONLINE, December 2018.

[10] Neural networks for machine learning. @ONLINE, November 2018.

[11] WHO. Global Physical Activity Surveillance. @ONLINE, September 2018.

[12] Donald Adjeroh, Deng Cao, Marco Piccirilli, and Arun Ross. Predictability and correlation in human metrology. In Information Forensics and Security (WIFS), 2010 IEEE International Workshop on, pages 1-6. IEEE, 2010.

[13] Thomas R Alley. Social and Applied Aspects of Perceiving Faces. Psychology Press, 2013.

[14] David B Allison, Myles S Faith, Moonseong Heo, and Donald P Kotler. Hypothesis concerning the u-shaped relation between body mass index and mortality. American Journal of Epidemiology, 146(4):339-349, 1997.

[15] David B Allison, SK Zhu, Michael Plankey, Myles S Faith, and Moonseong Heo. Differential associations of body mass index and adiposity with all-cause mortality among men in the first and second national health and nutrition examination surveys (nhanes i and nhanes ii) follow-up studies. International Journal of Obesity and Related Metabolic Disorders: Journal of The International Association for the Study of Obesity, 26(3):410-416, 2002.

[16] André Altmann, Laura Toloşi, Oliver Sander, and Thomas Lengauer. Permutation importance: A corrected feature importance measure. Bioinformatics, 26(10):1340$1347,2010$.

[17] Kaarin J Anstey, Stephen R Lord, and Glen A Smith. Measuring human functional age: A review of empirical findings. Experimental Aging Research, 22(3):245-266, 1996. 
[18] M Ashwell, P Gunn, and S Gibson. Waist-to-height ratio is a better screening tool than waist circumference and bmi for adult cardiometabolic risk factors: systematic review and meta-analysis. Obesity Reviews, 13(3):275-286, 2012.

[19] Margarett Ashwell, Tim J Cole, and Adrian K Dixon. Obesity: new insight into the anthropometric classification of fat distribution shown by computed tomography. $B M J$, 290(6483):1692-1694, 1985.

[20] Manuel Bastioni, Simone Re, and Shakti Misra. Ideas and methods for modeling 3d human figures: The principal algorithms used by makehuman and their implementation in a new approach to parametric modeling. In Proceedings of the 1st Bangalore Annual Compute Conference, page 10. ACM, 2008.

[21] Daniel W Belsky, Avshalom Caspi, Renate Houts, Harvey J Cohen, David L Corcoran, Andrea Danese, HonaLee Harrington, Salomon Israel, Morgan E Levine, Jonathan D Schaefer, et al. Quantification of biological aging in young adults. Proceedings of the National Academy of Sciences, 112(30):E4104-E4110, 2015.

[22] Janne Bigaard, Kirsten Frederiksen, Anne Tjønneland, Birthe Lykke Thomsen, Kim Overvad, Berit Lillienthal Heitmann, and Thorkild IA Sørensen. Body fat and fat-free mass and all-cause mortality. Obesity Research, 12(7):1042-1049, 2004.

[23] Janne Bigaard, Anne Tjønneland, Birthe Lykke Thomsen, Kim Overvad, Berit Lilienthal Heitmann, and Thorkild IA Sørensen. Waist circumference, bmi, smoking, and mortality in middle-aged men and women. Obesity Research, 11(7):895-903, 2003.

[24] Sven Bocklandt, Wen Lin, Mary E Sehl, Francisco J Sánchez, Janet S Sinsheimer, Steve Horvath, and Eric Vilain. Epigenetic predictor of age. PLoS ONE, 6(6):e14821, 2011.

[25] George EP Box and David R Cox. An analysis of transformations. Journal of the Royal Statistical Society. Series B (Methodological), pages 211-252, 1964. 
[26] Lucy M Browning, Shiun Dong Hsieh, and Margaret Ashwell. A systematic review of waist-to-height ratio as a screening tool for the prediction of cardiovascular disease and diabetes: 0.5 could be a suitable global boundary value. Nutrition Research Reviews, 23(02):247-269, 2010.

[27] Eugenia E Calle, Carmen Rodriguez, Kimberly Walker-Thurmond, and Michael J Thun. Overweight, obesity, and mortality from cancer in a prospectively studied cohort of us adults. New England Journal of Medicine, 348(17):1625-1638, 2003.

[28] J Campisi. The biology of replicative senescence. European Journal of Cancer, 33(5):703-709, 1997.

[29] Judith Campisi. Cellular senescence and cell death. Physiological Basis of Aging and Geriatrics, pages 47-59, 2003.

[30] Deng Cao, Cunjian Chen, Donald Adjeroh, and Arun Ross. Predicting gender and weight from human metrology using a copula model. In Biometrics: Theory, Applications and Systems (BTAS), 2012 IEEE Fifth International Conference on, pages 162-169. IEEE, 2012.

[31] Mercedes R Carnethon, Peter John D De Chavez, Mary L Biggs, Cora E Lewis, James S Pankow, Alain G Bertoni, Sherita H Golden, Kiang Liu, Kenneth J Mukamal, Brenda Campbell-Jenkins, et al. Association of weight status with mortality in adults with incident diabetes. JAMA, 308(6):581-590, 2012.

[32] CJ Caspersen and L DiPietro. National estimates of physical activity among older adults. Medicine and Science in Sports and Exercise, 23(4 supplement):106, 1991.

[33] E Cevenini, L Invidia, Francesco Lescai, S Salvioli, P Tieri, G Castellani, and C Franceschi. Human models of aging and longevity. Expert opinion on biological therapy, 8(9):1393-1405, 2008. 
[34] June M Chan, Eric B Rimm, Graham A Colditz, Meir J Stampfer, and Walter C Willett. Obesity, fat distribution, and weight gain as risk factors for clinical diabetes in men. Diabetes Care, 17(9):961-969, 1994.

[35] Il Haeng Cho, Kyung S Park, and Chang Joo Lim. An empirical comparative study on biological age estimation algorithms with an application of Work Ability Index (WAI). Mechanisms of Ageing and Development, 131(2):69-78, 2010.

[36] Caroline Chua, Joseph A Cote, and Siew Meng Leong. The antecedents of cognitive age. NA-Advances in Consumer Research Volume 17, 1990.

[37] Graham A Colditz, Walter C Willett, Andrea Rotnitzky, and JoAnn E Manson. Weight gain as a risk factor for clinical diabetes mellitus in women. Annals of Internal Medicine, 122(7):481-486, 1995.

[38] JH Cole, SJ Ritchie, ME Bastin, Hernández MC Valdés, S Muñoz Maniega, N Royle, J Corley, A Pattie, SE Harris, Q Zhang, et al. Brain age predicts mortality. Molecular Psychiatry, 2017.

[39] David Roxbee Cox and David Oakes. Analysis of Survival Data, volume 21. CRC Press, 1984.

[40] Nathan Daniell, Timothy Olds, and Grant Tomkinson. Technical note: Criterion validity of whole body surface area equations: A comparison using 3d laser scanning. American Journal of Physical Anthropology, 148(1):148-155, 2012.

[41] David L Davies and Donald W Bouldin. A cluster separation measure. IEEE Transactions on Pattern Analysis and Machine Intelligence, (2):224-227, 1979.

[42] L DiPietro, DF Williamson, CJ Caspersen, and E Eaker. The descriptive epidemiology of selected physical activities and body weight among adults trying to lose weight: the behavioral risk factor surveillance system survey, 1989. International Journal of 
Obesity and Related Metabolic Disorders: Journal of the International Association for the Study of Obesity, 17(2):69-76, 1993.

[43] Loretta DiPietro. Physical activity in aging: changes in patterns and their relationship to health and function. The Journals of Gerontology Series A: Biological Sciences and Medical Sciences, 56(suppl_2):13-22, 2001.

[44] Wolfram Doehner, Andrew Clark, and Stefan D Anker. The obesity paradox: weighing the benefit. European Heart Journal, page ehp339, 2009.

[45] Timothy Dozat. Incorporating nesterov momentum into adam. ICLR Workshop, 2016.

[46] Joseph C Dunn. A fuzzy relative of the ISODATA process and its use in detecting compact well-separated clusters. 1973.

[47] Robert H Eckel, Ronald M Krauss, et al. American heart association call to action: Obesity as a major risk factor for coronary heart disease. Circulation, 97(21):20992100, 1998.

[48] Monika Eipel, Felix Mayer, Tanja Arent, Marcelo RP Ferreira, Carina Birkhofer, Uwe Gerstenmaier, Ivan G Costa, Stefanie Ritz-Timme, and Wolfgang Wagner. Epigenetic age predictions based on buccal swabs are more precise in combination with cell typespecific DNA methylation signatures. Aging, 8(5), 2016.

[49] Krista Fischer, Johannes Kettunen, Peter Würtz, Toomas Haller, Aki S Havulinna, Antti J Kangas, Pasi Soininen, Tõnu Esko, Mari-Liis Tammesoo, Reedik Mägi, et al. Biomarker profiling by nuclear magnetic resonance spectroscopy for the prediction of all-cause mortality: An observational study of 17,345 persons. PLoS Med, 11(2):e1001606, 2014.

[50] Katherine M Flegal, Margaret D Carroll, Cynthia L Ogden, and Lester R Curtin. Prevalence and trends in obesity among us adults, 1999-2008. JAMA, 303(3):235-241, 2010. 
[51] Katherine M Flegal, Barry I Graubard, David F Williamson, and Mitchell H Gail. Excess deaths associated with underweight, overweight, and obesity. JAMA, 293(15):1861-1867, 2005.

[52] Katherine M Flegal, Brian K Kit, Heather Orpana, and Barry I Graubard. Association of all-cause mortality with overweight and obesity using standard body mass index categories: a systematic review and meta-analysis. JAMA, 309(1):71-82, 2013.

[53] Yun Fu, Guodong Guo, and Thomas S Huang. Age synthesis and estimation via faces: A survey. IEEE Transactions on Pattern Analysis and Machine Intelligence, 32(11):1955-1976, 2010.

[54] Yun Fu and Thomas S Huang. Human age estimation with regression on discriminative aging manifold. IEEE Transactions on Multimedia, 10(4):578-584, 2008.

[55] Lisa Gallicchio, Meghan A McSorley, Craig J Newschaffer, Han-Yao Huang, Lucy W Thuita, Sandra C Hoffman, and Kathy J Helzlsouer. Body mass, polymorphisms in obesity-related genes, and the risk of developing breast cancer among women with benign breast disease. Cancer Detection and Prevention, 31(2):95-101, 2007.

[56] Xin Geng, Chao Yin, and Zhi-Hua Zhou. Facial age estimation by learning from label distributions. IEEE Transactions on Pattern Analysis and Machine Intelligence, 35(10):2401-2412, 2013.

[57] Xin Geng, Zhi-Hua Zhou, and Kate Smith-Miles. Automatic age estimation based on facial aging patterns. IEEE Transactions on Pattern Analysis and Machine Intelligence, 29(12):2234-2240, 2007.

[58] Xavier Glorot and Yoshua Bengio. Understanding the difficulty of training deep feedforward neural networks. In Proceedings of the Thirteenth International Conference on Artificial Intelligence and Statistics, pages 249-256, 2010. 
[59] Guodong Guo, Yun Fu, Thomas S Huang, and Charles R Dyer. Locally adjusted robust regression for human age estimation. IEEE Workshop on Applications of Computer Vision, 51:61801, 2008.

[60] Guodong Guo and Guowang Mu. Simultaneous dimensionality reduction and human age estimation via kernel partial least squares regression. In Computer Vision and Pattern Recognition (CVPR), 2011 IEEE Conference on, pages 657-664. IEEE, 2011.

[61] Guodong Guo, Guowang Mu, Yun Fu, Charles R Dyer, and Thomas S Huang. A study on automatic age estimation using a large database. In ICCV, pages 1986-1991, 2009.

[62] Guodong Guo, Guowang Mu, Yun Fu, and Thomas S Huang. Human age estimation using bio-inspired features. In Computer Vision and Pattern Recognition, 2009. CVPR 2009. IEEE Conference on, pages 112-119. IEEE, 2009.

[63] Regina Guthold, Gretchen A Stevens, Leanne M Riley, and Fiona C Bull. Worldwide trends in insufficient physical activity from 2001 to 2016: a pooled analysis of 358 population-based surveys with 1. 9 million participants. The Lancet Global Health, 2018.

[64] Yuya Hamano, Sho Manabe, Chie Morimoto, Shuntaro Fujimoto, and Keiji Tamaki. Forensic age prediction for saliva samples using methylation-sensitive high resolution melting: exploratory application for cigarette butts. Scientific Reports, 7(1):10444, 2017.

[65] Hu Han, Charles Otto, Xiaoming Liu, and Anil K Jain. Demographic estimation from face images: Human vs. machine performance. IEEE Transactions on Pattern Analysis and Machine Intelligence, 37(6):1148-1161, 2015.

[66] TS Han, G McNeill, JC Seidell, and MEJ Lean. Predicting intra-abdominal fatness from anthropometric measures: the influence of stature. International Journal of Obesity, 21(7):587-593, 1997. 
[67] Denham Harman. The free radical theory of aging. Antioxidants and Redox Signaling, 5(5):557-561, 2003.

[68] Sen He and Xiaoping Chen. Could the new body shape index predict the new onset of diabetes mellitus in the chinese population? PloS One, 8(1):e50573, 2013.

[69] Sepp Hochreiter and Jürgen Schmidhuber. Long short-term memory. Neural computation, 9(8):1735-1780, 1997.

[70] Steve Horvath. DNA methylation age of human tissues and cell types. Genome Biology, 14(10):3156, 2013.

[71] James S House, James M Lepkowski, Ann M Kinney, Richard P Mero, Ronald C Kessler, and A Regula Herzog. The social stratification of aging and health. Journal of Health and Social Behavior, pages 213-234, 1994.

[72] SD Hsieh, H Yoshinaga, and T Muto. Waist-to-height ratio, a simple and practical index for assessing central fat distribution and metabolic risk in japanese men and women. International journal of obesity, 27(5):610-616, 2003.

[73] Mary T Imboden, Matthew P Harber, Mitchell H Whaley, W Holmes Finch, Derron L Bishop, and Leonard A Kaminsky. Cardiorespiratory fitness and mortality in healthy men and women. Journal of the American College of Cardiology, 72(19):2283-2292, 2018.

[74] Stephen HD Jackson, Martin R Weale, and Robert A Weale. Biological age - what is it and can it be measured? Archives of Gerontology and Geriatrics, 36(2):103-115, 2003.

[75] Ian Janssen, Steven B Heymsfield, David B Allison, Donald P Kotler, and Robert Ross. Body mass index and waist circumference independently contribute to the prediction of nonabdominal, abdominal subcutaneous, and visceral fat. The American journal of clinical nutrition, 75(4):683-688, 2002. 
[76] Ian Janssen and Amy E Mark. Elevated body mass index and mortality risk in the elderly. Obesity Reviews, 8(1):41-59, 2007.

[77] Ian Jolliffe. Principal Component Analysis. Wiley Online Library, 2002.

[78] Juulia Jylhävä, Nancy L Pedersen, and Sara Hägg. Biological age predictors. EBioMedicine, 21:29-36, 2017.

[79] Alka M Kanaya, Eric Vittinghoff, Michael G Shlipak, Helaine E Resnick, Marjolein Visser, Deborah Grady, and Elizabeth Barrett-Connor. Association of total and central obesity with mortality in postmenopausal women with coronary heart disease. American journal of epidemiology, 158(12):1161-1170, 2003.

[80] Edward L Kaplan and Paul Meier. Nonparametric estimation from incomplete observations. Journal of the American Statistical Association, 53(282):457-481, 1958.

[81] Satish Kenchaiah, Jane C Evans, Daniel Levy, Peter WF Wilson, Emelia J Benjamin, Martin G Larson, William B Kannel, and Ramachandran S Vasan. Obesity and the risk of heart failure. New England Journal of Medicine, 347(5):305-313, 2002.

[82] Diederik P Kingma and Jimmy Ba. Adam: A method for stochastic optimization. arXiv preprint arXiv:1412.6980, 2014.

[83] Petr Klemera and Stanislav Doubal. A new approach to the concept and computation of biological age. Mechanisms of Ageing and Development, 127(3):240-248, 2006.

[84] Edward L Kom, Barry I Graubard, and Douglas Midthune. Time-to-event analysis of longitudinal follow-up of a survey: choice of the time-scale. American Journal of Epidemiology, 145(1):72-80, 1997.

[85] Nir Y Krakauer and Jesse C Krakauer. A new body shape index predicts mortality hazard independently of Body Mass Index. PLoS ONE, 7(7):e39504, 2012. 
[86] Heikki Kröger, Antero Kotaniemi, Liisa Kröger, and Esko Alhava. Development of bone mass and bone density of the spine and femoral neck - a prospective study of 65 children and adolescents. Bone and Mineral, 23(3):171-182, 1993.

[87] J Krøll and O Saxtrup. On the use of regression analysis for the estimation of human biological age. Biogerontology, 1(4):363-368, 2000.

[88] Mitja Lainscak, Stephan von Haehling, Wolfram Doehner, and Stefan D Anker. The obesity paradox in chronic disease: facts and numbers. Journal of Cachexia, Sarcopenia and Muscle, 3(1):1-4, 2012.

[89] Morgan E Levine. Modeling the rate of senescence: can estimated biological age predict mortality more accurately than chronological age? Journals of Gerontology Series A: Biomedical Sciences and Medical Sciences, 68(6):667-674, 2012.

[90] Edward A Lew and Lawrence Garfinkel. Variations in mortality by weight among 750,000 men and women. Journal of Chronic Diseases, 32(8):563-576, 1979.

[91] Wen-Cheng Li, I-Chuan Chen, Yu-Che Chang, Song-Seng Loke, Shih-Hao Wang, and Kuang-Yu Hsiao. Waist-to-height ratio, waist circumference, and body mass index as indices of cardiometabolic risk among 36,642 taiwanese adults. European Journal of Nutrition, 52(1):57-65, 2013.

[92] Jiwen Lu and Yap-Peng Tan. Gait-based human age estimation. IEEE Transactions on Information Forensics and Security, 5(4):761-770, 2010.

[93] M Maber, HM Liversidge, and MP Hector. Accuracy of age estimation of radiographic methods using developing teeth. Forensic Science International, 159:S68-S73, 2006.

[94] George L Maddox and Elizabeth B Douglass. Self-assessment of health: A longitudinal study of elderly subjects. Journal of Health and Social Behavior, pages 87-93, 1973. 
[95] JoAnn E Manson, Walter C Willett, Meir J Stampfer, Graham A Colditz, David J Hunter, Susan E Hankinson, Charles H Hennekens, and Frank E Speizer. Body weight and mortality among women. New England Journal of Medicine, 333(11):677-685, 1995.

[96] Daniel L McGee, Diverse Populations Collaboration, et al. Body mass index and mortality: a meta-analysis based on person-level data from twenty-six observational studies. Annals of Epidemiology, 15(2):87-97, 2005.

[97] Arnold Mitnitski, Susan E Howlett, and Kenneth Rockwood. Heterogeneity of human aging and its assessment. Journals of Gerontology Series A: Biomedical Sciences and Medical Sciences, 72(7):877-884, 2016.

[98] Ali H Mokdad, Earl S Ford, Barbara A Bowman, William H Dietz, Frank Vinicor, Virginia S Bales, and James S Marks. Prevalence of obesity, diabetes, and obesityrelated health risk factors, 2001. JAMA, 289(1):76-79, 2003.

[99] Bjørn Mørkedal, Pål R Romundstad, and Lars J Vatten. Informativeness of indices of blood pressure, obesity and serum lipids in relation to ischaemic heart disease mortality: the hunt-ii study. European Journal of Epidemiology, 26(6):457-461, 2011.

[100] Arsalan Manzoor Mughal, Nuzhat Hassan, and Anwar Ahmed. Bone age assessment methods: A critical review. Pakistan journal of medical sciences, 30(1):211, 2014.

[101] Kevin P Murphy. Machine learning: A probabilistic perspective. MIT press, 2012.

[102] Bingbing Ni, Zheng Song, and Shuicheng Yan. Web image mining towards universal age estimator. In Proceedings of the 17th ACM International Conference on Multimedia, pages 85-94. ACM, 2009.

[103] Jari-Erik Nurmi. Age, sex, social class, and quality of family interaction as determinants of adolescents' future orientation: A developmental task interpretation. Adolescence, $22(88): 977,1987$. 
[104] Cynthia L Ogden, Margaret D Carroll, Brian K Kit, and Katherine M Flegal. Prevalence of obesity and trends in body mass index among us children and adolescents, 1999-2010. JAMA, 307(5):483-490, 2012.

[105] Cynthia L Ogden, National Center for Health Statistics (US), et al. Prevalence of obesity in the united states, 2009-2010, 2012.

[106] M Ohrvall, L Berglund, and B Vessby. Sagittal abdominal diameter compared with other anthropometric measurements in relation to cardiovascular risk. International Journal of Obesity and Related Metabolic Disorders: Journal of the International Association for the Study of Obesity, 24(4):497-501, 2000.

[107] Altan Onat, Gülay Hergenç, Hüsniye Yüksel, Günay Can, Erkan Ayhan, Zekeriya Kaya, and Dursun Dursunoğlu. Neck circumference as a measure of central obesity: associations with metabolic syndrome and obstructive sleep apnea syndrome beyond waist circumference. Clinical Nutrition, 28(1):46-51, 2009.

[108] Raj S Padwal, Nicholas M Pajewski, David B Allison, and Arya M Sharma. Using the edmonton obesity staging system to predict mortality in a population-representative cohort of people with overweight and obesity. Canadian Medical Association Journal, 183(14):E1059-E1066, 2011.

[109] Halfdan Petursson, Johann A Sigurdsson, Calle Bengtsson, Tom IL Nilsen, and Linn Getz. Body configuration as a predictor of mortality: comparison of five anthropometric measures in a 12 year follow-up of the norwegian hunt 2 study. PloS One, 6(10):e26621, 2011.

[110] Marco Piccirilli. Machine Learning Approaches to Human Body Shape Analysis. PhD thesis, West Virginia University, 2018.

[111] Marco Piccirilli, Gianfranco Doretto, and Donald Adjeroh. A framework for analyzing the whole body surface area from a single view. PloS one, 12(1):e0166749, 2017. 
[112] Marie-Christine Pouliot, Jean-Pierre Després, Simone Lemieux, Sital Moorjani, Claude Bouchard, Angelo Tremblay, André Nadeau, and Paul J Lupien. Waist circumference and abdominal sagittal diameter: best simple anthropometric indexes of abdominal visceral adipose tissue accumulation and related cardiovascular risk in men and women. The American Journal of Cardiology, 73(7):460-468, 1994.

[113] Richie Poulton, Terrie E Moffitt, and Phil A Silva. The Dunedin multidisciplinary health and development study: Overview of the first 40 years, with an eye to the future. Social Psychiatry and Psychiatric Epidemiology, 50(5):679-693, 2015.

[114] Evgeny Putin, Polina Mamoshina, Alexander Aliper, Mikhail Korzinkin, Alexey Moskalev, Alexey Kolosov, Alexander Ostrovskiy, Charles Cantor, Jan Vijg, and Alex Zhavoronkov. Deep biomarkers of human aging: Application of deep neural networks to biomarker development. Aging, 8(5):1-021, 2016.

[115] Timothy V Pyrkov, Konstantin Slipensky, Mikhail Barg, Alexey Kondrashin, Boris Zhurov, Alexander Zenin, Mikhail Pyatnitskiy, Leonid Menshikov, Sergei Markov, and Peter O Fedichev. Extracting biological age from biomedical data via deep learning: too much of a good thing? Scientific Reports, 8(1):5210, 2018.

[116] Susan B Racette, Susan S Deusinger, and Robert H Deusinger. Obesity: Overview of prevalence, etiology, and treatment. Physical Therapy, 83(3):276-288, 2003.

[117] Geetha Raghuveer. Lifetime cardiovascular risk of childhood obesity. The American Journal of Clinical Nutrition, 91(5):1514S-1519S, 2010.

[118] Syed Ashiqur Rahman and Donald Adjeroh. Surface-Based Body Shape Index and Its Relationship with All-Cause Mortality. PLoS ONE, 10(12):e0144639, 2015.

[119] Narayanan Ramanathan and Rama Chellappa. Modeling age progression in young faces. In 2006 IEEE Computer Society Conference on Computer Vision and Pattern Recognition (CVPR'06), volume 1, pages 387-394. IEEE, 2006. 
[120] Christoph Richter, Jeen-Woo Park, and Bruce N Ames. Normal oxidative damage to mitochondrial and nuclear DNA is extensive. Proceedings of the National Academy of Sciences, 85(17):6465-6467, 1988.

[121] S Ritz-Timme, C Cattaneo, MJ Collins, ER Waite, HW Schütz, H-J Kaatsch, and HIM Borrman. Age estimation: The state of the art in relation to the specific demands of forensic practise. International Journal of Legal Medicine, 113(3):129-136, 2000.

[122] Kathleen M Robinette, Hans Daanen, and Eric Paquet. The caesar project: A 3-D surface anthropometry survey. In 3-D Digital Imaging and Modeling, 1999. Proceedings. Second International Conference on, pages 380-386. IEEE, 1999.

[123] Abel Romero-Corral, Virend Somers, Francisco Lopez-Jimenez, Yoel Korenfeld, Suzanne Palin, Kristien Boclaert, Simona Boarin, Justo Sierra-Johnson, and Asad Rahirn. 3-d body scanners, body volume index: A novel, reproducible and automated anthropometric tool associated with cardiometabolic biomarkers. In Obesity, volume 16, pages S124-S124. Obesity. vol. 16. Nature Publishing Group 75 Varick st, 9th flr, New York, NY 10013-1917 USA, 2008.

[124] Warren C Sanderson and Sergei Scherbov. Measuring the speed of aging across population subgroups. PLoS ONE, 9(5):e96289, 2014.

[125] A Schmeling, C Grundmann, A Fuhrmann, H-J Kaatsch, B Knell, F Ramsthaler, W Reisinger, T Riepert, S Ritz-Timme, FW Rösing, et al. Criteria for age estimation in living individuals. International Journal of Legal Medicine, 122(6):457-460, 2008.

[126] Harald J Schneider, Nele Friedrich, Jens Klotsche, Lars Pieper, Matthias Nauck, Ulrich John, Marcus Dorr, Stephan Felix, Hendrik Lehnert, David Pittrow, et al. The predictive value of different measures of obesity for incident cardiovascular events and mortality. The Journal of Clinical Endocrinology \& Metabolism, 95(4):1777-1785, 2010. 
[127] Paola Sebastiani, Bharat Thyagarajan, Fangui Sun, Nicole Schupf, Anne B Newman, Monty Montano, and Thomas T Perls. Biomarker signatures of aging. Aging Cell, 16(2):329-338, 2017.

[128] Lior Shamir. Automatic age estimation by hand photos. Computer Science Letters, $3(1), 2011$.

[129] BRIAN J Sharkey. Functional vs chronologic age. Medicine and Science in Sports and Exercise, 19(2):174-178, 1987.

[130] Borys Shuter and Alireza Aslani. Body surface area: Du bois and du bois revisited. European Journal of Applied Physiology, 82(3):250-254, 2000.

[131] MB Snijder, RM Van Dam, M Visser, and JC Seidell. What aspects of body fat are particularly hazardous and how do we measure them? International Journal of Epidemiology, 35(1):83-92, 2006.

[132] Xiao Song and Xiao-Hua Zhou. A semiparametric approach for the covariate specific roc curve with survival outcome. Statistica Sinica, pages 947-965, 2008.

[133] Urszula Stańczyk and Lakhmi C Jain. Feature selection for data and pattern recognition. Springer, 2015.

[134] Thomas Stephens and Carl J Caspersen. The demography of physical activity. Physical Activity, Fitness, and Health: International Proceedings and Consensus Statement, pages 204-213, 1994.

[135] Abd Tahrani, Kristien Boelaert, Richard Barnes, Suzanne Palin, Annmarie Field, Helen Redmayne, Lisa Aytok, and Asad Rahim. Body volume index: Time to replace body mass index? 2008.

[136] Robert Tibshirani. Regression shrinkage and selection via the lasso. Journal of the Royal Statistical Society. Series B (Methodological), pages 267-288, 1996. 
[137] Matteo Tosato, Valentina Zamboni, Alessandro Ferrini, and Matteo Cesari. The aging process and potential interventions to extend life expectancy. Clinical Interventions in Aging, 2(3):401, 2007.

[138] Philip Treleaven and JCK Wells. 3d body scanning and healthcare applications. Computer, 40(7):28-34, 2007.

[139] Xiaolong Wang, Rui Guo, and Chandra Kambhamettu. Deeply-learned feature for age estimation. In 2015 IEEE Winter Conference on Applications of Computer Vision, pages 534-541. IEEE, 2015.

[140] Jonathan CK Wells, Philip Treleaven, and Tim J Cole. Bmi compared with 3dimensional body shape: the uk national sizing survey. The American Journal of Clinical Nutrition, 85(2):419-425, 2007.

[141] WHO. Obesity: preventing and managing the global epidemic. Number 894. World Health Organization, 2000.

[142] Guy Williams. A review of the most commonly used dental age estimation techniques. J Forensic Odontostomatol, 19:9-17, 2001.

[143] Baiqiang Xia, Boulbaba Ben Amor, Mohamed Daoudi, and Hassen Drira. Can 3D shape of the face reveal your age? In International Conference on Computer Vision Theory and Applications (VISAPP), volume 2, pages 5-13. IEEE, 2014.

[144] SHI Xingjian, Zhourong Chen, Hao Wang, Dit-Yan Yeung, Wai-Kin Wong, and Wangchun Woo. Convolutional lstm network: A machine learning approach for precipitation nowcasting. In Advances in neural information processing systems, pages 802-810, 2015.

[145] Cheng Xu, Hongzhu Qu, Guangyu Wang, Bingbing Xie, Yi Shi, Yaran Yang, Zhao Zhao, Lan Hu, Xiangdong Fang, Jiangwei Yan, et al. A novel strategy for forensic 
age prediction by DNA methylation and support vector regression model. Scientific Reports, 5, 2015.

[146] Chi-Yuang Yu, Ching-Hua Lin, and Yi-Hsueh Yang. Human body surface area database and estimation formula. Burns, 36(5):616-629, 2010.

[147] A Zerilli, E Baccino, H Lamendin, JC Tavernier, JF Humbert, and RM Nossintchouk. A simple technique for age estimation in adult corpses: The two criteria dental method. Journal of Forensic Science, 37(5):1373-1379, 1992.

[148] Zhe-Qing Zhang, Juan Deng, Li-Ping He, Wen-Hua Ling, Yi-Xiang Su, and Yu-Ming Chen. Comparison of various anthropometric and body fat indices in identifying cardiometabolic disturbances in chinese men and women. PloS one, 8(8):e70893, 2013. 